Der Medizinischen Fakultät der Georg-August-Universität Göttingen eingereicht von Prof. Dr. med. B. Mollenhauer

\title{
DeNoPa Kassel: Die prospektive Langzeit-Follow-up-Studie zu Biomarkern und nicht-motorischen Symptomen bei Morbus Parkinson
}

\section{Pilotstudie baseline}

\author{
INAUGURAL-DISSERTATION \\ zur Erlangung des Doktorgrades \\ der Medizinischen Fakultät \\ der Georg-August-Universität zu Göttingen
}

vorgelegt von

STEFANIE WERNER

aus

Leverkusen

Göttingen 2012 
Diese Arbeit wurde durchgeführt in der Paracelsus-Elena-Klinik Kassel unter Anleitung von Frau Prof. Dr. med. B. Mollenhauer in den Jahren 2009 bis 2012.

Dekan: Prof. Dr. med. M. P. Schön

I. Berichterstatterin: Prof. Dr. med. B. Mollenhauer

II. Berichterstatter: Prof. Dr. med. M. Sommer

Tag der mündlichen Prüfung: 11. Dezember 2012 
INHALTSVERZEICHNIS

TABELLEN- UND

Seite

ABBILDUNGSVERZEICHNIS

ABKÜRZUNGSVERZEICHNIS

IV

1. EINLEITUNG

1.1 Der Morbus Parkinson

1.1.1 Epidemiologie

1.1.2 Diagnostik und Symptome

1.1.3 Neuropathologie

1.1.4 Ätiologie und Pathogenese

1.1.5 Biomarker

1.2 Zielsetzungen der (Pilot-)Studie

2. MATERIAL UND METHODEN

2.1 Die DeNoPa-Kassel-Studie

2.1.1 Studiendesign

2.1.2 Ein- und Ausschlusskriterien

2.1.3 Rekrutierung

I

1

1

1

2

5

7

8

16

17

17

17

17

19

2.2 Die Pilotstudie zur DeNoPa-

Kassel-Studie

2.3 Demographische Daten

2.4 Neurologische Untersuchung

2.5 Hirnparenchymsonographie

2.6 Klinische Tests

2.6.1 Riechtest

2.6.2 Schellongtest

2.6.3 Polysomnographie

2.6.4 Neuropsychologische Tests

2.7 Fragebögen

2.8 Liquordiagnostik

2.9 Statistische Analyse

3. ERGEBNISSE

3.1 Demographische Daten

3.2 Neurologische Untersuchung

3.3 Hirnparenchymsonographie

3.4 Klinische Tests

3.4.1 Riechtest

3.4.2 Schellongtest

3.4.3 Polysomnographie

3.4.4 Neuropsychologische Tests

3.5 Fragebögen

3.5.1 NMSQuest

3.5.2 NMSScale

3.5.3 PDQ-39

3.5.4 RBDSQ

3.6 Liquordiagnostik

3.7 Feasibility
19

20

20

22

22

22

22

23

24

24

25

26

28

28

28

33

35

35

37

38

40

40

41

42

43

44

46

47 
4. DISKUSSION

51

4.1 Demographische Daten

51

4.2 Neurologische Untersuchung

51

4.3 Hirnparenchymsonographie

54

4.4 Klinische Tests

56

4.4.1 Riechtest

56

4.4.2 Schellongtest

57

4.4.3 Polysomnographie

58

$\begin{array}{lll}\text { 4.4.4 Neuropsychologische Tests } & 61\end{array}$

4.5 Fragebögen

63

4.5.1 NMSQuest

63

4.5.2 NMSScale

66

4.5.3 PDQ-39

67

4.5.4 RBDSQ

69

4.6 Liquordiagnostik

71

4.7 Feasibility

72

4.8 Schlussfolgerung

73

5. ZUSAMMENFASSUNG

75

6. ANHANG: ABBILDUNGEN 6-1 BIS 6-7

78

7. LITERATURVERZEICHNIS 


\section{TABELLEN- UND ABBILDUNGSVERZEICHNIS}

Seite

Tabelle 1-1: Beispiele für Parkinson-Syndrome 2

Tabelle 1-2: $\quad$ Klinische Diagnosekriterien der United Kingdom Parkinson's

3

Disease Society Brain Bank (nach HUGHES et al. 1992, S. 182)

Tabelle 1-3: Nicht-motorische Symptome des idiopathischen ParkinsonSyndroms (nach CHAUDHURI et al. 2006 a, S. 236)

Tabelle 1-4: $\quad$ Häufige alternative Diagnosen bei Patienten mit früherer Fehldiagnose eines idiopathischen Parkinson-Syndroms

Tabelle 1-5: $\quad$ Potenzielle Biomarker für das idiopathische Parkinson-Syndrom (nach MICHELL et al. 2004)

Tabelle 2-1: Im Rahmen der DeNoPa-Kassel-Studie durchgeführte Untersuchungen

Tabelle 2-2: $\quad$ Aufgabenstellung beim Riechtest mit „Sniffin“ Sticks“

Tabelle 2-3: $\quad$ REM Sleep Behavior Disorder Severity Scale (SIXEL-DORING et al. 2009, S. S395)

Tabelle 3-1: Demographische Daten der Patienten- (PG) und Kontrollgruppe (KG)

Tabelle 3-2: $\quad$ Ergebnisse der Unified Parkinson's Disease Rating Scale (UPDRS), Abschnitt I-IV und Stadien nach HOEHN und YAHR (1967; H\&Y-Stadien) in der Patienten- (PG) und Kontrollgruppe (KG)

Tabelle 3-3: $\quad$ Beeinträchtigungen bei Aktivitäten des täglichen Lebens mit einer Häufigkeit von über zwei Dritteln in der Patientengruppe $(\mathrm{N}=30)$ und deren Ausprägungsgrad in der Unified Parkinson's Disease Rating Scale, Abschnitt II

Tabelle 3-4: $\quad$ Ergebnisse der Hirnparenchymsonographie der Substantia nigra (SN) in der Patienten- (PG) und Kontrollgruppe (KG)

Tabelle 3-5: $\quad$ Echogenität der Substantia nigra (SN)

Tabelle 3-6: $\quad$ Ergebnisse des Riechtests in der Patienten- (PG) und Kontrollgruppe (KG)

Tabelle 3-7: $\quad$ Ergebnisse des neuropsychologischen Screenings mit dem MiniMental-Status-Test (MMST) und Uhrentest in der Patienten- (PG) und Kontrollgruppe (KG)

Tabelle 3-8: $\quad$ Ergebnisse der Fragebogenerhebung in der Patienten- (PG) und Kontrollgruppe (KG)

Tabelle 3-9: $\quad$ Prävalenz nicht-motorischer Symptome in der Patienten- und Kontrollgruppe (in Prozent, gemessen durch den Non-Motor Symptoms Questionnaire)

Tabelle 3-10: $\quad$ Ausprägungsgrad nicht-motorischer Symptome in der

Patientengruppe (gemessen durch die Non-Motor Symptoms Scale; $N=26$ )

Tabelle 3-11: Beeinträchtigung von Aspekten der Lebensqualität (gemessen durch den Parkinson's Disease Questionnaire (PDQ-39)) 
Tabelle 3-12: Vierfeldertafel zur Übereinstimmung des polysomnographischen

Seite

Befundes und des RBD Screening Questionnaires (RBDSQ)

bezüglich des Vorliegens einer REM-Schlaf-Verhaltensstörung (RBD)

Tabelle 3-13: $\quad$ Liquorkonzentrationen von $\beta$-Amyloid (1-42) $_{\text {(A }}(A 42)$, Gesamt-TauProtein (Tau) und hyperphosphoryliertem Tau-Protein (P-Tau) in der Patienten- (PG) und Kontrollgruppe (KG)

Tabelle 3-14: Vollständigkeit der erhobenen Daten

Tabelle 4-1: $\quad$ Mittelwerte des Mini-Mental-Status-Tests verschiedener

Untersuchungen an neu diagnostizierten Parkinson-Patienten und gesunden Kontrollpersonen

Abbildung 2-1: Lage der Kennwerte einer Verteilung im Boxplot

Abbildung 2-2: Vierfeldertafel und Gütekriterien eines diagnostischen Tests

Abbildung 3-1: Häufigkeit der Kardinalsymptome und der bestätigenden Kriterien für das idiopathische Parkinson-Syndrom in der Patientengruppe $(\mathrm{N}=30)$ in \%

Abbildung 3-2: Erkrankungsdauer der 30 Patienten beim Einschluss in die DeNoPa-Kassel-Studie in Monaten

Abbildung 3-3: Prozentuale Verbesserung der Punktzahl im Abschnitt III der Unified Parkinson's Disease Rating Scale bei den 30 Patienten während des Levodopa-Tests

Abbildung 3-4: Anzahl erfüllter bestätigender Kriterien für die klinische Diagnose eines idiopathischen Parkinson-Syndroms entsprechend den Diagnosekriterien der United Kingdom Parkinson's Disease Society Brain Bank (HUGHES et al. 1992) in der Patientengruppe $(\mathrm{N}=30)$

Abbildung 3-5: Boxplots zu den Gesamtpunkten in Abschnitt I (a), II (b) und III (c) der Unified Parkinson's Disease Rating Scale (UPDRS) in der Patienten- $(\mathrm{N}=30)$ und Kontrollgruppe $(\mathrm{N}=10)$

Abbildung 3-6: Häufigkeit der Stadien nach HOEHN und YAHR (1967) in der Patientengruppe

Abbildung 3-7: Boxplots zu den Flächen der rechten und linken Substantia nigra $(\mathrm{SN})$ in der Patienten- $\left(\mathrm{N}_{\text {rechts }}=28, \mathrm{~N}_{\text {links }}=26\right)$ und Kontrollgruppe $(\mathrm{N}=10)$

Abbildung 3-8: Boxplots zu den Ergebnissen der Patienten und Kontrollpersonen im Schwellen-, Diskriminations- und Identifikationstest

Abbildung 3-9: Boxplots zu den TDI-Scores in der Patienten- $(N=29)$ und Kontrollgruppe $(\mathrm{N}=9)$

Abbildung 3-10: Riechfähigkeit der Patienten $(a ; N=29)$ und Kontrollpersonen (b; $\mathrm{N}=9$ ) definiert durch die TDI-Scores

Abbildung 3-11: Differenzen der systolischen (a) und diastolischen (b) Blutdruckwerte der Patienten $(\mathrm{N}=29)$ und Kontrollpersonen $(\mathrm{N}=10)$ im Schellongtest 
Abbildung 3-12: Absoluter und prozentualer Anteil von Personen ohne REM-SchlafVeränderungen, mit REM-Schlaf ohne Atonie (RWA) oder mit einer REM-Schlaf-Verhaltensstörung (RBD) in der Patienten- $(a ; N=30)$ und Kontrollgruppe (b; $N=10)$

Abbildung 3-13: Verteilung der RBD-Grade in der Patienten- und Kontrollgruppe

Abbildung 3-14: Boxplots zu den Konzentrationen von $\beta$-Amyloid ${ }_{(1-42)}(A \beta 42 ; a)$, Gesamt-Tau-Protein (Tau; b) und hyperphosphoryliertem TauProtein (P-Tau; $c)$ im Liquor cerebrospinalis der Patienten $(N=27)$ und Kontrollpersonen $(\mathrm{N}=6)$

Abbildung 3-15: Monatliche Rekrutierungszahlen im Zeitraum von September 2009 bis März 2010

Abbildung 3-16: Hochrechnung der Probandenzahlen bis Ende Dezember 2011 ausgehend von einem Rekrutierungsstand von 36 Patienten und 11 Kontrollpersonen am 31. März 2010 bei einer durchschnittlichen monatlichen Rekrutierung von vier bis acht Patienten bzw. Kontrollpersonen

Abbildung 3-17: Flussdiagramm der von September 2009 bis März 2010 rekrutierten Studienteilnehmer

Abbildung 6-1: Unified Parkinson Disease Rating Scale (UPDRS)

Abbildung 6-2: Uhrentest

Abbildung 6-3: Mini-Mental-Status-Test (MMST)

Abbildung 6-4: Non-Motor Symptoms Questionnaire (NMSQuest)

Abbildung 6-5: Non-Motor Symptoms Scale (NMSScale)

Abbildung 6-6: Parkinson's Disease Questionnaire (PDQ-39)

Abbildung 6-7: RBD Screening Questionnaire (RBDSQ) 


\section{ABKÜRZUNGSVERZEICHNIS}

\begin{tabular}{|c|c|}
\hline$A \beta 42$ & $\beta$-Amyloid $(1-42)$ \\
\hline DeNoPa & Studientitel, Akronym für De-novo-Parkinson \\
\hline EEG & Elektroenzephalographie \\
\hline ELISA & $\begin{array}{l}\text { enzymgekoppelter Immunabsorptionsassay (engl. enzyme linked } \\
\text { immunosorbent assay) }\end{array}$ \\
\hline EMG & Elektromyographie \\
\hline HNA & Hessische/ Niedersächsische Allgemeine Zeitung \\
\hline HPS & Hirnparenchymsonographie \\
\hline H\&Y-Stadium & Stadium nach HOEHN und YAHR (1967) \\
\hline ICSD & International Classification of Sleep Disorders \\
\hline IPS & Idiopathisches Parkinson-Syndrom, Morbus Parkinson \\
\hline L-Dopa & Levodopa, L-3,4-Dihydroxyphenylalanin \\
\hline M. & Krankheit (lat. Morbus) \\
\hline $\mathrm{MCl}$ & $\begin{array}{l}\text { leichte kognitive Beeinträchtigung (engl. minimal cognitive } \\
\text { impairment) }\end{array}$ \\
\hline MMST & Mini-Mental-Status-Test \\
\hline MRT & Magnetresonanztomographie \\
\hline MSA & Multisystematrophie \\
\hline NMS & nicht-motorische(s) Symptom(e) \\
\hline NMSQuest & Non-Motor Symptoms Questionnaire \\
\hline NMSScale & Non-Motor Symptoms Scale \\
\hline $\mathrm{OH}$ & orthostatische Hypotension \\
\hline PDQ-39 & Parkinson's Disease Questionnaire \\
\hline PDQ-39SI & Parkinson's Disease Questionnaire Summary Index \\
\hline PSP & $\begin{array}{l}\text { Progressive supranukleäre Blickparese (engl. progressive supranuclear } \\
\text { palsy) }\end{array}$ \\
\hline P-Tau & hyperphosphoryliertes Tau-Protein \\
\hline p-Wert & $\begin{array}{l}\text { Signifikanzwert; Wahrscheinlichkeit (engl. probability), dass unter der } \\
\text { Annahme, die Nullhypothese sei wahr, die Teststatistik den } \\
\text { beobachteten oder einen extremeren Wert annimmt }\end{array}$ \\
\hline RBD & REM-Schlaf-Verhaltensstörung (engl. REM sleep behaviour disorder) \\
\hline RBDSQ & RBD Screening Questionnaire \\
\hline REM-Schlaf & $\begin{array}{l}\text { Schlafphase mit schnellen Augenbewegungen (engl. rapid eye } \\
\text { movements) }\end{array}$ \\
\hline RWA & REM-Schlaf ohne Atonie (engl. REM sleep without atonia) \\
\hline
\end{tabular}


SN

Tau

TDI-Score

UKPDSBB

UPDRS
Substantia nigra

Gesamt-Tau-Protein

Summenscore abgeleitet aus den Riechtest-Ergebnissen für

Geruchsschwelle (engl. odor threshold), Diskrimination und Identifikation

United Kingdom Parkinson's Disease Society Brain Bank

Unified Parkinson's Disease Rating Scale 


\section{EINLEITUNG}

\subsection{Der Morbus Parkinson}

Obwohl es schon frühere Hinweise auf das Krankheitsbild des Morbus (M.) Parkinson gab (HENNINGSEN 1983), stammt die erste ausführliche Beschreibung der neurologischen Erkrankung vom britischen Arzt und Apotheker James Parkinson (1755-1824). Diese veröffentlichte er 1817 in seinem „Essay on the Shaking Palsy“ (PARKINSON 1817/ 2002). Erst nach Parkinsons Tod, ca. 55 Jahre nach der Erstbeschreibung, wurde der Begriff „maladie de Parkinson“ (frz. für Parkinson-Krankheit) von Jean Martin Charcot (1825-1893), einem französischen Neurologen, geprägt (GOETZ et al. 2001).

James Parkinson beschrieb zu Beginn des 19. Jahrhunderts die Symptome der später nach inm benannten Krankheit folgendermaßen: „Involuntary tremulous motion, with lessened muscular power, in parts not in action and even when supported; with a propensity to bend the trunk forwards, and to pass from a walking to a running pace: the senses and intellects being uninjured" (PARKINSON 1817/ 2002, S. 223). Diese fast 200 Jahre alte Definition entspricht weitgehend den heutigen Vorstellungen zur klinischen Manifestation des $\mathrm{M}$. Parkinson. Allerdings weiß man inzwischen, dass Geist und Sinne sehr wohl von der Erkrankung betroffen sein können (AARSLAND et al. 2008, HAEHNER et al. 2009 a, PRICE et al. 1992).

Für den M. Parkinson bzw. die Parkinson-Krankheit wird synonym der Begriff idiopathisches Parkinson-Syndrom (IPS) verwendet, um inn von anderen Parkinson-Syndromen mit bekannter Ursache abzugrenzen (siehe Tabelle 1-1). Parkinson-Syndrome sind generell definiert durch das obligate Vorliegen einer Bradykinese in Kombination mit mindestens einem der Symptome Rigor, Ruhetremor und/oder posturale Instabilität (OERTEL et al. 2008). Das IPS ist mit ca. 75 \% der häufigste Vertreter (RIEDERER und JELLINGER 1983).

\subsubsection{Epidemiologie}

Das IPS ist nach dem M. Alzheimer die zweithäufigste neurodegenerative Erkrankung (ALVES et al. 2008). Die Prävalenz wird für Europa auf etwa 100 bis 250/100.000 Einwohner, die Inzidenz auf 11 bis 19/100.000 Einwohner pro Jahr geschätzt (VON CAMPENHAUSEN et al. 2005). Daraus folgt, dass in Deutschland bei einer Einwohnerzahl von 81.752.000 (STATISTISCHES BUNDESAMT DEUTSCHLAND, Stand: 31.12.2010) bis zu 200.000 Menschen betroffen sind und jährlich bis zu 15.500 neu erkranken. 


\section{Tabelle 1-1: Beispiele für Parkinson-Syndrome}

a. Idiopathisches Parkinson-Syndrom (Morbus Parkinson)

b. Familiäres Parkinson-Syndrom

c. Sekundäre (symptomatische) Parkinson-Syndrome:

- vaskulär (subkortikale vaskuläre Enzephalopathie)

- medikamenteninduziert (z. B. durch Neuroleptika, Antidepressiva, Antiemetika)

- $\quad$ toxisch (z. B. durch MPTP, Kohlenmonoxid, Mangan)

- posttraumatisch

- metabolisch (z. B. bei Morbus Wilson)

- tumorbedingt

- bei Normaldruckhydrozephalus

d. Atypische Parkinson-Syndrome (im Rahmen anderer neurodegenerativer Erkrankungen):

- Multisystematrophie

- Progressive supranukleäre Blickparese

- Kortikobasale Degeneration

(MPTP: 1-Methyl-4-phenyl-1,2,3,6-tetrahydropyridin)

Allerdings steigt die Häufigkeit mit dem Alter stark an. Unter den über 60-Jährigen liegt die Prävalenz in Europa bei bis zu 1.500/100.000 Einwohner und die Inzidenz bei ca. 350/100.000 (VON CAMPENHAUSEN et al. 2005). Eine deutsche Prävalenzstudie von TRENKWALDER et al. (1995) ergab eine Prävalenz von 713/100.000 bei über 65-Jährigen. Bei fast 17.000.000 Einwohnern in dieser Altersgruppe (STATISTISCHES BUNDESAMT DEUTSCHLAND; Stand: 2010) entspricht das etwa 120.000 Patienten.

Männer scheinen zumindest in westlichen Populationen und bei einem Erkrankungsalter von über 60 Jahren 1,5-mal häufiger an IPS zu erkranken als Frauen (TAYLOR KSM et al. 2007). Das mittlere Erkrankungsalter liegt zwischen 60 und 65 Jahren (O'SULLIVAN et al. 2008, HUGHES et al. 1992). Nur $4 \%$ der Patienten erkranken vor dem 50. Lebensjahr (VAN DEN EEDEN et al. 2003). Da das IPS eine Erkrankung des höheren Lebensalters ist, ist zu erwarten, dass im Zuge der Veränderungen der Altersstruktur die Zahl der ParkinsonPatienten steigen wird. Die mittlere Erkrankungsdauer beträgt etwa 12 Jahre (HELY et al. 2005).

\subsubsection{Diagnostik und Symptome}

Diagnostisch stehen die motorischen Symptome des IPS im Vordergrund: Die international gültigen Diagnosekriterien der United Kingdom Parkinson's Disease Society Brain Bank (UKPDSBB-Kriterien; HUGHES et al. 1992) fordern das Vorliegen einer Bradykinese zusammen mit mindestens einem der drei weiteren Kardinalsymptome der ParkinsonSyndrome Rigor, Ruhetremor oder posturale Instabilität. Je nach vorherrschenden Kardinalsymptomen werden verschiedene Unterformen differenziert, die sich unter anderem 
in ihrem Progress und dem Erkrankungsalter unterscheiden: der akinetisch-rigide Typ, der Tremor-dominante Typ und ein Mischtyp (KORCHOUNOV et al. 2004, JANKOVIC et al. 1990). Die weiteren Diagnoseschritte beinhalten den Ausschluss von Hinweisen auf ein Parkinson-Syndrom bekannter Ursache sowie die Erfassung supportiver Kriterien für das IPS (siehe Tabelle 1-2).

Tabelle 1-2: Klinische Diagnosekriterien der United Kingdom Parkinson's Disease Society Brain Bank (nach HUGHES et al. 1992, S. 182)

\section{Schritt 1: Diagnosekriterien für Parkinson-Syndrome}

Bradykinese (Verlangsamung bei der Initiierung willkürlicher Bewegungen mit zunehmender Reduktion von Geschwindigkeit und Amplitude bei repetitiven Bewegungen) und mindestens eines der folgenden Symptome

- Rigor

- Ruhetremor (4-6 Hertz)

- posturale Instabilität (nicht durch primär visuelle, vestibuläre, zerebelläre oder propriozeptive Störungen verursacht)

Schritt 2: Ausschlusskriterien für den Morbus Parkinson

- rezidivierende zerebrale ischämische Insulte in der Vorgeschichte assoziiert mit einem Progress der Parkinson-Symptomatik

- rezidivierende Schädel-Hirn-Traumata in der Vorgeschichte

- definitive Enzephalitis in der Vorgeschichte

- okulogyre Krisen

- Behandlung mit Neuroleptika zum Zeitpunkt des Symptombeginns

- mehr als ein betroffener Verwandter

- anhaltende Remission

- streng einseitige Symptome nach drei Jahren Verlauf

- vertikale Blickparese

- zerebelläre Zeichen

- frühe schwere autonome Störungen

- frühe schwere Demenz mit Störung von Gedächtnis, Sprache und Praxie

- positives Babinski-Zeichen

- Nachweis eines zerebralen Tumors oder Hydrocephalus communicans in der Computertomographie

- Nichtansprechen auf hohe Dosen Levodopa (trotz Ausschluss einer Malabsorption)

- MPTP (1-Methyl-4-phenyl-1,2,3,6-tetrahydropyridin)-Exposition

Schritt 3: supportive Kriterien für den Morbus Parkinson

(mindestens drei für eine sichere klinische Diagnose erforderlich)

- einseitiger Beginn

- Ruhetremor

- Fortschreiten der Erkrankung

- persistierende Asymmetrie im Krankheitsverlauf

- exzellentes Ansprechen (70-100 \%) auf Levodopa

- schwere Levodopa-induzierte Chorea

- Ansprechen auf Levodopa für mindestens fünf Jahre

- mindestens zehn Jahre klinischer Verlauf

Das IPS verläuft chronisch progredient. Es manifestiert sich sowohl in der kranialen (z. B. Hypomimie, Dysarthrie, Hypophonie, Dysphagie), der axialen (z. B. Haltungsstörungen wie Kamptokormie, Gang- und Standunsicherheit) als auch in der Extremitätenmotorik (z. B. 
Mikrographie, Tremor, Bradydiadochokinese, Störung der Feinmotorik, verminderter Armschwung, Freezing) (JANKOVIC 2008). Die motorischen Symptome beginnen typischerweise einseitig und bleiben asymmetrisch (UITTI et al. 2005). Sie sind häufig zunächst unspezifisch und subtil und werden daher nicht beachtet oder missinterpretiert. Erst im Rückblick bringen Patienten und Angehörige diese mit der Erkrankung in Verbindung. Bis der Verdacht auf IPS gestellt wird, vergehen nicht selten mehrere Monate und unter Umständen sogar Jahre (LEES et a. 2009, HOEHN und YAHR 1967, WILLIAMS und LEES 2009).

Abgesehen von den motorischen Symptomen bemerkte schon James Parkinson weitere Probleme wie Obstipation, Harn- und Stuhlinkontinenz oder Hypersalivation (PARKINSON $1817 /$ 2002). Neben diesen autonomen bzw. gastrointestinalen Symptomen wurde inzwischen ein breites Spektrum nicht-motorischer Symptome (NMS) beobachtet und diskutiert (siehe Tabelle 1-3). Dazu gehören, entgegen der Annahme von James Parkinson, auch kognitive Defizite. Diese reichen von minimalen Auffälligkeiten in spezifischen neuropsychologischen Tests bis zur manifesten Demenz bei Parkinson, deren diagnostische Kriterien Einschränkungen des täglichen Lebens voraussetzten (AARSLAND et al. 2009 b, DUBOIS et al. 2007 a). In verschiedenen Untersuchungen wurden bereits bei neu diagnostizierten Patienten kognitive Einschränkungen festgestellt (MUSLIMOVI et al. 2005; AARSLAND et al. 2009 b; ELGH et al. 2009).

Tabelle 1-3: Nicht-motorische Symptome des idiopathischen Parkinson-Syndroms (nach CHAUDHURI et al. 2006 a, S. 236)

- neuro-psychiatrische Symptome

(z. B. Depression, Apathie, Angststörungen, Halluzinationen, Demenz)

- Schlafstörungen

(z. B. REM-Schlaf-Verhaltensstörung, Restless-Legs-Syndrom, Tagesmüdigkeit)

- autonomel gastrointestinale Symptome

(z. B. Störung der Harnblasenfunktion, sexuelle Dysfunktion, Hyperhidrose, orthostatische Hypotension, Sialorrhö, Obstipation, Stuhlinkontinenz, Nausea, Dysphagie)

- sensorische Symptome

(z. B. Schmerzen, Parästhesien, Geruchsstörungen)

- andere Symptome

(z. B. Diplopie, Seborrhö, Gewichtsverlust)

(REM-Schlaf: Schlafphase mit schnellen Augenbewegungen)

NMS sind ein häufiges Problem fortgeschrittener Krankheitsstadien (MARTINEZ-MARTIN et al. 2007, HELY et al. 2005). Eine retrospektive Analyse der Fallberichte von 433 Patienten mit pathologisch gesicherter Parkinson-Diagnose ergab zudem, dass die ursprünglichen Beschwerden, die vor der Diagnosestellung zur Konsultation eines Arztes geführt hatten, in 
$21 \%$ ausschließlich nicht-motorischer Art waren. Am häufigsten lagen Schmerzen, Miktionsstörungen, Angst und Depression vor (O'SULLIVAN et al. 2008). SHULMAN LM et al. (2002) stellten allerdings fest, dass Neurologen während routinemäßiger Arztbesuche vorhandene NMS nur bei etwa der Hälfte ihrer Patienten erkannten.

Einige der NMS, besonders Hyposmie, REM-Schlaf-Verhaltensstörung (RBD, engl. REM sleep behaviour disorder; REM-Schlaf: Schlafphase mit schnellen Augenbewegungen) und ausgeprägte Tagesmüdigkeit, Obstipation und Depression scheinen der typischen motorischen Manifestation des IPS vorauszugehen (TOLOSA et al. 2009). Trotzdem basiert die Diagnose bisher auf dem Erscheinen motorischer Symptome. Auch die orthostatische Hypotension $(\mathrm{OH})$ wurde von einigen Autoren als Frühsymptom des IPS diskutiert (BONUCCELLI et al. 2003, KAUFMANN et al. 2004, GOLDSTEIN 2006), obwohl eine frühzeitig im Verlauf auftretende $\mathrm{OH}$ als differenzialdiagnostischer Hinweis für die Multisystematrophie (MSA) gilt („Leitlinie Parkinson-Syndrome“, OERTEL et al. 2008). Depression, Müdigkeit, Schmerzen und autonome Störungen wie Inkontinenz oder Obstipation gehören laut RAHMAN et al. (2008) zu den Symptomen mit dem größten Einfluss auf die Lebensqualität der Patienten. Die NMS rücken daher zunehmend in den Blick, wenn es darum geht, die Effektivität neuer Therapien zu beurteilen (HONIG et al. 2009, ALESSANDRO et al. 2010). LANGSTON postulierte 2006 „parkinsonism only represents the tip of the iceberg that makes up this diverse disease" (S. 591) und forderte daher ein Umdenken bezüglich NMS und die Integration dieser in diagnostische und therapeutische Überlegungen.

Vor diesem Hintergrund entwickelten CHAUDHURI et al. (2006 b und 2007) einen Screening-Fragebogen (NMSQuest) und eine Skala (NMSScale) zur Erfassung eines breiten Spektrums der NMS des IPS aus den Bereichen kardiovaskuläres System, Schlaf, Stimmung/ Kognition, Wahrnehmungsprobleme/ Halluzinationen, Aufmerksamkeit/ Gedächtnis, gastrointestinaler Trakt, Miktion, Sexualfunktionen und Verschiedenes. Der Fragebogen soll Patienten die Möglichkeit geben, ihre nicht-motorischen Probleme zu äußern und gleichzeitig die ärztliche Aufmerksamkeit auf deren Vorliegen lenken, sodass gegebenenfalls diagnostische und therapeutische Maßnahmen ergriffen werden können (CHAUDHURI et al. 2006 b).

\subsubsection{Neuropathologie}

Charakteristisch für das IPS ist ein selektiver Verlust Melanin-haltiger Neurone in der Pars compacta der Substantia nigra (SN) (DAMIER et al. 1999, BERNHEIMER et al. 1973). Diese Neurone kommunizieren über den Neurotransmitter Dopamin mit striatalen Neuronen und haben im Zusammenspiel mit dem motorischen Kortex und den Basalganglien eine 
bewegungsfördernde Funktion (ALBIN et al. 1989). Die Rate des Zelluntergangs in der SN hat sich im Vergleich zu Kontrollpersonen bei Parkinson-Patienten von 4,7\% auf $45 \%$ im Jahrzehnt erhöht (FEARNLEY und LEES 1991). Bis die ersten motorischen ParkinsonSymptome auftreten, sind bereits $50 \%$ der nigralen Neurone untergegangen (ebd.) und die Dopaminkonzentration im Striatum ist um 70-80 \% reduziert (BERNHEIMER et al. 1973). Der Neuronenverlust betrifft allerdings nicht nur die SN (JELLINGER 1987).

In diversen Rückenmarks-, Hirnstamm- und Kortex-Regionen (BRAAK 2003), aber auch im peripheren (DEN HARTOG JAGER und BETHLEM 1960) und enterischen (WAKABAYASHI et al. 1988) Nervensystem finden sich typische intraneuronale Einschlüsse. Diese wurden erstmals 1913 von LEWY im Kern der Substantia innominata und im dorsalen Vaguskern entdeckt und daher später als Lewy-Körper bezeichnet. Hauptbestandteil der Aggregate ist abnormales a-Synuclein, ein präsynaptisches neuronales Protein (SPILLANTINI et al. 1997, IWAI et al. 1995). a-Synuclein-positive Einschlüsse kommen auch bei anderen Erkrankungen vor, die man daher Synukleinopathien nennt. Zu dieser Gruppe gehören unter anderem die Demenz vom Lewy-Körper-Typ und die MSA (JELLINGER 2003). Für letztere sind $\alpha-$ Synuclein-Einschlüsse nicht in Neuronen, sondern in Oligodendrozyten kennzeichnend (WENNING et al. 2008).

Die pathologische Diagnose des IPS basiert auf „clear depletion of brain stem pigmented neurons with Lewy bodies in some of the remaining nerve cells" (HUGHES et al. 1992, S. 181). Ob die Lewy-Körper allerdings zur Degeneration der Neurone führen oder eher einen protektiven oder adaptiven Prozess darstellen, ist umstritten (WAKABAYASHI et al. 2007). Beobachtungen von MC NAUGHT et al. (2002) deuten darauf hin, dass die Bildung von Lewy-Körpern eine Reaktion auf das Vorhandensein großer Mengen abnormaler Proteine in der Zellperipherie ist. KRAMER und SCHULZ-SCHAEFFER (2007) postulierten, dass bei der Demenz vom Lewy-Körper-Typ nicht die juxtanukleär lokalisierten Lewy-Körper, sondern kleine präsynaptische $\alpha$-Synuclein-Aggregate zu einer synaptischen Dysfunktion führten und für die neurodegenerativen Symptome verantwortlich seien. Die Akkumulation dieser Aggregate sei assoziiert mit einem Verlust dendritischer Spines, einem Vorgang, der auch bei striatalen Neuronen von Parkinson-Patienten beobachtet wurde (MC NEILL et al. 1988). Daraus schlossen die Autoren, ihr Konzept könne für beide Erkrankungen Relevanz haben. Aktuellen Hypothesen zufolge beginnt die Pathologie des IPS im Nucleus olfactorius anterior und/ oder dem dorsalen motorischen Vaguskern und breitet sich vom Hirnstamm systematisch in höhere Regionen einschließlich des Kortex aus. In diesen aufsteigenden Prozess seien bestimmte vulnerable dopaminerge und nicht-dopaminerge Nervenzelltypen involviert (BRAAK 2003). Da alle betroffenen Regionen durch neuronale Bahnen miteinander verbunden sind, sei es denkbar, dass ein neurotropes Pathogen über die Nasenschleimhaut oder den Magen-Darm-Trakt in das Nervensystem eindringe und sich von dort intraneuronal 
auf dem oben genannten Weg ausbreite (BRAAK und DEL TREDICI 2010). Dem gerichteten Verlauf entsprechend wurde von BRAAK (2003) eine pathologische Stadieneinteilung etabliert. Die SN ist erst ab Stadium 3 beteiligt. Das Auftreten von NMS vor den motorischen Kardinalsymptomen des IPS erklärt sich folglich durch das Vorhandensein pathologischer Veränderungen im unteren Hirnstamm in Stadium 1 und 2.

Altersabhängig können bei bis zu $13 \%$ der Personen ohne klinisch manifestes IPS im Hirnstamm Lewy-Körper nachgewiesen werden. Es besteht die Vorstellung, dass diese inzidentelle Lewy-Körper-Erkrankung einem präsymptomatischen IPS entspricht (GIBB und LEES 1988). Eine Studie von PARKKINEN et al. (2005) zur klinischen Relevanz der aSynuclein-Pathologie zeigte allerdings, dass deren Verteilung und Ausmaß keine verlässliche Vorhersage über das Vorhandensein extrapyramidaler Symptome zulässt. In einigen Fällen waren trotz ausgeprägter pathologischer Veränderungen im Hirnstamm (einschließlich der SN) und/ oder kortikalen Regionen keine Symptome aufgetreten. Umgekehrt gab es auch Fälle mit Symptomen, bei denen im Widerspruch zu den BraakStadien keine a-Synuclein-positiven Einschlüsse in der SN identifiziert werden konnten. Daraus folgerten die Autoren, es müsse bisher unerkannte Pathologien mit entscheidender Bedeutung für das IPS geben. Außerdem sind inzwischen familiäre Parkinson-Formen bekannt, die mit reduzierten Neuronenzahlen in der SN einhergehen, ohne dass LewyKörper nachweisbar wären (TAKAHASHI et al. 1994).

Die "Sydney Multicenter Study of Parkinson's disease” ergab, dass nur ein Teil der Patienten, und zwar solche mit einem relativ frühen Krankheitsbeginn, den typischen stadienhaften Verlauf zeigten, wie inn die Braak-Stadien vorhersagen. Daneben gebe es zwei Gruppen von Patienten mit abweichender Pathologie. Die eine Gruppe habe eine Demenz-dominante Form mit schwerer neokortikaler Beteiligung. Die andere zeige einen komplexeren Krankheitsverlauf mit spätem Beginn, kürzerem Überleben und pathologisch höheren Lewy-Körper-Zahlen neben zusätzlichen Pathologien (HALLIDAY et al. 2008).

\subsection{4 Ätiologie und Pathogenese}

Trotz intensiver Forschung weiß man noch sehr wenig über Ätiologie und Pathogenese des IPS. Der wichtigste bekannte Risikofaktor ist das Alter (DE RIJK et al. 1997, DRIVER et al. 2009, VAN DEN EEDEN et al. 2003). Da Parkinson-Patienten im Vergleich zu Kontrollpersonen häufiger betroffene Familienangehörige haben (KURZ et al. 2003) und das Risiko, an Parkinson zu erkranken, bei Verwandten ersten Grades erhöht ist (PAYAMI et al. 1994), wurde eine genetische Ursache hypothesiert. Außerdem wurden prädisponierende Mutationen, z. B. im für die Leucine Rich Repeat Kinase 2 oder die Glukozerebrosidase kodierenden Gen, diskutiert (WIDER et al. 2010). Andererseits fanden TANNER et al. (1999) 
in einer Zwillingsstudie (zumindest bei Personen mit einem Krankheitsbeginn nach dem 50. Lebensjahr) keine höhere Konkordanz für das IPS bei monozygoten im Vergleich zu dizygoten Zwillingen, was als Argument gegen eine erblich bedingte Komponente in der Ätiologie des IPS gewertet wurde.

Epidemiologisch wurden diverse Umwelteinflüsse als Risikofaktoren identifiziert. Dazu gehören berufliche Faktoren, wie die Beschäftigung in der Landwirtschaft und die Exposition gegenüber Pestiziden, aber auch der Konsum von Brunnenwasser und Bewusstlosigkeit durch Kopftrauma (ZORZON et al. 2002, DICK et al. 2007, ASCHERIO et al. 2006). Rauchen und Kaffeekonsum sind hingegen assoziiert mit einem erniedrigten Erkrankungsrisiko (RITZ et al. 2007, FALL et al. 1999). Bei einigen dieser Faktoren herrscht allerdings keine Einigkeit. So fanden TAYLOR CA et al. (1999) z. B. keine Verbindung zwischen Pestiziden-Exposition und Erkrankungsrisiko.

Für den Zelltod beim IPS werden verschiedene pathogenetische Mechanismen verantwortlich gemacht: oxidativer Stress, Exzitotoxizität, Mangel an neurotrophischen Faktoren, mitochondriale Dysfunktion, Neuroinflammation und Apoptose (OLANOW und TATTON 1999). Bei einigen der postulierten Faktoren könnte es sich auch um Sekundärfolgen des Krankheitsprozesses handeln, da sie bei Personen mit inzidentellen Lewy-Körpern und Zellverlust nicht nachweisbar waren (DEXTER et al. 1994). Wenn allerdings, wie von KRAMER und SCHULZ-SCHAEFFER (2007) postuliert, nicht der Verlust von Neuronen, sondern eine präsynaptische Dysfunktion für die neurodegenerativen Symptome des IPS verantwortlich ist (vgl. Kapitel 1.1.3), so könnten Aspekte wie der oxidative Stress laut SCHULZ-SCHAEFFER (2010) möglicherweise die Initiation der aSynuclein-Aggregation in der Präsynapse oder die Vulnerabilität bestimmter Neurone für die Bildung dieser Aggregate erklären.

\subsubsection{Biomarker}

Biomarker sind definiert als Merkmale, die objektiv gemessen und ausgewertet werden können und einen Indikator für normale biologische Prozesse, pathologische Prozesse oder für das Ansprechen auf eine therapeutische Intervention darstellen (BIOMARKERS DEFINITIONS WORKING GROUP 2001).

Für die häufigste neurodegenerative Erkrankung, den M. Alzheimer, haben sich bereits diagnostische Biomarker aus dem Liquor cerebrospinalis (erniedrigtes $\beta$-Amyloid ${ }_{(1-42)}(A \beta 42)$, erhöhtes Gesamt-Tau-Protein (Tau) und hyperphosphoryliertes Tau-Protein (P-Tau)) sowie bildgebende Verfahren (Nachweis einer Atrophie des medialen Temporallappens in der Magnetresonanztomographie (MRT) oder bilateral reduzierter Glukose-Metabolismus in temporal-parietalen Regionen in der Positronenemissionstomographie) etabliert (DUBOIS et 
al. 2007 b). Außerdem wurde beispielsweise ein Polymorphismus im Apolipoprotein E identifiziert, der einen Risikofaktor für die Alzheimer-Demenz darstellt (TANG et al. 1998) und die Diagnosestellung unterstützt (MAYEUX et al. 1998).

Bis heute gibt es allerdings keine anerkannten und ausreichend validierten Biomarker für das IPS. Die Diagnostik basiert wie oben beschrieben bisher ausschließlich auf anamnestischen Angaben, der klinischen Untersuchung sowie dem Ansprechen auf dopaminerge Medikation. Verglichen mit dem Goldstandard, der pathologischen Diagnose, liegt die Richtigkeit der entsprechend der UKPDSBB-Kriterien gestellten Diagnose in fortgeschrittenen Krankheitsstadien bei etwa $90 \%$ (HUGHES et al. 2001). Neurologen mit besonderer Erfahrung auf dem Gebiet der Bewegungsstörungen erkennen bis zu 98,6 \% der Patienten mit IPS korrekt (HUGHES et al. 2002). In der Allgemeinbevölkerung sind Fehldiagnosen allerdings häufig: Patienten in Nord-Wales, die von ihrem Allgemeinarzt ParkinsonMedikamente verschrieben bekamen, hatten nur zu 74 \% überhaupt ein Parkinson-Syndrom (MEARA et al. 1999) und bei $15 \%$ der Londoner Einwohner mit IPS musste bei strikter Anwendung der klinischen Kriterien die Diagnose verworfen werden (SCHRAG et al. 2002 b). Die Erkrankungen, die in diesen Studien und denen von HUGHES et al. (2001 und 2002) am häufigsten fälschlicherweise für ein IPS gehalten wurden, sind in Tabelle 1-4 zusammengestellt.

Besonders im frühen Krankheitsverlauf wird die Differenzierung des IPS von anderen Parkinson-Syndromen oder Tremorerkrankungen erschwert und die Diagnosestellung verzögert, wenn die Symptome noch wenig ausgeprägt sind und sich überschneiden (TOLOSA et al. 2006). Darüber hinaus können Kardinalsymptome des IPS bei älteren Erwachsenen auch im Zusammenhang mit anderen häufigen Erkrankungen, wie dem Diabetes mellitus, auftreten (ARVANITAKIS et al. 2004).

HUGHES et al. (2002) stellten bei einer Aufarbeitung von 143 Fällen von Parkinson-Syndrom fest, dass bei über 30 \% der Patienten die klinische Diagnose im Verlauf revidiert wurde und die endgültige Diagnose erst nach bis zu 19 Jahren Krankheit feststand. Eine prospektive klinisch-pathologische Studie von RAJPUT et al. (1991 b) ergab, dass die initiale Diagnose eines IPS nur bei $65 \%$ der Patienten mit der pathologischen Diagnose übereinstimmte. Eine ähnliche Beobachtung machten JANKOVIC et al. (2000) bei einer Medikamenten-Studie mit 800 frühen Parkinson-Patienten: Bei 8,1\% der Patienten wurde die Diagnose im Zuge der Verlaufsuntersuchungen korrigiert. Eine Autopsie wurde allerdings nur bei achtzehn der Patienten durchgeführt und nur vier der den Autoren bekannten dreizehn Ergebnisse bestätigten ein IPS. Diese Erkenntnisse unterstreichen die Notwendigkeit, die (Früh-)Diagnostik durch andere als nur klinische Parameter zu ergänzen. 
Tabelle 1-4: Häufige alternative Diagnosen bei Patienten mit früherer Fehldiagnose eines idiopathischen Parkinson-Syndroms

\begin{tabular}{|c|c|c|}
\hline \multicolumn{3}{|l|}{ Atypische Parkinson-Syndrome } \\
\hline \multirow[t]{2}{*}{ - Multisystematrophie } & $60 \%$ & (HUGHES et al. 2001) \\
\hline & $57 \%$ & (HUGHES et al. 2002) \\
\hline \multirow{2}{*}{$\begin{array}{l}\text { - Progressive supranukleäre } \\
\text { Blickparese }\end{array}$} & $20 \%$ & (HUGHES et al. 2001) \\
\hline & & (SCHRAG et al. 2002 b) \\
\hline \multicolumn{3}{|l|}{ Tremorerkrankungen } \\
\hline - $\quad$ Nicht-Parkinsontremor & $20 \%$ & (SCHRAG et al.(2002 b) \\
\hline - Essenzieller Tremor & k. A. & (MEARA et al. 1999) \\
\hline \multicolumn{3}{|l|}{ Sekundäre Parkinson-Syndrome } \\
\hline \multirow[t]{2}{*}{ - $\quad$ vaskuläres Parkinson-Syndrom } & $30 \%$ & (SCHRAG et al. 2002 b) \\
\hline & k. A. & (MEARA et al. 1999) \\
\hline \multicolumn{3}{|l|}{ Demenzen } \\
\hline - $\quad$ Alzheimer-Demenz & k. A. & (MEARA et al. 1999) \\
\hline
\end{tabular}

Die Ergebnisse stammen aus den klinischen Studien von MEARA et al. (1999) und SCHRAG et al. (2002 b) und den klinisch-pathologischen Studien von HUGHES et al. (2001 und 2002). Der Prozentwert gibt den Anteil der Diagnose an der Gesamtzahl von Fehldiagnosen in der jeweiligen Untersuchung wieder (k. A.: keine Angabe).

Die frühe Diagnose oder möglichenfalls die Identifikation von Risikopersonen in einem präsymptomatischen Stadium ist beim IPS besonders wichtig, da die pathologischen Veränderungen zum Zeitpunkt der motorischen Erstmanifestation schon weit vorangeschritten sind (FEARNLEY und LEES 1991, BERNHEIMER et al. 1973). Die Wirksamkeit zukünftiger neuroprotektiver oder progressionshemmender Therapien in einem so fortgeschrittenen Stadium des degenerativen Prozesses erscheint fragwürdig (BECKER et al. 2002).

Neben diagnostischen Biomarkern werden Marker für das IPS gebraucht, um den Verlauf der Erkrankung vorherzusagen (prognostische Marker) und um den pathologischen oder klinischen Progress zu überwachen (Monitoring-Marker) und daran die Effizienz neuer Behandlungsmethoden zu messen (MICHELL et al. 2004). In Tabelle 1-5 ist eine Auswahl von Biomarker-Kandidaten zusammengestellt.

Gegenstand der vorliegenden Arbeit sind die potenziellen Biomarker:

- Hyperechogenität der SN,

- Geruchsstörung,

- RBD,

- Konzentrationen der Liquorproteine Tau, P-Tau und Aß42.

Sie werden im Folgenden genauer vorgestellt. 
- EINLEITUNG -

Tabelle 1-5: Potenzielle Biomarker für das idiopathische Parkinson-Syndrom (nach MICHELL et al. 2004)

\begin{tabular}{|c|c|c|c|}
\hline Untersuchung & Messparameter & Befund bei IPS & Marker-Typ \\
\hline \multicolumn{4}{|l|}{ (1) klinische Tests } \\
\hline $\begin{array}{l}\text { psychiatrischel neuro- } \\
\text { psychologische Tests } \\
\text { (MONTGOMERY et al. 2000; } \\
\text { WILLIAMS-GRAY et al. 2007, } \\
\text { TODES und LEES 1985) }\end{array}$ & $\begin{array}{l}\text { neuropsychiatrische } \\
\text { Symptome, kognitive } \\
\text { Defizite, } \\
\text { Persönlichkeits- } \\
\text { merkmale }\end{array}$ & $\begin{array}{l}\text { Z. B. Depression, } \\
\text { Einschränkungen der } \\
\text { Wortflüssigkeit/ der } \\
\text { exekutiven Fähigkeiten }\end{array}$ & $\begin{array}{l}\text { diagnostisch/ } \\
\text { prognostisch }\end{array}$ \\
\hline $\begin{array}{l}\text { motorische Tests } \\
\text { (HAAXMA et al. 2010; } \\
\text { TETRUD 1991) }\end{array}$ & $\begin{array}{l}\text { Feinmotorik } \\
\text { Schrift } \\
\text { visuomotorische } \\
\text { Kontrolle }\end{array}$ & $\begin{array}{l}\text { Ungeschicklichkeit/ } \\
\text { Bradykinese } \\
\text { Mikrographie } \\
\text { eingeschränkte } \\
\text { Kontrolle von } \\
\text { Bewegungsrichtung und } \\
\text {-geschwindigkeit }\end{array}$ & diagnostisch \\
\hline $\begin{array}{l}\text { neurophysiologische Tests } \\
\text { (VALLS-SOLÉ und } \\
\text { VALLDEORIOLA 2002; } \\
\text { FILIPOVIĆ et al. 2001) }\end{array}$ & $\begin{array}{l}\text { Muskelaktivität } \\
\text { Reaktionszeit } \\
\text { Bereitschaftspotenzial }\end{array}$ & $\begin{array}{l}\text { Ruhetremor mit 4-6 } \\
\text { Hertz, erhöhte } \\
\text { Grundaktivität } \\
\text { verzögerte Reaktion } \\
\text { verminderte Amplitude } \\
\text { und Steigung }\end{array}$ & $\begin{array}{l}\text { diagnostisch/ } \\
\text { Monitoring }\end{array}$ \\
\hline $\begin{array}{ll}\text { - } & \text { Riechtests } \\
\text { (BERENDSE und PONSEN } \\
\text { 2009; HAEHNER et al. } 2009 \text { b; } \\
\text { BOHNEN et al. 2008) }\end{array}$ & $\begin{array}{l}\text { Geruchsschwelle, } \\
\text { Identifikations-/ } \\
\text { Diskriminationsfähigkeit }\end{array}$ & Hyposmie & diagnostisch \\
\hline $\begin{array}{l}\text { - Sehtests } \\
\text { (BÜTTNER et al. 1995; } \\
\text { PRICE et al. 1992) }\end{array}$ & $\begin{array}{l}\text { Farbsehen } \\
\text { Kontrastempfindlichkeit }\end{array}$ & $\begin{array}{l}\text { gestörte } \\
\text { Farbdiskrimination } \\
\text { erhöhte } \\
\text { Kontrastschwelle }\end{array}$ & diagnostisch \\
\hline $\begin{array}{ll}- & \text { Polysomnographie } \\
\text { (STIASNY-KOLSTER et al. } \\
\text { 2005; SCHENCK et al. 1996) }\end{array}$ & Schlafverhalten & $\begin{array}{l}\text { REM-Schlaf- } \\
\text { Verhaltensstörung }\end{array}$ & diagnostisch \\
\hline \multicolumn{4}{|c|}{ (2) Funktionsbildgebung (RAVINA et al. 2005; BROOKS et al. 2003) } \\
\hline - $\quad$ PET mit $\left[{ }^{18}\right.$ F]Dopa & $\begin{array}{l}\text { Funktion dopaminerger } \\
\text { Nervenendigungen } \\
\text { (Decarboxylase- } \\
\text { aktivität) }\end{array}$ & $\begin{array}{l}\text { neuronale Dysfunktion } \\
\rightarrow \text { erniedrigte striatale } \\
\text { Anreicherung }\end{array}$ & \multirow[t]{3}{*}{$\begin{array}{l}\text { diagnostisch/ } \\
\text { Monitoring }\end{array}$} \\
\hline $\begin{array}{ll}- & \text { PET mit }\left[{ }^{11} \mathrm{C}\right] \mathrm{DTBZ}\end{array}$ & $\begin{array}{l}\text { Funktion dopaminerger } \\
\text { Nervenendigungen } \\
\text { (Vesikulärer Monoamin- } \\
\text { transporter, Typ 2) }\end{array}$ & $\begin{array}{l}\text { neuronale Dysfunktion } \\
\rightarrow \text { erniedrigte striatale } \\
\text { Anreicherung }\end{array}$ & \\
\hline - $\quad$ SPECT mit $\left[{ }^{123} I\right] \beta-C I T$ & $\begin{array}{l}\text { Funktion dopaminerger } \\
\text { Nervenendigungen } \\
\text { (Dopamintransporter) }\end{array}$ & $\begin{array}{l}\text { neuronale Dysfunktion } \\
\rightarrow \text { erniedrigte striatale } \\
\text { Anreicherung }\end{array}$ & \\
\hline $\begin{array}{ll}\text { - } & \text { Szintigraphie mit }\left[{ }^{131} \text { I]MIBG }\right. \\
\text { (BRAUNE 2001) }\end{array}$ & $\begin{array}{l}\text { Funktion adrenerger } \\
\text { Neurone und Zellen }\end{array}$ & $\begin{array}{l}\text { Dysfunktion } \\
\text { postganglionärer, } \\
\text { sympathischer Neurone } \\
\rightarrow \text { erniedrigte kardiale } \\
\text { Anreicherung }\end{array}$ & diagnostisch \\
\hline \multicolumn{4}{|l|}{ (3) Strukturelle Bildgebung } \\
\hline $\begin{array}{l}\text { Hirnparenchym- } \\
\text { sonographie } \\
\text { (WALTER et al. 2004; } \\
\text { SOMMER et al. 2004) } \\
\end{array}$ & $\begin{array}{l}\text { Echogenität der } \\
\text { Substantia nigra }\end{array}$ & Hyperechogenität & diagnostisch \\
\hline
\end{tabular}




\section{Fortsetzung Tabelle 1-5: Potenzielle Biomarker}

\begin{tabular}{|c|c|c|c|}
\hline Untersuchung & Messparameter & Befund bei IPS & Marker-Typ \\
\hline \multicolumn{4}{|l|}{ (4) Laboranalysen } \\
\hline $\begin{array}{l}\alpha-S y n u c l e i n \\
\text { (TOKUDA et al. 2006; } \\
\text { MOLLENHAUER et al. 2008) }\end{array}$ & Konzentration im Liquor & Reduktion & diagnostisch \\
\hline $\begin{array}{l}\boldsymbol{\beta}^{-}-\text {Amyloid }_{(1-42)} \\
\text { (ALVES et al. 2010; } \\
\text { SIDEROWF et al. 2010) }\end{array}$ & Konzentration im Liquor & Reduktion & prognostisch \\
\hline $\begin{array}{ll}\text { - } & \text { Harnsäure } \\
\text { (SCHWARZSCHILD et al. 2008; } \\
\text { BOGDANOV et al. 2008) }\end{array}$ & Konzentration im Serum & Reduktion & $\begin{array}{l}\text { prognostisch/ } \\
\text { diagnostisch }\end{array}$ \\
\hline $\begin{array}{ll}\text { - } & \text { Transkriptom } \\
\text { (SCHERZER et al. 2007 } \\
\text { GRÜNBLATT et al. 2010) }\end{array}$ & $\begin{array}{l}\text { Transkriptionsprofil der } \\
\text { zellulären } \\
\text { Blutbestandteile }\end{array}$ & $\begin{array}{l}\text { typisches RNS- } \\
\text { Expressionsmuster }\end{array}$ & diagnostisch \\
\hline
\end{tabular}

Bei den Literaturverweisen handelt es sich um Beispiele.

( ${ }^{11}$ C]DTBZ: mit dem Radionuklid Kohlenstoff-11 markiertes Dihydrotetrabenazin; $\left.{ }^{18} \mathrm{~F}\right] \mathrm{Dopa}$ : mit dem Radionuklid Fluor-18 markiertes Dihydroxyphenylalanin; ${ }^{123} \mathrm{IJ}$-CIT: mit dem Radionuklid Jod-123

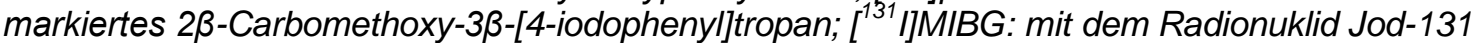
markiertes Metaiodobenzylguanidin; IPS: Idiopathisches Parkinson-Syndrom; PET: PositronenEmissions-Tomographie; REM-Schlaf: Schlafphase mit schnellen Augenbewegungen; RNS: Ribonukleinsäure; SPECT: Einzelphotonen-Emissions-Computertomographie)

Das bildgebende Verfahren der Hirnparenchymsonographie (HPS) ermöglicht es, transkraniell per Ultraschall zerebrale Strukturen darzustellen. Die wichtigste Zielstruktur beim IPS ist die SN. Diese kann durch das temporale Knochenfenster bei axialer Schnittführung beurteilt werden, wobei der Schallkopf präaurikulär platziert wird (WALTER et al. 2004). Die SN zeigt sich hierbei als schwach echogene fleck- oder bandförmige Struktur im schmetterlingsförmigen mesenzephalen Hirnstamm, der von den echoreichen Zisternen umgeben ist.

Charakteristisch bei IPS ist eine Hyperechogenität der SN, die erstmals 1995 von BECKER et al. beschrieben wurde und bei über $90 \%$ der Patienten nachweisbar ist (BERG et al. 2001 a, WALTER et al. 2002). Als hyperechogen wird eine anatomische Struktur dann bezeichnet, wenn ihre echoreiche Fläche vergrößert ist oder wenn das Schallecho eine erhöhte Intensität besitzt (WOITALLA et al. 2010). Als pathologisches Korrelat wird ein erhöhter Eisengehalt in der SN angenommen, wobei das Eisen hier statt an Ferritin an andere Proteine gebunden zu sein scheint (BERG et al. 1999 b). Die Hyperechogenität scheint im Krankheitsverlauf konstant zu bleiben (BERG et al. 2005).

Allerdings können auch gesunde Personen eine Hyperechogenität der SN aufweisen: In einer Studie von BERG et al. (1999 a) mit 301 Freiwilligen ohne extrapyramidale Erkrankungen wurde eine Prävalenz von 8,6 \% ermittelt. Die Hyperechogenität war mit funktionellen Veränderungen im nigrostriatalen System assoziiert, ohne dass die Motorik dieser Personen eingeschränkt gewesen wäre. Eine weitere Studie zeigte, dass über 60jährige Probanden ohne extrapyramidale Erkrankung, aber mit hyperechogener SN eine stärkere motorische Verlangsamung und häufiger Parkinson-typische Symptome wie Rigor oder Tremor aufweisen als vergleichbare Personen ohne Hyperechogenität (BERG et al. 
2001 b). Daraus schlossen die Autoren, die Hyperechogenität würde eine Vulnerabilität des nigrostriatalen Systems für neurodegenerative Einflüsse und damit eine Prädisposition für die Entwicklung eines IPS anzeigen. Mit Hilfe der HPS könnten daher Personen mit einem Risiko für die Entwicklung des IPS identifiziert werden, bevor sich die Erkrankung (motorisch) manifestiert. Diffenzialdiagnostische Bedeutung kommt der HPS zu, weil die SN-Echogenität bei den atypischen Parkinson-Syndromen MSA und progressive supranukleäre Blickparese (PSP) überwiegend normal ist (WALTER et al. 2003).

Das NMS Geruchsstörung bei IPS-Patienten wurde erstmals 1975 von ANSARI beschrieben. Die Angaben zur Prävalenz einer Dysfunktionen des olfaktorischen Systems reichen von 45 \% (ANSARI 1975) über 74 \% (HAWKES et al. 1997) bis zu 97 \% (HAEHNER et al. 2009 a) oder sogar 100 \% (MÜLLER et al. 2002), wobei sich die genannten Studien in ihrer Methodik und Definition unterschieden. Im Vergleich dazu liegt die Häufigkeit in der älteren Allgemeinbevölkerung bei ca. 25 \% (MURPHY et al. 2002). Es können sowohl die Geruchsschwelle als auch die Fähigkeit zur Diskrimination oder Identifikation von Gerüchen beeinträchtigt sein (DAUM et al. 2000, TISSINGH et al. 2001). Betroffen sind Patienten mit fortgeschrittener Erkrankung genauso wie solche mit frühem unbehandelten IPS (DOTY et al. 1992, TISSINGH et al. 2001). Es wird angenommen, dass die Riechstörungen bei IPS der motorischen Manifestation vorausgehen. In zwei Untersuchungen von BERENDSE et al. (2001) und PONSEN et al. (2004) mit erstgradigen Verwandten von Parkinson-Patienten hatten nur Personen mit idiopathischer Hyposmie Zeichen einer subklinischen Dysfunktion des dopaminergen Systems in der Funktionsbildung oder entwickelten ein klinisches IPS. WEBSTER ROSS et al. (2008) wiesen in einer prospektiven populationsbasierten Studie eine Assoziation zwischen eingeschränkter Geruchsidentifikation und zukünftiger Entwicklung eines IPS nach. Riechtests könnten daher bei der Frühdiagnose des IPS sogar im prämotorischen Stadium helfen.

In mehreren Studien wurde festgestellt, die Geruchsfunktionen seien unabhängig von der Krankheitsdauer und dem Erkrankungsstadium bzw. der Schwere der Symptomatik (DOTY et al. 1988, MÜLLER et al. 2002) sowie der Parkinson-Therapie (DOTY et al. 1992). Im Widerspruch dazu fanden andere Autoren zumindest für die Diskriminationsfähigkeit Korrelationen zur Erkrankungsdauer (BOESVELDT et al. 2008), dem Stadium nach HOEHN und YAHR (1967; H\&Y-Stadium) und der Unified Parkinson's Disease Rating Scale (UPDRS) (TISSINGH et al. 2001). In welcher Beziehung die Riechfunktion zum Progress des IPS steht, ist also noch unklar.

Schlafstörungen verschiedener Art sind beim IPS häufig (TANDBERG et al. 1998). Die RBD soll eine Frühmanifestation des IPS mit diagnostischer Relevanz darstellen. Sie ist eine 
Parasomnie, die laut der "International Classification of Sleep Disorders" (ICSD) charakterisiert ist durch „intermittent loss of REM sleep electromyographic (EMG) atonia and by the appearance of elaborate motor activity associated with dream mentation" (AMERICAN ACADEMY OF SLEEP MEDICINE 2001, S. 177). Die Minimalkriterien für die Diagnose basieren auf anamnestisch erhobenen Angaben und fordern Extremitäten- oder Körperbewegungen in Zusammenhang mit dem Trauminhalt und zusätzlich eines der folgenden Symptome: (potenziell) gefährdendes Schlafverhalten, scheinbares Ausagieren von Träumen oder den Schlaf unterbrechendes Schlafverhalten. Objektiviert werden kann die Diagnose durch die Polysomnographie (ebd.). Hierbei stehen die EMG-Ableitungen in der Mental-Region und/ oder an der Extremitätenmuskulatur und die Videobeobachtung des Verhaltens im Vordergrund.

Beim REM-Schlaf ohne Atonie (RWA) liegt eine Erhöhung der Muskelaktivität ohne auffällige motorische Aktivität vor. Möglicherweise ist der RWA eine Vorstufe der RBD (GAGNON et al. 2002). Die Diagnose RWA kann ausschließlich polysomnographisch gestellt werden.

Bis zu $50 \%$ der Parkinson-Patienten zeigen eine RBD (EISENSEHR et al. 2001), auch unbehandelte Patienten mit milder bis moderater Erkrankung (WETTER et al. 1999 und 2000). Die RBD geht in 20-50 \% der Fälle (IRANZO et al. 2005, OLSON et al. 2000, SCHENCK et al. 1996) der motorischen Manifestation des IPS um bis zu 13 Jahre voraus.

Patienten mit idiopathischer RBD unterscheiden sich signifikant in Bezug auf potenzielle frühe Marker des IPS wie Geruchsstörungen, aber auch in motorischen Tests und im Vorliegen autonomer Symptome von gesunden Personen (POSTUMA et al. 2009 b). Zudem konnten mit funktionsbildgebenden Verfahren Zeichen einer nigrostriatalen Neurodegeneration nachgewiesen werden, deren Ausmaß zwischen der bei gesunden Kontrollpersonen und bei Parkinson-Patienten lag (EISENSEHR et al. 2000). In zwei Followup-Untersuchungen von 93 bzw. 29 Patienten mit idiopathischer RBD entwickelten $15 \%$ (POSTUMA et al. 2009 a) bzw. 38 \% (SCHENCK et al. 1996) ein IPS.

Die Liquor-Proteine Tau, P-Tau und A $\mathbf{3 4 2}$ stellen potenzielle prognostische Biomarkerkandidaten zur Identifikation von Patienten mit einem Risiko für kognitive Defizite bzw. Demenz bei Parkinson dar. Prospektive Längsschnittstudien hierzu fehlen aber bislang. Demenz ist ein häufiges NMS des IPS: Die Prävalenz liegt in Deutschland bei etwa 30\% (VON REICHMANN et al. 2010). Langzeitbeobachtungen ergaben, dass im Krankheitsverlauf um die $80 \%$ der Parkinson-Patienten dement werden (AARSLAND et al. 2003, HELY et al. 2008). Als pathologisches Korrelat stehen sowohl neokortikale LewyKörper als auch Alzheimer-typische Veränderungen (Amyloid-Plaques und neurofibrilläre Bündel) in der Diskussion, die in neuropathologischen Untersuchungen auch bei ParkinsonPatienten gefunden wurden (JELLINGER et al. 2002, HURTIG et al. 2000). Möglicherweise 
wirken auch beide Pathologien synergistisch (LASHLEY et al. 2008) oder es gibt verschiedene Formen der Parkinson-Demenz mit unterschiedlichem Beginn, Muster und Progress der kognitiven Defizite (HALLIDAY et al. 2008). BURN (2006) postulierte, die Demenz bei Parkinson würde dann einsetzen, wenn eine toxische Schwelle erreicht wäre, unabhängig davon, ob die pathologischen Veränderungen Parkinson- oder Alzheimer-typisch seien. Neueste Forschungsergebnisse deuten allerdings darauf hin, dass weder Alzheimertypische Veränderungen noch die Lewy-Körper selbst für die neurodegenerativen Symptome verantwortlich sind. Stattdessen rückt der Fokus hin zu kleinen präsynaptischen aSynucleinAggregaten. Diese sollen eine synaptische Dysfunktion verursachen, aus der eine Rarefizierung von Synapsen resultiere (SCHULZ-SCHAEFFER 2010).

Charakteristisch für den $\mathrm{M}$. Alzheimer sind eine Erniedrigung der Konzentration vonßA2 und eine Erhöhung von Tau und P-Tau im Liquor cerebrospinalis (BLENNOW 2004).

$A \beta$-Peptide unterschiedlicher Länge entstehen aus dem Amyloid-Precursor-Protein durch proteolytische Enzyme (HAASS und SELKOE 1993). Das Aß42 weist die höchste Tendenz zur Aggregation auf (SNYDER et al. 1994) und ist der Hauptbestandteil der pathologischen extrazellulären senilen Plaques im Gehirn bei M. Alzheimer (IWATSUBO et al. 1994). Tau ist ein axonal lokalisiertes Mikrotubulus-assoziiertes Protein (BINDER et al. 1985), dessen Konzentration im Liquor einen Marker für das Ausmaß neuronaler Degeneration darstellt (HESSE et al. 2000). Tau wird bei M. Alzheimer an verschiedenen Positionen abnorm phosphoryliert und bildet in dieser Form die intrazellulären neurofibrillären Bündel (IQBAL et al. 1986, MANDELKOW und MANDELKOW 1994). Das an Threonin-181 hyperphosphorylierte Tau ist nach HESSE et al. (2001) nicht einfach ein Marker für Neuronenverlust, da sich seine Konzentration nach akutem Schlaganfall im Gegensatz zur Gesamtkonzentration von Tau nicht verändert. Es kann Alzheimer-Patienten von Gesunden und Patienten mit verschiedenen anderen neurodegenerativen Erkrankungen oder Demenzformen, besonders der Lewy-Körper-Demenz, abgrenzen (LEWCZUK et al. 2004, HAMPEL et al. 2004).

In einer Studie von MOLLENHAUER et al. (2006) wurden bei dementen Parkinson-Patienten im Vergleich zu nicht-dementen Patienten und Kontrollpersonen signifikant niedrigere LiquorLevel von Aß42 und töhere von Tau gemessen. COMPTA et al. (2009) fanden zusätzlich eine signifikante Erhöhung von P-Tau bei dementen Patienten mit IPS verglichen mit nichtdementen Patienten oder Gesunden. In mehreren Studien wurden zudem mittelgradige Veränderungen von $A \beta 42$, nicht hingegen von Tau und $P$-Tau, auch bei nicht dementen Patienten nachgewiesen (COMPTA et al. 2009, MOLLENHAUER et al. 2006, PARNETTI et al. 2008, SJÖGREN et al. 2000, KANEMARU et al. 2000). Kürzlich wurde von ALVES et al. (2010) eine signifikante Reduktion von Aß42 auch beülifien, unbehandelten Parkinson Patienten entdeckt. Die Konzentrationen von $\mathrm{A} \beta 42$ waren mit der äGbthisleistung 
- EINLEITUNG -

assoziiert. SIDEROWF et al. (2010) zeigten erstmals, dass eine Erniedrigung von Aß42 einen unabhängigen Prädiktor für globalen kognitiven Leistungsabfall und Demenz bei Parkinson-Patienten darstellt. Langzeitdaten fehlen aber auch hier noch.

Das Wissen um das individuelle Risiko eines Patienten für kognitive Einschränkungen hätte Einfluss auf die therapeutische Vorgehensweise. Bei Risikopersonen könnte beispielsweise die Gabe von anticholinergen Medikamenten zur Behandlung von Tremor, Dranginkontinenz oder Sialorrhö vermieden werden, da diese die kognitive Leistungsfähigkeit negativ beeinflussen (CAMPBELL et al. 2009). Zudem lässt sich die Kognition bei dementen IPSPatienten durch Acetylcholinesterase-Inhibitoren verbessern (EMRE et al. 2004).

Obwohl es sich bei der Lumbalpunktion zur Gewinnung des Liquor cerebrospinalis um eine invasive Maßnahme handelt, ist die Rate klinisch signifikanter unerwünschter Ereignisse niedrig und die Akzeptanz sogar bei gesunden Probanden ohne direkten Nutzen von der Untersuchung gut (PESKIND et al. 2005). Dennoch wäre es natürlich erstrebenswert, in Zukunft Marker aus einfacher zu gewinnendem Material, wie z. B. Blut oder Urin, zu identifizieren.

\subsection{Zielsetzungen der (Pilot-)Studie}

Ziel der DeNoPa-Kassel-Studie ist zum einen die Identifikation von Biomarkern zur Frühdiagnose des IPS und von prognostischen Markern zur Vorhersage des Verlaufs oder des Therapieansprechens. Die langfristige klinische Verlaufsbeobachtung von De-novoParkinson-Patienten mit anschließender Autopsie ermöglicht es zum anderen, neue Erkenntnisse über Formen der Erkrankung, Einfluss der Medikation, Komplikationen und die Bedeutung von NMS zu gewinnen.

Ziele der vorliegenden Pilotstudie waren:

1. die Überprüfung der Feasibility von Rekrutierung und Datenerhebung bei der DeNoPaKassel-Studie,

2. die Beobachtung nicht-motorischer Frühsymptome bei unbehandelten ParkinsonPatienten und

3. die Bewertung potenzieller Biomarker für das IPS bezüglich ihrer Fähigkeit zur Differenzierung von De-novo-Parkinson-Patienten und gesunden Kontrollpersonen. 


\section{MATERIAL UND METHODEN}

\subsection{Die DeNoPa-Kassel-Studie}

\subsubsection{Studiendesign}

Die DeNoPa-Kassel-Studie ist eine prospektive Langzeit-Follow-up-Studie der ParacelsusElena-Klinik Kassel zu Biomarkern und NMS des IPS. Das Akronym DeNoPa steht hierbei für „De-novo-Parkinson“. Es sollen innerhalb von zwei Jahren 150 unbehandelte Patienten mit der Verdachtsdiagnose eines IPS und 100 Kontrollpersonen ohne bekannte oder behandelte neurologische Erkrankung eingeschlossen werden. Die Kontrollgruppe wird nach Alter, Geschlecht und Bildungsniveau (operationalisiert durch die Dauer des Schulbesuchs in Jahren) gematcht. Studienbeginn war im September 2009. Patienten und Kontrollpersonen werden nach Einschluss in die Studie und im Verlauf alle zwei Jahre umfassend und standardisiert stationär untersucht (Längsschnitt). Alle durchgeführten Untersuchungen sind in Tabelle 2-1 zusammengestellt. Für die Durchführung jeder Untersuchung gibt es einen Hauptverantwortlichen und einen Vertreter. Die Zuordnung der Studienteilnehmer zu den Gruppen ist den Untersuchern bekannt. Eine Verblindung erfolgt nur für die Laboranalyse von Blut und Urin sowie für die Befundung der MRT-Untersuchungen. Die Studie wird 15 bis 20 Jahre dauern. Im Falle des Versterbens der teilnehmenden Patienten ist eine Autopsie zur neuropathologischen Sicherung der Diagnose geplant. Die Studie wurde von der EthikKommission der Landesärztekammer Hessen im März 2009 genehmigt.

\subsubsection{Ein- und Ausschlusskriterien}

Für die Patienten gelten die folgenden Einschlusskriterien:

(1) Verdachtsdiagnose eines IPS (mindestens zwei der drei Symptome Rigor, asymmetrischer Ruhetremor und Bradykinese),

(2) keine dopaminerge Behandlung oder vierwöchige Medikamentenpause vor der stationären Aufnahme,

(3) kein frühzeitiger Verdacht auf ein atypisches Parkinsonsyndrom (häufige Stürze, Blickparese, frühe Demenz, frühe autonome Dysfunktion),

(4) keine bekannte vaskuläre Enzephalopathie (oder andere morphologische Veränderungen in der MRT).

Kontrollpersonen dürfen vor Einschluss keine bekannte bzw. behandelte neurologische Erkrankung haben. 


\section{Tabelle 2-1: Im Rahmen der DeNoPa-Kassel-Studie durchgeführte Untersuchungen}

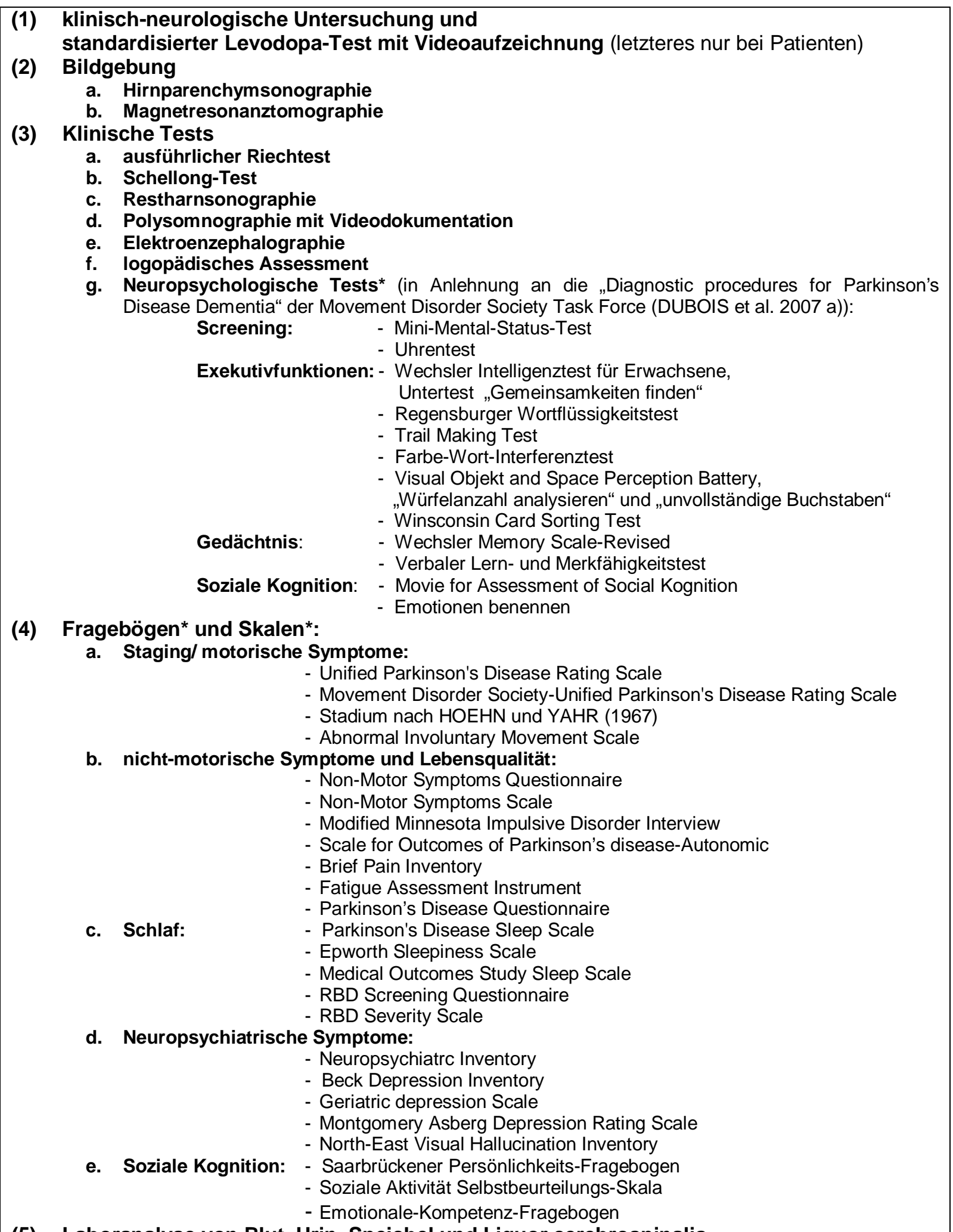

(5) Laboranalyse von Blut, Urin, Speichel und Liquor cerebrospinalis

* Für die Verwendung der neuropsychologischen Tests, Fragebögen und Skalen wurde das Einverständnis der Erstbeschreiber eingeholt. Bei Fehlen einer offiziellen deutschen Version wurde eine Übersetzung ins Deutsche erstellt und diese durch Rückübersetzung validiert.

(RBD: REM-Schlaf-Verhaltensstörung [REM-Schlaf: Schlafphase mit schnellen Augenbewegungen]) 
Alle Studienteilnehmer müssen zwischen 40 und 85 Jahren alt sein und aktuell nicht mehr als 1,5 Autostunden von der Paracelsus-Elena-Klinik entfernt wohnen. Personen mit Kontraindikationen für eine MRT-Untersuchung (z. B. Herzschrittmacher, Phobie) und eine Lumbalpunktion (z. B. erhöhter Hirndruck, Marcumartherapie) werden nicht eingeschlossen.

\subsubsection{Rekrutierung}

Die Rekrutierungsphase läuft von September 2009 bis Januar 2012. Im Anschluss beginnen die ersten Folgeuntersuchungen. Die Rekrutierung der Patienten erfolgt in Kooperation mit niedergelassenen Neurologen vor allem über die Ambulanz, seltener aus dem stationären Bereich der Paracelsus-Elena-Klinik. Die geplanten Rekrutierungszahlen orientieren sich an den Zahlen von De-novo-Patienten, die sich in den letzten Jahren in der Ambulanz vorstellten. Die Kontrollpersonen wurden vor allem durch eine Zeitungsannonce in der „Hessischen/ Niedersächsischen Allgemeinen Zeitung“ (HNA) am 18. März 2009 und über direktes Ansprechen von Angehörigen der Patienten für die Teilnahme an der Studie gewonnen. Es handelt sich also bei beiden Gruppen um „convenience sample“. Es wurde keine Powerkalkulation durchgeführt.

\subsection{Die Pilotstudie zur DeNoPa-Kassel-Studie}

In der hier vorliegenden Pilotstudie wurde eine Auswahl der in der Einschlussuntersuchung erhobenen Daten der bis Ende März 2010 rekrutierten De-novo-Patienten und gesunden Kontrollpersonen ausgewertet. Bei den Patienten wurde der Verdacht auf IPS anhand der Diagnosekriterien der UKPDSBB überprüft. Die UPDRS und die H\&Y-Stadien-Einteilung fanden zur Charakterisierung der beiden Studiengruppen Anwendung. Der NMSQuest und die NMSScale dienten zum Screening eines breiten Spektrums von NMS des IPS. Zur Erkennung von Geruchsstörungen, RBD, OH oder kognitiven Defiziten wurden Symptombezogene Untersuchungen (Riechtest, Polysomnographie, RBD Screening Questionnaire (RBDSQ), Schellongtest und neuropsychologische Tests) durchgeführt, da beispielsweise Einschränkungen des Geruchs oft subjektiv nicht wahrgenommen oder unterschätzt werden (DAUM et al. 2000) und die $\mathrm{OH}$ bei De-novo-Patienten meistens asymptomatisch ist (BONUCCELLI et al. 2003). Der Einfluss der Erkrankung auf die Lebensqualität der Patienten wurde mit Hilfe des Parkinson's Disease Questionnaires (PDQ-39) gemessen. Riechtest und Polysomnographie dienten gleichzeitig zur Evaluierung potenzieller Biomarker. Hinzu kamen die HPS und die Liquordiagnostik. 
Die Untersuchung der Feasibility der DeNoPa-Kassel-Studie beinhaltete die folgenden Aspekte von Rekrutierung und Datenerhebung:

(1) die monatlichen Rekrutierungszahlen von September 2009 bis März 2010,

(2) eine Hochrechnung der Rekrutierungszahlen bis zum Ende des Rekrutierungszeitraums,

(3) den Anteil von Patienten mit der Verdachtsdiagnose IPS unter allen bis März 2010 untersuchten Patienten,

(4) den Anteil der vollständig durchgeführten und erfassten Untersuchungen.

Die Mitarbeit der Autorin der vorliegenden Dissertationsarbeit an der DeNoPa-Kassel-Studie umfasste die Aufklärung von Patienten und Kontrollpersonen und die Einholung und Dokumentation ihres Einverständnisses zur Teilnahme in Zusammenarbeit mit der Studienleiterin Prof. Dr. B. Mollenhauer und der Studienkoordinatorin T. Wicke. Die Dissertantin erhob zudem eine standardisierte Anamnese der Studienteilnehmer inkl. UPDRS und Movement Disorder Society-UPDRS (exkl. des motorischen Teils) sowie die Fragebögen zu nicht-motorischen Symptomen. Sie übernahm des Weiteren bei den Kontrollpersonen der Pilotstudie die ausführliche neuropsychologische Untersuchung (vgl. Tabelle 2-1) und die Schellong-Tests. Darüber hinaus wirkte die Autorin bei der Videoaufzeichnung des L-Dopa-Tests bei den Patienten mit und führte ein logopädisches Assessment (Sprachaufnahmen) durch. In Vertretung der Studienkoordinatorin terminierte die Dissertantin hausinterne und externe Untersuchungen und hatte Telefonkontakt mit Studienteilnehmern und -interessenten. Bei den 7. Kasseler Gesundheitstagen 2010 informierte sie Besucher über die DeNoPa-Kassel-Studie und interessierte sie für die Teilnahme. Außerdem erstellte die Dissertantin eine Datenbank mit allen erhobenen Daten der bis Ende März 2010 rekrutierten Studienteilnehmer und übernahm die statistische Auswertung der Daten für die Pilotstudie.

\subsection{Demographische Daten}

Im Rahmen der Einschluss-Untersuchung wurde mit allen Studienteilnehmern ein standardisiertes Interview durchgeführt, in dem unter anderem demographische und biographische Daten und die Krankheitsgeschichte erfragt wurden. Nach Alter, Geschlecht und Dauer des Schulbesuchs wurden die beiden Studiengruppen gematcht.

\subsection{Neurologische Untersuchung}

Die klinisch-neurologische Untersuchung der Kontrollpersonen erfolgte am Aufnahmetag, die der Patienten im Rahmen des L-Dopa- (Levodopa-)Tests am vierten stationären Tag. Für 
den Test bekamen die seit mindestens zehn Stunden nüchternen Patienten $250 \mathrm{mg}$ Madopar $^{\circledR}$ LT (Levodopa + Benserazid). Vor und eine Stunde nach der Medikamenteneinnahme wurden die Untersuchungen nach Abschnitt III der UPDRS durchgeführt und per Videodokumentation festgehalten. Ab einer Verbesserung des Scores um mindestens $30 \%$ wurde der Test als positiv gewertet (MERELLO et al. 2002). Zur Bestätigung des Verdachts auf IPS wurden die Diagnosekriterien der UKPDSBB (siehe Tabelle 1-2) verwendet. In diesem Zusammenhang wurden auch anamnestische Angaben zur Dauer der Parkinson-Erkrankung eingeholt.

Alle Probanden wurden nach der UPDRS (FAHN et al. 1987) und der Stadieneinteilung von HOEHN und YAHR (1967) klassifiziert. Dies sind wichtige Instrumente zur Beurteilung des klinischen Schweregrades der Parkinson-Krankheit und zur Dokumentation des Krankheitsverlaufs. Die UPDRS (siehe Abbildung 6-1) ist die am weitesten verbreitete Skala zur Messung der Behinderung und Beeinträchtigung von Parkinson-Patienten. Sie ist einfach zu gebrauchen, erfasst diverse Aspekte der Krankheit und findet deshalb sowohl in der klinischen Praxis als auch für wissenschaftliche Zwecke international Anwendung (MOVEMENT DISORDER SOCIETY TASK FORCE ON RATING SCALES FOR PARKINSON'S DISEASE 2003). Die UPDRS ist unterteilt in vier Abschnitte (I: Kognitive Funktionen, Verhalten und Stimmung; II: Aktivitäten des täglichen Lebens; III: motorische Untersuchung; IV: Komplikationen der Behandlung). Die Einordnung bei Teil I, II und IV erfolgt nach Befragung (Interview), Teil III ergibt sich aus dem erhobenen Untersuchungsbefund. Für den Gruppenvergleich wurden abschnittweise Summen aus den für die einzelnen Items vergebenen Punkten gebildet, sodass für Teil I maximal 16, für Teil II 52, für Teil III 108 und für Teil IV 23 Punkte vergeben werden konnten.

Die H\&Y-Stadien berücksichtigen den Grad der Behinderung sowie Störungen von Bewegungen, Gleichgewicht und Gang. NMS gehen nicht in die Beurteilung ein. Die ursprüngliche Skala von 1967 umfasste die Stadien 1 bis 5. Später wurde sie um die Stadien 0, 1,5 und 2,5 erweitert (GOETZ et al. 2004):

Stadium $0 \quad$ Keine Anzeichen der Erkrankung.

Stadium 1 Einseitige Erkrankung.

Stadium 1.5 Einseitige Erkrankung mit axialer Beteiligung.

Stadium 2 Beidseitige Erkrankung ohne Gleichgewichtsstörung.

Stadium 2.5 Leichte beidseitige Erkrankung mit Ausgleich beim Zugtest.

Stadium 3 Leichte bis mäßige beidseitige Erkrankung, leichte Haltungsinstabilität; körperlich unabhängig.

Stadium $4 \quad$ Starke Behinderung; kann noch ohne Hilfe laufen oder stehen.

Stadium 5 Ohne Hilfe an den Rollstuhl gefesselt oder bettlägerig. 


\subsection{Hirnparenchymsonographie}

Für die HPS wurde wie von SCHWEITZER et al. (2006) das Ultraschallgerät ACUSON Antares $^{\mathrm{TM}}$ (Siemens, Erlangen) verwendet. Die Untersuchung erfolgte in Analogie zu BERG et al. (1999 a) durch das präaurikuläre Knochenfenster. Zielparameter waren die Flächen der rechten und linken $\mathrm{SN}$. Sie wurden $a b \geq 0,2 \mathrm{~cm}^{2}$ als hyperechogen gewertet, darunter als normoechogen (SCHWEITZER et al. 2006, MEHNERT et al. 2010).

\subsection{Klinische Tests}

\subsubsection{Riechtest}

Mittels standardisierter „Sniffin“ Sticks“ (Firma Burghart Medizintechnik GmbH, Wedel) wurde die Wahrnehmungsschwelle, die Diskriminations- und die Identifikationsfähigkeit von Gerüchen (erweiterter Test) entsprechend der Bedienungsanleitung untersucht. Der Test wurde nicht seitengetrennt vorgenommen und ist in Tabelle 2-2 beschrieben. Es handelt sich um eine psychophysische Messung, d. h. die subjektive Wahrnehmung von Riechproben wird untersucht. Der Test wurde 1997 von HUMMEL et al. entwickelt. Höhere Ergebnisse repräsentierten jeweils eine bessere Leistung.

Durch Summation der Ergebnisse der drei Subtests für Geruchsschwelle (engl. odor threshold), Diskrimination und Identifikation wurde der TDI-Score berechnet. Dieser wurde von KOBAL et al. (2000) eingeführt und normiert. Auf Grundlage des Scores kann die Riechfunktion in fünf Kategorien eingeteilt werden:

- Normosmie (TDI-Score $>30$ )

- Hyposmie

o mild $(25<$ TDI-Score $\leq 30)$

o moderat $(20<$ TDI-Score $\leq 25)$

o schwer $(15<$ TDI-Score $\leq 20)$

- $\quad$ Anosmie (TDI-Score $\leq 15)$.

\subsubsection{Schellongtest}

Die Durchführung des Schellongtests zum Nachweis einer orthostatischen Dysregulation, benannt nach dem deutschen Internisten Fritz Schellong (1891-1953), erfolgte wie von TIEDT 1985 beschrieben. 
Tabelle 2-2: Aufgabenstellung beim Riechtest mit „Sniffin“ Sticks“

\begin{tabular}{|l|l|}
\hline Subtest & Aufgabe \\
\hline (1) Schwellentest & $\begin{array}{l}\text { Auswahl des riechenden Stifts (Riechstoff: n-Butanol in } \\
\text { unterschiedlichen Konzentrationen) aus drei angebotenen Stiften } \\
\text { (zwei mit geruchsfreiem Lösungsmittel); } \\
\text { Schwellenwerte: 0-16 }\end{array}$ \\
\hline (2) Diskriminationstest & $\begin{array}{l}\text { Auswahl des Stifts mit abweichendem Geruch von drei Stiften; } \\
\text { Ergebnisse: 0-16 Punkte }\end{array}$ \\
\hline (3) Identifikationstest & $\begin{array}{l}\text { Benennen des Duftstoffs im Stift anhand einer Multiple-Choice- } \\
\text { Vorlage; } \\
\text { Ergebnisse: 0-16 Punkte }\end{array}$ \\
\hline
\end{tabular}

Basierend auf einer Konsenserklärung der American Autonomic Society und der American Academy of Neurology von 1996 wurde der Test als pathologisch im Sinne einer orthostatischen Hypotension gewertet, wenn sich innerhalb von drei Minuten nach dem Aufstehen der systolische Blutdruck um mindestens $20 \mathrm{mmHg}$ oder der diastolische Blutdruck um mindestens $10 \mathrm{mmHg}$ reduzierte (THE CONSENSUS COMMITTEE OF THE AMERICAN AUTONOMIC SOCIETY AND THE AMERICAN ACADEMY OF NEUROLOGY 1996). Es wurde jeweils die niedrigste der vier Messungen nach dem Aufstehen (sofort nach dem Aufstehen sowie nach einer, zwei und drei Minuten Stehzeit) mit der vor dem Aufstehen verglichen.

\subsubsection{Polysomnographie}

Alle Studienteilnehmer wurden für zwei Nächte im Schlaflabor der Paracelsus-Elena-Klinik untersucht.

Die durchgeführten Ableitungen und aufgezeichneten Körperfunktionen waren:

- Elektroenzephalographie (EEG; Hirnströme)

- Elektrookulographie (Augenbewegungen)

- Sauerstoffsättigung

- Videoaufzeichnung (Körperposition, Nachtschlafverhalten)

- Registrierung von Schlaf-assoziierten Geräuschen

- Elektromyographie an Bein (Musculus tibialis anterior, Beinbewegungen) und Kinn (Muskelaktivität insbesondere im REM-Schlaf)

- Registrierung des Atemflusses vor Nase und Mund sowie der atemassoziierten abdominalen und thorakalen Exkursionen

Die Analyse erfolgte nach den Regeln des "Manual for the Scoring of Sleep and Associated Events" (IBER et al. 2007) sowie der revidierten Fassung der ICSD (AMERICAN ACADEMY OF SLEEP MEDICINE 2001) und immer durch dieselbe Person.

Ausgewertet wurden die Angaben aus dem Polysomnographie-Befund zum Auftreten eines RWA oder einer RBD in mindestens einer der beiden Nächte. Bei Vorhandensein einer RBD 
wurde zusätzlich der maximal erreichte RBD-Schweregrad (entsprechend der REM Sleep Behavior Disorder Severity Scale, SIXEL-DORING et al. 2009, siehe Tabelle 2-3) in die Analyse aufgenommen.

Tabelle 2-3: REM Sleep Behavior Disorder Severity Scale (SIXEL-DORING et al. 2009, S. S395)

\begin{tabular}{|l|l|}
\hline \multicolumn{2}{|l|}{ motorische Aktivität } \\
\hline $\mathbf{0 .}$ & keine Bewegungen sichtbar (nur REM-Schlaf ohne Atonie) \\
\hline $\mathbf{1 .}$ & $\begin{array}{l}\text { Bewegungen ausschließlich in den distalen Extremitäten oder des Gesichts (Bewegungen } \\
\text { einer Hand oder eines Fußes, Zuckungen des Gesichts) }\end{array}$ \\
\hline $\mathbf{2 .}$ & Bewegungen der proximalen Extremitäten \\
\hline $\mathbf{3 .}$ & axiale Beteiligung \\
\hline Vokalisationen \\
\hline $\mathbf{0}$ & fehlend (Atemgeräusche wie auch Schnarchen können vorhanden sein) \\
\hline $\mathbf{. 1}$ & vorhanden (z. B. Reden, Lachen, Rufen, Schreien) \\
\hline
\end{tabular}

Die Einstufung von motorischen Ereignissen während des REM-Schlafs (Schlafphase mit schnellen Augenbewegungen) basiert auf der Polysomnographie mit synchronisiertem Video. Der Schweregrad entspricht dem höchsten Score, der während der Untersuchung vergeben wurde.

\subsubsection{Neuropsychologische Tests}

Ausgewertet wurden die neuropsychologischen Screeningtests zu kognitiven Leistungen: der Mini-Mental-Status-Test (MMST) (FOLSTEIN 1975, deutschsprachige Fassung von KESSLER et al. 1990; siehe Abbildung 6-2) und der Uhrentest (SHULMAN KI et al. 1986). Die Auswertung des Uhrentests erfolgte nach SHULMAN KI et al. (1993) auf einer Skala von 1 („perfekt") bis 6 (keinerlei Darstellung einer Uhr; siehe Abbildung 6-3). Beim MMST spricht ein Score von < 26 von 30 Punkten (DUBOIS et al. 2007 a), beim Uhrentest ein Score von $\geq 3$ Punkten (BRODATY und MOORE 1997) für eine kognitive Beeinträchtigung.

(Auf Grund des kleinen Stichprobenumfangs wurde zum jetzigen Zeitpunkt auf eine detaillierte Analyse der Tests für Exekutivfunktionen, Gedächtnis und soziale Kognition verzichtet.)

\subsection{Fragebögen}

Die Fragebögen wurden zum Teil in Interviewform während des Aufnahmegesprächs und zum Teil während des stationären Aufenthalts selbstständig durch die Studienteilnehmer bearbeitet.

$\mathrm{Zu}$ ersteren gehörten die interkulturell adaptierten Versionen des Non-Motor Symptoms Questionnaires (NMSQuest; CHAUDHURI et al. 2006 b, MARTINEZ-MARTIN et al. 2007; siehe Abbildung 6-4) und der Non-Motor Symptoms Scale (NMSScale; CHAUDHURI et al. 2007; siehe Abbildung 6-5) in deutscher Sprache (STORCH et al. 2010). Fragebogen und 
Skala beziehen sich auf Symptome, die während des letzten Monats aufgetreten sind. Im NMSQuest geht es um das Vorkommen derselben (,ja“/ „nein“), während in der NMSScale quantitativ die Schwere $(0=$,keine Belastung/ Beeinträchtigung" bis $3=$ „erhebliche Belastung“) und Häufigkeit (1= „selten“ bis 4= „sehr häufig“) erfasst wird. Beim Fragebogen wird der prozentuale Anteil von Ja-Antworten berechnet (maximal 30/30 = $100 \%)$. Bei der Skala werden die Wertungen für Schwere und Häufigkeit multipliziert und die Ergebnisse zu einem Gesamtwert addiert (Maximalpunktzahl: 360).

Die Fragebögen zum Selbstausfüllen für die Patienten waren der Parkinson's Disease Questionnaire (PDQ-39; PETO et al. 1995, BERGER et al. 1999; siehe Abbildung 6-6) und der RBD Screening Questionnaire (RBDSQ; STIASNY-KOLSTER et al. 2007; siehe Abbildung 6-7).

Der PDQ-39 dient zur Messung des Einflusses der Parkinson-Krankheit auf die aktuelle Lebenssituation. Er umfasst die Beeinträchtigungen bei alltäglichen Aktivitäten und Mobilität, das emotionale Befinden, die soziale Unterstützung, die Stigmatisierung, Kommunikationund Kognitionsaspekte und das körperliche Befinden (PETO et al. 1995). Gefragt wird, ob Beeinträchtigungen „niemals“ (1 Punkt), „selten“, „manchmal“, „häufig“ oder „immer“ (5 Punkte) auftreten. Durch Summierung der Punkte und Standardisierung auf einer Skala von 1 bis 100 wird der Summary Index (PDQ-39SI) berechnet, der einen allgemeinen Eindruck der krankheitsbezogenen Lebensqualität bietet (PETO et al. 1998). Niedrige Werte repräsentieren eine bessere, hohe Werte eine schlechtere Lebensqualität. Die deutsche Version des Fragebogens wurde 1999 von BERGER et al. erstellt und validiert.

Der RBDSQ ist ein Fragebogen zur Selbstbeurteilung des eigenen Schlafverhaltens durch die Patienten. In Ja/nein-Fragen werden insbesondere das Träumen, nächtliches Agieren und Vokalisieren, Störungen des Schlafs sowie das Vorhandensein einer neurologischen Erkrankung abgefragt. Der RBDSQ-Score berechnet sich aus der Anzahl der Ja-Antworten. $A b \geq 5$ Punkten von 13 wird der Test als positiv gewertet, ein Hinweis auf klinische Symptome der RBD (STIASNY-KOLSTER et al. 2007).

Unvollständig ausgefüllte Fragebögen wurden nicht in die Auswertung einbezogen.

\subsection{Liquordiagnostik}

Die Gewinnung von Liquor cerebrospinalis durch Lumbalpunktion erfolgte bei Patienten am dritten, bei Kontrollpersonen am zweiten stationären Tag nach Ausschluss von Kontraindikationen wie erhöhtem Hirndruck oder Gerinnungsstörungen durch MRT und Gerinnungsuntersuchungen am Vortag. Alle Probanden waren nüchtern und die Patienten unbehandelt. 
Im gewonnenen Liquor cerebrospinalis wurden neben der Basisdiagnostik (Zellzählung, Gesamteiweiß, Immunglobulin- und Albumin-Konzentrationen und -quotienten) die Demenzbzw. Destruktionsmarker Tau, P-Tau (an Threonin-181 hyperphosphoryliert) und Aß42 quantifiziert. Hierfür wurden die kommerziell erhältlichen enzymgekoppelten Immunabsorptionsassays (ELISA; engl. enzyme linked immunosorbent assays) INNOTEST ${ }^{\circledR}$ hTau Ag, INNOTEST ${ }^{\circledR}$ PHOSPHO-TAU $_{(181 P)}$ und INNOTEST® $\beta$-AMYLOID $(1-42)$ der Firma Innogenetics, Gent, Belgien, verwendet. Diese Analysen erfolgten im neurochemischen Labor der Universitätsmedizin Göttingen.

\subsection{Statistische Analyse}

Die Auswertung und Aufbereitung der Daten erfolgte mit SPSS ${ }^{\circledR}$, Version 17.0, IBM ${ }^{\circledR}$ SPSS $^{\circledR}$ Statistics, Version 19, Microsoft Excel ${ }^{\circledR}$ und Word $^{\circledR} 2007$.

Im Rahmen der deskriptiven Auswertung wurden für quantitative Daten die statistischen Kenngrößen Mittelwert, Standardabweichung, Median, Minimum und Maximum berechnet, um die Verteilung relevanter Merkmale innerhalb der beiden Studiengruppen zu beschreiben. Zur Visualisierung der Verteilung der Daten innerhalb bzw. zwischen den Gruppen wurden gegebenenfalls Boxplots erstellt. Die Lage der wesentlichen Kennwerte im Boxplot wird aus Abbildung 2-1 ersichtlich. Für qualitative wie für quantitative Daten wurden die Häufigkeiten der verschiedenen Merkmalsausprägungen bestimmt.

Zum Gruppenvergleich zwischen den Parkinson-Patienten und den gesunden Kontrollpersonen kamen Signifikanztests zur Anwendung, speziell der U-Test nach MannWhitney. Bei nominalskalierten Variablen wurden Kreuztabellen erstellt und die Unabhängigkeit der Merkmale bzw. deren Zusammenhang mittels Chi-Quadrat-Tests analysiert. Es wurde eine Signifikanzniveau von $5 \%$ zu Grunde gelegt. Bei p-Werten, d. h. Signifikanzwerten, vo .05 wurde also die Nullhypothese verworfen und die Gegenhypothese angenommen. Die Gegenhypothese geht allgemein davon aus, dass beide Stichproben verschiedenen Grundgesamtheiten entstammen und Unterschiede in Medianen bzw. Mittelwerten nicht zufällig zustande gekommen sind.

Zur Beurteilung der Güte eines Tests wurden gegebenenfalls die Kennwerte Sensitivität und Spezifität berechnet (siehe Abbildung 2-2). Die Sensitivität gibt den Anteil der durch den Test korrekt als positiv klassifizierten Personen an der Gesamtheit der in Wirklichkeit positiven Personen an (Richtig-Positiv-Rate). Die Spezifität entspricht dem Anteil der durch den Test korrekt als negativ klassifizierten Personen an der Gesamtheit der negativen Personen (Richtig-Negativ-Rate). Ein diagnostischer Test hat eine hohe Sensitivität, wenn durch inn (fast) alle Kranken als krank (positives Ergebnis) erkannt und eine hohe Spezifität, wenn (fast) alle Gesunden als gesund (negatives Ergebnis) eingeschätzt werden. 
* 4 extremer Ausreißer (>3 Kastenlängen außerhalb der Box)

- 4 milder Ausreißer (1,5 bis 3 Kastenlängen außerhalb der Box)

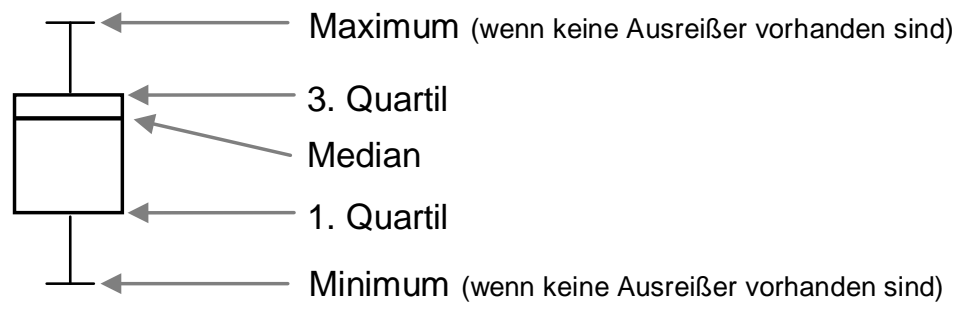

Abbildung 2-1: Lage der Kennwerte einer Verteilung im Boxplot

\begin{tabular}{|ll|l|l|}
\cline { 3 - 4 } \multicolumn{1}{c|}{} & \multicolumn{2}{c|}{ Krankheit } \\
\multirow{2}{*}{ Testergebnis } & vorhanden & nicht vorhanden \\
\cline { 2 - 4 } & nesitiv & richtig positiv (RP) & falsch positiv (FP) \\
\hline
\end{tabular}

$$
\begin{aligned}
& \text { Sensitivität }=\frac{R P}{R P+F P} \\
& \text { Spezifität }=\frac{F N}{F N+R N}
\end{aligned}
$$

Abbildung 2-2: Vierfeldertafel und Gütekriterien eines diagnostischen Tests 


\section{ERGEBNISSE}

Grundlage dieser Pilotstudie sind die Daten der Studienteilnehmer, welche zwischen September 2009 und März 2010 in die DeNoPa-Kassel-Studie eingeschlossen wurden.

\subsection{Demographische Daten}

Im genannten Zeitraum wurden die Daten von dreißig Patienten (15 männlich, 15 weiblich) und zehn Kontrollpersonen (6 männlich, 4 weiblich) erhoben. Das mittlere Alter der Patienten lag bei 66,3 Jahren, das der Kontrollpersonen bei 63,6 Jahren. Die Dauer des Schulbesuchs in Jahren betrug in der Patientengruppe im Mittel 9,4 Jahre, die Kontrollpersonen besuchten im Mittel 10,8 Jahre die Schule. Alle weiteren Kenngrößen der Verteilung von Alter und Bildungsstand in den Studiengruppen sind in Tabelle 3-1 zusammengestellt. Es bestand kein signifikanter Unterschied im Geschlechterverhältnis $(p=.66)$, dem Alter $(p=.23)$ oder dem Bildungsstand $(p=.06)$ der beiden Gruppen.

Tabelle 3-1: Demographische Daten der Patienten- (PG) und Kontrollgruppe (KG)

\begin{tabular}{|c|c|c|c|c|c|c|c|c|}
\hline $\begin{array}{l}\text { Variable } \\
\text { [Einheit] }\end{array}$ & Gruppe & $\mathbf{N}$ & $\mathbf{M}$ & SD & Median & Min. & Max. & p-Wert \\
\hline \multirow{2}{*}{$\begin{array}{l}\text { Alter } \\
\text { [Jahre] }\end{array}$} & PG & 30 & 66,3 & 10,4 & 67,5 & 42,0 & 84,0 & \multirow{2}{*}{.23} \\
\hline & $K G$ & 10 & 63,6 & 5,3 & 63,5 & 55,0 & 71,0 & \\
\hline \multirow{2}{*}{$\begin{array}{l}\text { Dauer des } \\
\text { Schulbesuchs } \\
\text { [Jahre] }\end{array}$} & $P G$ & 30 & 9,4 & 1,7 & 9,5 & 6,5 & 13,0 & \multirow{2}{*}{.06} \\
\hline & $K G$ & 10 & 10,8 & 2,0 & 10,0 & 8,0 & 13,0 & \\
\hline
\end{tabular}

Deskriptive Kennwerte und Ergebnisse des Signifikanztests.

(M: Mittelwert; Max.: Maximum; Min.: Minimum; N: Stichprobenumfang; p-Wert: Signifikanzwert; SD: Standardabweichung)

\subsection{Neurologische Untersuchung}

Den Diagnosekriterien für Parkinson-Syndrome entsprechend waren bei allen Patienten eine Bradykinese und mindestens eines der weiteren Kardinalsymptome vorhanden. Rigor und Ruhetremor zeigten sich jeweils bei über $70 \%$ der Patienten. Eine posturale Instabilität war nur bei $43 \%$ der Patienten vorhanden (siehe Abbildung 3-1). Diese war jedoch in keinem Fall prominent mit Stürzen im ersten Jahr, sodass differenzialdiagnostisch von einer PSP hätte ausgegangen werden müssen. $80 \%$ der Patienten hatten einen einseitigen Beginn und eine persistierende Asymmetrie der Symptome. Bei ebenso vielen Patienten war die Krankheit progredient. 


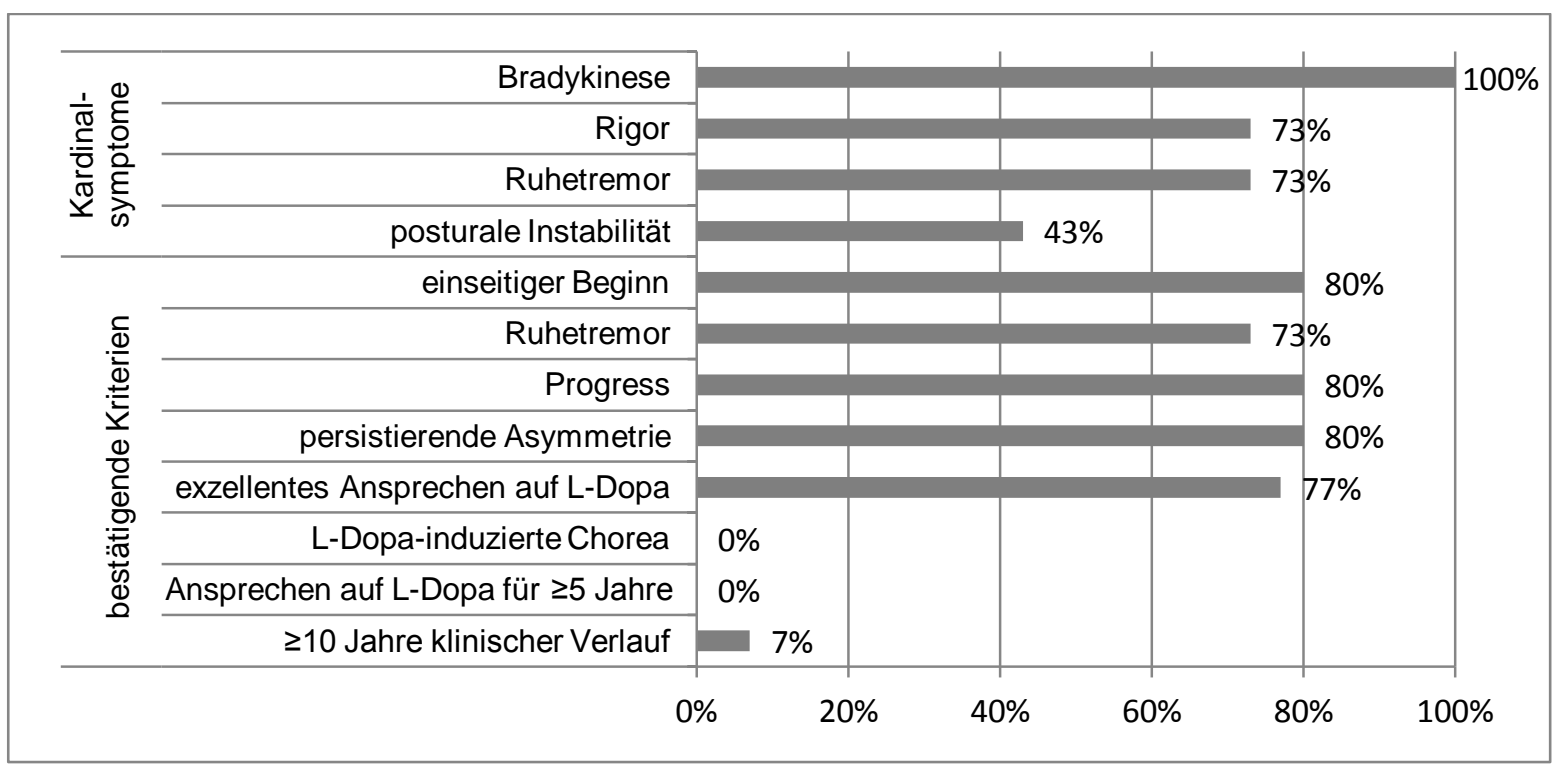

Abbildung 3-1: Häufigkeit der Kardinalsymptome und der bestätigenden Kriterien für das idiopathische Parkinson-Syndrom in der Patientengruppe $(N=30)$ in \% (N: Stichprobenumfang)

Die anamnestisch erhobene mittlere Erkrankungsdauer der Patienten bei Einschluss in die Studie lag bei 32 Monaten (Median: 18,5 Monate), wobei der Zeitraum insgesamt zwischen 0,5 und 120 Monaten variierte (siehe Abbildung 3-2).

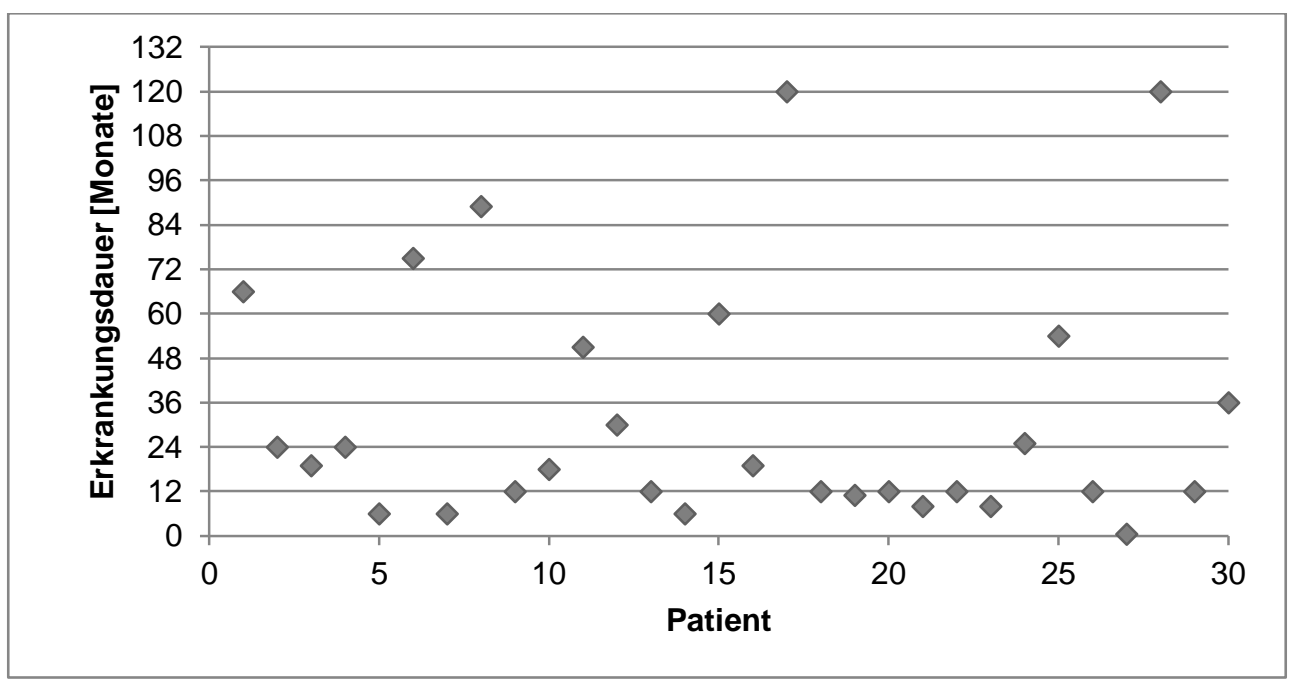

Abbildung 3-2: Erkrankungsdauer der 30 Patienten beim Einschluss in die DeNoPaKassel-Studie in Monaten

Der L-Dopa-Test war bei $23(77 \%)$ der Patienten positiv. Von den sieben Patienten mit negativem Testergebnis zeigten drei Patienten keine L-Dopa-Sensitivität der Symptome. Die maximale Verbesserung des UPDRS-III-Scores durch L-Dopa betrug 72,2\% (siehe Abbildung 3-3). Bei einem der Patienten ohne Ansprechen fiel jedoch ein zweiter Test mit 250 mg Isicom ${ }^{\circledR}$ anstatt Madopar $^{\circledR}$ LT (enthält Carbidopa anstatt Benserazid als L-Dopa- 
Decarboxylasehemmstoff) mit $32 \%$ Besserung positiv aus und bei einem weiteren Patienten, dessen Test nach 60 Minuten noch negativ (15,4\%) war, zeigte sich nach 120 Minuten dann doch eine Besserung um $50 \%$.

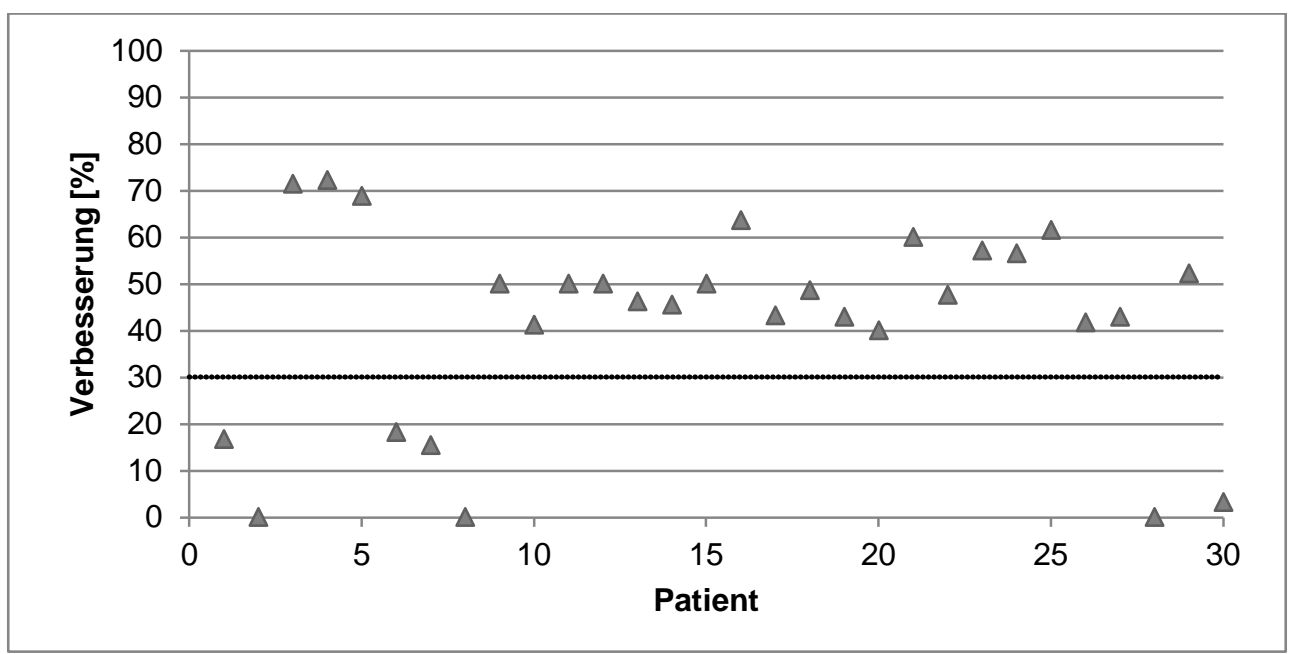

Abbildung 3-3: Prozentuale Verbesserung der Punktzahl im Abschnitt III der Unified Parkinson's Disease Rating Scale bei den 30 Patienten während des Levodopa-Tests. Ab einer Verbesserung von 30 \% (gepunktete Linie) gilt der Test als positiv.

25 (83\%) der Patienten erfüllten drei oder mehr der bestätigenden Kriterien für das IPS (siehe Abbildung 3-4).

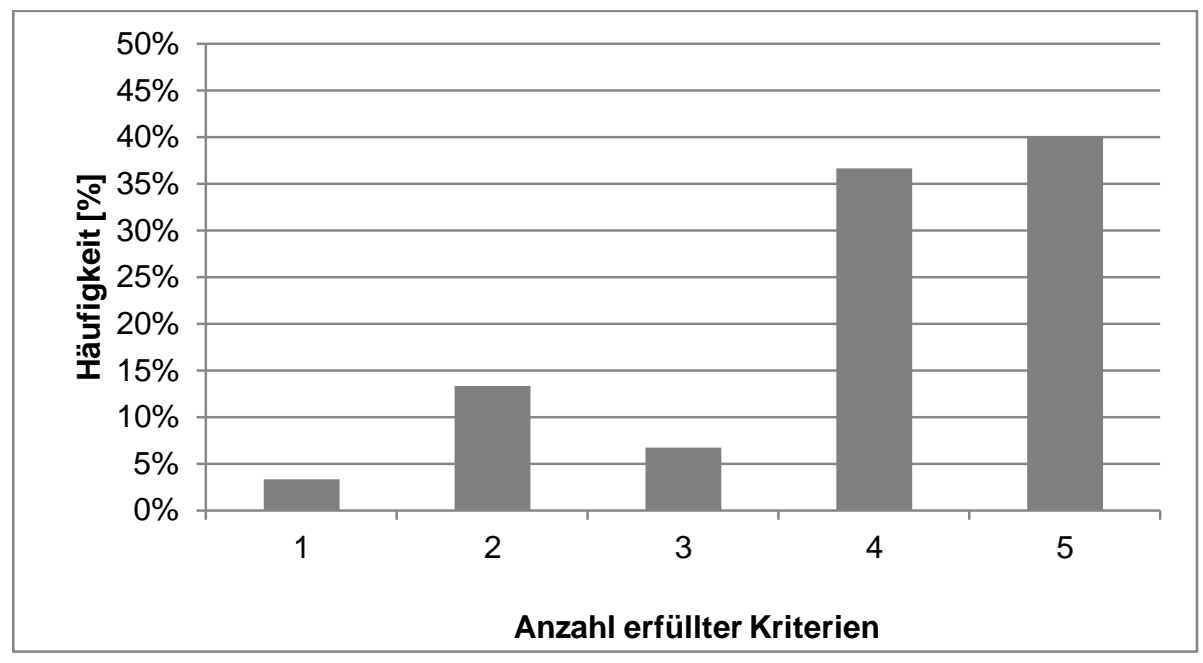

Abbildung 3-4: Anzahl erfüllter bestätigender Kriterien für die klinische Diagnose eines idiopathischen Parkinson-Syndroms entsprechend den Diagnosekriterien der United Kingdom Parkinson's Disease Society Brain Bank (HUGHES et al. 1992) in der Patientengruppe $(\mathrm{N}=30)$

( $N$ : Stichprobenumfang)

Die Ergebnisse der UPDRS und die H\&Y-Stadien in den Studiengruppen sind in Tabelle 3-2 zusammengestellt. 
Tabelle 3-2: Ergebnisse der Unified Parkinson's Disease Rating Scale (UPDRS), Abschnitt I-IV und Stadien nach HOEHN und YAHR (1967; H\&Y-Stadien) in der Patienten- (PG) und Kontrollgruppe (KG)

\begin{tabular}{|c|c|c|c|c|c|c|c|c|}
\hline Variable & Gruppe & $\mathbf{N}$ & $M$ & SD & Median & Min. & Max. & p-Wert \\
\hline \multirow{2}{*}{$\begin{array}{l}\text { UPDRS } \\
\text { Abschnitt I }\end{array}$} & $P G$ & 30 & 2,0 & 2,0 & 2,0 & 0 & 6 & \multirow{2}{*}{.02} \\
\hline & KG & 10 & 0,5 & 0,7 & 0,0 & 0 & 2 & \\
\hline \multirow{2}{*}{$\begin{array}{l}\text { UPDRS } \\
\text { Abschnitt II }\end{array}$} & $P G$ & 30 & 8,9 & 5,1 & 8,0 & 2 & 24 & \multirow{2}{*}{$<.001$} \\
\hline & KG & 10 & 0,3 & 0,9 & 0,0 & 0 & 3 & \\
\hline \multirow{2}{*}{$\begin{array}{l}\text { UPDRS } \\
\text { Abschnitt III }\end{array}$} & $P G$ & 30 & 20,8 & 10,1 & 19,0 & 6 & 41 & \multirow{2}{*}{$<.001$} \\
\hline & $K G$ & 10 & 0,5 & 0,8 & 0,0 & 0 & 2 & \\
\hline \multirow{2}{*}{$\begin{array}{l}\text { UPDRS } \\
\text { Abschnitt IV }\end{array}$} & $P G$ & 30 & 0,0 & 0,0 & 0,0 & 0 & 0 & \multirow{2}{*}{1.00} \\
\hline & KG & 10 & 0,0 & 0,0 & 0,0 & 0 & 0 & \\
\hline \multirow[t]{2}{*}{ H\&Y-Stadien } & $P G$ & 30 & 1,9 & 0,6 & 1,5 & 1 & 3 & \multirow{2}{*}{$<.001$} \\
\hline & $K G$ & 10 & 0,0 & 0,0 & 0,0 & 0 & 0 & \\
\hline
\end{tabular}

Deskriptive Kennwerte und Ergebnisse des Signifikanztests. Signifikante Ergebnisse sind grau hervorgehoben.

(M: Mittelwert; Max.: Maximum; Min.: Minimum; N: Stichprobenumfang; p-Wert: Signifikanzwert; SD: Standardabweichung)

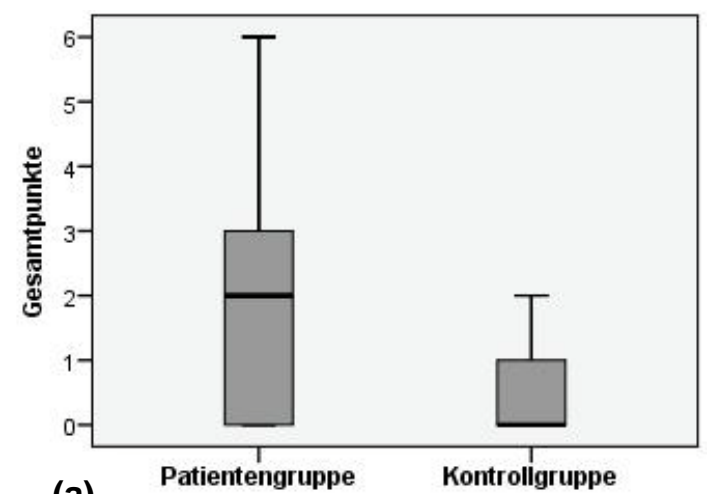

(a)

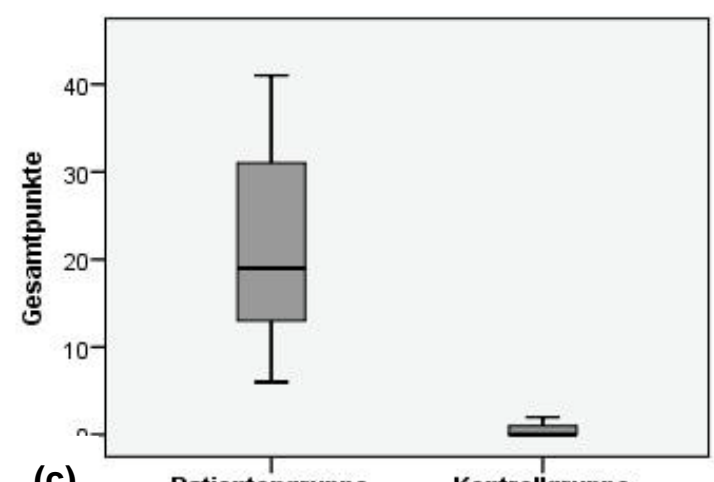

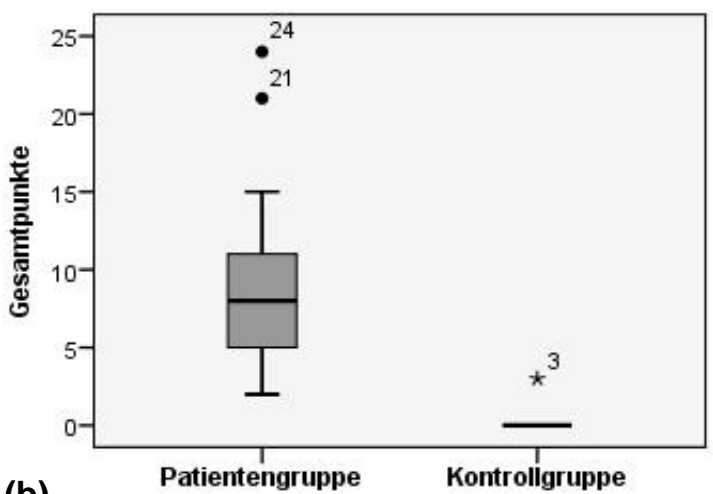

(b) 
In Abschnitt I der UPDRS (siehe Abbildung 3-5a) hatten die Patienten eine mittlere Summe von 2 Punkten, während die Kontrollpersonen im Mittel 0,5 Punkte bekamen. Beide Gruppen unterschieden sich signifikant $(p=.02)$ in diesem Teil des UPDRS.

In Abschnitt II (siehe Abbildung 3-5b) lagen die Ergebnisse der Patienten im Mittel bei 8,9 Punkten. Neun der Kontrollpersonen empfanden keinerlei Einschränkungen im täglichen Leben. Der Mittelwert betrug 0,3 Punkte. Der Unterschied zwischen den Gruppen war signifikant $(p<.001)$. Über zwei Drittel der Patienten bejahten unabhängig vom Ausprägungsgrad Schwierigkeiten beim Schreiben, Gehen, Schneiden von Speisen bzw. Handhaben von Utensilien und Anziehen (siehe Tabelle 3-3). Diese Beeinträchtigungen waren beim überwiegenden Teil der Patienten leichtgradig (geringe Verlangsamung, milde Schwierigkeiten, keine Hilfe nötig). Der Tremor war bei über der Hälfte der Patienten moderat und störend (2 Punkte).

Tabelle 3-3: Beeinträchtigungen bei Aktivitäten des täglichen Lebens mit einer Häufigkeit von über zwei Dritteln in der Patientengruppe $(N=30)$ und deren Ausprägungsgrad in der Unified Parkinson's Disease Rating Scale, Abschnitt II

\begin{tabular}{|l|l|r|r|r|r|r|}
\hline & Beeinträchtigung & Häufigkeit, & \multicolumn{3}{|c|}{ Ausprägungsgrad [\%] } \\
(Frage) & absolut (\%) & $\mathbf{1}$ & $\mathbf{2}$ & $\mathbf{3}$ & $\mathbf{4}$ \\
\hline \multirow{2}{*}{1.} & Handschrift (8) & $25(83)$ & 48 & 32 & 20 & 0 \\
\cline { 2 - 6 } & Tremor (16) & $25(83)$ & 24 & 56 & 20 & 0 \\
\hline 2. & Gehen (15) & $23(77)$ & 91 & 9 & 0 & 0 \\
\hline 3. & Handhabung von Utensilien (9) & $22(73)$ & 73 & 23 & 4 & 0 \\
\hline 4. & Anziehen (19) & $20(67)$ & 90 & 5 & 5 & 0 \\
\hline
\end{tabular}

(N: Stichprobenumfang)

In Abschnitt III (siehe Abbildung 3-5c) erreichten die Patienten im Mittel 20,8 Punkte. In der Kontrollgruppe lag das mittlere Ergebnis bei 0,5 Punkten. Der Gruppenvergleich ergab eine statistische Signifikanz $(p<.001)$.

Keiner der Studienteilnehmer bekam Punkte im Abschnitt IV der UPDRS.

Das mittlere H\&Y-Stadium der Patienten bei Aufnahme betrug 1,9. Die Kontrollpersonen zeigten keine Anzeichen für ein IPS. Dementsprechend hatten alle das H\&Y-Stadium 0. Die Verteilung der Stadien innerhalb der Patientengruppe ist in Abbildung 3-6 dargestellt. 


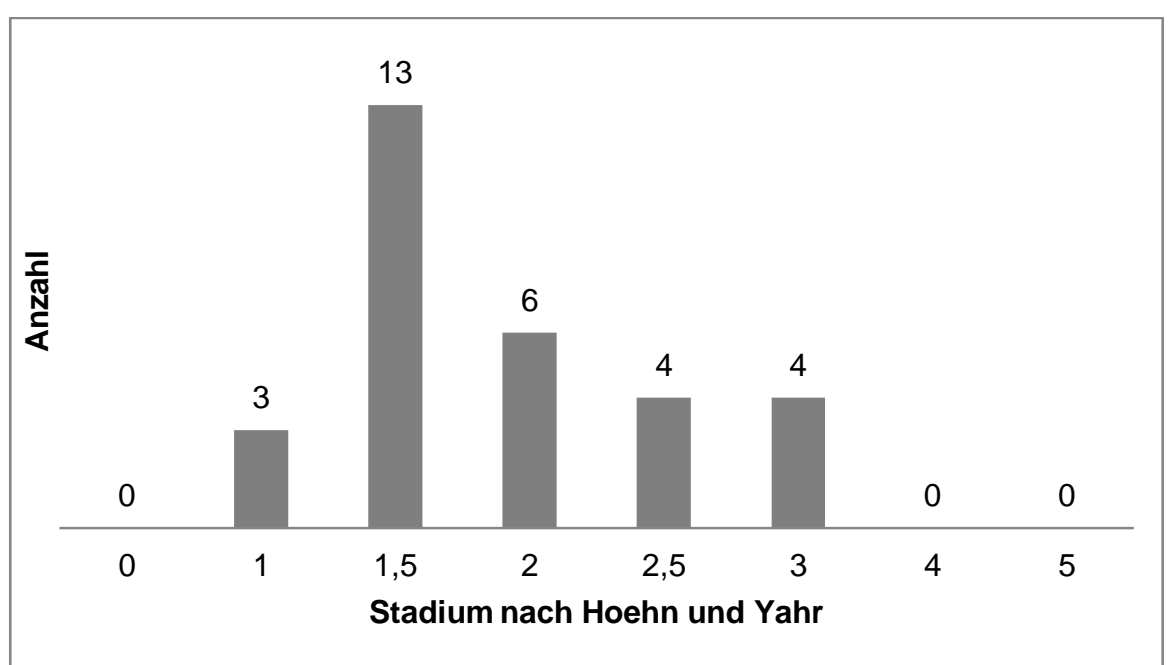

Abbildung 3-6: Häufigkeit der Stadien nach HOEHN und YAHR (1967) in der Patientengruppe

\subsection{Hirnparenchymsonographie}

Die HPS der SN ergab bei den Patienten eine mittlere Fläche von 0,24 $\mathrm{cm}^{2}$ rechts und von $0,25 \mathrm{~cm}^{2}$ links (siehe Abbildung 3-7). Bei zwei Patienten war beidseits und bei zwei links kein ausreichendes Schallfenster vorhanden, sodass der Hirnstamm nicht adäquat dargestellt werden konnte und keine Messung erfolgte. Bei allen Kontrollpersonen konnte die SN bilateral ausgemessen werden. Die Mittelwerte in der Kontrollgruppe lagen bei $0,12 \mathrm{~cm}^{2}$ rechts und bei $0,12 \mathrm{~cm}^{2}$ links (siehe Abbildung 3-7).

Weitere statistische Maßzahlen der Verteilung der Flächen in beiden Gruppen sind in Tabelle 3-4 gegenübergestellt. Es zeigten sich beidseits signifikante Unterschiede $(p<.001)$ in der Echogenität der SN zwischen Patienten- und Kontrollgruppe.

Tabelle 3-4: Ergebnisse der Hirnparenchymsonographie der Substantia nigra (SN) in der Patienten- (PG) und Kontrollgruppe (KG)

\begin{tabular}{|c|c|c|c|c|c|c|c|c|}
\hline $\begin{array}{l}\text { Variable } \\
\text { [Einheit] }\end{array}$ & Gruppe & $\mathbf{N}$ & $\mathbf{M}$ & SD & Median & Min. & Max. & p-Wert \\
\hline \multirow{2}{*}{$\begin{array}{l}\text { Fläche der SN } \\
\text { rechts }\left[\mathrm{cm}^{2}\right]\end{array}$} & $P G$ & 28 & 0,24 & 0,1 & 0,24 & 0,12 & 0,36 & \multirow{2}{*}{$<.001$} \\
\hline & KG & 10 & 0,12 & 0,1 & $\begin{array}{l}0,09 \\
\end{array}$ & 0,04 & 0,27 & \\
\hline \multirow{2}{*}{$\begin{array}{l}\text { Fläche der SN } \\
\text { links }\left[\mathrm{cm}^{2}\right]\end{array}$} & $P G$ & 26 & 0,25 & 0,1 & 0,26 & 0,08 & 0,43 & \multirow{2}{*}{$<.001$} \\
\hline & KG & 10 & 0,12 & 0,1 & 0,10 & 0,04 & 0,22 & \\
\hline
\end{tabular}

Deskriptive Kennwerte und Ergebnisse des Signifikanztests. Signifikante Ergebnisse sind grau hervorgehoben.

(M: Mittelwert; Max.: Maximum; Min.: Minimum; N: Stichprobenumfang; p-Wert: Signifikanzwert; SD: Standardabweichung) 
- ERGEBNISSE -

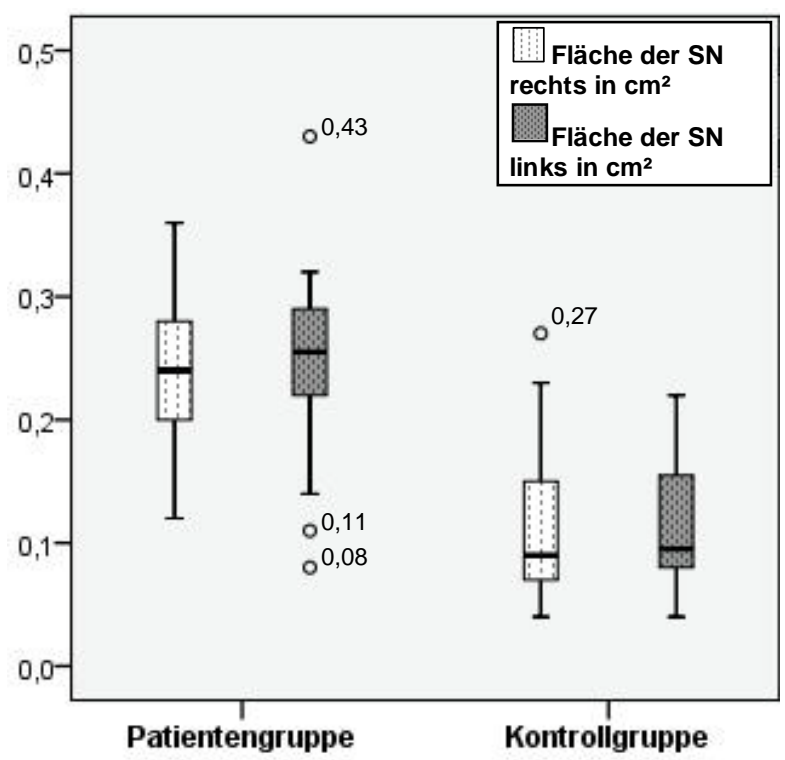

Abbildung 3-7: Boxplots zu den Flächen der rechten und linken Substantia nigra (SN) in der Patienten- $\left(N_{\text {rechts }}=28, N_{\text {links }}=26\right)$ und Kontrollgruppe $(N=10)$

( $N$ : Stichprobenumfang)

Von den 26 Patienten mit beidseitig ausreichendem Schallfenster hatten 22 beidseits eine hyperechogene SN. Bei einem Patienten wurde rechts eine normale Echogenität $\left(0,18 \mathrm{~cm}^{2}\right)$ und links eine erhöhte $\left(2,2 \mathrm{~cm}^{2}\right)$ gemessen. Bei drei Patienten war die SN beidseits normal. Die beiden Patienten mit einseitigem Schallfenster hatten beide an der einsehbaren Seite eine Hyperechogenität. Unter den Kontrollpersonen gab es einen mit beidseits (rechts 0,23, links $0,22 \mathrm{~cm}^{2}$ ) und einen mit einseitig (rechts 0,27, links 0,19 $\mathrm{cm}^{2}$ ) erhöhter Echogenität. Alle anderen hatten eine normale SN-Fläche (siehe Tabelle 3-5).

Tabelle 3-5: Echogenität der Substantia nigra (SN)

\begin{tabular}{|l|c|c|}
\hline & $\begin{array}{c}\text { Patienten } \\
(\mathrm{N}=28)\end{array}$ & $\begin{array}{c}\text { Kontrollpersonen } \\
(\mathrm{N}=10)\end{array}$ \\
\hline $\begin{array}{l}\text { Beidseits hyperechogen } \\
\left(\mathrm{SN} \geq 0,2 \mathrm{~cm}^{2}\right)\end{array}$ & $22(78,6 \%)$ & $1 \quad(10 \%)$ \\
\hline Einseitig hyperechogen & $3(10,7 \%)^{\star}$ & $1(10 \%)$ \\
\hline $\begin{array}{l}\text { Beidseits normoechogen } \\
\left(\mathrm{SN}<0,2 \mathrm{~cm}^{2}\right)\end{array}$ & $3(10,7 \%)$ & $8(80 \%)$ \\
\hline
\end{tabular}

* davon 2 Patienten mit einseitigem Schallfenster

( $N$ : Stichprobenumfang)

Eine Zuordnung der Studienteilnehmer zur Patienten- bzw. Kontrollgruppe anhand der 0,2 cm²-Grenze wäre bei $89 \%$ der Patienten und bei $80 \%$ der Kontrollpersonen korrekt gewesen (wobei auch eine einseitige Hyperechogenität als hinreichendes Kriterium für ein IPS gewertet wurde). 


\subsection{Klinische Tests}

\subsubsection{Riechtest}

Die mittlere psychophysisch gemessene Geruchsschwelle lag in der Patientengruppe bei 5,6, in der Kontrollgruppe bei 8,8. Der mittlere Diskriminationswert der Patienten betrug 9,3, der der Kontrollpersonen 13,3. Der Identifikationstest ergab im Mittel einen Wert von 8,8 in der Patientengruppe und 12,9 in der Kontrollgruppe. Die Verteilung der Ergebnisse innerhalb der Gruppen ist in Abbildung 3-8 und Tabelle 3-6 dargestellt. Bei einem Patienten wurde kein Riechtest durchgeführt und eine Schwellenwertbestimmung wurde auf Wunsch der Kontrollperson abgebrochen.

Die Patienten schnitten bezüglich der Diskriminations- und Identifikationsfähigkeit von Gerüchen signifikant schlechter $a b(p=.01$ bzw. $p<.001)$. Obwohl die mittleren und medianen Riechschwellen eine deutliche Differenz (36 bzw. 70 \%) aufwiesen, bestand beim Schwellentest keine signifikanter Unterschied $(p=.08)$.

Tabelle 3-6: Ergebnisse des Riechtests in der Patienten- (PG) und Kontrollgruppe (KG)

\begin{tabular}{|c|c|c|c|c|c|c|c|c|}
\hline Variable & Gruppe & $\mathbf{N}$ & M & SD & Median & Min. & Max. & p-Wert \\
\hline \multirow{2}{*}{$\begin{array}{l}\text { Geruchs- } \\
\text { schwelle }\end{array}$} & PG & 29 & 5,6 & 6,3 & 2,5 & 0,0 & 16,0 & \multirow{2}{*}{.08} \\
\hline & KG & 9 & 8,8 & 5,0 & 8,3 & 2,8 & 16,0 & \\
\hline \multirow{2}{*}{$\begin{array}{l}\text { Diskriminations- } \\
\text { wert }\end{array}$} & $P G$ & 29 & 9,3 & 4,4 & 10,0 & 0,0 & 16,0 & \multirow{2}{*}{.01} \\
\hline & $K G$ & 10 & 13,3 & 3,2 & 14,0 & 7,0 & 16,0 & \\
\hline \multirow{2}{*}{$\begin{array}{l}\text { Identifikations- } \\
\text { wert }\end{array}$} & $P G$ & 29 & 8,8 & 3,4 & 10,0 & 3,0 & 16,0 & \multirow{2}{*}{$<.001$} \\
\hline & $K G$ & 10 & 12,9 & 1,8 & 13,5 & 9,0 & 15,0 & \\
\hline \multirow[t]{2}{*}{ TDI-Score } & $P G$ & 29 & 23,6 & 10,7 & 25,0 & 5,0 & 45,0 & \multirow{2}{*}{$<.001$} \\
\hline & $K G$ & 9 & 35,1 & 5,1 & 33,0 & 29,8 & 47,0 & \\
\hline
\end{tabular}

Deskriptive Kennwerte und Ergebnisse des Signifikanztests. Signifikante Ergebnisse sind grau hervorgehoben. Der TDI-Score ist abgeleitet aus den Ergebnissen der Subtests für Geruchsschwelle (engl. odor threshold), Diskrimination und Identifikation.

(M: Mittelwert; Max.: Maximum; Min.: Minimum; N: Stichprobenumfang; p-Wert: Signifikanzwert; SD: Standardabweichung)

Die Berechnung der TDI-Scores ergab folgendes Bild (siehe Abbildung 3-9 und Abbildung 3-10): Die Mittelwerte lagen bei den Patienten bei 23,6 und bei den Kontrollpersonen bei 35,1 (weitere Lage- und Streuungsmaße der Verteilung siehe Tabelle 3-6). Der Unterschied zwischen den Gruppen war signifikant $(p<.001)$. Acht der Patienten lagen im anosmischen Bereich, während sieben Patienten ein normales Riechvermögen hatten. 14 Patienten litten unter einer Hyposmie, die überwiegend mild (7 Patienten) und seltener moderat (3) oder schwer (4) ausgeprägt war. Unter den Kontrollpersonen waren 89 \% normosmisch. Bei einer Kontrollperson zeigte sich im Test eine milde Hyposmie. Anosmische, wie auch moderate oder schwere hyposmische Ergebnisse gab es in dieser Gruppe nicht. 
Die Sensitivität des TDI-Scores zur Unterscheidung zwischen Patienten und Kontrollpersonen lag bei $76 \%$, die Spezifität bei $88 \%$.

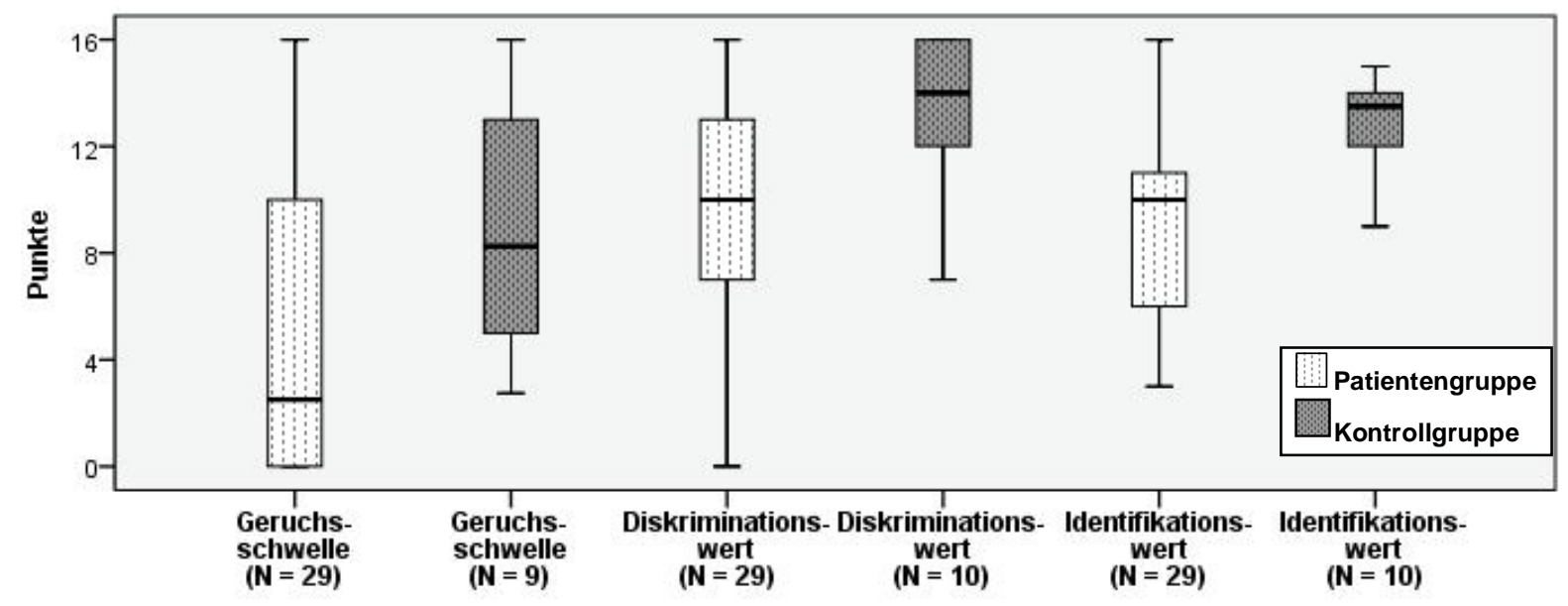

Abbildung 3-8: Boxplots zu den Ergebnissen der Patienten und Kontrollpersonen im Schwellen-, Diskriminations- und Identifikationstest

(N: Stichprobenumfang)

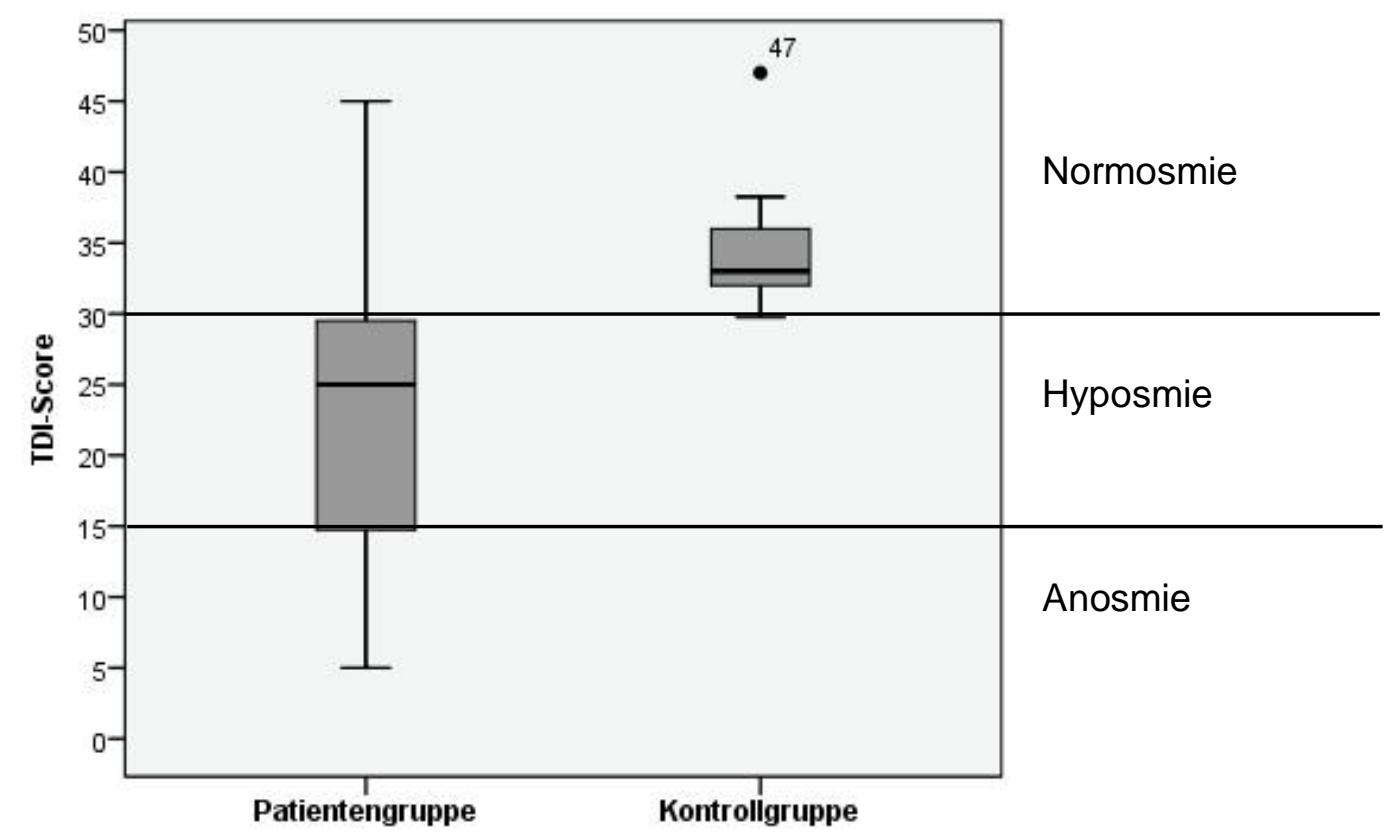

Abbildung 3-9: Boxplots zu den TDI-Scores in der Patienten- $(N=29)$ und Kontrollgruppe $(N=9)$.

Die Scores setzen sich zusammen aus den Ergebnissen der drei Subtests zu Geruchsschwelle (engl. odor threshold), Diskrimination und Identifikation.

(N: Stichprobenumfang) 


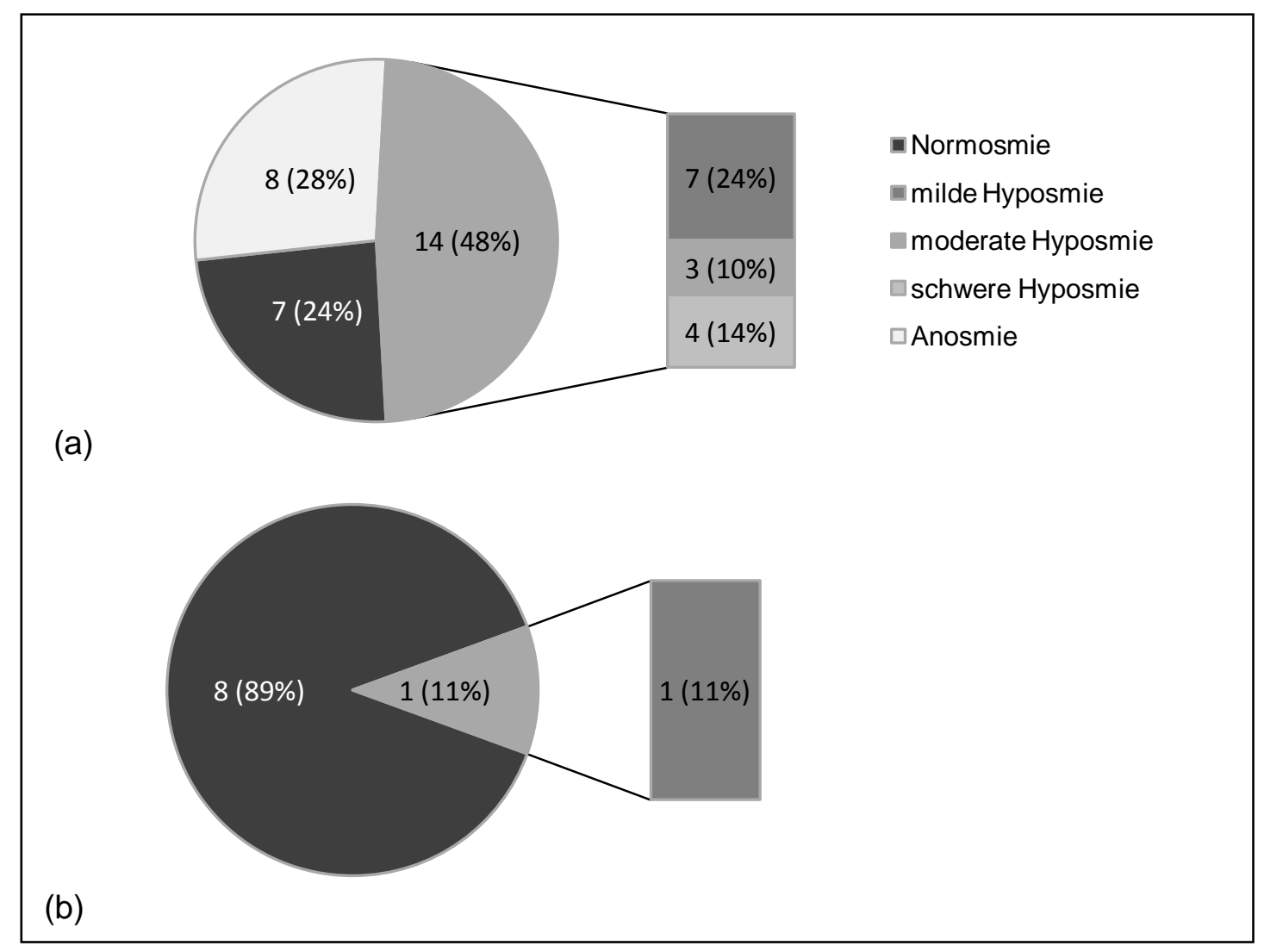

Abbildung 3-10: Riechfähigkeit der Patienten (a; $N=29)$ und Kontrollpersonen (b; $N=9)$ definiert durch die TDI-Scores.

Die Scores setzen sich zusammen aus den Ergebnissen der drei Subtests zu

Geruchsschwelle (engl. odor threshold), Diskrimination und Identifikation. Die Kreissegmente sind mit der absoluten Anzahl und dem prozentualen Anteil der fünf Ausprägungsgrade beschriftet.

(N: Stichprobenumfang)

\subsubsection{Schellongtest}

Sechs von neunundzwanzig Patienten und zwei von zehn Kontrollperson hatten im Schellongtest eine orthostatische Hypotension. Ein Patient verweigerte die Durchführung des Tests.

Zwei Patienten hatten in beiden Blutdruckparametern eine pathologische Differenz zum Ausgangswert. Bei vier Patienten und einer Kontrollperson fiel der systolische Wert um über $20 \mathrm{mmHg}$, bei einer Kontrollperson der diastolische um über $10 \mathrm{mmHg}$ (siehe Abbildung 3-11).

Der Gruppenvergleich ergab keinen signifikanten Unterschied $(p=1.00)$. 


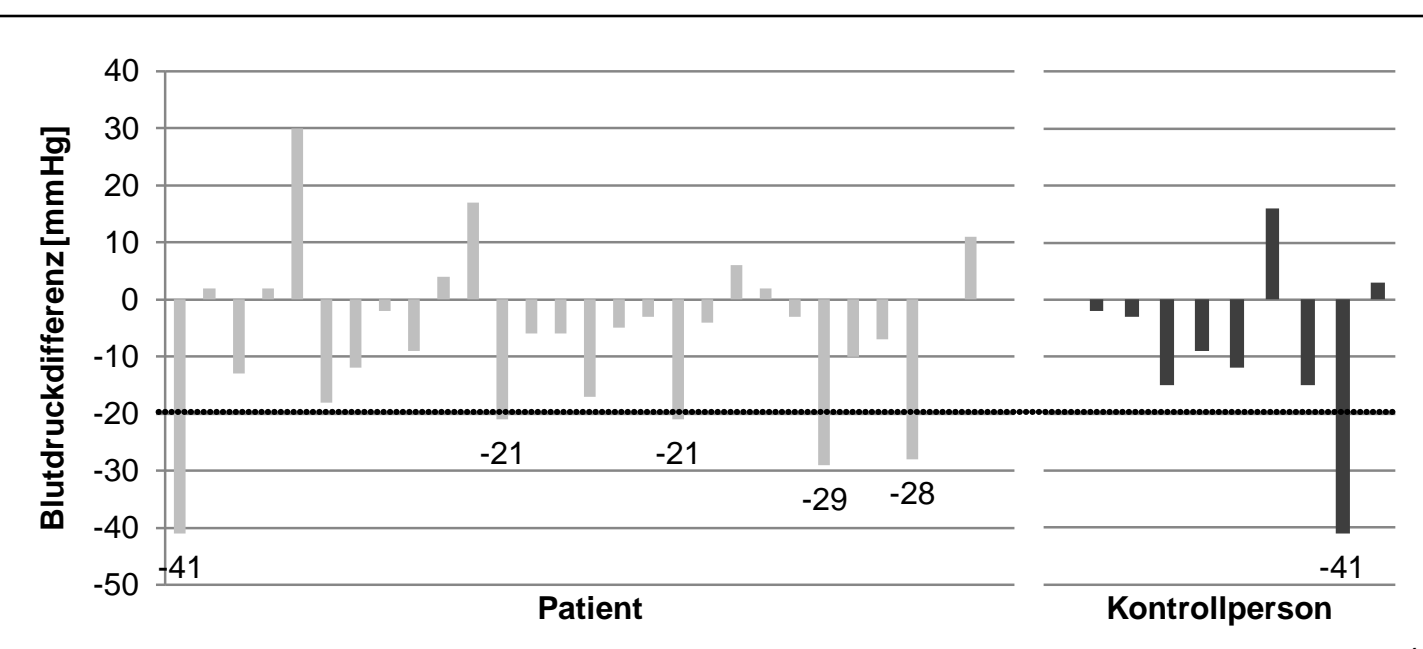

(a)

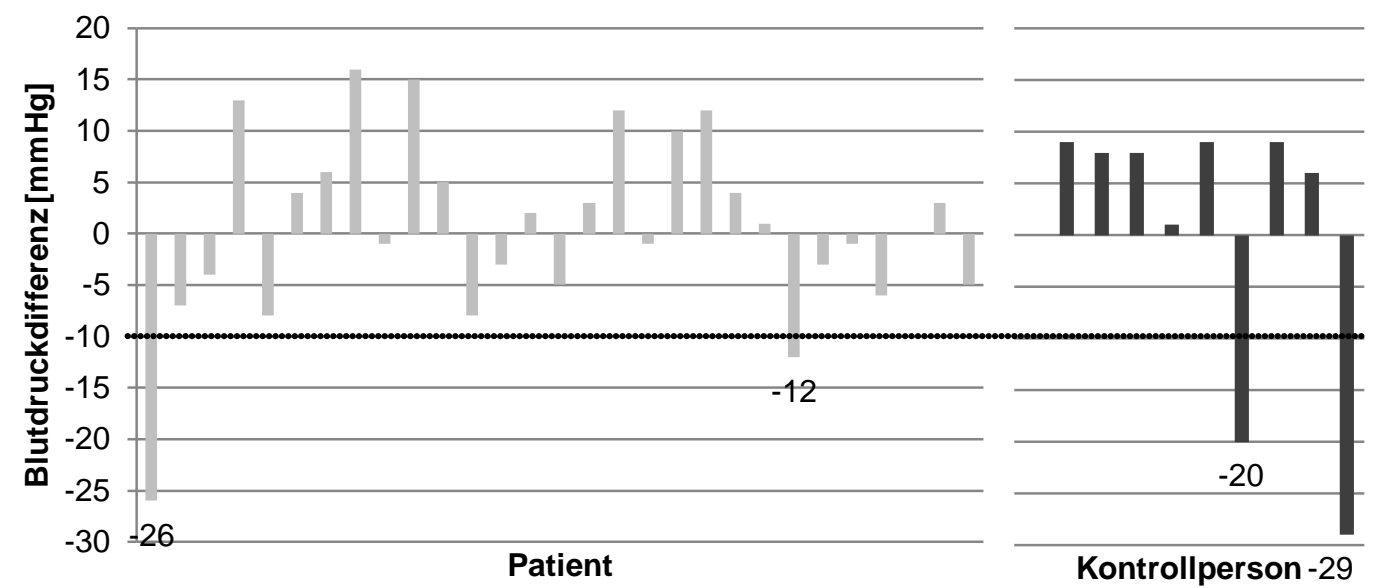

(b)

Abbildung 3-11: Differenzen der systolischen (a) und diastolischen (b) Blutdruckwerte der Patienten $(N=29)$ und Kontrollpersonen $(N=10)$ im Schellongtest.

Ab einem systolischen Abfall von $\geq 20 \mathrm{mmHg}$ oder einem diastolischen Abfall $\geq 10 \mathrm{mmHg}$ (gepunktete Linien) wird von einer orthostatischen Hypotension gesprochen.

( $N$ : Stichprobenumfang)

\subsubsection{Polysomnographie}

Die polysomnographischen Befunde der beiden untersuchten Gruppen sind in Abbildung 3-12 zu sehen. In Bezug auf das Vorliegen eines RWA bzw. einer RBD unterschieden sich die Patienten und gesunden Kontrollpersonen nicht signifikant voneinander (RBD: $p=.10$; RWA $p=.58)$. 
(a)

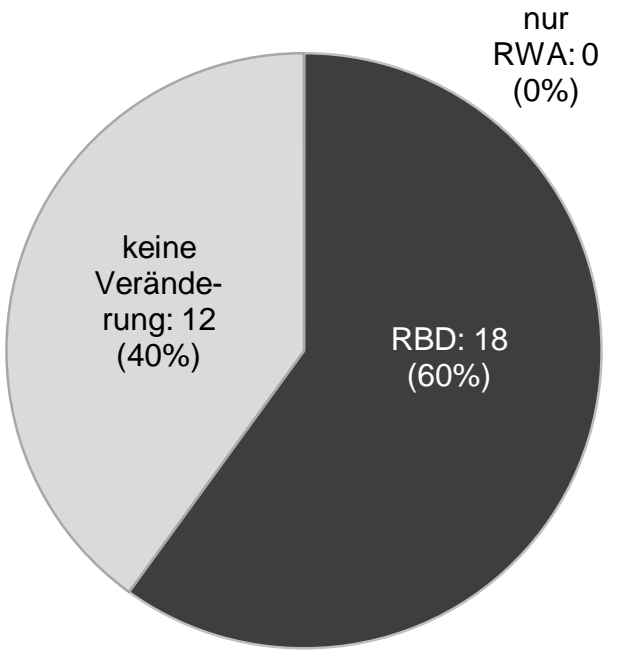

(b)

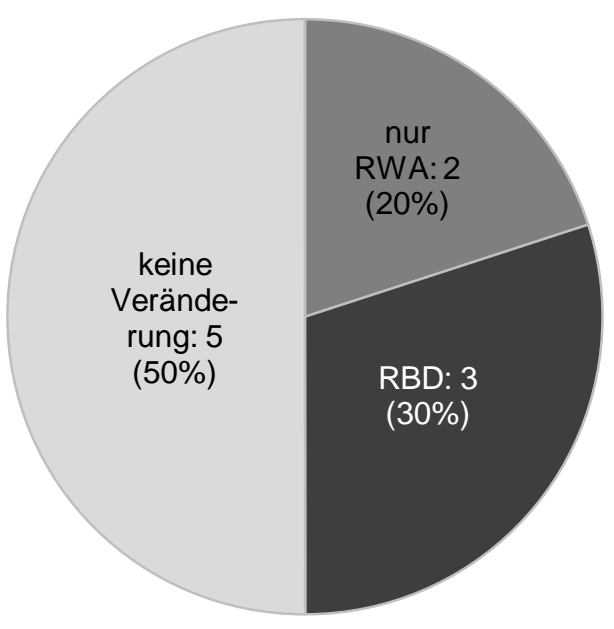

Abbildung 3-12: Absoluter und prozentualer Anteil von Personen ohne REM-SchlafVeränderungen, mit REM-Schlaf ohne Atonie (RWA) oder mit einer REM-SchlafVerhaltensstörung (RBD) in der Patienten- (a; $N=30)$ und Kontrollgruppe $(b ; N=10)$ (N: Stichprobenumfang; REM-Schlaf: Schlafphase mit schnellen Augenbewegungen)

Von den 18 Patienten mit RBD hatte einer Schweregrad 0.1, fünf Grad 1.0, drei Grad 1.1, fünf Grad 2.0, zwei Grad 2.1 und jeweils einer Grad 3.0 bzw. 3.1.

Bei den drei Kontrollpersonen wurde zweimal der Schweregrad 2.0 und einmal der Grad 3.0 ermittelt. Zwei Kontrollpersonen zeigten in der Video-unterstützten Polysomnographie nur einen RWA ohne motorische Aktivitäten oder Vokalisationen, was dem RBD-Grad 0.0 entspricht (siehe Abbildung 3-13).

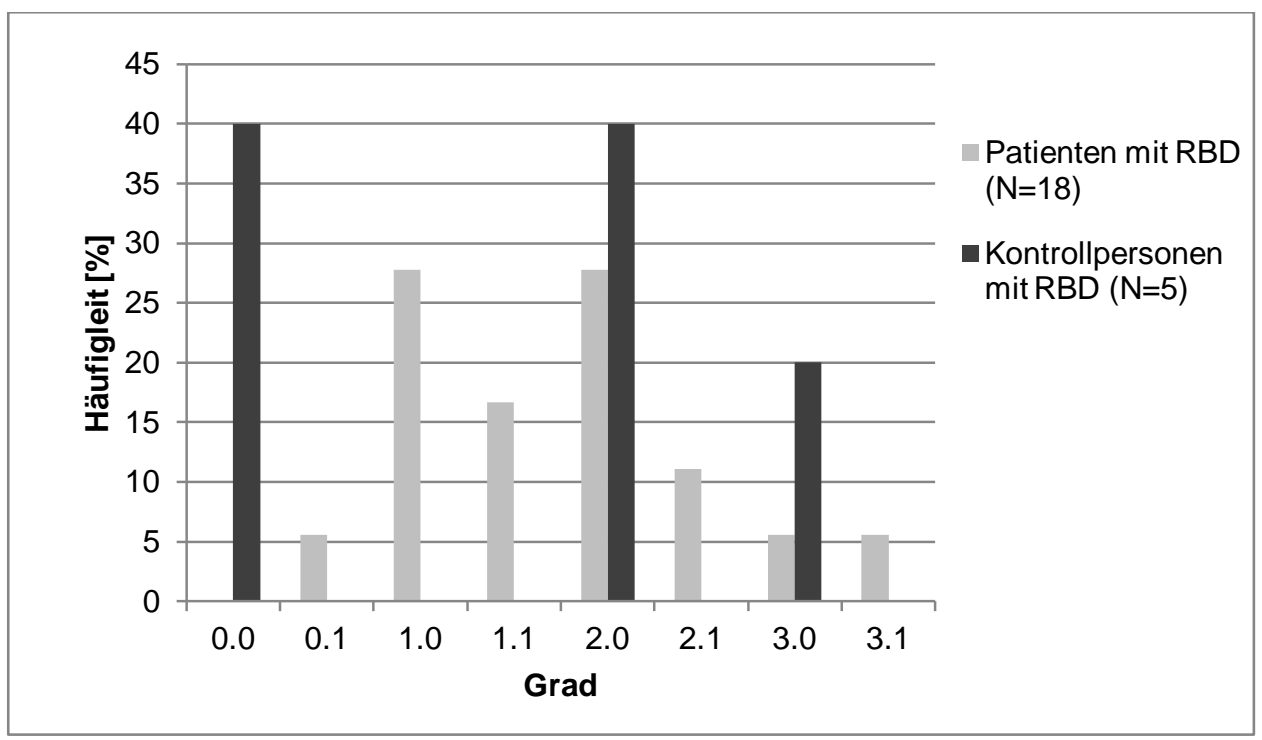

Abbildung 3-13: Verteilung der RBD-Grade in der Patienten- und Kontrollgruppe. RBD-Grad 0.0 entspricht dem ausschließlichen Vorhandensein eines RWA. (N: Stichprobenumfang; RBD: REM-Schlaf-Verhaltensstörung [REM-Schlaf: Schlafphase mit schnellen Augenbewegungen]; RWA: REM-Schlaf ohne Atonie) 


\subsubsection{Neuropsychologische Tests}

Der Mittelwert im MMST lag in der Patientengruppe bei 28,9 und in der Kontrollgruppe bei 29,1 Punkten. Beim Uhrentest hatten die Patienten im Mittel 2,0 Punkte, die Kontrollpersonen 1,8 Punkte. Bei einer Kontrollperson wurde kein Uhrentest durchgeführt. Acht Patienten (27\%) und zwei Kontrollpersonen (22\%) erzielten Ergebnisse über der Grenze von $\geq 3$ Punkten im Uhrentest, was für eine kognitive Beeinträchtigung spricht.

Alle weiteren Ergebnisse der deskriptiven Auswertung sind in Tabelle 3-7 zusammengefasst. Das Screening bezüglich kognitiver Einschränkungen ergab keine signifikanten Unterschiede zwischen Patienten und Kontrollpersonen, sowohl im MMST $(p=1.00)$ als auch im Uhrentest $(p=.06)$.

Tabelle 3-7: Ergebnisse des neuropsychologischen Screenings mit dem Mini-MentalStatus-Test (MMST) und Uhrentest in der Patienten- (PG) und Kontrollgruppe (KG)

\begin{tabular}{|l|l|r|r|r|r|r|r|r|}
\hline $\begin{array}{l}\text { Variable } \\
\text { [Einheit] }\end{array}$ & Gruppe & $\mathbf{N}$ & $\mathbf{M}$ & SD & Median & Min. & Max. & p-Wert \\
\hline \multirow{2}{*}{ MMST } & PG & 30 & 28,9 & 1,7 & 29 & 21 & 30 & \multirow{2}{*}{1.00} \\
\cline { 2 - 9 } & KG & 10 & 29,1 & 0,7 & 29 & 28 & 30 & \\
\hline \multirow{2}{*}{ Uhrentest } & PG & 30 & 2,0 & 1,2 & 2 & 1 & 5 & \multirow{2}{*}{06} \\
\cline { 2 - 9 } & KG & 9 & 1,8 & 1,1 & 1 & 1 & 4 & \\
\hline
\end{tabular}

Deskriptive Kennwerte und Ergebnisse des Signifikanztests.

(M: Mittelwert; Max.: Maximum; Min.: Minimum; N: Stichprobenumfang; p-Wert: Signifikanzwert; SD: Standardabweichung)

\subsection{Fragebögen}

Die Befragung der Studienteilnehmer erbrachte die in Tabelle 3-8 zusammengestellten Ergebnisse.

Im Folgenden wird auf die einzelnen Fragebögen eingegangen. 
Tabelle 3-8: Ergebnisse der Fragebogenerhebung in der Patienten- (PG) und Kontrollgruppe (KG)

\begin{tabular}{|c|c|c|c|c|c|c|c|c|}
\hline $\begin{array}{l}\text { Variable } \\
\text { [Einheit] }\end{array}$ & Gruppe & $\mathbf{N}$ & M & SD & Median & Min. & Max. & p-Wert \\
\hline \multirow{2}{*}{$\begin{array}{l}\text { NMSQuest } \\
{[\%]}\end{array}$} & $P G$ & 29 & 23,2 & 14,2 & 23,3 & 0,0 & 57,0 & \multirow{2}{*}{$<.001$} \\
\hline & KG & 10 & 8,3 & 7,6 & 6,7 & 3,0 & 27,0 & \\
\hline \multirow[t]{2}{*}{ NMSScale } & $P G$ & 26 & 32,8 & 34,8 & 17,5 & 0,0 & 131,0 & \multirow{2}{*}{.02} \\
\hline & KG & 10 & 9,3 & 13,0 & 5,5 & 0,0 & 45,0 & \\
\hline \multirow[t]{2}{*}{ PDQ-39SI } & $P G$ & 22 & 16,6 & 12,8 & 13,1 & 0,0 & 45,5 & \multirow{2}{*}{$<.001$} \\
\hline & KG & 9 & 1,7 & 2,6 & 0,0 & 0,0 & 6,4 & \\
\hline \multirow[t]{2}{*}{ RBDSQ } & $P G$ & 18 & 2,4 & 1,7 & 2,0 & 0,0 & 6,0 & \multirow{2}{*}{$<.001$} \\
\hline & $\mathrm{KG}$ & 7 & 0,6 & 0,8 & 0,0 & 0,0 & 2,0 & \\
\hline
\end{tabular}

Deskriptive Kennwerte und Ergebnisse des Signifikanztests. Signifikante Ergebnisse sind grau hervorgehoben.

(M: Mittelwert; Max.: Maximum; Min.: Minimum; N: Stichprobenumfang; NMSQuest: Non-Motor Symptoms Questionnaire; NMSScale: Non-Motor Symptoms Scale; PDQ-39SI: Parkinson's Disease Questionnaire Summary Index; p-Wert: Signifikanzwert; RBDSQ: RBD Screening Questionnaire [RBD: REM-Schlaf-Verhaltensstörung; REM-Schlaf: Schlafphase mit schnellen Augenbewegungen]; SD: Standardabweichung)

\subsubsection{NMSQuest}

Die Erhebung des NMSQuest ergab, dass bei den Patienten im Durchschnitt 23,2 \% (7/30) der NMS vorlagen. Die Kontrollpersonen bejahten durchschnittlich $8 \%(2,4 / 30)$ der NMS. Nur ein Patient gab an, keines der NMS aufzuweisen. Bei einem Patienten fehlten Angaben, sodass die Gruppenstärke der Patienten bei 29 lag. Die Signifikanz betrug $p<.001$.

Am häufigsten bei den Patienten waren die die Miktion betreffenden Symptome (Harndrang und Nykturie; jeweils $69 \%$ ) und etwa die Hälfte der Patienten litt an Vergesslichkeit (52 \%), Konzentrationsproblemen (48\%) und Ein- oder Durchschlafstörungen (48\%; siehe Tabelle 3-9). Auch bei den gesunden Kontrollpersonen waren diese Probleme am häufigsten, traten aber im Vergleich insgesamt seltener auf als bei den Patienten. Harndrang und Nykturie sowie Vergesslichkeit wurden fast doppelt so oft von den Patienten angegeben und Konzentrationsschwierigkeiten sogar fünf Mal so häufig. Nur bei Ein- oder Durchschlafstörungen gab es fast keinen Unterschied. Am deutlichsten unterschied sich die Prävalenz von Veränderungen des Geschmacks und Geruchs und von Interessenverlust. Diese Probleme wurden von 45 bzw. 34 \% der Patienten, aber von keiner gesunden Person bejaht. Keiner der Probanden hatte während des letzten Monats Stuhlinkontinenz, Wahnvorstellungen oder Halluzinationen. Alle anderen NMS wurden von mindestens einem Patienten bejaht. 
Tabelle 3-9: Prävalenz nicht-motorischer Symptome in der Patienten- und Kontrollgruppe (in Prozent, gemessen durch den Non-Motor Symptoms Questionnaire)

\begin{tabular}{|c|c|c|c|}
\hline Frage & nicht-motorisches Symptom & $\begin{array}{r}\text { Patienten } \\
(N=29)\end{array}$ & $\begin{array}{r}\text { Kontroll- } \\
\text { personen } \\
(\mathrm{N}=10)\end{array}$ \\
\hline 1 & Speichelsekretion & $17 \%$ & $0 \%$ \\
\hline 2 & Geschmack/ Geruch & $45 \%$ & $0 \%$ \\
\hline 3 & Schlucken & $17 \%$ & $10 \%$ \\
\hline 4 & Erbrechen & $3 \%$ & $0 \%$ \\
\hline 5 & Obstipation & $24 \%$ & $10 \%$ \\
\hline 6 & Stuhlinkontinenz & $0 \%$ & $0 \%$ \\
\hline 7 & inkomplette Stuhlentleerung & $24 \%$ & $10 \%$ \\
\hline 8 & Harndrang & $69 \%$ & $40 \%$ \\
\hline 9 & Nykturie & $69 \%$ & $40 \%$ \\
\hline 10 & Schmerzen & $10 \%$ & $10 \%$ \\
\hline 11 & Gewicht & $7 \%$ & $0 \%$ \\
\hline 12 & Vergesslichkeit & $52 \%$ & $30 \%$ \\
\hline 13 & Interessenverlust & $34 \%$ & $0 \%$ \\
\hline 14 & Halluzinationen & $0 \%$ & $0 \%$ \\
\hline 15 & Konzentration & $48 \%$ & $10 \%$ \\
\hline 16 & Depression & $34 \%$ & $0 \%$ \\
\hline 17 & Angst & $10 \%$ & $0 \%$ \\
\hline 18 & Interesse an Geschlechtsverkehr & $17 \%$ & $10 \%$ \\
\hline 19 & Schwierigkeiten beim Geschlechtsverkehr & $14 \%$ & $0 \%$ \\
\hline 20 & Schwindel & $21 \%$ & $10 \%$ \\
\hline 21 & Stürze & $14 \%$ & $0 \%$ \\
\hline 22 & Tagesmüdigkeit & $3 \%$ & $0 \%$ \\
\hline 23 & Insomnie & $48 \%$ & $40 \%$ \\
\hline 24 & intensive Träume & $38 \%$ & $10 \%$ \\
\hline 25 & Ausagieren von Träumen & $21 \%$ & $10 \%$ \\
\hline 26 & Restless-Legs-Syndrom & $24 \%$ & $10 \%$ \\
\hline 27 & Beinödeme & $28 \%$ & $0 \%$ \\
\hline 28 & Schwitzen & $28 \%$ & $0 \%$ \\
\hline 29 & Diplopie & $3 \%$ & $0 \%$ \\
\hline 30 & Wahnvorstellungen & $0 \%$ & $0 \%$ \\
\hline
\end{tabular}

Prävalenzen $\geq 30 \%$ sind grau hervorgehoben.

( $N$ : Stichprobenumfang)

\subsubsection{NMSScale}

Die mittlere Punktzahl in der NMSScale lag bei den Patienten bei 32,8 Punkten und bei den Kontrollpersonen bei 9,3 Punkten. Zwei Patienten verneinten alle NMS. Vier Fragebögen von Patienten konnten wegen fehlender Antworten nicht einbezogen werden. Der Gruppenvergleich ergab einen signifikanten Unterschied $(p=.02)$.

Beim Vergleich der Mittelwerte in den neun Bereichen der NMS lagen die Symptome, welche die Miktion betreffen (Bereich 7), bei den Patienten an der Spitze. An zweiter und dritter Stelle standen Aufmerksamkeit und Gedächtnis (Bereich 5) sowie Stimmung und Kognition (Bereich 3). Die meisten dieser Probleme waren nicht sehr ausgeprägt, dafür aber „häufig“ vorhanden. Bei Betrachtung der einzelnen Fragen hatten außerdem Ein- und Durchschlafstörungen (Frage 5) und Veränderungen beim Riechen oder Schmecken (Frage 28) einen hohen Stellenwert (siehe Tabelle 3-10). 
Tabelle 3-10: Ausprägungsgrad nicht-motorischer Symptome in der Patientengruppe (gemessen durch die Non-Motor Symptoms Scale; $N=26$ )

\begin{tabular}{|c|c|c|c|c|}
\hline Bereich & Frage & nicht-motorisches Symptom & $\begin{array}{r}\text { Mittel- } \\
\text { wert }\end{array}$ & $\begin{array}{l}\text { Bereichs- } \\
\text { Mittelwert }\end{array}$ \\
\hline \multirow[t]{2}{*}{ 1. Kardiovaskulär } & 1 & Benommenheit & 1,0 & \multirow{2}{*}{0,6} \\
\hline & 2 & Ohnmacht & 0,2 & \\
\hline \multirow[t]{4}{*}{ 2. Schlaf/ Müdigkeit } & 3 & Tagesmüdigkeit & 0,2 & \multirow{4}{*}{1,1} \\
\hline & 4 & Erschöpfung & 1,0 & \\
\hline & 5 & Insomnie & 2,7 & \\
\hline & 6 & Restless-Legs-Syndrom & 0,6 & \\
\hline \multirow[t]{6}{*}{ 3. Stimmung } & 7 & Interessenverlust & 1,3 & \multirow{6}{*}{1,3} \\
\hline & 8 & Motivationsmangel & 1,7 & \\
\hline & 9 & Nervosität & 0,9 & \\
\hline & 10 & Traurigkeit & 1,4 & \\
\hline & 11 & abgeflachter Affekt & 0,9 & \\
\hline & 12 & Mangel an Freude & 1,5 & \\
\hline \multirow{3}{*}{$\begin{array}{l}\text { 4. Wahrnehmungsproblemel } \\
\text { Halluzinationen }\end{array}$} & 13 & Halluzinationen & 0,0 & \multirow{3}{*}{0,0} \\
\hline & 14 & Wahrnehmungsstörungen & 0,0 & \\
\hline & 15 & Doppelbilder & 0,1 & \\
\hline \multirow{3}{*}{$\begin{array}{l}\text { 5. Aufmerksamkeit/ } \\
\text { Gedächtnis }\end{array}$} & 16 & Konzentration & 2,2 & \multirow{3}{*}{1,9} \\
\hline & 17 & Vergessen von Fakten/ Ereignissen & 2,2 & \\
\hline & 18 & Vergessen von Tätigkeiten & 1,4 & \\
\hline \multirow[t]{3}{*}{ 6. Gastrointestinaler Trakt } & 19 & Speichelfluss & 0,5 & \multirow{3}{*}{0,5} \\
\hline & 20 & Schluckstörungen & 0,6 & \\
\hline & 21 & Obstipation & 0,5 & \\
\hline \multirow[t]{3}{*}{ 7. Miktion } & 22 & Harndrang & 2,7 & \multirow{3}{*}{2,8} \\
\hline & 23 & Pollakisurie & 2,6 & \\
\hline & 24 & Nykturie & 3,2 & \\
\hline \multirow[t]{2}{*}{ 8. $\quad$ Sexualfunktion } & 25 & Interesse an Geschlechtsverkehr & 0,3 & \multirow{2}{*}{0,3} \\
\hline & 26 & Schwierigkeiten beim Geschlechtsverkehr & 0,2 & \\
\hline \multirow[t]{4}{*}{ 9. Verschiedenes } & 27 & Schmerzen & 0,6 & \multirow{4}{*}{0,8} \\
\hline & 28 & Geruch/ Geschmack & 1,8 & \\
\hline & 29 & Gewicht & 0,0 & \\
\hline & 30 & Schwitzen & 0,9 & \\
\hline
\end{tabular}

Es wurde für jedes Symptom das Produkt aus der individuellen Wertung von Häufigkeit und Schwere berechnet und aus den Ergebnissen aller Patienten der Mittelwert gebildet. Die Ergebnisse der Symptome eines Bereichs wurden anschließend zu einem BereichsMittelwert zusammengefasst. Die fünf höchsten Symptom-Mittelwerte und die drei höchsten Bereichs-Mittelwerte sind grau hervorgehoben.

( N: Stichprobenumfang)

\subsubsection{PDQ-39}

Die Auswertung des PDQ-39 umfasste 31 Fragebögen. Neun (acht von Patienten, einer von einer Kontrollperson) waren unvollständig ausgefüllt. Die Missings waren zufällig verteilt, d. h. es gab keine Fragen, die regelhaft unbeantwortet blieben. Der Mittelwert des PDQ-39SI lag in der Patientengruppe bei 16,6, in der Kontrollgruppe hingegen bei 0,6. In der Kontrollgruppe hatten fünf Personen (55,6 \%) einen Index von 0, in der Patientengruppe waren es zwei (9,1\%). Der Gruppenvergleich ergab einen signifikanten Unterschied $(p<.001)$. 
Über 50 \% der Patienten hatten zumindest „selten“ Einschränkungen aus den Bereichen Mobilität, Alltagsaktivitäten, emotionales Wohlbefinden, Kognition und körperliches Unbehagen. Am häufigsten angegeben wurde das Gefühl, ein schlechtes Gedächtnis zu haben, sich über die Zukunft Sorgen zu machen, sich deprimiert zu fühlen und Schwierigkeiten beim deutlichen Schreiben zu haben. Probleme mit dem Schreiben waren bei $41 \%$ der Patienten "häufig“ oder "immer" vorhanden genauso wie Zukunftssorgen bei $23 \%$. Schmerzen hatten $64 \%$ der Patienten, davon fast die Hälfte (27\%) „häufig“ oder „immer“. Es gab keine Frage, die nicht wenigstens ein Patient mit „selten“ beantwortet hätte. Am seltensten wurde angegeben, man habe eine Begleitperson benötigt (1 Patient) oder sich in der Öffentlichkeit wegen der Erkrankung geschämt (2 Patienten) (siehe Tabelle 3-11). Die Kontrollpersonen gaben in allen abgefragten Aspekten der Lebensqualität weniger häufig Einschränkungen an als die Patienten. Die meisten Beeinträchtigungen gab es in den Bereichen Kognition (Konzentration und Gedächtnis) und körperliches Behagen (Krämpfe und Schmerzen). Insgesamt waren aber auch diese Einschränkungen viel seltener und weniger stark ausgeprägt als bei den Patienten. Der Unterschied in der Häufigkeit von Problemen war am größten bei der Frage nach Schwierigkeiten bei der Ausübung von Freizeitaktivitäten: Diese kamen bei fast $60 \%$ der Patienten zumindest „selten“ vor, wurden aber von keiner Kontrollperson bejaht (siehe Tabelle 3-11).

\subsubsection{RBDSQ}

Beim RBDSQ erreichten die Patienten einen mittleren Score von 2,4 Punkten. Von den gesunden Kontrollpersonen verneinten $57 \%$ das Vorliegen aller abgefragten Charakteristika der RBD. Die übrigen bejahten eine oder zwei der Fragen. Somit lag der Mittelwert in dieser Gruppe bei 0,6 und die Signifikanz für den Gruppenvergleich bei $p<.001$.

Von den 40 Personen, die den Fragebogen zu REM-Schlafverhaltensstörungen zur Selbstbeurteilung bekamen, füllten 12 Patienten und 3 Kontrollpersonen diesen nicht vollständig aus, sodass diese Fragebögen nicht in die Auswertung einbezogen wurden. Lücken traten gehäuft auf bei den Fragen 10 (Vorliegen einer Erkrankung des Nervensystems; 8 Missings) und 6.1 (lautes Sprechen, Schreien, Schimpfen, Lachen während des Träumens; 7 Missings).

Am häufigsten gaben die Patienten an, ihnen sei bekannt, dass sie ihre Arme oder Beine im Schlaf bewegten (7/18), sie könnten sich gut an den Inhalt ihrer Träume erinnern (5/18) und sie hätten teilweise sehr lebhafte Träume (4/18). Diese drei Fragen waren gleichzeitig die einzigen, die von einzelnen Kontrollpersonen bejaht wurden. 
Tabelle 3-11: Beeinträchtigung von Aspekten der Lebensqualität (gemessen durch den Parkinson's Disease Questionnaire (PDQ-39))

\begin{tabular}{|c|c|c|c|c|}
\hline \multirow[t]{2}{*}{ Bereich/ Frage } & \multicolumn{2}{|c|}{$\begin{array}{l}\text { Häufigkeit positiver } \\
\text { Antworten [\%] }\end{array}$} & \multicolumn{2}{|c|}{$\begin{array}{c}\text { Häufigkeit der } \\
\text { Antwortmöglichkeiten } \\
\text { „häufig“ oder, „immer“ [\%] }\end{array}$} \\
\hline & $\begin{array}{r}\text { Patienten- } \\
\text { gruppe } \\
(\mathrm{N}=22)\end{array}$ & $\begin{array}{r}\text { Kontroll- } \\
\text { gruppe } \\
(\mathrm{N}=9)\end{array}$ & $\begin{array}{r}\text { Patienten- } \\
\text { gruppe } \\
(\mathrm{N}=22)\end{array}$ & $\begin{array}{r}\text { Kontroll- } \\
\text { gruppe } \\
(\mathrm{N}=9)\end{array}$ \\
\hline \multicolumn{5}{|l|}{ Mobilität } \\
\hline 1. $\quad$ Freizeitaktivitäten & 59 & 0 & 18 & 0 \\
\hline 2. Haushaltstätigkeiten & 41 & 0 & 0 & 0 \\
\hline 3. Einkaufen & 41 & 0 & 5 & 0 \\
\hline 4. $1 \mathrm{~km}$ gehen & 36 & 0 & 9 & 0 \\
\hline 5. $100 \mathrm{~m}$ gehen & 23 & 0 & 0 & 0 \\
\hline 6. im Haus bewegen & 27 & 0 & 5 & 0 \\
\hline 7. in der Öffentlichkeit bewegen & 36 & 0 & 9 & 0 \\
\hline 8. Begleitperson notwendig & 5 & 0 & 0 & 0 \\
\hline 9. Angst vorm Fallen & 23 & 0 & 9 & 0 \\
\hline 10. ans Haus gebunden sein & 32 & 0 & 5 & 0 \\
\hline \multicolumn{5}{|l|}{ Alltagsaktivitäten } \\
\hline 11. Probleme beim Waschen & 14 & 0 & 0 & 0 \\
\hline 12. Probleme beim Anziehen & 14 & 0 & 5 & 0 \\
\hline 13. Probleme beim Knöpfen & 41 & 0 & 9 & 0 \\
\hline 14. undeutliches Schreiben & 68 & 11 & 41 & 0 \\
\hline 15. Essen klein schneiden & 50 & 0 & 0 & 0 \\
\hline 16. Getränk verschütten & 45 & 11 & 9 & 11 \\
\hline \multicolumn{5}{|l|}{ emotionales Wohlbefinden } \\
\hline 17. deprimiert fühlen & 68 & 11 & 5 & 0 \\
\hline 18. einsam fühlen & 45 & 11 & 5 & 0 \\
\hline 19. verärgert sein & 36 & 11 & 14 & 0 \\
\hline 20. den Tränen nahe sein & 55 & 11 & 5 & 0 \\
\hline 21. ängstlich fühlen & 50 & 0 & 5 & 0 \\
\hline 22. Zukunftssorgen & 73 & 11 & 23 & 0 \\
\hline \multicolumn{5}{|l|}{ Stigma } \\
\hline 23. Krankheit verheimlichen & 36 & 0 & 0 & 0 \\
\hline 24. Situationen vermeiden & 45 & 0 & 14 & 0 \\
\hline 25. schämen & 9 & 0 & 0 & 0 \\
\hline 26. Sorgen über Reaktionen & 32 & 0 & 0 & 0 \\
\hline \multicolumn{5}{|l|}{ soziale Unterstützung } \\
\hline 27. Probleme mit Menschen & 27 & 0 & 5 & 0 \\
\hline 28. Unterstützung (Ehe-)Partner & 27 & 0 & 0 & 0 \\
\hline 29. Unterstützung Freunde & 32 & 0 & 5 & 0 \\
\hline \multicolumn{5}{|l|}{ Kognition } \\
\hline 30. tagsüber einschlafen & 23 & 0 & 0 & 0 \\
\hline 31. Konzentrationsprobleme & 55 & 33 & 9 & 0 \\
\hline 32. schlechtes Gedächtnis & 77 & 22 & 18 & 0 \\
\hline 33. schlechte Träume & 32 & 0 & 9 & 0 \\
\hline \multicolumn{5}{|l|}{ Kommunikation } \\
\hline 34. Sprechschwierigkeiten & 41 & 0 & 9 & 0 \\
\hline 35. Kommunikationsprobleme & 36 & 0 & 9 & 0 \\
\hline 36. fehlende Beachtung & 23 & 0 & 0 & 0 \\
\hline \multicolumn{5}{|l|}{ körperliches Unbehagen } \\
\hline 37. Muskelkrämpfe & 50 & 22 & 14 & 0 \\
\hline 38. Gelenkschmerzen & 64 & 33 & 27 & 0 \\
\hline 39. Hitze-/Kältegefühl & 32 & 0 & 9 & 0 \\
\hline
\end{tabular}

Angegeben ist der prozentuale Anteil der Studienteilnehmer, die das Vorliegen der jeweiligen Beeinträchtigung unabhängig von deren Häufigkeit bejahten (bei $\geq 50 \%$ grau markiert) sowie der Anteil der Studienteilnehmer, bei denen die jeweilige Beeinträchtigung häufig oder immer vorlag (bei $\geq 20 \%$ grau markiert).

( $N$ : Stichprobenumfang) 
Von den 18 Patienten und 7 Kontrollpersonen mit gültigen Fragebögen hatten 61 bzw. 43 \% eine polysomnographisch gesicherte RBD. Nur zwei Studienteilnehmer (beides Patienten) hatten aber ein positives Ergebnis im Screening auf RBD, alle anderen ein negatives. Die Übereinstimmung zwischen den Ergebnissen des RBDSQ und den polysomnographischen Befunden zum Vorliegen einer RBD ist Tabelle 3-12 dargestellt.

Tabelle 3-12: Vierfeldertafel zur Übereinstimmung des polysomnographischen Befundes und des RBD Screening Questionnaires (RBDSQ) bezüglich des Vorliegens einer REM-Schlaf-Verhaltensstörung (RBD)

\begin{tabular}{|ll|l|l|}
\cline { 3 - 3 } \multicolumn{1}{c|}{} & \multicolumn{2}{c|}{ positiv } & \multicolumn{2}{c|}{ negativ } \\
\hline \multirow{3}{*}{\begin{tabular}{|l} 
Ergebnis der \\
Polysomnographie
\end{tabular}} & RBD & 2 Patienten & 9 Patienten \\
\cline { 2 - 4 } & & 3 Kontrollpersonen \\
\hline
\end{tabular}

(REM-Schlaf: Schlafphase mit schnellen Augenbewegungen)

\subsection{Liquordiagnostik}

Bei 27 (90\%) der Patienten und 6 (60\%) der Kontrollpersonen konnte Liquor cerebrospinalis gewonnen werden. Die übrigen lehnten die Punktion ab (ein Patient wegen einer lumbalen Voroperation). Die Ergebnisse der Liquor-Analyse sind in Tabelle 3-13 und Abbildung 3-14 dargestellt. Der Gruppenvergleich zeigte keine signifikanten Unterschiede in den Konzentrationen von A $\beta 42(p=.09)$, Tau $(p=.60)$ und P-Tau $(p=.77)$.

Tabelle 3-13: Liquorkonzentrationen von $\beta$-Amyloid $_{(1-42)}(A \beta 42)$, Gesamt-Tau-Protein (Tau) und hyperphosphoryliertem Tau-Protein (P-Tau) in der Patienten- (PG) und Kontrollgruppe (KG)

\begin{tabular}{|c|c|c|c|c|c|c|c|c|}
\hline $\begin{array}{l}\text { Variable } \\
\text { [Einheit] }\end{array}$ & Gruppe & $\mathbf{N}$ & M & SD & Median & Min. & Max. & p-Wert \\
\hline \multirow{2}{*}{$\begin{array}{l}\text { Aß42 } \\
{[\mathrm{pg} / \mathrm{ml}]}\end{array}$} & PG & 27 & 625 & 232 & 585 & 188 & 1148 & \multirow{2}{*}{.09} \\
\hline & $K G$ & 6 & 820 & 211 & 801 & 536 & 1120 & \\
\hline \multirow{2}{*}{$\begin{array}{l}\text { Tau } \\
{[\mathrm{pg} / \mathrm{ml}]}\end{array}$} & $P G$ & 27 & 164 & 111 & 138 & 75 & 560 & \multirow{2}{*}{.60} \\
\hline & $K G$ & 6 & 129 & 38 & 135 & 75 & 173 & \\
\hline \multirow{2}{*}{$\begin{array}{l}\text { P-Tau } \\
\text { [pg/ml] }\end{array}$} & $P G$ & 27 & 55 & 28 & 52 & 23 & 137 & \multirow{2}{*}{.77} \\
\hline & $\mathrm{KG}$ & 6 & 48 & 6 & 50 & 36 & 53 & \\
\hline
\end{tabular}

Deskriptive Kennwerte und Ergebnisse des Signifikanztests.

(M: Mittelwert; Max.: Maximum; Min.: Minimum; N: Stichprobenumfang; p-Wert: Signifikanzwert; SD:

Standardabweichung) 
- ERGEBNISSE -

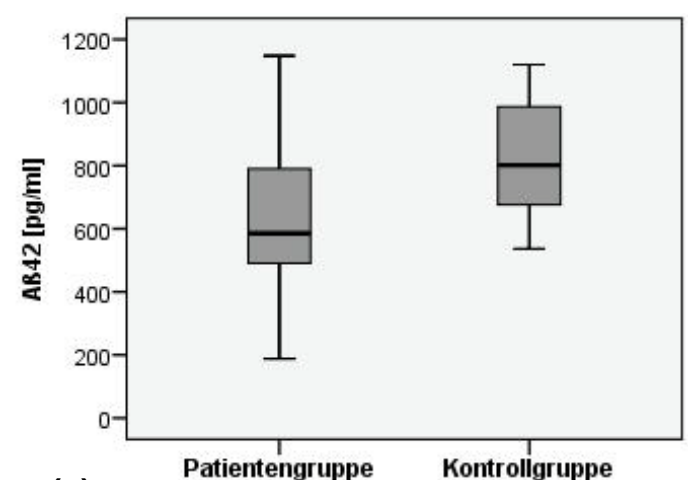

(a)

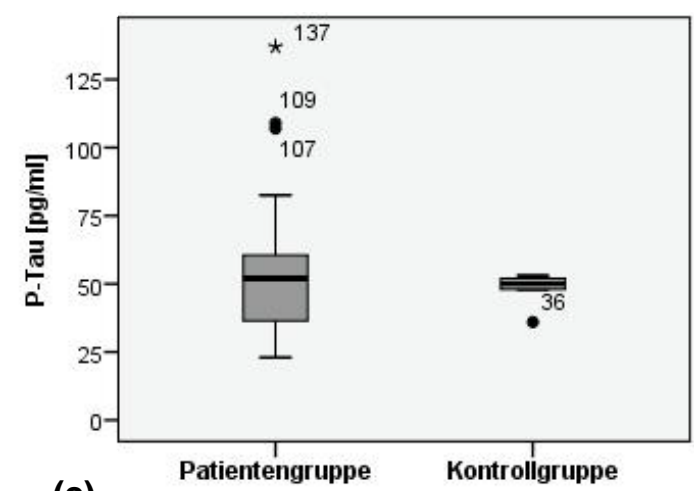

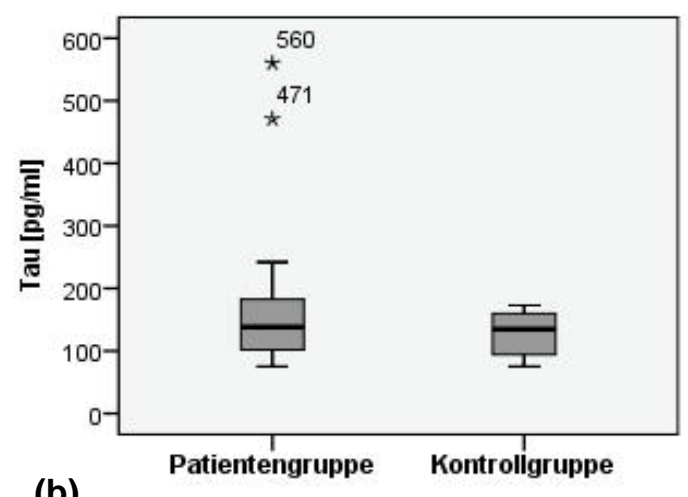

(b)

(c)

Abbildung 3-14: Boxplots zu den Konzentrationen von $\beta$-Amyloid ${ }_{(1-42)}(A \beta 42 ; a)$, Gesamt-Tau-Protein (Tau; b) und hyperphosphoryliertem Tau-Protein (P-Tau; c) im Liquor cerebrospinalis der Patienten $(N=27)$ und Kontrollpersonen $(N=6)$ ( $N$ : Stichprobenumfang)

\subsection{Feasibility}

In den ersten sieben Monaten seit Studienbeginn im September 2009 wurden insgesamt 36 Patienten und 11 Kontrollpersonen rekrutiert (siehe Abbildung 3-15).

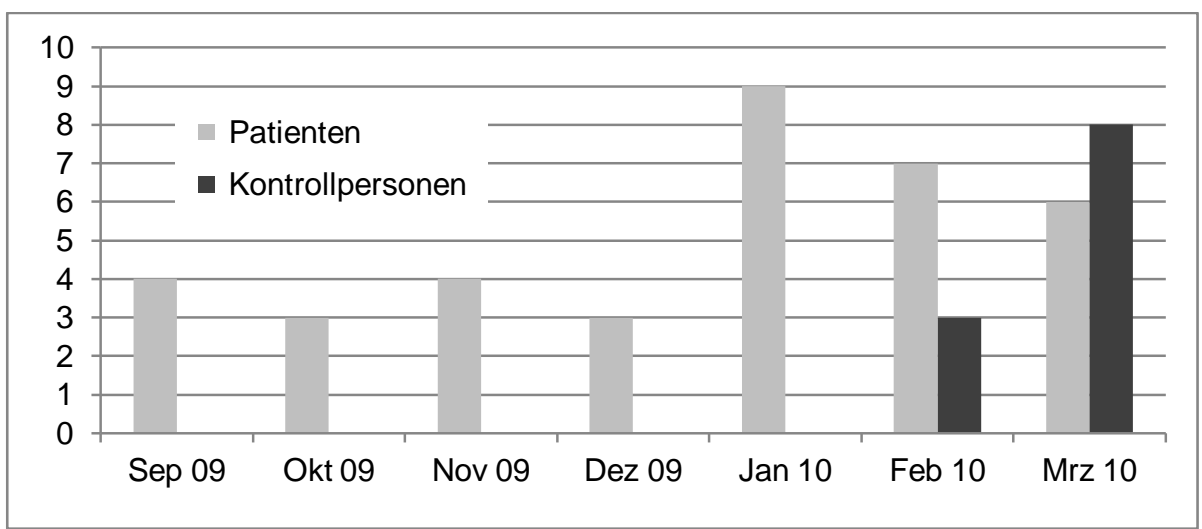

Abbildung 3-15: Monatliche Rekrutierungszahlen im Zeitraum von September 2009 bis März 2010 
Die Rekrutierung lag damit im Durchschnitt bei fünf Patienten pro Monat. Dieser Wert wurde im ersten Quartal von 2010 auf sieben gesteigert. Die maximale Rekrutierungszahl von Patienten lag bei neun (seit Studienbeginn) bzw. sieben (seit Beginn der Rekrutierung von Kontrollpersonen) pro Monat. Die Rekrutierung der Kontrollpersonen begann zeitversetzt im Februar 2010. Durchschnittlich wurden sechs, maximal acht Kontrollpersonen pro Monat rekrutiert.

Alle Untersuchungen konnten während eines dreitägigen stationären Aufenthalts durchgeführt werden. Kontrollpersonen wurden montags oder mittwochs aufgenommen und mittwochs bzw. freitags entlassen. Die Aufnahme von Patienten erfolgte meist montags. Der Klinikaufenthalt wurde gegebenenfalls unabhängig von der Studienteilnahme zur Einleitung einer medikamentösen Therapie verlängert.

Die Hochrechnung der Rekrutierungszahlen ergab, dass für das Erreichen der angestrebten Gesamtzahl von Probanden (150 Patienten und 100 Kontrollpersonen) bis Ende 2011 eine durchschnittliche Rekrutierung von sechs Patienten und fünf Kontrollpersonen pro Monat erforderlich ist (siehe Abbildung 3-16).

Auf einen Aufruf in einer Beilage "Gesundheit 2010" der regionalen Tageszeitung HNA meldeten sich im Januar 2010 bereits über 100 Freiwillige, die Interesse an der Studienteilnahme zeigten. Außerdem liefen und laufen noch andere Werbemaßnahmen, z. B. mittels Flyern und über die Homepage der Studie.

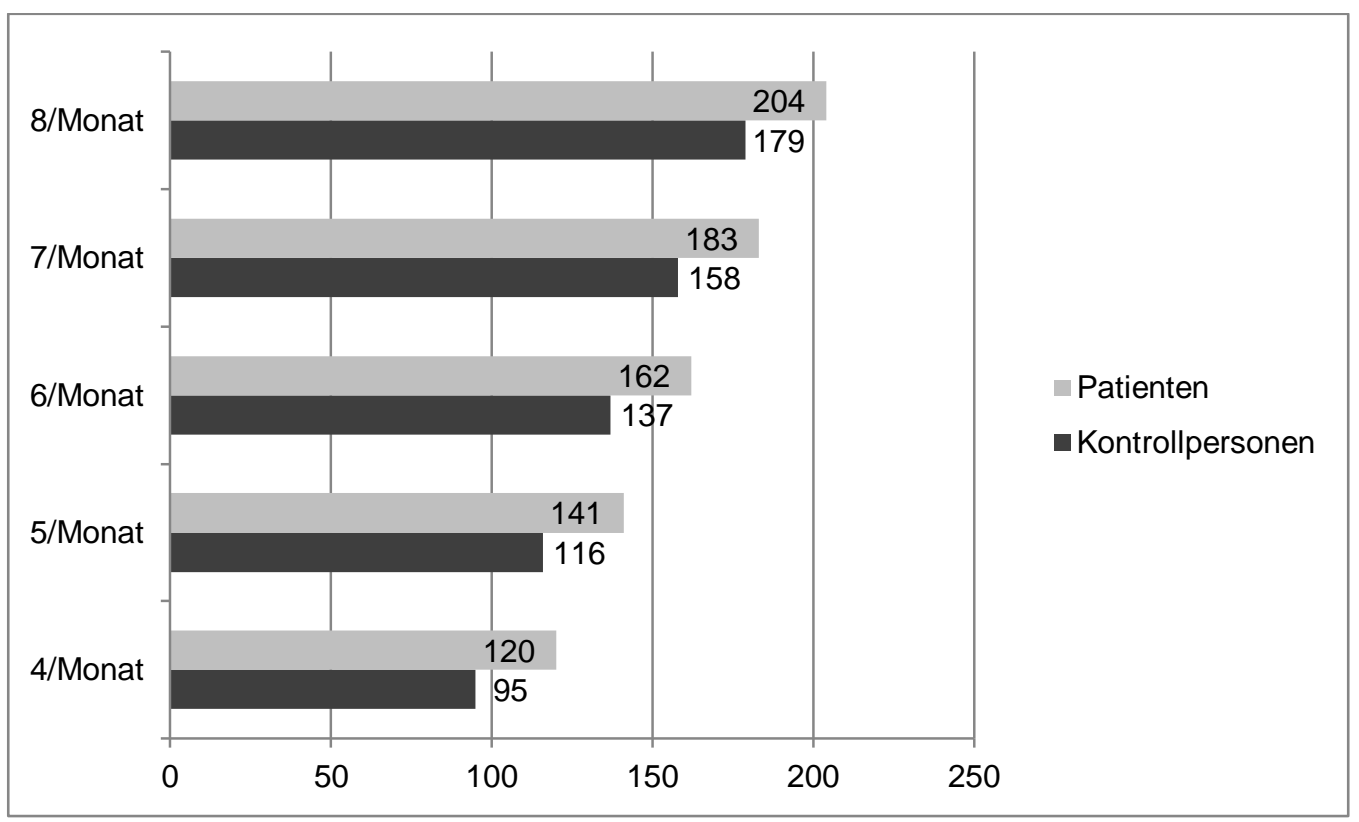

Abbildung 3-16: Hochrechnung der Probandenzahlen bis Ende Dezember 2011 ausgehend von einem Rekrutierungsstand von 36 Patienten und 11 Kontrollpersonen am 31. März 2010 bei einer durchschnittlichen monatlichen Rekrutierung von vier bis acht Patienten bzw. Kontrollpersonen.

Die angestrebten Zahlen von 150 Patienten und 100 Kontrollpersonen werden ab einer durchschnittlichen monatlichen Rekrutierung von 6 Patienten und 5 Kontrollpersonen erreicht. 
Neben den 30 De-novo-Parkinson-Patienten und den zehn Kontrollpersonen ohne vorbekannte neurologische Erkrankung wurden von September 2009 bis Ende März 2010 weitere sechs potenzielle Patienten und eine potenzielle Kontrollperson untersucht. Bei den Patienten war vor Einschluss von den einweisenden Ärzten der Verdacht auf IPS geäußert worden. Während des stationären Aufenthaltes zeigten sich jedoch Hinweise auf ein mögliches atypisches Parkinson-Syndrom oder eine andere Differenzialdiagnose des IPS (siehe Abbildung 3-17). Die Kontrollperson hatte eine vorbekannte Epilepsie und ein Restless-Legs-Syndrom (RLS). Wegen dieser Ausschlussgründe wurden die Daten der sieben Personen nicht in der Pilotstudie ausgewertet. Sie werden aber in der Hauptstudie als neurologische Kontrollpersonen („movement disorder controls“) dienen.

Zwischen April und Juni 2010 gab es auf Grund von verstärkten Bemühungen zum Ausschluss anderer Bewegungsstörungen beim Erstkontakt mit den Patienten keine weiteren Patienten, die nachträglich aus der Patientengruppe ausgeschlossen werden mussten. Der Prozentanteil in den ersten zehn Studienmonaten lag damit insgesamt bei $11 \%$.

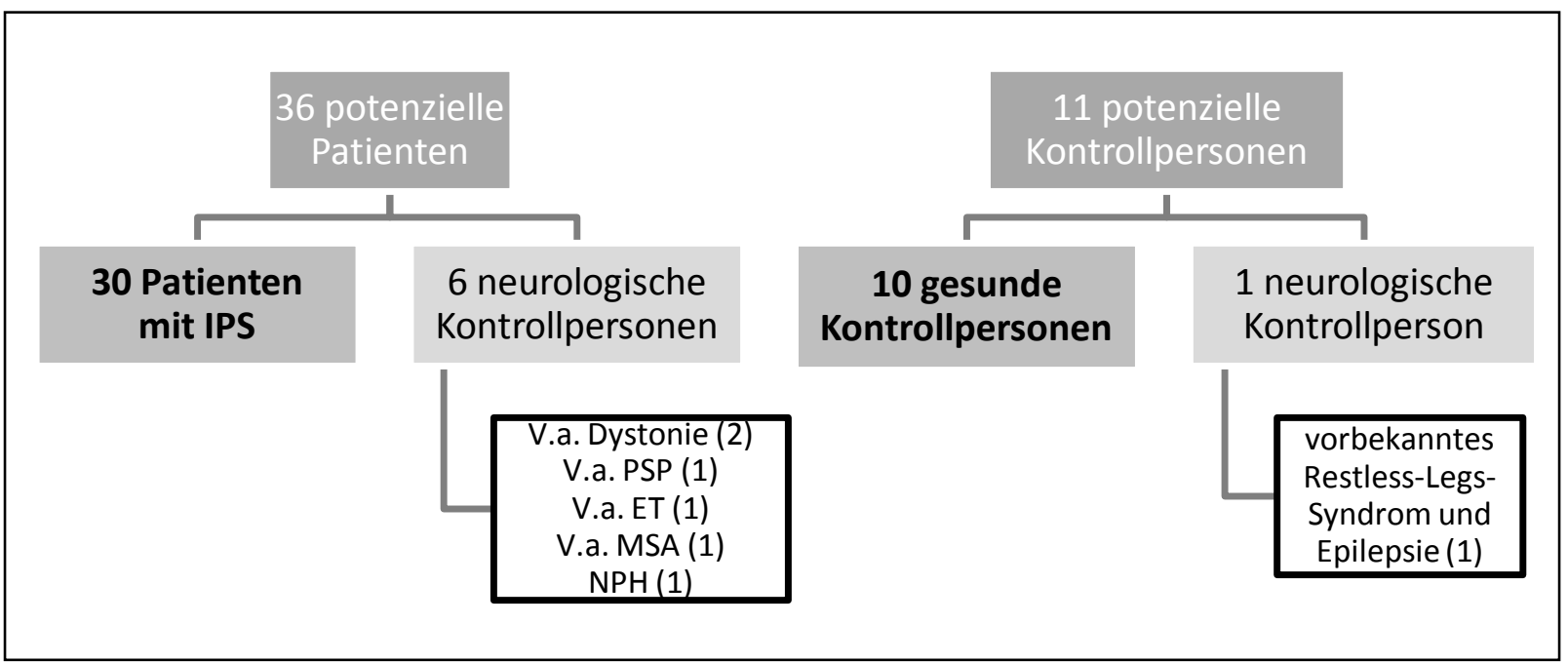

Abbildung 3-17: Flussdiagramm der von September 2009 bis März 2010 rekrutierten

\section{Studienteilnehmer.}

Bei 6 Patienten ergaben die Untersuchungen nach Einschluss Hinweise auf alternative Diagnosen. Eine Kontrollperson hatte vorbekannte neurologische Erkrankungen.

(ET: Essenzieller Tremor; IPS: Idiopathisches Parkinson-Syndrom; MSA: Multisystematrophie; NPH: Normaldruckhydrozephalus (engl. normal pressure hydrocephalus); PSP: progressive supranukleäre Blickparese) 
Eine Übersicht zur Vollständigkeit der erhobenen Daten liefert die folgende Tabelle.

Tabelle 3-14: Vollständigkeit der erhobenen Daten

\begin{tabular}{|c|c|c|}
\hline & Patientengruppe & Kontrollgruppe \\
\hline \multicolumn{3}{|l|}{ neurologische Untersuchung } \\
\hline UKPDSBB-Kriterien & $\checkmark$ & nicht vorgesehen \\
\hline Levodopa-Test & $\checkmark$ & nicht vorgesehen \\
\hline UPDRS & $\checkmark$ & $\checkmark$ \\
\hline H\&Y-Stadium & $\checkmark$ & $\checkmark$ \\
\hline \multicolumn{3}{|l|}{ Bildgebung } \\
\hline Hirnparenchymsonographie & $\begin{array}{l}\text { kein Schallfenster: } \\
2 \text { beidseits, } 2 \text { links }\end{array}$ & $\checkmark$ \\
\hline \multicolumn{3}{|l|}{ klinische Tests } \\
\hline Riechtest & $\begin{array}{l}\text { nicht durchgeführt: } \\
1 \text { komplett }\end{array}$ & $\begin{array}{l}\text { nicht durchgeführt: } \\
1 \text { Schwellentest }\end{array}$ \\
\hline Schellong-Test & nicht durchgeführt: 1 & $\checkmark$ \\
\hline Polysomnographie & $\checkmark$ & $\checkmark$ \\
\hline \multicolumn{3}{|l|}{ Neuropsychologische Tests } \\
\hline MMST & $\checkmark$ & $\checkmark$ \\
\hline Uhrentest & nicht durchgeführt: 1 & $\checkmark$ \\
\hline \multicolumn{3}{|l|}{ Fragebögen } \\
\hline NMSQuest & unvollständig: 1 & $\checkmark$ \\
\hline NMSScale & unvollständig: 4 & $\checkmark$ \\
\hline PDQ-39 & unvollständig: 8 & unvollständig: 1 \\
\hline RBDSQ & unvollständig: 12 & unvollständig: 3 \\
\hline Liquordiagnostik & nicht durchgeführt: 3 & nicht durchgeführt: 4 \\
\hline \multicolumn{3}{|c|}{$\begin{array}{l}\text { (V: Datensatz vollständig; H\&Y-Stadium: Stadium nach HOEHN und YAHR (1967); MMST: Mini- } \\
\text { Mental-Status-Test; NMSQuest: Non-Motor Symptoms Questionnaire; NMSScale: Non-Motor } \\
\text { Symptoms Scale; PDQ-39: Parkinson's Disease Questionnaire; RBDSQ: RBD Screening } \\
\text { Questionnaire [RBD: REM-Schlaf-Verhaltensstörung; REM-Schlaf: Schlafphase mit schnellen } \\
\text { Augenbewegungen]; UKPDSBB: United Kingdom Parkinson's Disease Society Brain Bank; UPDRS: } \\
\text { Unified Parkinson's Disease Rating Scale) }\end{array}$} \\
\hline
\end{tabular}




\section{DISKUSSION}

\subsection{Demographische Daten}

Die 30 Patienten und zehn Kontrollpersonen, deren Daten in dieser Pilotstudie ausgewertet wurden, unterschieden sich nicht signifikant in Bezug auf das Alter, das Geschlecht und den Bildungsstand, sodass die beiden Studiengruppen vergleichbar sind.

\subsection{Neurologische Untersuchung}

Alle Patienten hatten entsprechend der Diagnosekriterien der UKPDSBB ein ParkinsonSyndrom. Die Bradykinese wird dort obligat vorgeschrieben und alle Patienten dieser Pilotstudie erfüllten dieses Kriterium. Allerdings scheinen nicht zwangsläufig alle Patienten mit IPS eine Bradykinese zu entwickeln. LOUIS et al. (1997) untersuchten z. B. die klinischen Daten von Patienten mit pathologisch gesichertem IPS und fanden lediglich bei $95 \%$ eine Bradykinese. Daher fordern GELB et al. (1999) in den von innen vorgeschlagenen Diagnosekriterien, dass mindestens zwei der vier charakteristischen Symptome vorhanden sein müssten, darunter Ruhetremor oder/ und Bradykinese.

$85 \%$ der Patienten von LOUIS et al. (1997) bekamen im Laufe ihres Lebens einen Ruhetremor und 95,2 \% einen Rigor. In einer anderen klinisch-pathologischen Studie hatten $100 \%$ der Patienten einen Ruhetremor (RAJPUT et al. 1991 a). Die DeNoPa-Patienten zeigten erst zu jeweils $70 \%$ einen Rigor bzw. Ruhetremor. Niedrigere Prävalenzen zu Beginn der Krankheitsgeschichte wurden auch in anderen Untersuchungen beobachtet (HOEHN und YAHR 1967, PAPAPETROPOULOS et al. 2001).

Die posturale Instabilität war das seltenste der vier Hauptkriterien bei den DeNoPaPatienten. Diese wird eher als Komplikation des fortgeschrittenen IPS angesehen (SCHRAG et al. 2002 a, KLAWANS 1986). REICHMAN (2010) sagt sogar, dieses Symptom sei „....aufgrund der angestrebten Frühdiagnostik allerdings nicht mehr zeitgemäß“ (S. S5). CHASTAN et al. (2008) erfassten im Widerspruch dazu bei Untersuchungen mit einer Messplattform Zeichen einer gestörten posturalen Kontrolle bei Patienten mit frühem IPS im Stadium 1 oder 1,5, obwohl die Hälfte dieser Patienten einen unauffälligen Befund im Zugtest zeigten. Einschränkungen der Balance treten allerdings definitionsgemäß erst ab H\&Y-Stadium 3 auf. Patienten mit einseitiger Erkrankung dürften demnach keine Haltungsinstabilität haben (HOEHN und YAHR 1967).

In der vorliegenden Untersuchung waren die Symptome bei $80 \%$ der Patienten zu Beginn auf eine Körperhälfte beschränkt. Damit war ein einseitiger Beginn fast ebenso häufig wie in einer Studie von BARRETT et al. (2011) mit 1173 Parkinson-Patienten, von denen 86,5\% 
zunächst eine unilaterale Symptomatik zeigten. Schon HOEHN und YAHR (1967) beobachteten, dass die Krankheit bei einem Teil der Patienten bilateral oder generalisiert beginnt und damit das erste Stadium ihrer Schweregradeinteilung übersprungen wird. Dies schließt aber eine Asymmetrie im Verlauf nicht aus. Diese Seitendifferenz der Symptome persistiert bei den meisten Patienten (TOTH et al. 2004, LEE et al. 1995), was auch bei $80 \%$ der DeNoPa-Patienten im bisherigen Krankheitsverlauf der Fall war.

Obwohl es sich beim IPS um eine progrediente Erkrankung handelt, war bei $20 \%$ der Patienten (noch) kein Voranschreiten beobachtet worden. Dies könnte auf die zum Teil sehr kurze Zeit seit Beginn der Erkrankung zurückzuführen sein.

Der L-Dopa-Test war bei $77 \%$ der De-novo-Patienten positiv. Bei vier Patienten verbesserten sich die motorischen Symptome um weniger als $30 \%$ und bei drei Patienten zeigte die Einnahme von L-Dopa keinen Effekt. In einer Studie von HUGHES et al. (1992) zeigten trotz pathologisch gesicherter Diagnose sogar nur $26 \%$ von 76 Patienten ein exzellentes initiales Ansprechen auf L-Dopa. 4 \% sprachen nicht oder kaum an und $12 \%$ nur moderat. Fehlendes oder begrenztes Ansprechen bei Erkrankungsbeginn spricht also nicht grundsätzlich gegen die Diagnose eines IPS. Auch ein positives Ansprechen auf eine langfristige Behandlung mit L-Dopa wird dadurch nicht ausgeschlossen (HUGHES et al. 1990). Der L-Dopa-Test ist im frühen Stadium zudem schwierig zu interpretieren, da die Patienten noch niedrige Scores im Abschnitt III der UPDRS haben. Wenn die Symptomatik gering ausgeprägt ist und vor der Medikamenteneinnahme mit einem Punkt bewertet wurde, ist es auch bei einer sichtbaren Verbesserung eines Symptoms häufig nicht vertretbar, null Punkte zu vergeben, da dies „normal“ bedeuten würde.

Die mediane Erkrankungsdauer der Patienten vor Einschluss in die Studie lag bei 21,5 Monaten. Allerdings gab es auch anamnestische Angaben von bis zu 10 Jahren, was den Mittelwert auf 29,4 Monate anhob. Auf Grund des schleichenden Beginns der Erkrankung und der retrospektiven Erhebung, basierend alleine auf der Wahrnehmung der Patienten, handelt es sich dabei nur um Näherungen, nicht um exakte Angaben. Trotzdem beschrieben ELBAZ et al. (2003) eine ähnlich große Zeitspanne von 0 bis 9,7 Jahren für die Zeit zwischen Beginn der Erkrankung und Diagnosestellung bei Parkinson-Patienten. Die ersten Symptome des IPS bleiben oft für lange Zeit unbeachtet oder werden missinterpretiert, sodass die Patienten unter Umständen schon einige Jahre erkrankt sein können, bevor der Verdacht auf IPS aufkommt. Im Rückblick erinnern sich die Patienten oder deren Angehörige dann an potenzielle Frühsymptome, die schon lange Zeit bestehen (MARSDEN 1994). HARIZ und FORSGREN (2011) zeigten, dass sich Patienten mit Tremor-dominanter Erkrankung länger ihrer Symptome bewusst waren, bevor sie eine medizinische Beratung suchten, als Patienten mit anderen Erkrankungstypen. 
Obwohl die bestätigenden Diagnosekriterien teilweise auf Grund der begrenzten Krankheitsdauer von keinem Patienten erfüllt werden konnten, trafen bei $83 \%$ der Patienten schon drei oder mehr dieser Kriterien zu. Bei diesen Patienten stand damit die klinische Diagnose des IPS nach den Kriterien der UKPDSBB sicher fest.

Die Auswertung des UPDRS ergab, dass die Patienten trotz des frühen Krankheitsstadiums durch ihre Erkrankung signifikant stärker beeinträchtigt und behindert waren als gleichaltrige Kontrollpersonen. Dies galt sowohl für den Bereich der kognitiv-psychiatrischen Symptome (Abschnitt I) als auch für den Komplex der Aktivitäten des täglichen Lebens (Abschnitt II). Auch die motorische Untersuchung (Abschnitt III) erbrachte bei den Patienten ein signifikant schlechteres Ergebnis. Kognitive Einschränkungen, aber auch depressive und apathische Symptome wurden bereits mehrfach bei neu diagnostizierten, teilweise auch unbehandelten Parkinson-Patienten festgestellt, wobei die Häufigkeit größer war als in Kontrollgruppen (MUSLIMOVIC et al. 2005, AARSLAND et al. 2009 a und b, RAVINA et al. 2009). Insgesamt war die Ausprägung dieser Merkmale bei den DeNoPa-Patienten aber mild, da von den möglichen 16 Punkten nur maximal 6 und im Mittel 2 Punkte erreicht wurden. Psychotische Störungen wie Halluzinationen oder Wahnvorstellungen sind bei Patienten im frühen Erkrankungsstadium selten (AARSLAND et al. 2009 a) und waren entsprechend bei keinem der DeNoPa-Patienten vorhanden. Zunehmende Depression und sich verschlechternde Kognition sind assoziiert mit Behinderung bei alltäglichen Aktivitäten (WEINTRAUB et al. 2004) und können daher zu dem signifikant schlechteren Ergebnis der Patienten im Abschnitt II der UPDRS beigetragen haben.

Was die Fähigkeit angeht, den Anforderungen des Alltags gerecht zu werden, so berichteten auch HARIZ und FORSGREN (2011) von Limitationen bei unbehandelten ParkinsonPatienten zum Zeitpunkt der Diagnosestellung. Wie bei den DeNoPa-Patienten fand sich auch dort eine relativ große Streuung der Ergebnisse mit einem Range von 2-25 Punkten. Begründet scheint diese durch das Vorliegen verschiedener durch motorische Symptome definierter Subtypen der Parkinson-Erkrankung, die sich im Einfluss auf das alltägliche Leben unterscheiden. Die größten Einschränkungen erfahren Patienten mit überwiegend axialen Symptomen (ebd.).

Die frühe Präsentation des IPS beinhaltete bei den DeNoPa-Patienten besonders häufig feinmotorische Probleme. Diese könnten zumindest teilweise durch den bei über $80 \%$ der Patienten vorhandenen und häufig als störend eingestuften Tremor verursacht worden sein. Verschiedene Autoren beschrieben Schwierigkeiten beim Schreiben bzw. Veränderungen des Schriftbildes als frühes Charakteristikum des IPS (PONSEN et al. 2008, MARSDEN 1994). Diese Ansicht wird durch das Ergebnis der DeNoPa-Kassel-Pilotstudie bestätigt, da $83 \%$ der Patienten ebensolche Probleme bejahten. Bei HARIZ und FORSGREN (2011) gehörte das Schreiben zu den wenigen Aspekten, die nicht nur Patienten mit primär axialer 
Symptomatik, sondern auch Patienten mit Tremor-dominantem IPS signifikant von gesunden Kontrollpersonen unterschieden.

Da die Diagnostik des IPS auf dem Vorliegen motorischer Symptome basiert, war zu erwarten, dass die Patienten signifikant höhere Summenscores erreichten als die Kontrollpersonen. Der Median in der DeNoPa-Gruppe lag mit 19 von maximal 108 Punkten insgesamt im unteren Bereich. Die motorischen Symptome waren also noch mild ausgeprägt. Wie in Abschnitt II der UPDRS zeigten sich auch Abschnitt zur motorischen Untersuchung große interindividuelle Unterschiede bei den Patienten. Diese waren insgesamt aber nicht ganz so ausgeprägt wie bei HARIZ und FORSGREN (2011), die bis zu 62 Punkte ermittelten. Allerdings hatten dort auch $7 \%$ der unbehandelten, neu diagnostizierten Patienten bereits ein H\&Y-Stadium 4 oder 5, was per Definition eine starke Behinderung und große Abhängigkeit von betreuenden Personen bedeutet. Zum anderen spielt sicher auch der Anteil von Personen mit verschiedenen Erkrankungstypen eine Rolle. Die schlechtesten Scores hatten bei HARIZ und FORSGREN (2011) die Patienten mit primär axialer Symptomatik, die auch bei den Aktivitäten des täglichen Lebens unter den größten Einschränkungen litten.

Da alle Patienten unbehandelt waren, waren bei keinem Studienteilnehmer motorische oder nicht-motorische Therapie-Komplikationen zu erwarten, die Gegenstand von Abschnitt IV der UPDRS sind.

Die H\&Y-Stadien in der Patientengruppe zeigten, dass die Erkrankung bei allen Patienten leicht bis mäßig ausgeprägt und bei über der Hälfte der Patienten auf eine Körperhälfte begrenzt war.

\subsection{Hirnparenchymsonographie}

Korrespondierend zu den Ergebnissen anderer Studien (MEHNERT et al. 2010, WALTER et al. 2002) war der Unterschied zwischen Patienten- und Kontrollgruppe bezüglich der SNFlächen beidseits signifikant. Es konnte gezeigt werden, dass die Hyperechogenität der SN auch bei neu diagnostizierten Patienten ohne Behandlung mit einer Häufigkeit von 89 \% ein charakteristisches Merkmal darstellt. Dies unterstreicht, dass es sich bei der HPS um ein nützliches Instrument auch oder besonders in der Früherkennung des IPS handelt. Die HPS erscheint geeignet um IPS-Patienten von Gesunden abzugrenzen und so eine ParkinsonDiagnose zu bekräftigen. Zu diesem Ergebnis kamen auch GAENSLEN et al. (2008), die bei Patienten mit den ersten milden Symptomen eines IPS eine HPS durchführten und ein Jahr später nach Sicherung der Diagnose eine Sensitivität der HPS von 90,7\% und eine Spezifität von 82,4\% ermittelten. 
Allgemein sind die Vorteile der Sonographie darin zu sehen, dass sie kostengünstig, weit verbreitet und damit leicht verfügbar, beliebig wiederholbar und nicht schädigend für den menschlichen Organismus ist. Die Methode zeigt zudem eine geringe Untersucherabhängige Variabilität (BERG et al. 1999 a, MEHNERT et al. 2010).

Die gemessenen medianen Flächen unterschieden sich nur geringgradig von dem durch WALTER et al. (2002) für Parkinson-Patienten ermittelten Median von $0,25 \mathrm{~cm}^{2}$ für beide Seiten gemeinsam, obwohl in dieser Untersuchung die Erkrankungsdauer deutlich länger und der Ausprägungsgrad schwerer war als bei den DeNoPa-Patienten. Dies kann als Bestätigung für die Befunde von BERG et al. (2001 a und 2005) oder MEHNERT et al. (2010) gewertet werden. Diese Autoren postulierten, dass sich die Fläche der SN im Krankheitsverlauf nicht verändere und kein Maß für den Schweregrad der Erkrankung darstelle.

Allerdings hatte, wie auch in anderen Studien (BERG et al. 2001 a, WALTER et al. 2002), eine Minorität der DeNoPa-Patienten eine beidseits normale Echogenität. Möglicherweise könnte hierfür eine unerkannte MSA oder PSP verantwortlich sein. Die Verlaufsbeobachtung wird hier Aufschluss bringen.

Auch bei zwei der Kontrollpersonen (20\%) konnte eine Hyperechogenität nachgewiesen werden. Entsprechend der Annahme, die veränderte Echogenität sei ein Zeichen für die erhöhte Vulnerabilität der SN, hätten diese Personen ein erhöhtes Risiko im Laufe ihres Lebens ein IPS zu entwickeln. Die Kontrollperson mit der beidseitig erhöhten Echogenität wies zudem als einzige in der Kontrollgruppe eine milde Hyposmie im Riechtest auf. Möglicherweise handelt es sich hierbei bereits um eine nicht-motorische Frühmanifestation des IPS.

Da für die Schätzung des Anteils hyperechogener SN in der Normalbevölkerung bei BERG et al. (1999 a) nur die Personen mit deutlichem Ausprägungsgrad $\left(\geq 0,25 \mathrm{~cm}^{2}\right)$ gezählt wurden, lag der Anteil in der hier untersuchten Kontrollgruppe, trotz der kleinen Gruppenstärke, in einem vergleichbaren Bereich (BERG et al. 1999 a: 8,6 \%; DeNoPa: $10 \%$ ). Die mediane Fläche der SN von $0,09 \mathrm{~cm}^{2}$ in der Kontrollgruppe entsprach etwa den für diese Altersgruppe publizierten Daten. Die Mediane lagen bei BERG et al. (1999 a) bei $0,14 \mathrm{~cm}^{2}$ für 50-59-jährige, bei $0,10 \mathrm{~cm}^{2}$ für 60 -69-jährige und bei $0,15 \mathrm{~cm}^{2}$ für 70-79-jährige Personen.

Im DeNoPa-Kollektiv war bei $10 \%$ der Untersuchten entweder beidseits (2/40) oder einseitig (2/40) kein ausreichendes Knochenfenster vorhanden, was eine gewisse methodische Limitation darstellt. Dieses Problem wurde bereits bei WALTER et al. (2004) und BERG et al. (1999 a) beschrieben.

WALTER et al. (2004) regten an, das Ultraschallmerkmal der Hyperechogenität als Eingangskriterium für Studien $z u$ etablieren, um so die Homogenität von 
Studienpopulationen zu gewährleisten. Dies wurde in der hier vorliegenden Studie nicht umgesetzt. Folglich kann untersucht werden, ob die Fläche der SN bei Patienten mit normaler Echogenität langfristig zunimmt, ob sich Patienten mit normaler SN im Verlauf von solchen mit Hyperechogenität unterscheiden und ob es tatsächlich Patienten mit (autoptisch gesichertem) IPS und normaler Echogenität gibt oder ob diese Konstellation immer auf einer Fehldiagnose beruht. Die geplante Autopsie bei allen Studienteilnehmern im Rahmen der DeNoPa-Kassel-Studie kann auch Aufschluss darüber geben, ob es sich bei den gesunden Personen mit hyperechogener SN um die gleichen Personen handelt, bei denen in Autopsiestudien inzidentelle Lewy-Körper gefunden wurden (GIBB und LEES 1988), da es sich in beiden Fällen um Zeichen eines präsymptomatischen IPS handeln soll.

\subsection{Klinische Tests}

\subsubsection{Riechtest}

Die Auswertung des Riechtests bestätigte, dass Störungen der Geruchsfunktion ein häufiges Frühsymptom des IPS darstellen. $76 \%$ der getesteten De-novo-Parkinson-Patienten wiesen (basierend auf dem TDI-Score) mindestens eine Hyposmie auf, $28 \%$ eine funktionelle Anosmie. Andererseits hatten fast ein Viertel der Patienten einen normalen TDI-Score. Auch die Hyposmie war zu 50 \% nur mild ausgeprägt. Die Häufigkeit von Riechstörungen in der Patientengruppe unterschied sich damit nicht wesentlich von der Häufigkeit der motorischen Kardinalsymptome des IPS Rigor oder Ruhetremor, welche jeweils bei $73 \%$ der Patienten vorlagen. Die Sensitivität des TDI-Scores zur Unterscheidung zwischen Patienten und Kontrollpersonen lag bei $76 \%$, die Spezifität bei $88 \%$. Legt man die geltenden Kriterien für Biomarker beim M. Alzheimer zu Grunde (THE RONALD AND NANCY REAGAN RESEARCH INSTITUTE OF THE ALZHEIMER'S ASSOCIATION AND NATIONAL INSTITUTE ON AGING WORKING GROUP 1998), so wurde bei ausreichender Spezifität die geforderte Sensitivität von mindestens $80 \%$ nur knapp verfehlt. Da die Diagnose IPS bei allen Patienten entsprechend der international anerkannten UKPDSBB-Kriterien gestellt wurde und trotzdem etwa $25 \%$ eine Normosmie zeigten, erscheint es sinnvoll, dass Kriterium „Riechstörung“ als die Diagnose unterstützend und nicht als Ausschlusskriterium zu verwenden. Differentialdiagnostisch muss aber grundsätzlich auch an Erkrankungen wie z. B. MSA oder PSP gedacht werden, bei denen bisher keine oder nur geringgradige Beeinträchtigungen des Geruchssinns festgestellt wurden (WENNING et al. 1995). MÜLLER et al. (2002) untersuchten fünf De-novo-Patienten, von denen einer eine Normosmie aufwies. Da dieser außerdem kein Ansprechen auf die Parkinson-Behandlung zeigte, vermuteten die 
Autoren, dass eine Fehldiagnose vorläge. Im Gegensatz dazu hatten hier fünf der sieben Patienten mit Normosmie einen positiven L-Dopa-Test, was die Diagnose IPS bestätigt.

Die beobachteten Einschränkungen der Patienten bei Identifikation und Diskrimination stehen in Übereinstimmung mit anderen Untersuchungen (TISSINGH et al. 2001). Anders als in der genannten Studie oder bei DAUM et al. (2000) zeigte sich aber bei der Schwellenwertbestimmung trotz der großen Differenz der Mediane kein signifikanter Unterschied $(p>.05)$ zwischen den beiden Gruppen. Dies scheint nicht durch die Einnahme von Antiparkinsonmitteln zum Zeitpunkt des Tests begründet zu sein, da TISSING et al. die Signifikanz sowohl bei frühen unbehandelten als auch bei behandelten Patienten nachwiesen. Die klinischen Daten (UPDRS, H\&Y-Stadium) der unbehandelten Patienten unterschieden sich nur wenig von denen der DeNoPa-Gruppe.

Die Schwellenwerte der Kontrollgruppe waren vergleichbar mit denen, die von KOBAL et al. (2000) für gesunde Personen im Alter von über 55 Jahre ermittelt wurden (Median: 7,6, Range: 2-12,5), sodass die fehlende Signifikanz auch nicht durch unterdurchschnittliche Ergebnisse in der Kontrollgruppe erklärt werden kann. Was die Rauchgewohnheiten angeht, so gaben vier Patienten, aber keine Kontrollperson, an, aktuell zu rauchen. Da das Rauchen einen negativen Einfluss auf die Riechschwelle hat (KATOTOMICHELAKIS et al. 2007), ist dieser Faktor also nicht für die fehlende Signifikanz verantwortlich zu machen. Die Ursache könnte in den großen interindividuellen Unterschieden zwischen den Riechschwellen bei gleichzeitig relativ kleiner Gruppengröße zu suchen sein.

\subsubsection{Schellongtest}

Die Prävalenz der OH lag sowohl in der Gruppe der De-novo-Parkinson-Patienten als auch in der Kontrollgruppe bei $20 \%$, sodass sich kein signifikanter Unterschied fand. BONUCELLI et al. (2003) stellten hingegen in ihrer Studie mit 51 unbehandelten Patienten eine signifikante Abnahme der Blutdruckwerte im Vergleich zu gesunden Kontrollpersonen und eine Prävalenz der $\mathrm{OH}$ von $14 \%$ fest. Allerdings lagen dieser Auswertung nur jeweils zwei systolische Messergebnisse (vor dem Aufstehen und drei Minuten danach) zu Grunde. Die Definition der American Autonomic Society und American Academy of Neurology fordert hingegen die Berücksichtigung der systolischen und diastolischen Werte. Die Erhebungsmodalitäten, inklusive der Zahl der durchzuführenden Messungen innerhalb des 3-minütigen Zeitraums nach dem Aufstehen, werden dort allerdings nicht konkretisiert. Wäre wie bei BONUCELLI et al. (2003) vorgegangen worden, so wäre ebenfalls bei $14 \%(4 / 29)$ der DeNoPa-Patienten und bei keiner Kontrollperson eine $\mathrm{OH}$ diagnostiziert worden, und der Unterschied in der Prävalenz wäre wahrscheinlich signifikant ausgefallen. AWERBUCH und SANDYK (1992) ermittelten anhand des Kriteriums systolischer Druckabfall über 20 mmHg 
nach drei Minuten im Stehen eine ähnliche Prävalenz von $10 \%$ bei unbehandelten Patienten.

Die Häufigkeit der $\mathrm{OH}$ in beiden DeNoPa-Gruppen entsprach in etwa dem Auftreten in der amerikanischen Allgemeinbevölkerung mit einem Alter ab 65 Jahren (RUTAN et al. 1992). Insgesamt leiden dort $18 \%$ unter $\mathrm{OH}$, wobei die Werte je nach Klinik zwischen 10 und $26 \%$ schwanken. Allerdings lagen wiederum Differenzen im Vorgehen vor. Der Blutdruck wurde nur zu zwei Messzeitpunkten bestimmt. Auch andere Studien in verschiedenen Ländern machten Angaben zwischen 5 und $30 \%$ für ältere Personen (LOW 2008). Diese Übereinstimmung mit der Häufigkeit in der Allgemeinbevölkerung, zusammen gesehen mit der fehlenden Signifikanz im Gruppenvergleich, spricht eher für die $\mathrm{OH}$ als ein Phänomen des allgemeinen Alterns, als dafür, dass sie ein typisches Frühsymptom des IPS sei. Diese These schließt nicht aus, dass die Prävalenz der $\mathrm{OH}$ im Krankheitsverlauf über die in der älteren Allgemeinbevölkerung ansteigt, sei es durch das Voranschreiten pathologischer Veränderungen oder durch die Einnahme von L-Dopa oder Dopaminagonisten, deren Einfluss auf die OH kontrovers diskutiert wird (CALNE et al. 1970, SACHS et al. 1985, KUJAWA et al. 2000). In verschiedenen größeren Studien lag die Gesamtprävalenz der $\mathrm{OH}$ bei IPS zwischen 30 und 60 \% (GOLDSTEIN 2006) und damit über der bei unbehandelten Patienten. Die Untersuchung der kompletten DeNoPa-Studiengruppe im Längsschnitt kann hier weitere Erkenntnisse bringen. Zusätzlich stellt das Ergebnis die Tauglichkeit der frühen autonomen Dysfunktion als Kriterium in der Unterscheidung zwischen IPS und MSA in Frage. Es konnte gezeigt werden, dass eine $\mathrm{OH}$ auch bei IPS bald nach der Diagnosestellung vorhanden sein kann.

Der Vergleich der Ergebnisse verschiedener Studien zur Prävalenz der $\mathrm{OH}$ wurde dadurch erschwert, dass keine einheitlichen Kriterien (Berücksichtigung des diastolischen/ systolischen Blutdrucks, der Herzfrequenz und der Symptomatik, Grenzwerte) und Messmethoden (Ausgangsposition, Liegezeit vor dem Aufstehen, Anzahl und Zeitpunkte der Messungen im Stehen) verwendet wurden. Diese Problematik kann ursächlich für diskrepante Ergebnisse sein. Außerdem sind diverse Faktoren bekannt, die die Messung beeinflussen können: die Tageszeit, die Temperatur oder der Hydratationsstatus des Untersuchten sowie die Medikamenten-Einnahme (z. B. Diuretika, Vasodilatatoren, trizyklische Antidepressiva) (ROBERTSON 2008).

\subsubsection{Polysomnographie}

Patienten und Kontrollpersonen unterschieden sich nicht signifikant bezüglich des Vorliegens von RWA und RBD. Der Vergleich mit anderen Studien zeigte, dass der Grund hierfür eher im unerwartet hohen Anteil REM-Schlaf-assoziierter Veränderungen in der Kontrollgruppe zu 
suchen ist, zumal die prozentuale Häufigkeit der RBD bei den Patienten hier sogar über der in anderen Untersuchungen lag. In beiden Gruppen hatten ähnlich viele (50 bzw. $60 \%$ ) Personen polysomnographische Auffälligkeiten. Deutlicher unterschied sich der Anteil derer, die entweder eine RBD oder nur einen RWA aufwiesen. In anderen Studien lag der Anteil von Parkinson-Patienten mit RBD zwischen 15 und 50 \% (GJERSTAD et al. 2008, GAGNON et al. 2002, EISENSEHR et al. 2001, WETTER et al. 2000). Wenn die Diagnose nur mit Hilfe von Fragebögen oder Interviews gestellt wurde, waren die Prozentzahlen allgemein niedriger als bei polysomnographischen Untersuchungen, wahrscheinlich weil die RBD je nach Schweregrad nicht immer von den Betroffenen selbst wahrgenommen wird. Die höchste Prävalenz für die RBD bei IPS wurde von EISENSEHR et al. (2001) mit $47 \%$ angegeben. Allerdings wurden dort die Daten von Patienten untersucht, die zur Abklärung einer manifesten Schlafstörung eine Polysomnographie bekommen hatten. Die Häufigkeit von RBD-Diagnose in der vorliegenden Arbeit entsprach etwa der von GAGNON et al. (2002) für den RWA gefundenen Häufigkeit von $58 \%$. Wenn man annimmt, dass die RBD nicht in jeder Nacht zu beobachten ist, in der ein RWA gemessen werden kann, kann die Zahl der RBDBefunde nach zwei Nächten im Schlaflabor höher liegen. Allerdings tritt bei milden Formen das auffällige Verhalten im Schlaf unter Umständen seltener als ein Mal pro Monat auf (OLSON et al. 2000).

GJERSTAD et al. fanden 2008 eine signifikante Beziehung zwischen wahrscheinlicher RBD und niedrigerem UPDRS-III-Score. OLSON et al. (2000) und BOEVE (2004) postulierten, die Häufigkeit (und Schwere) der nächtlichen Aktivitäten im Sinne einer RBD könnte mit der Zeit im Rahmen der Neurodegeneration abnehmen. Diese Annahme könnte die hohe Prävalenz in dieser Untersuchung erklären, da es sich in den anderen oben erwähnten Studien überwiegend um Patienten mit länger andauernder Parkinson-Krankheit und nicht um Denovo-Patienten handelte. Allerdings lag die Häufigkeit in zwei kleinen Untersuchungen unbehandelter Patienten mit gering- bis mittelgradiger Erkrankung auch nur bei 12,5 (1/8) bzw. $30 \%$ (3/10), wobei in beiden Fällen nur eine Nacht im Schlaflabor ausgewertet wurde (WETTER et al. 1999 und 2000). Ob die dopaminerge Therapie bei IPS zu einem Anstieg oder Abfall der Frequenz und Schwere einer RBD führt ist umstritten. Beides wurde schon berichtet (GJERSTAD et al. 2008, OLSON et al. 2000). Dass keiner der DeNoPa-Patienten dopaminerge Medikamente einnahm und trotzdem $60 \%$ eine RBD hatten, spricht eher für einen positiven Effekt der Parkinson-Therapie auf die RBD.

Von den 50 \% der Kontrollpersonen mit RWA hatten wiederum 2/3 eine RBD. Ein denkbarer Grund für den hohen Anteil an Kontrollpersonen mit auffälligem REM-Schlaf ist die Erwähnung der zwei Nächte im Schlaflabor in dem Zeitungsartikel sowie im sonstigen Werbematerial für die Teilnahme an der Studie als Kontrollperson. Möglicherweise wurden 
dadurch gerade Personen (oder deren Angehörige) angesprochen, deren Schlaf in der Vergangenheit gestört war. Dies kann allerdings nur für die RBD, nicht für den RWA gelten. Eine andere Ursache könnte die kleine Gruppengröße sein. Trotzdem erscheint die Zahl sehr hoch, da die Prävalenz der RBD in der Gesamtbevölkerung auf unter $1 \%$ geschätzt wurde (OHAYON et al. 1997). Eine denkbare Erklärung wäre, dass es sich bei der RBD um ein generelles Alterungsphänomen handelt und die Häufigkeit der RBD in der älteren Allgemeinbevölkerung zunimmt.

Da die RBD-Diagnosen bei gleichzeitiger Betrachtung der Videoaufnahmen und polysomnographischen Messdaten gestellt wurden, erscheint es unwahrscheinlich, dass Differenzialdiagnosen der RBD für die hohen Prozentzahlen verantwortlich sind. Somnambulismus zum Beispiel ist eine Non-REM-Parasomnie, eine obstruktive Schlafapnoe kann sich zwar ähnlich wie eine RBD manifestieren, ist aber nicht mit einem RWA assoziiert und epileptische Anfälle wären gegebenenfalls in der EEG detektiert worden.

In der hier vorliegenden Untersuchung wies also ein großer Anteil von frühen, unbehandelten Parkinson-Patienten eine RBD auf. Dies unterstützt die Vorstellung, dass es sich bei der RBD um eine Frühmanifestation des IPS handelt. Allerdings erscheint dies, mit Blick auf den nicht signifikant niedrigeren Anteil in der Kontrollgruppe, nicht ausreichend zu sein, um als diagnostisches Kriterium eingesetzt zu werden. Es bleibt abzuwarten, ob sich in der Hauptstudie mit einer größeren Zahl von Studienteilnehmern klarere Unterschiede feststellen lassen.

Bei Betrachtung der Verteilung der maximalen RBD-Grade in den beiden Gruppen fiel auf, dass alle Kontrollpersonen, die nicht nur einen RWA hatten (entspricht Grad 0.0), mindestens Bewegungen der proximalen Extremitäten zeigten, während bei der Hälfte der Patienten im Video ausschließlich Bewegungen der distalen Extremitäten oder des Gesichts mit oder ohne Vokalisationen beobachtet wurden. Bei einem Patienten konnten sogar nur Vokalisationen aufgezeichnet werden (Grad 0.1). Die andere Hälfte der Patienten zeigte, wie die Kontrollpersonen, ausgedehntere Bewegungen. Ähnliches beschrieben EISENSEHR et al. (2001). Sie beobachteten, dass der klinische Ausdruck der RBD bei IPS-Patienten stärker variierte als bei idiopathischer RBD. Die idiopathische RBD äußerte sich durch schwerere Verhaltensstörungen im REM-Schlaf. Es gab keine milden Formen, wie bei ParkinsonPatienten mit RBD. Das Vorhandensein geringerer Ausprägungsgrade scheint aber nicht, wie von EISENSEHR et al. (2001) vermutet, mit der dopaminergen Medikation in Zusammenhang zu stehen, da die DeNoPa-Patienten alle unbehandelt waren.

Allerdings stellt sich auch die Frage, ab wann eine motorische Aktivität während des REMSchlafes im Sinne einer RBD interpretiert werden kann und sollte. Die ICSD-Kriterien sprechen von exzessiven Extremitäten- oder Körperbewegungen, komplexem, lebhaftem oder potenziell verletzendem Verhalten, aber diese Aktivitäten sind nicht genauer definiert 
und können sehr unterschiedlich interpretiert werden. In einem Polysomnographie-Befund wurde z. B. eine „Episode mit kurzem Zucken des rechten Arms, Lachen und Grimassieren“ beschrieben. In drei anderen Berichten war von „kurzen Kaubewegungen“ und „wiederholten Kopfbewegungen“ die Rede. Ein RWA als Voraussetzung für das Vorliegen einer RBD war bei allen gegeben. Diese Befunde wurden hier als milde RBD vom Schweregrad 1.0 bzw. 1.1 gewertet. Wären diese grenzwertigen Befunde bei den drei Patienten nicht als Zeichen einer RBD eingestuft worden, so wären sich die beiden untersuchten Gruppen noch ähnlicher bezüglich Veränderungen des REM-Schlafs gewesen.

\subsubsection{Neuropsychologische Tests}

Betrachtet man die Ergebnisse des neuropsychologischen Screenings, so scheint es, dass sich der kognitive Status bei den frühen, unbehandelten Parkinson-Patienten nicht von dem altersentsprechenden Zustand bei Personen ohne IPS unterscheidet. Sowohl im MMST als auch im Uhrentest zeigte der Gruppenvergleich keine signifikante Ungleichheit. Dies wirkt unvereinbar mit den Ergebnissen anderer Studien: Bei MUSLIMOVI et al. (2005) schnitten 115 neu diagnostizierte Patienten in 20 von 28 neuropsychologischen Test schlechter ab als 70 gesunde Kontrollpersonen. Besonders häufig waren Einschränkungen in den Bereichen Exekutivfunktionen und Gedächtnis. $24 \%$ der Patienten wurden als kognitiv beeinträchtigt eingeschätzt. In einer Untersuchung von AARSLAND et al. (2009 b) hatten $19 \%$ von 196 frühen, unbehandelten IPS-Patienten eine leichte kognitive Beeinträchtigung, ein sogenanntes $\mathrm{MCl}$ (engl. minimal cognitive impairment). Geringe Einschränkungen waren in allen kognitiven Tests vorhanden, besonders beim verbalen Gedächtnis. Auch ELGH et al. (2009) beschrieben ein signifikant schlechteres Abschneiden von 88 neu diagnostizierten Patienten in diversen Aufgaben. $30 \%$ hatten Defizite in mindestens einer der Domänen episodisches Gedächtnis, Arbeitsgedächtnis, visuell-räumliche Fähigkeiten, Wortflüssigkeit, Benennung oder exekutive Funktionen. Nur bei AARSLAND et al. (2009 b) unterschieden sich allerdings die Patienten signifikant im MMST von den gesunden Kontrollpersonen. In den beiden anderen Studien war dies, wie in der DeNoPa-Pilotstudie, nicht der Fall.

Die DeNoPa-Patienten schnitten beim Vergleich der Mittelwerte der vier Studien am besten ab (siehe Tabelle 4-1), obwohl in den anderen Studien Demenz zu den Ausschlusskriterien gehörte und ein DeNoPa-Patient mit einem Score von 21 die von DUBOIS et al. (2007 a) vorgeschlagene Grenze von 26 Punkten zur Identifikation einer kognitiven Einschränkung bei Parkinson unterschritt. Das mittlere Alter der Patienten differierte zwischen den Studien nur um weniger als zwei Jahre, wobei es bei ELGH et al. (2009) am höchsten lag. Die Bildungsjahre als weiterer Einflussfaktor ließen sich nicht vergleichen, da diese in den zum Vergleich herangezogenen Studien wahrscheinlich nicht nur die Schulzeit, sondern auch die 
Ausbildungsjahre beinhalteten. Da in den drei zum Vergleich herangezogenen Studien nur jeweils 20 bis $30 \%$ der Patienten (unterschiedlich definierte) kognitive Beeinträchtigungen aufwiesen, ist es denkbar, dass nur wenige der DeNoPa-Patienten kognitive Defizite hatten. Dieser Umstand könnte die verhältnismäßig hohen mittleren MMST-Ergebnisse und die fehlende Signifikanz im Gruppenvergleich erklären.

Tabelle 4-1: Mittelwerte des Mini-Mental-Status-Tests verschiedener Untersuchungen an neu diagnostizierten Parkinson-Patienten und gesunden Kontrollpersonen

\begin{tabular}{|l|l|l|l|l|}
\hline & $\begin{array}{l}\text { MUSLIMOVI } \\
\text { et al. 2005 }\end{array}$ & $\begin{array}{l}\text { AARSLAND et al. } \\
2009 \mathrm{~b}\end{array}$ & $\begin{array}{l}\text { ELGH et al. } \\
2009\end{array}$ & $\begin{array}{l}\text { DeNoPa- } \\
\text { Pilotstudie }\end{array}$ \\
\hline Patienten & $\begin{array}{l}27,9 \\
(\mathrm{~N}=115)\end{array}$ & $\begin{array}{l}27,9 \\
(\mathrm{~N}=196)\end{array}$ & $\begin{array}{l}28,7 \\
(\mathrm{~N}=88)\end{array}$ & $\begin{array}{l}28,9 \\
(\mathrm{~N}=30)\end{array}$ \\
\hline $\begin{array}{l}\text { Kontroll- } \\
\text { personen }\end{array}$ & $\begin{array}{l}28,3 \\
(\mathrm{~N}=70)\end{array}$ & $\begin{array}{l}28,6 \\
(\mathrm{~N}=171)\end{array}$ & $\begin{array}{l}29,1 \\
(\mathrm{~N}=30)\end{array}$ & $\begin{array}{l}29,1 \\
(\mathrm{~N}=10)\end{array}$ \\
\hline $\begin{array}{l}\text { p-Wert } \\
\text { (Signifikanzwert) }\end{array}$ & $>.05$ & $<.001$ & $>.05$ & $>.05$ \\
\hline
\end{tabular}

(N: Stichprobenumfang)

Ein weiterer Grund für die unterschiedlichen Studienergebnisse könnte in der verglichen mit anderen Methoden verhältnismäßig niedrigen Interrater-Reliabilität des MMST liegen (MOLLOY et al. 1991). Da die Benutzungsrichtlinien relativ kurz sind und es auch kein Zeitlimit gibt, kann die Anwendung und Punktebewertung zwischen verschiedenen Beurteilern variieren.

Die mittleren Scores der DeNoPa-Kontrollpersonen und der Kontrollpersonen in der Studie von ELGH et al. (2009) lagen mit 29,1 am nächsten an der altersentsprechenden Norm von 29, die von CRUM et al. (1993) bei einer bevölkerungsbasierten Untersuchung mit über 18.000 Teilnehmern für Personen im Alter zwischen 60 und 70 Jahren ermittelt wurde.

Dass in den drei oben genannten Studien bei der ausführlichen neuropsychologischen Testung deutliche Gruppenunterschiede festzustellen waren und der MMST trotzdem in zwei der Studien keinen signifikanten Unterschied zeigte, spricht für eine limitierte Aussagekraft des MMST im frühen Stadium des IPS bei subtilen Veränderungen der kognitiven Fähigkeiten, zumal 1/3 der Punkte alleine für zeitliche und örtliche Orientierung vergeben werden, die aber beim IPS eher nicht beeinträchtigt sind (BRONNICK et al. 2007). Dies geht konform mit dem Ergebnis von MAMIKONYAN et al. (2009), das eine intakte globale Kognition, gemessen durch den MMST, kognitive Einschränkungen nicht ausschließt. Auch RIEDEL et al. (2008) stellten die Eignung des MMST bei IPS in Frage, da dieser in der untersuchten Patientengruppe nur eine Prävalenz von 18 \% für kognitive Einschränkungen ergab, obwohl $29 \%$ der Patienten klinische Kriterien für eine Demenz erfüllten. Allerdings 
handelt es sich beim MMST auch ausdrücklich um ein Screening-Instrument, das keine ausführliche Testung ersetzt (FOLSTEIN 1975).

Bei Parkinson-Demenz sind neben den kognitiven Bereichen Aufmerksamkeit, Gedächtnis und Exekutivfunktionen auch die visuell-konstruktiven Fähigkeiten eingeschränkt (EMRE et al. 2007). Diese werden typischerweise mit Hilfe von Zeichentests wie dem von SHULMAN KI et al. (1986) entwickelten Uhrentest beurteilt. Defizite der visuell-räumlichen Wahrnehmung und konstruktive Fähigkeiten unter visueller Kontrolle können bereits bei Patienten ohne Demenz nach kurzem Verlauf der Parkinson-Krankheit auftreten: Ein Uhrentest gehörte bei MUSLIMOVIĆ et al. (2005) zu den Tests mit der höchsten Rate an Einschränkungen. Diese Schwierigkeiten wurden eher Einschränkungen in den exekutiven Funktionen zugeschrieben als einem unabhängigen Defizit der visuell-konstruktiven Fähigkeiten. Die Ergebnisse der DeNoPa-Patienten beim Uhrentest unterschieden sich allerdings nicht signifikant von denen der Kontrollpersonen. Der Uhrentest ergab eine Prävalenz kognitiver Einschränkungen in der Patientengruppe von $27 \%$. Verglichen mit den Häufigkeiten von Scores über dem Cut-off-Wert bei RIEDEL et al. (2008) für IPS-Patienten mit vergleichbarem Krankheitsstadium (Stadium I: 30,4 \%; Stadium II: 34,1 \%) oder Alter (s 65 Jahre: 24,4 \%; 66-70 Jahre: 40,4\%), hatten die DeNoPa-Patienten erwartungsgemäße Ergebnisse.

Die Prävalenz von Demenz bei Personen im Alter von 65-69 Jahren in Deutschland liegt unter 1,5\% (ZIEGLER und DOBLHAMMER 2009). Auch wenn Schwierigkeiten im Uhrentest nicht gleichzusetzten sind mit Demenz, so erscheint der Anteil der Kontrollpersonen mit Ergebnissen über dem Cut-off-Wert mit $22 \%$ relativ hoch. Allerdings kann bei einer kleinen Kontrollgruppe das Bild leicht durch wenige unterdurchschnittliche Ergebnisse verzerrt werden, sodass die fehlende Signifikanz nicht grundsätzlich ausschließt, dass Patienten mit frühem IPS häufiger eingeschränkt sind als altersentsprechende Kontrollpersonen.

\subsection{Fragebögen}

\subsubsection{NMSQuest}

Die Auswertung des NMSQuest zeigte, dass NMS bereits bei Parkinson-Patienten im frühen Krankheitsstadium signifikant häufiger anzutreffen sind als bei gleichaltrigen Kontrollpersonen. Dieses Ergebnis bekommt eine besondere Relevanz vor dem Hintergrund, dass NMS einen großen Einfluss auf die Lebensqualität haben (RAHMAN et al. 2008), aber häufig unerkannt und damit unbehandelt bleiben (SHULMAN LM et al. 2002).

Die Bandbreite der berichteten Symptome war angesichts des frühen Krankheitsstadiums sehr groß und interindividuell unterschiedlich: Von den 30 abgefragten Symptomen waren 27 
bei mindestens einem Patienten im letzten Monat aufgetreten und jeder Patient hatte durchschnittlich 7 NMS.

In der ersten internationalen Studie mit dem NMSQuest zur Prävalenz von NMS unter Parkinson-Patienten aller Krankheitsstadien lag der Durchschnitt bei zehn (MARTINEZMARTIN et al. 2007). Die Prävalenz von NMS stieg dort allerdings mit steigendem Schweregrad und zunehmender Erkrankungsdauer. Die Patienten mit mildem Schweregrad (H\&Y-Stadium 1-2,5) oder kurzer Erkrankungsdauer (< 5 Jahre) hatten im Mittel 8,8 bzw. 8,5 NMS, was 27 bzw. 29 \% Ja-Antworten entspricht. In der Pilotstudie der NMSQuest Study von CHAUDHURI et al. (2006 b) hatten die Patienten mit milder Erkrankung (Stadium 1-2) durchschnittlich 8,0 NMS. Diese Untergruppen ähneln in ihrer Zusammensetzung am ehesten der DeNoPa-Gruppe und zeigen ein vergleichbares Ergebnis.

Die beiden häufigsten Symptome der DeNoPa-Patienten waren Harndrang und Nykturie. Urologische Symptome treten also entgegen anders lautenden Ansichten (STOCCHI et al. 1997) auch beim IPS im frühen Stadium häufiger auf als bei nach Alter und Geschlecht gematchten gesunden Personen (69\% vs. $40 \%$ ). Die Prävalenz von Harndrang und Nykturie überstieg in der DeNoPa-Patientengruppe die in anderen Studien: MARTINEZMARTIN et al. (2007) ermittelten für Harndrang und Nykturie eine Häufigkeit von 56 \% bzw. $62 \%$ (mittleres H\&Y-Stadium: 2,5, mittleres Patientenalter: 68 Jahre). Eine Fragebogenerhebung zur Funktion der Beckenorgane von SAKAKIBARA et al. (2010) ergab bei $42 \%$ der weiblichen und $54 \%$ der männlichen Patienten einen verstärkten Harndrang und bei $53 \%$ der weiblichen und $63 \%$ der männlichen Patienten eine Nykturie (mittleres H\&Y-Stadium: 3, mittleres Patientenalter: 59 Jahre). Laut dieser Untersuchung soll die Häufigkeit der Symptome mit zunehmendem Krankheitsstadium steigen, aber bei Patienten unabhängig vom Alter sein. Folglich können Alter und Stadium nicht die höhere Prävalenz bei den DeNoPa-Patienten erklären. Da die DeNoPa-Patienten unbehandelt waren, liegt die Vermutung nahe, die Parkinson-Therapie sei für die niedrigere Prävalenz bei behandelten Patienten verantwortlich. Frühere Studien zeigen allerdings widersprüchliche Ergebnisse, was den Effekt der dopaminergen Therapie auf die Miktion angeht (ARANDA und CRAMER 1993, BENSON et al. 1976, WINGE et al. 2004).

An zweiter und dritter Stelle der häufigsten Symptome bei den De-novo-Parkinson-Patienten standen Vergesslichkeit (52\%), Konzentrationsprobleme und Insomnie (jeweils $48 \%$ ). Bei MARTINEZ-MARTIN et al. (2007) folgten hingegen auf die urologischen Symptome zunächst Obstipation (52\%) und Depression (50\%). Erst an fünfter Stelle standen Konzentrationsschwierigkeiten und Insomnie (jeweils $46 \%$ ). Das Muster der vorherrschenden NMS scheint sich also im Krankheitsverlauf zu verändern. Möglicherweise sind aber nicht nur das Krankheitsstadium oder die Erkrankungsdauer, sondern auch kulturelle Unterschiede zwischen den Studienpopulationen für die teilweise ungleiche 
Rangfolge ursächlich. Die Ergebnisse der zum Vergleich herangezogenen Studie wurden in sechs Ländern (Vereinigtes Königreich, USA, Deutschland, Israel, Japan und Italien) gewonnen, wohingegen die DeNoPa-Patienten alle aus Deutschland stammen. Ein Hinweis für derartige Einflüsse kommt von TSUBOI et al. (2006), die beim Vergleich der Prävalenz von NMS bei europäischen und japanischen Patienten herausfanden, dass letztere beispielsweise signifikant häufiger Obstipation oder Probleme beim Schlucken hatten.

Das Muster der aufgetretenen Symptome zeigte Übereinstimmung mit den Beobachtungen von CLAYTON et al. (2006) bei unbehandelten Parkinson-Patienten. In beiden Studien hatten über $30 \%$ der Patienten Probleme mit Harndrang, Nykturie, Vergesslichkeit, Interessenverlust, Konzentration, Depression und Insomnie. Die Prävalenz der Depression (34 \%) war bei den DeNoPa-Patienten zwar geringer als in der heterogenen Patientengruppe von MARTINEZ-MARTIN et al. (2007), entsprach aber der von AARSLAND et al. (2009 a) für frühe, unbehandelte Parkinson-Patienten ermittelten Häufigkeit. Laut CHAUDHURI et al. (2006 a) besteht überzeugende Evidenz dafür, dass Depression zu den Charakteristika des IPS im prämotorischen Stadium zählt. Es wurde mehrfach eine signifikante positive Assoziation zwischen einer Depression in der Vorgeschichte und der Wahrscheinlichkeit, an IPS zu erkranken, gemessen (ISHIHARA und BRAYNE 2006). Passend zu diesem Ergebnis hatte keine der gesunden Kontrollperson depressive Symptome. Da die depressiven Symptome bei einem Teil der neu diagnostizierten Patienten im Verlauf wieder zurückgehen, scheinen nicht nur neurodegenerative Veränderungen im Gehirn der Patienten, sondern auch eine reaktive Komponente (Konfrontation mit der Diagnose einer chronischenprogredienten, nicht heilbaren Krankheit) an der Entstehung der Depression beteiligt zu sein (RAVINA et al. 2009).

Wahnvorstellungen und Halluzinationen, die bei keinem der DeNoPa-Patienten vorkamen, waren hingegen bei 11 bzw. $23 \%$ der Patienten von MARTINEZ-MARTIN et al. (2007) vorhanden. Dies deckt sich mit Studienergebnissen, die zeigen, dass die Prävalenz klinisch signifikanter psychotischer Symptome bei frühen unbehandelten Patienten unter $1 \%$ (AARSLAND et al. 2009 a) liegt und im Erkrankungsverlauf deutlich steigt (FORSAA et al. 2010, GOETZ et al. 2010).

Auffällig war, dass Veränderungen von Geschmack oder Geruch bei $45 \%$ der Patienten auftraten, aber von keiner gesunden Person bejaht wurden. Dies unterstreicht die Annahme der Riechstörung als typisches Frühsymptom des IPS und deckt sich dahingehend mit den Ergebnissen des Riechtests, dass dort ein signifikant schlechteres Riechvermögen bei Patienten festgestellt wurde. Die Selbsteinschätzung der Patienten wies aber eine starke Diskrepanz zu den Resultaten der Riechprüfung auf, in der nicht $45 \%$ sondern insgesamt $87 \%$ der Studienteilnehmer ein Defizit aufwiesen. Eine derartige Beobachtung wurde auch von DAUM et al. (2000) gemacht. 


\subsubsection{NMSScale}

Die weiterführende Beurteilung von Schwere und Häufigkeit der NMS ergab einen signifikanten Unterschied zwischen Patienten- und Kontrollgruppe, wie die Ergebnisse des NMSQuest erwarten ließen. Die mittlere Punktzahl lag bei den Patienten bei unter $10 \%$ der theoretisch möglichen Maximalpunktzahl, sodass der Ausprägungsgrad der NMS bei frühen Parkinson-Patienten insgesamt als mild gelten kann.

Da sowohl Prävalenz als auch Schwere der NMS im Laufe des Krankheitsprogresses steigen (MARTINEZ-MARTIN et al. 2009), war absehbar, dass mittlere und mediane Gesamtpunktzahl bei den DeNoPa-Patienten niedriger lagen als in der internationalen Validierungs-Studie der NMSScale (ebd.), in der Patienten aller Krankheitsstadien mit deutlich längerer mittlerer Krankheitsdauer (8,1 $\pm 5,7$ Jahre) eingeschlossen waren (Median: 43 Punkte, Mittelwert: 57 Punkte). Entsprechend lag auch der Anteil der Patienten ohne NMS bei DeNoPa mit 7,7\% höher als in der zitierten Studie (0,5\%). In Patientengruppen mit Krankheitsdauer unter 5 Jahren oder H\&Y-Stadium 2 lagen die Mittelwerte mit 45,8 bzw. 49,1 Punkten näher an dem der DeNoPa-Patienten.

Die Symptom-Bereiche Miktion, Aufmerksamkeit/ Gedächtnis und Stimmung machten bei den DeNoPa-Patienten den größten Anteil an der Gesamtpunktzahl aus. Bei MARTINEZMARTIN et al. (2009) lag hingegen der Bereich Stimmung an erster Stelle, gefolgt von Schlaf und Miktion. Mögliche Gründe für diese Diskrepanz können auch hier neben Stadium und Dauer der Parkinson-Erkrankung länderspezifische Unterschiede oder die kleine Gruppengröße in der DeNoPa-Pilotstudie sein.

Insgesamt zeigen die Ergebnisse der NMSQuest und NMSScale, dass bei ParkinsonPatienten bereits nach kurzem Krankheitsverlauf eine Vielzahl von NMS vorhanden ist, die in ihrer Gesamtheit deutlich häufiger sind, als es dem Alter der Patienten entsprechen würde. Die Verteilung der NMS ist zudem individuell sehr unterschiedlich. Dies bestätigt die Darstellung von LANGSTON (2006), der die Parkinson-Krankheit mit einem Eisberg verglich, deren motorische Symptome, hervorgerufen durch die neuronale Degeneration in der SN, nur die Spitze darstellen. Unter der Wasseroberfläche würden sich aber, bedingt durch ausgedehnte Veränderungen im zentralen und peripheren Nervensystem, eine große Zahl weiterer klinischer Zeichen und Symptome verbergen.

Viele der Symptome sind relativ unspezifisch, sodass sie, besonders wenn sie das klinische Bild dominieren, Anlass für Fehldiagnosen sein können. Zum anderen werden NMS von den Patienten sicher oft nicht mit der „Schüttellähmung“ in Verbindung gebracht, sodass sie diese nicht von sich aus dem Arzt gegenüber erwähnen. Da die NMS erheblich zur 
Behinderung und Minderung der Lebensqualität der Patienten beitragen können, sollten ihre Erkennung und Therapie bereits zum Zeitpunkt der Diagnosestellung einen hohen Stellenwert in der Patientenversorgung einnehmen.

\subsubsection{PDQ-39}

Das IPS hat einen negativen Einfluss auf diverse Aspekte der Lebensqualität von Patienten (SCHRAG et al. 2000). Besondere Bedeutung kommt dabei den motorischen Problemen (Stürze, Gangstörungen, Schwierigkeiten beim Ankleiden), aber auch kognitivpsychiatrischen (Depression, Verwirrung) und autonomen Symptomen (Harninkontinenz) sowie Therapiekomplikationen (unvorhersehbare "On-off"-Fluktuationen) zu (RAHMAN et al. 2008).

MARTINEZ-MARTIN et al. (2009) stellten eine enge Assoziation zwischen den Ergebnissen von NMSScale und PDQ-39 fest. Dementsprechend war auch beim PDQ-39 der Unterschied in der Gesamtpunktzahl zwischen Patienten und Gesunden signifikant. Die Erkrankung scheint also schon im frühen Stadium deutliche funktionelle, psychische oder soziale Auswirkungen auf die Patienten zu haben.

Verglichen mit den Ergebnissen von SCHRAG et al. (2000) oder RAHMAN et al. (2008) lagen die SI-Werte der DeNoPa-Patienten niedriger, auch wenn die Daten von Patientengruppen mit ähnlichem Erkrankungsstadium zum Vergleich herangezogen wurden (Mittelwerte: 22,8 (H\&Y-Stadium 1,5), 23,9 (Stadium 2) bei SCHRAG et al. 2000 bzw. 25,2 (Stadium $\leq 2,5$ ) bei RAHMAN et al. 2008). Eine denkbare Erklärung hierfür kommt von HARIZ und FORSGREN (2011), die herausfanden, dass das Ausmaß an Einschränkungen, das die neu diagnostizierten Patienten erfahren, stark vom Subtyp der Erkrankung abhängt. Sie unterschieden einen posturale-Instabilität-und-Gangstörungen-Typ, einen Tremordominanten Typ und einen unklaren Typ, wobei die Auswirkungen auf die Lebensqualität bei ersterem am gravierendsten ausfielen. Daraus folgt, dass der Anteil von Patienten mit den verschiedenen Typen (besonders bei kleinen Studiengruppen) Einfluss auf die Ergebnisse beim PDQ-39 nehmen kann. Der mittlere SI lag beim posturale-Instabilität-undGangstörungen-Typ bei etwa 25, während er in den anderen Gruppen etwas über 15 betrug (ungefähre Angaben abgelesen aus Abbildung 2 von HARIZ und FORSGREN 2011). Es fand jedoch bei keiner der oben verglichenen Studien eine Unterscheidung von Erkrankungstypen statt, sodass der tatsächliche Einfluss dieses Faktors hier nicht überprüft werden kann.

Einer der Patienten erreichte bereits fast $50 \%$ der Maximalpunktzahl, während zwei Patienten einen SI von 0 hatten. Das Ausmaß der subjektiv empfundenen Einschränkungen ist also interindividuell sehr variabel. Überraschenderweise hatten alle drei Patienten ein 
Erkrankungsstadium von 2,5, obwohl sich bei FITZPATRICK et al. (1997) und SCHRAG et al. (2000) die Subscores aller einzelnen Bereiche des PDQ-39 (außer soziale Unterstützung und Stigma bei SCHRAG) und auch der PDQ-39SI signifikant mit steigendem Erkrankungsstadium verschlechterten.

Im Vergleich mit der Kontrollgruppe hatten die DeNoPa-Patienten in allen Punkten häufiger Einschränkungen. Die Hauptbeeinträchtigungen der frühen Parkinson-Patienten lagen in den Gebieten emotionales Wohlbefinden, körperliches Unbehagen, Kognition und Alltagsaktivitäten. Besonders viele Patienten hatten zumindest zeitweise emotionale Probleme wie Niedergeschlagenheit oder Zukunftsangst. (Milde) depressive Symptome treten auch laut RAVINA (2009) und AARSLAND (2009 a) bei neu-diagnostizierten Patienten häufig auf, und Depression ist als Hauptprädiktor für die Lebensqualität bei Parkinson bekannt (SCHRAG et al. 2000). Drei Viertel der Patienten und damit $55 \%$ mehr als in der Kontrollgruppe beklagten zudem ein schlechtes Gedächtnis. Diese subjektive Bewertung zeigt, dass sich die in neuropsychologischen Tests bei frühen, unbehandelten Patienten nachweisbaren Einschränkungen verschiedener Bereiche der Gedächtnisleistung (MUSLIMOVIC et al. 2005, COOPER et al. 1991, AARSLAND et al. 2009 b) bereits im Alltag der Patienten bemerkbar machen.

Der Unterschied in der Häufigkeit von Problemen war am größten bei der Frage nach der Ausübung von Freizeitaktivitäten: Schwierigkeiten kamen bereits bei fast $60 \%$ der Patienten zumindest „selten“ und bei fast $20 \%$ sogar "häufig“ vor, wurden aber von keiner Kontrollperson bejaht. Dies könnte je nach Art der Aktivität durch eine eingeschränkte Mobilität der Patienten bedingt sein, z. B. durch Angst in der Öffentlichkeit zu fallen. Die Furcht vor Stürzen hat laut BROZOVA et al. (2009) verglichen mit anderen Aspekten der Gangstörung den größten Einfluss auf die Lebensqualität. Ein anderer Gesichtspunkt könnte die Befürchtung einer Stigmatisierung sein. Dass eine solche existiert, zeigten MOORE und KNOWLES (2006) in einer Befragung zur öffentlichen Meinung über die ParkinsonKrankheit. Fast $70 \%$ der dort Befragten gaben an, sie würden im Falle einer Erkrankung nur einem begrenzten Personenkreis von ihrer Krankheit erzählen. Etwa 50 \% glaubten, dass es eine Stigmatisierung gebe. Nur die Hälfte der Personen wusste, dass die Erkrankung nur langsam voranschreitet und eine Demenz nicht zwangsläufig auftritt. Ein kleiner Teil der Befragten brachte die Erkrankung mit Nervosität oder mit Lebensstilfaktoren wie Rauchen oder großem Kaffeekonsum in Verbindung oder glaubte, es handele sich um eine Form der Geisteskrankheit. Daher ist es nicht überraschend, dass ein Drittel der DeNoPa-Patienten sich Sorgen über die Reaktion anderer Menschen gemacht hatte oder das Gefühl kannte, die Krankheit verheimlichen zu müssen.

Schwierigkeiten bei der Ausübung von Freizeitaktivitäten können auch Schmerzen oder nachlassende feinmotorische Fähigkeiten gemacht haben. Bereits James Parkinson 
berichtete von einer „...unsteadiness [...] whilst writing...” im frühen Verlauf der Erkrankung und einem Versagen der Hand „... to answer with exactness to the dictates of the will“ (PARKINSON 1817/ 2002, S. 224). Über $40 \%$ der Patienten hatten häufig oder immer Probleme deutlich zu schreiben. Diese Einschätzung der eigenen Fähigkeiten steht in Einklang mit Ergebnissen von PONSEN et al. (2008), die bei neu diagnostizierten, unbehandelten Patienten eine signifikant verminderte Schreibgeschwindigkeit und progressive Mikrographie neben anderen Einschränkungen komplexer Bewegungen der oberen Extremität feststellten.

Aus Patientenperspektive gab es also bereits deutliche Einschränkungen funktionaler Fähigkeiten (Mobilität und Ausübung von Alltagsaktivitäten) und damit des sozialen Lebens der Betroffenen sowie Auswirkungen auf die psychische und emotionale Gesundheit.

\subsubsection{RBDSQ}

Der RBDSQ zeigte einen signifikanten Unterschied im Gruppenvergleich. Die Patienten erreichten im Schnitt höhere Ergebnisse.

Die Frage nach Bewegungen der Arme oder Beine im Schlaf wurde am häufigsten bejaht. Diese ist aber relativ unspezifisch. Es könnte sich dabei neben der Manifestation einer RBD auch um Einschlafmyoklonien, Somnambulismus, motorische Unruhe bei obstruktiver Schlafapnoe, Restless-Legs-Syndrom oder periodische Beinbewegungen handeln. Die letzten drei treten bei Parkinson-Patienten signifikant häufiger auf als bei Gesunden (WETTER et al. 2000, NOMURA et al. 2006, KRISHNAN et al. 2003, HARDIE et al. 1986).

Die Fragen mit der höchsten Spezifität für die RBD laut STIASNY-KOLSTER et al. (2007) (6.3: komplexe Bewegungen; 6.4: umgefallene Gegenstände; 5: Verletzungen) wurden von keiner Kontrollperson und nur zwei von jeweils einem Patienten mit RBD bejaht.

Die Gesamtpunktzahlen in beiden DeNoPa-Gruppen waren verglichen mit denen in der eben genannten Studie insgesamt niedrig. Dort lag der mittlere RBDSQ-Score der Patienten mit polysomnographisch gesicherter RBD bei 9,5 $( \pm 2,8)$ Punkten, während die Kontrollgruppe mit ausgeschlossener RBD einen mittleren Score von 4,6 $( \pm 3,0)$ Punkten erreichte.

Obwohl bei über 60 \% der DeNoPa-Patienten im Schlaflabor eine RBD nachweisbar war, lag die Maximalpunktzahl im RBDSQ schon bei 6. Nur zwei Patienten hatten ein positives Testergebnis, also fünf oder mehr Punkte. Bei diesem Cut-off-Wert liegt laut STIASNYKOLSTER et al. (2007) die Sensitivität des Fragebogens zur Unterscheidung von Personen mit polysomnographisch gesicherter und ausgeschlossener RBD bei $96 \%$ und die Spezifität bei $56 \%$. Für die DeNoPa-Studienteilnehmer lag die Spezifität bei $100 \%$, während die Sensitivität nur $14 \%$ betrug. Es wurden also durch den Fragebogen nur etwa ein Sechstel 
der Studienteilnehmer mit RBD erkannt (18\% der Patienten und $0 \%$ der Kontrollpersonen mit RBD).

EISENSEHR et al. (2001), die bei Patienten mit Schlafstörungen Interviews und anschließend Polysomnographien durchführten, um die Sensitivität und Spezifität der klinischen RBD-Diagnose zu ermitteln, beschrieben eine ähnliches Phänomen. Sie fanden bei Parkinson-Patienten eine deutlich niedrigere Sensitivität der Interviews (33\%) als bei Patienten ohne IPS (100\%). Gleichzeitig beobachteten sie in Polysomnographiesynchronisierten Videos, dass die klinischen Äußerungen der RBD bei den IPS-Patienten interindividuell sehr stark variierten. Die Patienten mit idiopathischer RBD zeigten ein ausgeprägtes Agieren, während bei einem Teil der Patienten mit IPS und RBD nur subtile Zeichen wie kurze Extremitätenbewegungen oder Reden im Schlaf auf die RBD hinwiesen. EISENSEHR et al. (2001) schlossen, dass es diese milden Formen der RBD bei IPS seien, die im klinischen Interview leichter übersehen würden. Diese Schlussfolgerung könnte auch die niedrige Sensitivität des RBDSQ sowie die insgesamt niedrigen Punktzahlen bei der DeNoPa-Stichprobe erklären. Möglicherweise war die RBD bei den Patienten subklinisch, d. h. die nächtlichen Aktivitäten waren nicht so ausgeprägt, dass sie den Patienten selber oder deren Partnern (wenn vorhanden) aufgefallen wären. Dies gilt insbesondere für die Patienten mit einem maximalen RBD-Grad von 1. oder 2.

Hinzu kommt, dass bei der Einführung des Screening-Fragebogens durch STIASNYKOLSTER et al. (2007) Patienten mit typischen Polysomnographie-Befunden für RBD ohne entsprechende Krankengeschichte, also mit subklinischer RBD, von der Untersuchung ausgeschlossen waren. Es ist anzunehmen, dass diese Patienten weniger Fragen bejaht und damit den Schnitt gesenkt hätten. Außerdem war die RBD dort in der Patientengruppe zu $35 \%$ idiopathisch. Nur $4 \%$ der Patienten litten unter RBD und IPS. Allerdings erklärt all dies nicht, warum auch die drei DeNoPa-Kontrollpersonen mit polysomnographisch gesicherter RBD nicht durch den Fragebogen identifiziert wurden. Diese zeigten der Hypothese von EISENSEHR et al. (2001) entsprechend im Schlaflabor eine deutliche Verhaltensstörung mit RBD-Graden von minimal 2.0. Daher stellt sich die Frage, wie repräsentativ die Ergebnisse zweier exemplarischer Nächte im Schlaflabor für alle Nächte sind. Die Beobachtung RBD-typischer motorischer Aktivität in der Polysomnographie stellt eher eine Momentaufnahme dar und lässt daher keinen direkten Schluss auf Häufigkeit und intraindividuelle Schwankungen des Ausprägungsgrades in anderen Nächten zu. Bei milder RBD tritt das auffällige Verhalten im Schlaf unter Umständen seltener als ein Mal pro Monat auf (OLSON et al. 2000).

Allerdings gab es beim RBDSQ in beiden DeNoPa-Gruppen einen relativ hohen Anteil fehlender Antworten. Dies könnte unter anderem daran liegen, dass einige der Sachverhalte nur beantwortet werden können, wenn z. B. der Partner das Schlafverhalten beobachtet und 
darüber berichtet hat. Sieben Personen füllten die Frage nach lautem Sprechen, Schimpfen oder Schreien im Schlaf nicht aus. Neben dem Nicht-Wissen könnten solche Äußerungen im Schlaf auch als unangenehm und beschämend empfunden worden sein, sodass die Antwort verweigert wurde. Die gleichen Gründe könnten aber auch zur Verneinung von Fragen geführt haben.

Auf Grund der Tatsache, dass der Großteil der Studienteilnehmer mit RBD unerkannt blieb, erscheint der Fragebogen als ungeeignet für das Screening auf RBD in der Frühdiagnostik des IPS.

\subsection{Liquordiagnostik}

Die Auswertung der Liquor-Konzentration von A 342 , Tau und P-Tau ergab keinen statistisch signifikanten Unterschied zwischen De-novo-Parkinson-Patienten und Kontrollpersonen.

Dass kein Unterschied zwischen den Gruppen für die Tau- und P-Tau-Level nachweisbar war, steht im Einklang mit anderen Studien bei nicht-dementen Parkinson-Patienten (COMPTA et al. 2009, PARNETTI et al. 2008). Die einzige bisher veröffentlichte Studie zu diesen Liquorparametern bei frühen, unbehandelten Parkinson-Patienten von ALVES et al. (2010) zeigte hingegen eine signifikante Reduktion der A $\beta 42$-Level.

Bei genauerer Betrachtung der Aß42-Konzentrationen zeichnete sich auch bei den DeNoPaPatienten ein Trend hin zu niedrigeren Werten ab: Ein Drittel der Patientenergebnisse lag unter dem minimalen Wert in der Kontrollgruppe. Schon im frühen IPS-Stadium sind also bei einem Teil der Patienten substanzielle Veränderungen der A ß42-Konzentration nachweisbar. Zudem lag der Mittelwert der Patienten um 24\% niedriger als bei den Kontrollpersonen. Die Mittelwerte unterschieden sich damit sogar etwas deutlicher als in der Untersuchung von ALVES et al. (2010) (19\%) und anderen, bei denen auch nicht-demente Parkinson-Patienten mit Gesunden verglichen wurden (15-22\% bei MOLLENHAUER et al. 2006, KANEMARU et al. 2000, COMPTA et al. 2009, SJÖGREN et al. 2000, PARNETTI et al. 2008). Der Unterschied war allerdings nur bei zwei der genannten Studien signifikant (ALVES et al. 2010, PARNETTI et al. 2008). (Auf Grund möglicher Unterschiede in der Probenprozessierung ist der Mittelwertvergleich trotz der Verwendung desselben Assays (INNOTEST® $\beta$-AMYLOID $(1-42)$ der Firma Innogenetics) mit Vorsicht zu betrachten.)

Wie ALVES et al. (2010) bemerkten, sei es denkbar, dass die Heterogenität der Ergebnisse in der Patientengruppe und die Überschneidung mit den Kontrollergebnissen eine Variabilität der klinischen Manifestation (Zeitpunkt und Ausmaß) kognitiver Symptome widerspiegele und die individuellen Ergebnisse prognostische Aussagen zuließen.

Allerdings besteht wie bereits in der Einleitung erwähnt zurzeit eine grundsätzliche Kontroverse darüber, worin die neuropathologische Grundlage der Demenz bei Parkinson 
besteht. Neben Alzheimer-typischen Veränderungen und den Lewy-Körpern selbst werden auch präsynaptisch@ -Synuclein-Aggregate als Hauptursache diskutiert (SCHULZSCHAEFFER 2010). Zudem bestehen diverse komplexe Interaktionen zwischen den beteiligten Proteinen Aß42, Tau und $\alpha$-Synuclein (CLINTON et al. 2010). Die Relevanz der Liquormarker $A \beta 42$, Tau und P-Tau für die Parkinson-Demenz hängt entscheidend von den weiteren Ergebnissen prospektiver Langzeitstudien ab.

\subsection{Feasibility}

Auf Grund der Hochrechnung der Rekrutierungszahlen von September 2009 bis Juni 2010 erscheint das angestrebte Ziel, bis Dezember 2011150 Patienten und 100 Kontrollpersonen zu rekrutieren, erreichbar. Monatlich müssten dafür sechs Patienten und fünf Kontrollpersonen in die Studie eingeschlossen werden. Da im ersten Quartal von 2010 im Mittel sieben Patienten rekrutiert und im Februar neben sechs Patienten sogar acht Kontrollpersonen untersucht wurden, erscheint die Hochrechnung auch in Hinblick auf die personelle Auslastung, räumliche Kapazität und technische Ausstattung realistisch.

Dass sich bei Patienten mit dem Verdacht auf IPS bei akkurater Anamnese und Untersuchung Hinweise auf eine andere Differenzialdiagnose ergaben, reflektiert Ergebnisse von HUGHES et al. (2002). Diese zeigten, dass Neurologen mit besonderer Erfahrung auf dem Gebiet der Bewegungsstörungen allgemeinen Neurologen in Bezug auf das Eruieren und Interpretieren der entscheidenden klinischen Merkmale und damit in Blick auf die diagnostische Richtigkeit überlegen sind. Wie die Hochrechnung der Rekrutierungszahlen zeigt, bleibt die geplante Gruppengröße erreichbar, auch wenn weiterhin wenige Patienten wegen atypischer Präsentation ausgeschlossen werden müssen.

Seit Rekrutierungsbeginn kamen im Durchschnitt sechs neue Kontrollpersonen pro Monat hinzu. Eine Fortsetzung dieser Entwicklung würde bis Dezember 2011 zu einer Gruppengröße von 137 Teilnehmern führen, was die angestrebte Zahl um ein Drittel übersteigen würde.

Die vorgesehene Aufenthaltsdauer der Studienteilnehmer erwies sich als ausreichend. Die obligat geplanten Untersuchungen wurden bei den meisten Studienteilnehmern vollständig durchgeführt. Selbst die invasive Entnahme von Liquor cerebrospinalis erfolgte bei über $80 \%$ der Studienteilnehmer. Bei allen anderen Untersuchungen lag die Durchführungsrate bei mindestens $95 \%$. Allerdings gab es bei der Fragebogenerhebung einen hohen Anteil unvollständig ausgefüllter Fragebögen. 37,5\% der Probanden ließen beim Selbstbeurteilungsbogen RBDSQ Fragen unbeantwortet, $23 \%$ beim PDQ-39. In der PRIAMO-Studie zu NMS und Lebensqualität bei IPS lag die Missing-Rate für den PDQ-39 ebenfalls bei $23 \%$ (BARONE et al. 2009). Möglicherweise vergaßen die Studienteilnehmer, 
die Fragen zu beantworten oder sie verweigerten die Auskunft. Auch Nicht-Wissen ist als Ursache nicht auszuschließen, da beide Fragebögen keine Antwortmöglichkeit wie „weiß ich nicht" beinhalteten. Diese denkbaren Erklärungen wurden für den RBDSQ bereits in Kapitel 4.5.4 diskutiert. Da die Vollständigkeit ein wichtiges Kriterium für die Datenqualität und folglich für die Qualität der Ergebnisse ist, werden die Studienteilnehmer gezielt zur lückenlosen Beantwortung motiviert und der Rücklauf der Fragebögen zukünftig intensiver kontrolliert.

\subsection{Schlussfolgerung}

Durch die Pilotstudie konnte die Feasibility der DeNoPa-Kassel-Studie gezeigt werden. Den Hochrechnungen entsprechend wurden inzwischen 162 Patienten sowie 115 Kontrollpersonen eingeschlossen. Somit konnte die Rekrutierung im Januar 2012 erfolgreich abgeschlossen werden. Die Stärken der Studie liegen in dem umfassenden und standardisierten Assessment einer großen Patientenstichprobe. Die Längsschnittmethode ermöglicht eine optimale Evaluation von diagnostischen und prognostischen Biomarkern sowie von Verlauf und Formen der Erkrankung. Die Patienten sind bei Einschluss in die Studie unbehandelt, sodass keine Beeinflussung der Baseline-Ergebnisse durch Dopamin auftreten kann. Grundsätzlich ist die Sicherheit der klinischen Diagnose bei Einschluss von de novo-Patienten limitiert. Allerdings erfolgen die Erstdiagnostik sowie alle weiteren Followup-Untersuchungen durch Experten auf dem Gebiet der Bewegungsstörungen. Endgültige Sicherheit wird durch den Goldstandard, die Autopsie mit neuropathologischer Untersuchung erreicht. Durch die Rekrutierung, z. B. mittels Zeitungsanzeigen, ist eine gewisse Selektion, insbesondere der Kontrollpersonen, nicht sicher auszuschließen. Eine Verblindung der Datenerheber war in der Studienkonzeption nur für die Laboranalyse von Blut und Urin sowie für die Befundung der MRT-Untersuchungen vorgesehen. Auf Grund der vorläufig kleinen Zahl an Studienteilnehmern sind die Ergebnisse der Pilotstudie als Tendenz zu werten.

Die vergleichende Beobachtung in der Pilotstudie ergab, dass NMS des IPS bereits bei Diagnosestellung eine hohe Priorität besitzen. Bisher haben diese aber keinen Eingang in die Diagnosekriterien gefunden. Angesichts ihres gravierenden Einflusses auf die Lebensqualität ist es zudem essenziell, der Identifikation nicht-motorischer Probleme in der Patientenversorgung mehr Aufmerksamkeit zu schenken. Hilfreich kann hierbei der Einsatz des NMSQuest sein.

Die untersuchten diagnostischen Biomarkerkandidaten zeigten sich erfolgversprechend. Mit der HPS existiert ein Verfahren, das bei einer Sensitivität von fast $90 \%$ und einer Spezifität von $80 \%$ gleichzeitig schnell durchführbar, kostengünstig, nicht invasiv und weit verbreitet ist. Die Qualität der Untersuchung hängt allerdings von der Erfahrung des Untersuchers ab. 


\section{- DISKUSSION -}

Da Riechstörungen ein häufiges Frühsymptom bei IPS darstellen, ist die Verwendung eines einfachen Riechtests in der Frühdiagnostik sinnvoll. Die Polysomnographie zur RBDDiagnostik ist sehr aufwändig und nur in speziell ausgestatteten Einrichtungen durchführbar. Das Screening mittels RBDSQ ergab aber keine ausreichende Sensitivität, um die Untersuchung im Schlaflabor zu ersetzen. Die Lumbalpunktion zur Gewinnung von Liquor cerebrospinalis ist zwar eine invasive Maßnahme, weist allerdings eine niedrige Rate klinisch signifikanter unerwünschter Ereignisse auf und wird gut toleriert. Dennoch wäre es natürlich erstrebenswert, in Zukunft Marker aus einfacher zu gewinnendem Material wie Urin oder Blut zu identifizieren. Die Relevanz der Liquorproteine als prognostische Biomarker für kognitive Defizite bzw. Demenz bei Parkinson lässt sich erst retrospektiv bewerten.

Das Ziel einer sicheren, bestenfalls prämotorischen Diagnosestellung - dem Schlüsselproblem, wenn man zukünftig Neuroprotektion anstrebt - kann nur durch eine Batterie verschiedener Tests erreicht werden, die parallel oder sequenziell durchgeführt die bisherige Diagnosestrategie ergänzen. Zielsetzung der DeNoPa-Studie ist die Validierung eines solchen Algorithmus. 


\section{ZUSAMMENFASSUNG}

Vor nahezu 200 Jahren beschrieb James Parkinson erstmals die später nach ihm benannte Erkrankung, den Morbus Parkinson. Synonym wird der Begriff idiopathisches ParkinsonSyndrom (IPS) verwendet, um die neurodegenerative Erkrankung von anderen ParkinsonSyndromen mit bekannter Ursache abzugrenzen. Das IPS ist gekennzeichnet durch eine Bradykinese verbunden mit Rigor, Tremor und/ oder posturaler Instabilität. Die Diagnostik basiert bisher ausschließlich auf anamnestischen Angaben, der klinischen Untersuchung sowie dem Ansprechen auf dopaminerge Medikation. Bis heute gibt es keine anerkannten und ausreichend validierten Biomarker. Fehldiagnosen sind dementsprechend besonders in frühen Krankheitsstadien häufig. Die frühe Diagnose oder möglichenfalls die Identifikation von Risikopersonen in einem präsymptomatischen Stadium ist beim IPS besonders erstrebenswert, da die pathologischen Veränderungen zum Zeitpunkt der motorischen Erstmanifestation schon weit vorangeschritten sind. Dadurch erscheint die Wirksamkeit zukünftiger neuroprotektiver Therapien fragwürdig. Da es sich um eine Erkrankung des höheren Lebensalters handelt, wird die Prävalenz im Zuge des demographischen Wandels in Zukunft steigen.

Neben den motorischen Symptomen wurde inzwischen ein breites Spektrum nichtmotorischer Symptome des IPS beobachtet und diskutiert, darunter beispielsweise Geruchsstörungen, REM-Schlaf-Verhaltensstörung (REM-Schlaf: Schlafphase mit schnellen Augenbewegungen), orthostatische Hypotension oder kognitive Defizite. Einige dieser Symptome scheinen sich bereits in der prämotorischen Phase des IPS zu manifestieren.

Mit nicht-motorischen Symptomen bei unbehandelten Patienten mit der Verdachtsdiagnose eines IPS sowie mit potenziellen frühen Biomarkern befasst sich die hier vorliegende Pilotstudie der prospektiven Langzeit-Follow-up-Studie DeNoPa Kassel. Das Akronym DeNoPa steht hierbei für „De-novo-Parkinson“. Der Pilotstudie liegen Daten aus den Einschlussuntersuchungen der seit dem Studienbeginn im September 2009 bis Ende März 2010 eingeschlossenen Studienteilnehmer (30 Patienten und 10 Kontrollpersonen, gematcht nach Alter, Geschlecht und Bildungsstand) zu Grunde. Zielsetzung der DeNoPa-KasselStudie der Paracelsus-Elena-Klinik in Kassel ist die langfristige Verlaufsbeobachtung von insgesamt 150 Patienten. Die Kontrollgruppe soll 100 Personen ohne bekannte neurologische Erkrankung umfassen. Die Pilotstudie beinhaltet auch Untersuchungen zur Feasibility der Hauptstudie.

Die Pilotstudie zeigte, dass nicht-motorischen Symptomen des IPS bereits zu Beginn der Erkrankung ein bedeutender Stellenwert zukommt. Im Screening mittels Non-Motor Symptoms Questionnaire und Non-Motor Symptoms Scale unterschieden sich die Patienten signifikant von den Kontrollpersonen. Angesichts des frühen Krankheitsstadiums war bereits 
ein großes Spektrum potenzieller nicht-motorischer Symptome in der Patientengruppe vorhanden, auch wenn die Verteilung individuell sehr unterschiedlich war und der Ausprägungsgrad eher mild ausfiel. Zudem ließ sich mit Hilfe des Parkinson's Disease Questionnaires ein signifikanter Einfluss auf die Lebensqualität der Erkrankten nachweisen. Im Riechtest mit Hilfe der standardisierten „Sniffin“ Sticks“ hatten die Patienten insbesondere Beeinträchtigungen in der Diskriminations- und Identifikationsfähigkeit von Gerüchen. Fast $50 \%$ der Patienten waren hyposmisch und weitere $30 \%$ anosmisch. Geruchsstörungen stellen also ein häufiges Frühsymptom des IPS dar. Mit einer Sensitivität von $76 \%$ und einer Spezifität von $88 \%$ in der Unterscheidung zwischen Patienten und Kontrollpersonen, stellt der Riechtest eine sinnvolle Ergänzung der Frühdiagnostik des IPS dar.

Keinen signifikanten Unterschied zeigte hingegen der Schellongtest. Die Prävalenz der orthostatischen Hypotension lag in beiden Studiengruppen bei etwa $20 \%$ und war damit vergleichbar mit in der Allgemeinbevölkerung ermittelten Häufigkeiten. Die orthostatische Hypotension scheint also eher ein Phänomen des allgemeinen Alterns als ein typisches Frühsymptom des IPS zu sein.

Bei der Polysomnographie lag der Fokus auf dem Auftreten einer REM-SchlafVerhaltensstörung (RBD) als möglicher Frühmanifestation des IPS mit diagnostischer Relevanz. Diese war entsprechend bei $60 \%$ der frühen, unbehandelten Parkinson-Patienten prävalent. Unerwarteterweise lag der Anteil jedoch bei den Kontrollpersonen nicht signifikant niedriger. Dieses Ergebnis stellt die Aussagekraft im Rahmen der Frühdiagnostik des IPS in Frage. Das Screening mit Hilfe des RBD Screening Questionnaires zeigte zwar einen signifikanten Unterschied zwischen Patienten und Kontrollpersonen, ergab aber keine ausreichende Sensitivität, um die Untersuchung im Schlaflabor zu ersetzen.

Obwohl von verschiedenen Autoren bei ausführlicher neuropsychologischer Testung neu diagnostizierter Patienten kognitive Einschränkungen beschrieben worden sind, ergab das Screening bezüglich derartiger Defizite mit Hilfe des Mini-Mental-Status-Tests und des Uhrentests keinen signifikanten Unterschied zwischen Patienten und Kontrollpersonen. Dies deutet darauf hin, dass die Aussagekraft dieser Tests bei subtilen Veränderungen der kognitiven Fähigkeiten im frühen Stadium des IPS limitiert ist.

Die Liquorproteine $\beta$-Amyloid ${ }_{(1-42)}(\mathrm{A} \beta 42)$, Gesamt-Tau (Tau) und hyperphosphoryliertes Tau (P-Tau) sind prognostische Biomarkerkandidaten zur Identifikation von Patienten mit einem Risiko für kognitive Defizite bzw. Demenz bei Parkinson. Erwartungsgemäß unterschieden sich die Tau- und P-Tau-Level der nicht-dementen DeNoPa-Patienten nicht von denen der Kontrollpersonen. Die Aß42-Konzentrationen lagen in der Patientengruppe tendenziell niedriger, ohne hierbei aber die Signifikanzschwelle zu überschreiten. Die große Spannweite der Patienten-Ergebnisse kann potenziell eine Variabilität der klinischen Manifestation kognitiver Symptome widerspiegeln und eine individuelle prognostische Aussage zulassen. 
Ein vielversprechender diagnostischer Biomarkerkandidat ist die Echogenität der Substantia nigra, transkraniell gemessen mit Hilfe der Hirnparenchymsonographie. Diese war korrespondierend $\mathrm{zu}$ früheren Studien bei den Parkinson-Patienten beidseits signifikant höher. Eine Zuordnung zu den Studiengruppen anhand des Kriteriums Hyperechogenität versus Normoechogenität wäre für die Patienten zu $89 \%$ und für die Kontrollpersonen zu $80 \%$ korrekt gewesen. Dass die Sonographie außerdem schnell durchführbar, kostengünstig, nicht invasiv und weit verbreitet ist, spricht zusätzlich für die Nutzung in der Frühdiagnostik des IPS.

Die Untersuchung der Feasibility der DeNoPa-Kassel-Studie ergab, dass die angestrebten Rekrutierungszahlen realistisch erreichbar sind. Auch die Durchführung der geplanten Untersuchungen erwies sich als machbar. 


\section{ANHANG: ABBILDUNGEN 6-1 BIS 6-7}

Die im Anhang abgebildeten Fragebögen, Skalen und Tests wurden aus dem Case Report Form der DeNoPa-Kassel-Studie übernommen. Die Erstbeschreiber bzw. Übersetzer sind im Einzelnen in Kapitel 2 (Material und Methoden) nachgewiesen.

\section{MENTATION, BEHAVIOR, AND MOOD}

Was subscale 1 completed? Yes $\square$ No Date Performed: QUESTIONS:

1. Intellectual Impairment

$0=$ None.
$1=$ Mild. Consistent forgetfulness with partial recollection of events and no other difficultics.

$2=$ Moderate memory loss, with disorientation and moderate difficulty handling complex problems. Mild but definite

impairment of function at home with need of occasional prompting.

$3=$ Severe memory loss with disorientation for time and often to place. Severe impairment in handling problems.

$4=$ Severe memory loss with orientation preserved to person only. Unable to make judgements or solve problems.

Requires much help with personal care. Cannot be left alone at all.

2. Thought Disorder

(Due to dementia or drug intoxication)

$0=$ None.

$1=$ Vivid dreaming.

$2=$ "Benign" hallucinations with insight retained

$3=$ Occasional to frequent hallucinations or delusions; without insight; could interfere with daily activities

$4=$ Persistent hallucinations, delusions, or florrid psychosis. Not able to care for self.

\section{Depression}

$0=$ None.
$1=$ Periods of sadness or guilt greater than normal, never sustained for days or weeks.

$2=$ Sustained depression (1 week or more).

$3=$ Sustained depression with vegetative symptoms (insomnia, anorexia, weight loss, loss of interest).

$4=$ Sustained depression with vegetative symptoms and suicidal thoughts or intent.

\section{Motivation/Initiative}

$0=$ Normal.

1 - Less assertive than usual; more passive.

$2=$ Loss of initiative or disinterest in elective (nonroutine) activities.

$3=$ Loss of initiative or disinterest in day to day (routine) activities.

4 = Withdrawn, complete loss of motivation.

II. ACTIVITIES OF DAILY LIVING ]

Was subscale 2 completed? $\quad \square$ Yes $\quad \square$ No Date Performec

QUESTIONS:

Date Performed: $\frac{}{\mathrm{M}} \frac{\mathrm{l}}{\mathrm{M}} \frac{1}{\mathrm{D}} \frac{\mathrm{l}}{\mathrm{D}} \frac{1}{\mathrm{Y}} \frac{1}{\mathrm{Y}} \frac{}{\mathrm{Y}} \frac{}{\mathrm{Y}}$

5. Speech

$0=$ Normal.

1 = Mildly affected. No difficulty being understood.

$2=$ Moderately affected. Sometimes asked to repeat statements.

$3=$ Severely affected. Frequently asked to repeat statements.

$4=$ Unintelligible most of the time.

\section{Salivation}

$0=$ Normal.

$0=$ Normal.
$1=$ Slight but definite excess of saliva in mouth; may have nighttime drooling.

$2=$ Moderately excessive saliva; may have minimal drooling.

$3=$ Marked excess of saliva with some drooling.

$4=$ Marked drooling, requires constant tissue or handkerchief.

\section{Swallowing}

$0=$ Normal.

$1=$ Rare choking.

$2=$ Occasional choking

$3=$ Requires sott food

whe or gastrotomy feeding

8. Handwriting

$0=$ Normal.

$1=$ Slightly slow or small.

$2=$ Moderately slow or small; all words are legible.

3 - Severely affected; not all words are legible.

$4=$ The majority of words are not legible.

9. Cutting food and handling utensils

$0=$ Normal.

$1=$ Somewhat slow and clumsy, but no help needed.

$2=$ Can cut most foods, although clumsy and slow; some help needed.

$3=$ Food must be cut by someone, but can still feed slowly.

$4=$ Needs to be fed.

\section{Dressing}

$0=$ Normal.

1 = Somewhat slow, but no help needed.

$2=$ Occasional assistance with buttoning, getting arms in sleveres.

3 = Considerable help req̣uired, but can do some things alone,

$4=$ Helpless.

11. Hygiene

$0=$ Normal.

$1=$ Somewhat slow, but no help needed.

$2=$ Needs help to shower or bathe; or very slow in hygienic care.

$3=$ Requires assistance for washing, brushing teeth, combing hair, going to barhroom

4 - Foley catheter or other mechanical aids. 
12. Turning in bed and adjusting bed clothes $0=$ Normal.

$0=$ Normal.
$1=$ Somewhat slow and clumsy, but no help needed.

$1=$ Somewhat slow and clumsy, but no help needed.
2 = Can turn alone or adjust sheets, but with great difficulty.

$2=$ Can turn alone or adjust sheets, but with grcat dist
$3=$ Can initiate, but not turn or adjust shects alone.

$4=$ Helpless.

13. Falling (unrelated to freezing)

$0=$ None

$1=$ Rare falling.

$2=$ Occasionally falls, less than once per day.

$3=$ Falls an average of once daily

$4=$ Falls more than once daily.

\section{Freezing when walking}

$0=$ None.

$1=$ Rare freezing when walking; may have starthesitation.

$2=$ Occasional freezing when walking .

$3=$ Frequent freezing. Occasionally falls from freezing.

$4=$ Frequent falls from freezing.

\section{Walking}

$0=$ Normal.

$1=$ Mild difficulty. May not swing arms or may tend to drag leg.

$2=$ Moderate difficulty, but requires little or no assistance.

$3=$ Severe disturbance of walking, requiring assistance.

$4=$ Cannot walk at all, even with assistance.

\section{Tremor}

(Symptomatic complaint of tremor in any part of body.)

$0=$ Absent.

$1=$ Slight and infrequently present.

$2=$ Moderate; bothersome to patient.

$3=$ Severe; interferes with many activities.

$4=$ Marked; interferes with most activitics.

17. Sensorv complaints related to parkinsonism

$0=\mathrm{None}$

$1=$ Occasionally' has numbness, tingling, or mild acning.

$1=$ Occasionally has numbness, tingling, or mild acning,
$2=$ Frequently has numbness, tingling, or aching; not distressing.

$2=$ Frequently has numbness, tin
$3=$ Frequent painful sensations.

$4=$ Excruciating pain.

\section{MOTOR EXAMINATION}

Was subscale 3 completed?

$\square$ Yes

Date Performed:

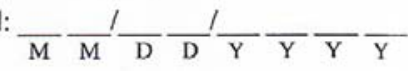

QUESTIONS:

SCORE:

18. Speech

$0=$ Normal.

$1=$ Slight loss of expression, diction and/or volume.

2 = Monotone, slurred but understandable; moderately impaired.

$3=$ Marked impairment, difficult to understand.

$4=$ Unintelligible.

19. Facial Expression

$0=$ Normal.

1 = Minimal hypomimia, could be normal "Poker Face".

$2=$ Slight but definitely abnormal diminution of facial expression

$3=$ Moderate hypomimia; lips parted some of the time.

$4=$ Masked or fixed facies with severe or complete loss of facial expression; lips parted 1/4 inch or more.

20. Tremor at rest

(head, upper and lower extremities)

$0=$ Absent.

$1=$ Slight and infrequently present.

$2=$ Mild in amplitude and persistent. Or moderate in amplitude, but only intermittently present.

$3=$ Moderate in amplitude and present most of the time.

$4=$ Marked in amplitude and present most of the time.

20a. Face, lips+chin

20a.

20b. Right Hand

20b.

20c. Left Hand

20c.

20d. Right Foot

20d.

20e. Left Foot

20 e.

Fortsetzung Abbildung 6-1: Unified Parkinson Disease Rating Scale (UPDRS), Fraqen 12-20/42 
21. Action or Postural Tremor of hands $0=$ Absent.

$1=$ Slight; present with action

$2=$ Moderate in amplitude, present with action

$3=$ Moderate in amplitude with posture holding as well as action.

$4=$ Marked in amplitude; interferes with feeding

21a. Right Hand

2lb. Left Hand 2la.

21b.

\section{Rigidity}

(Judged on passive movement of major joints with patient relaxed in sitting position. Cogwheeling to be ignored.)

$0=$ Absent.

$1=$ Slight or detectable only when activated by mirror or other movements.

$2=$ Mild to moderate.

$3=$ Marked, but full range of motion easily achieved.

$4=$ Severe, range of motion achieved with difficulty.

22a. Neck

22b. RUE

22c. LUE

22d. RLE

22e. LLE

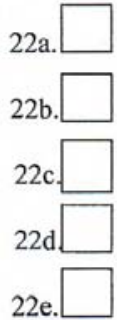

23. Finger Taps

(Patient taps thumb with index finger in rapid succession.)

$0=$ Normal.

$1=$ Mild slowing and/or reduction in amplitude.

2 = Moderately impaired. Definite and early fatiguing. May have occasional arrests in movement.

$3=$ Severely impaired. Frequent hesitation in initiating movements or arrests in ongoing movement.

$4=$ Can barely perform the task.

23a. Right Hand

23a.

$23 \mathrm{~b}$.

\section{Hand Movements}

(Patient opens and closes hands in rapid succesion.)

$0=$ Normal.

$1=$ Mild slowing and/or reduction in amplitude.

$2=$ Moderately impaired. Definite and early fatiguing. May have occasional arrests in movement.

$3=$ Severely impaired. Frequent hesitation in initiating movements or arrests in ongoing movement.

$4=$ Can barely perform the task.

24a. Right Hand

24b. Left Hand

$24 \mathrm{~b}$

25. Rapid Alternating Movements of Hands

(Pronation-supination movements of hands, vertically and horizontally,

with as large an amplitude as possible, both hands simultaneously.)

$0=$ Normal.

$1=$ Mild slowing and/or reduction in amplitude.

$2=$ Moderately impaired. Definite and early fatiguing. May have occasional arrests in movement.

$3=$ Severely impaired. Frequent hesitation in initiating movements or arrests in ongoing movement.

$4=$ Can barely perform the task. 
26. Leg Agility

(Patient taps heel on the ground in rapid succession picking up entire leg. Amplitude should be at least

3 inches.)

$0=$ Normal.

$1=$ Mild slowing and/or reduction in amplitude.

$2=$ Moderately impaired. Definite and early fatiguing. May have occasional arrests in movement.

$3=$ Severely impaired. Frequent hesitation in initiating movements or arrests in ongoing movement.

$4=$ Can barely perform the task.

26a. Right Leg

26a. $\square$

26b. Left Leg

26b.

27. Arising from Chair

(Patient attempts to rise from a straightbacked chair, with arms folded across chest.)

$0=$ Normal.

$1=$ Slow; or may need more than one attempt.

$2=$ Pushes self up from arms of seat.

$3=$ Tends to fall back and may have to try more than one time, but can get up without help.

$4=$ Unable to arise without help.

\section{Posture}

$0=$ Normal erect.

1 = Not quite erect, slightly stooped posture; could be normal for older person.

$2=$ Moderately stooped posture, definitely abnormal; can be slightly leaning to one side.

$3=$ Severely stooped posture with kyphosis; can be moderately leaning to one side.

$4=$ Marked flexion with extreme abnormality of posture.

\section{Gait}

$0=$ Normal.

I = Walks slowly, may shuffle with short steps, but no festination (hastening steps) or propulsion.

2 = Walks with difficulty, but requires little or no assistance; may have some festination, short steps, or propulsion.

$3=$ Severe disturbance of gait, requiring assistance.

$4=$ Cannot walk at all, even with assistance.

\section{Postural Stability}

(Response to sudden, strong posterior displacement produced by pull on shoulders while patient

erect with eyes open and feet slightly apart. Patient is prepared.)

$0=$ Normal.

$1=$ Retropulsion, but recovers unaided.

2 = Absence of postural response; would fall if not caught by examiner.

$3=$ Very unstable, tends to lose balance spontaneously.

$4=$ Unable to stand without assistance.

31. Body Bradykinesia and Hypokinesia

(Combining slowness, hesitancy, decreased armswing, small amplitude, and

poverty of movement in general.)

$0=$ None.

$1=$ Minimal slowness, giving movement a deliberate character; could be normal for some persons. Possibly reduced

amplitude.

$2=$ Mild degree of slowness and poverty of movement which is definitely abnormal. Alternatively, some reduced amplitude.

3 = Moderate slowness, poverty or small amplitude of movement.

4 = Marked slowness, poverty or small amplitude of movement.

Fortsetzung Abbildung 6-1: Unified Parkinson Disease Rating Scale (UPDRS), Fragen 26-31/42 
IV. COMPLICATIONS OF THERAPY (In the past week) ]

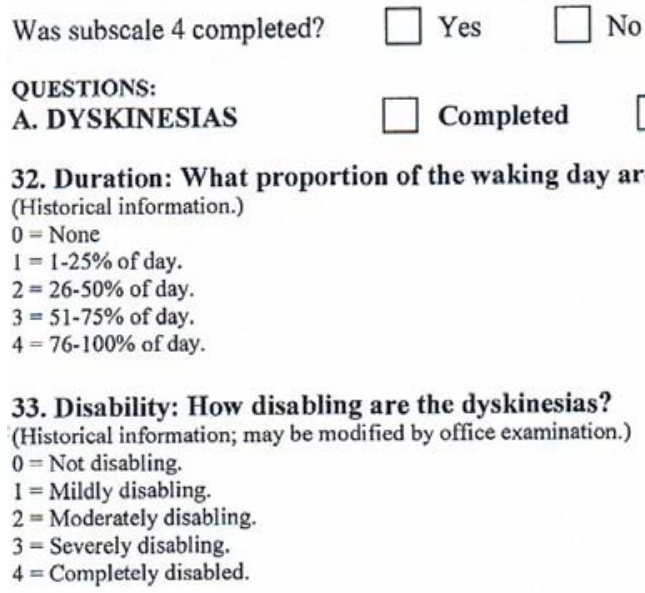

33. Disability: How disabling are the dyskinesias?

(Historical information; may be modified by office examination.)

$0=$ Not disabling.

$1=$ Mildly disabling.

$2=$ Moderately disabling.

$3=$ Severely disabling.

$4=$ Completely disabled.

34. Painful Dyskinesias: How painful are the dyskinesias?

$0=$ No painful dyskinesias.

$1=$ Slight.

$2=$ Moderate

$3=$ Severe.

$4=$ Marked.

35. Presence of Early Morning Dystonia

(Historical information.)

$0=$ No

$1=$ Yes

B. CLINICAL FLUCTUATIONS

36. Are "off" periods predictable?

$0=$ No

$1=$ Yes

37. Are "off" periods unpredictable?

$0=$ No

$1=$ Yes

38. Do "off" periods come on suddenly, within a few seconds?

$0=$ No

$1=$ Yes

39. What proportion of the waking day is the patient "off" on average?

$0=$ None

$1=1-25 \%$ of day.

$2=26-50 \%$ of day.

$3=51-75 \%$ of day

$4=76-100 \%$ of day.

\section{OTHER COMPLICATIONS}

Completed

Not Completed

40. Does the patient have anorexia, nausea, or vomiting? $0=$ No

$1=$ Yes

41. Any sleep disturbances, such as insomnia or hypersomnolence?

$0=$ No

$1=$ Yes

42. Does the patient have symptomatic orthostasis?

( Record the patient's blood pressure, height and weight on the scoring form)

$0=$ No

$1=$ Yes

Fortsetzung Abbildung 6-1: Unified Parkinson Disease Rating Scale (UPDRS), Fragen 32-42/42 
- ANHANG: ABBILDUNGEN 6-1 BIS 6-7 -

\section{Der Uhren-Test (modifiziert nach Shulman 1993)}

Anweisungen zur Durchführung:

1. Geben Sie dem Patienten ein Blatt Papier mit einem vorgezeichneten Kreis. Zeigen Sie ihm, wo oben und unten ist.

2. Geben Sie dem Patienten folgende Anweisung: „Dies soll eine Uhr sein. Ich möchte Sie bitten, in diese Uhr die fehlenden Ziffern zu schreiben. Zeichnen Sie danach die Uhrzeit ,10 nach 11' ein."

3. Machen Sie sich Notizen zur Ausführung der gestellten Aufgabe (Reihenfolge, Korrekturen etc.).

4. Bewerten Sie die angefertigte Zeichnung gemäß der unten stehenden Kriterien. Notieren Sie den Score zusammen mit Datum und Namen des Patienten auf dem Zeichenblatt.

5. Der validierte Cut-Off zur Unterscheidung zwischen Normalbefund einerseits und kognitiver Beeinträchtigung im Sinne einer evtl. vorliegenden Demenz andererseits liegt zwischen 2 und 3. Anders ausgedrückt: Ein Score von $\geq 3$ Punkten ist als pathologisch anzusehen.

Bewertung ( 1 = ohne Fehler, 6 = keine Uhr erkennbar)

\begin{tabular}{|c|c|c|}
\hline Score & Beschreibung & Beispiele \\
\hline 1 & $\begin{array}{l}\text { „Perfekt" } \\
\text { - Ziffern 1-12 richtig eingezeichnet } \\
\text { - zwei Zeiger, die die richtige Uhrzeit (11:10 Uhr) anzeigen }\end{array}$ & \\
\hline 2 & $\begin{array}{l}\text { Leichte visuell-räumliche Fehler } \\
\text { - Abstände zwischen den Ziffern nicht gleichmäßig } \\
\text { - Ziffern außerhalb des Kreises } \\
\text { - Blatt wird gedreht, so dass die Ziffern auf dem Kopf stehen } \\
\text { - Pat. verwendet Linien („Speichen“) zur Orientierung }\end{array}$ & \\
\hline 3 & $\begin{array}{l}\text { Fehlerhafte Uhrzeit bei erhaltener visuell-räumlicher } \\
\text { Darstellung der Uhr } \\
\text { - nur ein Zeiger } \\
\text { - „10 nach } 11^{\prime \prime} \text { (o. a..) als Text hingeschrieben } \\
\text { - keine Uhrzeit eingezeichnet }\end{array}$ & \\
\hline 4 & $\begin{array}{l}\text { Mittelgradige visuell-räumliche Desorganisation, so dass } \\
\text { ein korrektes Einzeichnen der Uhrzeit unmöglich wird } \\
\text { - unregelmäßige Zwischenräume } \\
\text { - Ziffern vergessen } \\
\text { - Perserveration: wiederholt den Kreis, Ziffern jenseits der } 12 \\
\text { - Rechts-links-Umkehr (Ziffern gegen den Uhrzeigersinn) } \\
\text { - Dysgraphie - keine lesbare Darstellung der Ziffern }\end{array}$ & \\
\hline 5 & $\begin{array}{l}\text { Schwergradige visuell-räumliche Desorganisation } \\
\text { - wie unter (4) beschrieben, aber stärker ausgeprägt }\end{array}$ & \\
\hline 6 & $\begin{array}{l}\text { Keinerlei Darstellung einer Uhr } \\
\text { (cave: Ausschluss Depression/Delir!) } \\
\text { - kein wie auch immer gearteter Versuch, eine Uhr zu zeichnen } \\
\text { - keine entfernte Ähnlichkeit mit einer Uhr } \\
\text { - Patient schreibt Worte oder Name }\end{array}$ & \\
\hline
\end{tabular}

Literatur:

. Shulman KI, Shedletsky R, Silver IL. The challenge of time: Clock-drawing and cognitive function in the elderly. Int J Geriatr Psychiatry 1986, 1:135-140. 2. Shulman KI, Gold DP et al. Clock-drawing and dementia in the community: a longitudinal study. Int $J$ Geriatr Psychiatry 1993, 8:487-496.

3. Brodaty H, Moore CM. The Clock Drawing Test for dementia of the Alzheimer's type: a comparison of three scoring methods in a memory disorders clinic. Int J Geriatr Psychiatry 1997, 12:619-627. 
- ANHANG: ABBILDUNGEN 6-1 BIS 6-7 -

\section{Orientierung}

In welchem Jahr leben wir?

Welche Jahreszeit ist jetzt?

Welches Datum haben wir heute?

Welchen Tag haben wir heute?

Welchen Monat haben wir?

In welchem Bundesland sind wir hier?

In welchem Land?

In welcher Ortschaft?

Wo sind wir (in welcher Praxis, Altenheim)?

Auf welchem Stockwerk?

\section{Merkfähigkeit}

Fragen Sie den Patienten, ob Sie sein Gedächtnis prüfen dürfen. Nennen Sie dann drei verschiedenartige Dinge klar und langsam (ca. 1 pro sec.): "Zitrone, Schlüssel, Ballu. Nachdem Sie alle drei Wörter ausgesprochen haben, soll der Patient sie wiederholen. Die erste Wiederholung bestimmt die Wertung $(0-3$, vergeben Sie für jedes wiederholte Wort 1 Punkt), doch wiederholen Sie den Versuch, bis der Patient alle drei Wörter nachsprechen kann; maximal gibt es 5 Versuche. Wenn ein Patient nicht alle drei Wörter lernt, kann das Erinnern nicht sinnvoll geprüft werden.

Punkte 0 - 3

\section{Aufmerksamkeit und Rechnen}

Bitten Sie den Patienten, bei 100 beginnend in 7erSchritten rückwärts zu zählen. Halten Sie nach 5 Subtraktionen $(93,86,79,72,65)$ an und zählen Sie die in der richtigen Reihenfolge gegebenen Antworten. Bitten Sie daraufhin, das Wort "Preis" rückwärts zu buchstabieren. Die Wertung entspricht der Anzahl Buchstaben in der richtigen Reihenfolge (z.B. SIERP = 5, SIREP = 3). Die höhere der beiden Wertungen wird gezählt.

Punkte 0 - 5

\section{Erinnern}

Fragen Sie den Patienten, ob er die Wörter noch weiß, die er vorhin auswendig lernen sollte. Geben Sie einen Punkt für jedes richtige Wort.

Punkte 0 - 3

\section{Benennen}

Zeigen Sie dem Patienten eine Armbanduhr und fragen Sie inn, was das ist. Wiederholen Sie die Aufgabe mit einem Bleistift. Geben Sie einen Punkt für jeden erfüllten Aufgabenteil.

Punkte 0 - 2

\section{Wiederholen}

Bitten Sie den Patienten, den Ausdruck "Kein Wenn und Aber" nachzusprechen. Nur ein Versuch ist erlaubt.

Punkte 0 - 1

\section{Dreiteiliger Befehl}

Lassen Sie den Patienten den folgenden Befehl ausführen: „Nehmen Sie ein Blatt in die Hand, falten Sie es in der Mitte und legen Sie es auf den Boden!". Geben Sie einen Punkt für jeden richtig ausgeführten Befehl.

Punkte 0 - 3

\section{Reagieren}

Schreiben Sie auf ein weißes Blatt in großen Buchstaben "Schließen Sie die Augen". Der Patient soll den Text lesen und ausführen. Geben Sie einen Punkt, wenn der Patient die Augen schließt.

(siehe Rückseite)

Punkte 0 - 1

\section{Schreiben}

Geben Sie dem Patienten ein weißes Blatt, auf das er für Sie einen Satz schreiben soll. Diktieren Sie den Satz nicht, er soll spontan geschrieben werden. Der Satz muss ein Subjekt und ein Verb enthalten und einen Sinn ergeben. Korrekte Grammatik und Interpunktion werden nicht verlangt.

(siehe Rückseite)

Punkte 0 - 1

\section{Abzeichnen}

Zeichnen Sie auf ein weißes Blatt zwei sich überschneidende Fünfecke und bitten Sie den Patienten, die Figur genau abzuzeichnen. Alle 10 Ecken müssen vorhanden sein und 2 müssen sich überschneiden, um als 1 Punkt zu zählen. Zittern und Verdrehung der Figur sind nicht wesentlich.

(siehe Rückseite) Punkte 0 - 1

Summe der Punkte

\section{Abbildung 6-3: Mini-Mental-Status-Test (MMST)}




\section{Fragebogen zu nicht-motorischen Symptomen beim Morbus Parkinson (PD NMS Questionnaire)}

Name:

Alter:

Datum:

Klinik/Zentrum:

Mann $\square \quad$ Frau

Nicht die Bewegung betreffende Probleme bei der Parkinson-Erkrankung

Die Bewegungsstörungen bei der Parkinson-Erkrankung sind gut bekannt. Es können aber manchmal auch andere Probleme auftreten, als Teil der Erkrankung oder deren Behandlung. Es ist wichtig, dass der Arzt über diese Probleme Bescheid weiß, v.a. wenn sie von Ihnen als störend empfunden werden.

Eine Reihe von Problemen ist unten angeführt. Bitte kreuzen Sie das Feld "Ja“ an, wenn Sie das beschriebene Symptom während des letzten Monats erlebt haben. Der Arzt oder die Krankenschwester kann Ihnen Fragen stellen, um Ihnen bei der Entscheidung zu helfen. Wenn bei Innen das jeweilige Problem im Laufe des letzten Monats nicht aufgetreten ist, kreuzen Sie bitte das Feld "Nein" an. Sie sollten auch dann "Nein" antworten, wenn Sie die Symptome in der Vergangenheit, aber nicht während des letzten Monats hatten.

Ist bei Ihnen innerhalb des letzten Monats Folgendes aufgetreten?

JA NEIN

1. Herauslaufen von Speichel tagsüber.

…

2. Verlust oder Veränderung in Ihrer Fähigkeit zu schmecken oder zu riechen

3. Schwierigkeit beim Schlucken von Nahrung oder Getränken oder Probleme mit Verschlucken...

4. Erbrechen oder Gefühl von Übelkeit

5. Verstopfung (weniger als 3 Stuhlentleerungen pro Woche) oder Notwendigkeit beim Stuhlgang stark zu pressen.

6. Stuhlinkontinenz

7. Gefühl der unvollständigen Darmentleerung nach dem Toilettengang

8. Plötzlicher starker Harndrang, so dass Sie sich beeilen müssen, zur Toilette zu gehen

9. Regelmäßiges năchtliches Aufstehen zum Wasserlassen

10. Unerklärliche Schmerzen (nicht als Folge bekannter Erkrankungen wie z.B. Arthritis)

口..

11. Unerklärliche Gewichtsveränderungen (nicht als Folge geänderter Ernährung).

12. Probleme sich an Dinge zu erinnern, die kürzlich passiert sind, oder vergessen, Dinge zu erledigen.

13. Interesseverlust an dem was um Sie herum geschieht, oder an Aktivităten

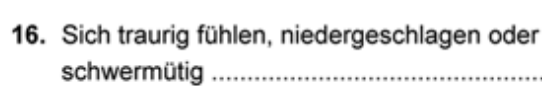

JA NEIN

17. Gefühl der Angst, Furcht oder Panik.

18. Reduziertes oder gesteigertes Interesse an Sex.

19. Gefühl von Schwierigkeiten beim Versuch Geschlechtsverkehr zu praktizieren.

20. Gefühl von Blutleere im Kopf, Schwindel oder Schwăche beim Aufstehen aus dem Sitzen oder Liegen

21. Stürze

22. Schwierigkeiten, während Aktivitäten wie Arbeit, Autofahren oder Essen wach zu bleiben ..

23. Schwierigkeiten abends einzuschlafen oder nachts durchzuschlafen.

24. Intensive lebhafte Träume oder Trăume, die Angst machen

25. Sprechen oder Bewegungen während des Schlafs, so als ob Sie einen Traum „ausleben“

26. Unangenehme Empfindungen in Ihren Beinen nachts oder beim Ausruhen und das Gefühl, sich bewegen zu müssen

Sehen oder Hören von Dingen, von denen Sie wissen oder Ihnen gesagt wird, dass sie nicht da sind.

27. Geschwollene Beine

28. Übermäßiges Schwitzen

29. Doppelbilder

30. Glauben, dass Ihnen Dinge passieren, von denen andere sagen, dass sie nicht wahr sind.

15. Schwierigkeiten, sich zu konzentrieren oder aufmerksam zu bleiben

Alle Informationen, die Sie in diesem Formular angeben, werden vertraulich behandelt und nur zu dem Zweck verwendet, für die sie erhoben wurden. Die angegebenen Informationen werden zur Verlaufskontrolle benutzt. Ihre persönlichen Daten werden in Übereinstimmung mit dem Datenschutzgesetz verwendet und aufbewahrt.

Entwickelt und validiert von der International PD Non-Motor Group, deutsche Version von Jost W, Odin P, Storch A. @Chaudhuri KR, Jost W, Odin P, Storch A, 2009. For request: Alexander.Storch@uniklinikum-dresden.de

\section{Abbildung 6-4: Non-Motor Symptoms Questionnaire (NMSQuest)}




\section{Skala zur Erfassung nicht-motorischer Symptome bei der Parkinson-Erkrankung (Non-Motor Symptoms assessment scale for Parkinson's disease)}

Name:

Alter:

Datum:

Klinik/Zentrum:

Mann

Frau

Symptome, die während des letzten Monats auftraten werden erfasst. Jedes Symptom wird bewertet wie folgt:

Ausprägung: 0 = keine, 1 = leicht: Symptome vorhanden, aber verursachen wenig Belastungen oder Beeinträchtigung des Patienten. 2 = mäßig: mäßige Belastung oder Beeinträchtigung für den Patienten. 3 = schwer: erhebliche Belastung oder Beeinträchtigung des Patienten.

Häufigkeit: 1 = selten (<1/Woche), 2 = gelegentlich (1/Woche), 3 = häufig (mehrere Mal pro Woche), $4=$ sehr häufig (täglich oder ständig)

Die einzelnen Bereiche werden unterschiedlich gewichtet. Ja / Nein Antworten werden nicht in die abschließende Häufigkeit $x$ Schwere-Berechnung eingeschlossen

(Der bei den Fragen in Klammern gesetzte Text wurde als Erklärungshilfe eingefügt).

Bereich 1: Kardiovaskulär, einschl. Stürze

1. Leidet der Patient unter Benommenheit, Schwindel oder Schwäche beim Aufstehen aus dem Sitzen oder aus liegender Position?

2. Stürzt der Patient aufgrund von Ohnmacht oder plötzlichem Bewusstseinsverlust?

Summenwert:

\section{Bereich 2: Schlaf / Müdigkeit}

3. Kommt es bei dem Patienten zu unwillkürlichem Wegdämmern oder Einschlafen während alltäglicher Aktivitäten (zum Beispiel während Unterhaltungen, bei den Mahlzeiten oder beim Fernsehen oder Lesen)?

4. schränken Müdigkeit oder Energiemangel (nicht Verlangsamung) die alltäglichen Aktivităten des Patienten ein?

5. Hat der Patient Probleme ein- oder durchzuschlafen?

6. Fühlt der Patient wenn er / sie ruhig sitzt oder liegt den Drang die Beine zu bewegen oder Unruhe in den Beinen und bessern sich diese Beschwerden bei Bewegungen?

Summenwert:

\section{Bereich 3: Stimmung / Kognition}

7. Hat der Patient das Interesse an ihrer / seiner Umgebung verloren?

8. Hat der Patient das Interesse an Aktivitäten verloren oder verminderte Motivation neue Aktivitäten zu beginnen?

9. Fühlt sich der Patient ohne erkennbaren Grund nervős, besorgt oder ăngstlich?

10. Erscheint der Patient traurig oder deprimiert oder hat er / sie derartige Gefühle angegeben?

11. Hat der Patient eine verflachte Stimmungslage ohne die normalen „Hochs" und "Tiefs"?

12. Hat der Patient Probleme, bei seinen üblichen Aktivitäten Freude zu empfinden oder berichtet er, dass inm Freude fehlt?

Summenwert:

Bereich 4: Wahrnehmungsprobleme / Halluzinationen

13. Gibt der Patient an dass er / sie Dinge sieht die nicht vorhanden sind?

14. Hat der Patient Wahrnehmungen, von denen Sie wissen, dass sie nicht der Realität entsprechen (z. B. die Vorstellung geschädigt, beraubt oder betrogen zu werden)?

15. Leidet der Patient unter Doppelbildern (2 separate reale Dinge und nicht verschwommenes Sehen)?

Summenwert: 
16. Hat der Patient Probleme, die Konzentration während Aktivităten aufrecht zu erhalten (z. B. beim Sprechen oder bei Unterhaltungen)?

17. Vergisst der Patient Dinge, die ihr / ihm eine kurze Zeit vorher erzählt wurden oder Ereignisse, die sich in den letzten Tagen ereignet haben?

18. Vergisst der Patient Dinge zu erledigen (z. B. Tabletten einnehmen oder Haushaltsgeräte auszustellen)?

Summenwert:

Bereich 6: Gastrointestinaler Trakt

19. Hat der Patient tagsüber Speichelfluss?

20. Hat der Patient Probleme beim Schlucken?

21. Leidet der Patient an Verstopfung (Stuhlentleerung weniger als $3 \times$ pro Woche)

Summenwert:

Bereich 7: Miktion

22. Hat der Patient Probleme, den Urin zu halten (Harndrang)?

23. Muss der Patient innerhalb von 2 Std. nach dem letzten Urinieren Wasserlassen (Pollakisurie)?

24. Muss der Patient regelmäßig nachts aufstehen, um Wasser zu lassen (Nykturie)?

Summenwert:

Bereich 8: Sexualfunktionen

25. Hat der Patient ein verăndertes Interesse an Sexualităt (deutlich gesteigert oder vermindert, bitte unterstreichen)?

26. Hat der Patient Probleme beim Geschlechtsverkehr?

Summenwert:

\section{Bereich 9: Verschiedenes}

27. Leidet der Patient an Schmerzen, die nicht durch andere Ursachen erklärt werden (stehen diese in Beziehung zu einer Medikamenteneinnahme und werden sie durch Antiparkinson-Medikamente gebessert)?

28. Gibt der Patient Veränderungen bei der Fähigkeit zu Riechen oder zu Schmecken an?

29. Gibt der Patient aktuell eine Gewichtsveränderung an (nicht durch Diät bedingt)?

30. Leidet der Patient an exzessivem Schwitzen (nicht durch warmes Wetter bedingt)

Summenwert:
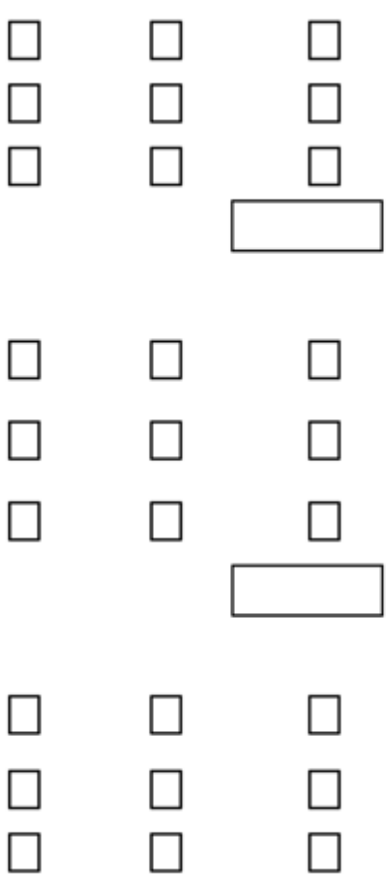


\begin{tabular}{|c|c|c|c|c|c|c|}
\hline & $\begin{array}{l}\text { Wie oft haben Sie im letzten Monat wegen ihrer } \\
\text { Parkinson Erkrankung... }\end{array}$ & 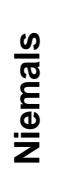 & 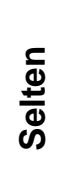 & 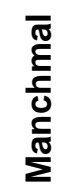 & 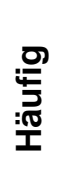 & 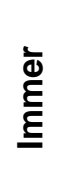 \\
\hline 1. & $\begin{array}{l}\text {...Schwierigkeiten gehabt, Freizeitaktivitäten, die sie gerne } \\
\text { machen würden auszuüben? }\end{array}$ & & & & & \\
\hline 2. & $\begin{array}{l}\text {...Schwierigkeiten gehabt, ihren Haushalt zu } \\
\text { versorgen? }\end{array}$ & & & & & \\
\hline 3. & ...Schwierigkeiten gehabt, Einkaufstaschen zu tragen? & & & & & \\
\hline 4. & ...Probleme gehabt, ungefähr 1 km zu gehen? & & & & & \\
\hline 5. & ...Probleme gehabt, ungefähr 100 m zu gehen? & & & & & \\
\hline 6. & $\begin{array}{l}\text {...Probleme gehabt, sich im Haus so zu bewegen, wie Sie } \\
\text { wollten? }\end{array}$ & & & & & \\
\hline 7. & $\begin{array}{l}\text {...Probleme gehabt, sich in der Öffentlichkeit zu } \\
\text { bewegen? }\end{array}$ & & & & & \\
\hline 8. & $\begin{array}{l}\text {...eine Begleitperson gebraucht, um sich außer Haus zu } \\
\text { bewegen? }\end{array}$ & & & & & \\
\hline 9. & $\begin{array}{l}\text {...Angst oder Sorgen gehabt, dass Sie in der } \\
\text { Öffentlichkeit hinfallen? }\end{array}$ & & & & & \\
\hline 10. & ...das Gefühl gehabt, mehr an das Haus gebunden zu sein? & & & & & \\
\hline 11. & ...Schwierigkeiten gehabt, sich selbst zu waschen? & & & & & \\
\hline 12. & ...Schwierigkeiten gehabt, sich selbst anzuziehen? & & & & & \\
\hline 13. & $\begin{array}{l}\text {...Probleme gehabt, Knöpfe zu schließen oder } \\
\text { Schnürsenkel zu binden? }\end{array}$ & & & & & \\
\hline 14. & ...Probleme gehabt, deutlich zu schreiben? & & & & & \\
\hline 15. & ...Schwierigkeiten gehabt, ihr Essen klein zu schneiden? & & & & & \\
\hline 16. & $\begin{array}{l}\text {...Schwierigkeiten gehabt, ein Getränk zu halten, ohne es zu } \\
\text { verschütten? }\end{array}$ & & & & & \\
\hline 17. & ...sich niedergeschlagen oder deprimiert gefühlt? & & & & & \\
\hline 18. & ...sich isoliert oder einsam gefühlt? & & & & & \\
\hline 19. & ...sich verärgert oder verbittert gefühlt? & & & & & \\
\hline 20. & ...sich den Tränen nahe gefühlt? & & & & & \\
\hline 21. & ...sich ängstlich gefühlt? & & & & & \\
\hline 22. & ...sich Sorgen über die Zukunft gemacht? & & & & & \\
\hline 23. & $\begin{array}{l}\text {...das Gefühl gehabt, ihre Parkinson Erkrankung vor anderen } \\
\text { verheimlichen zu müssen? }\end{array}$ & & & & & \\
\hline 24. & $\begin{array}{l}\text {...Situationen vermieden, die mit dem Essen oder } \\
\text { Trinken in der Öffentlichkeit verbunden waren? }\end{array}$ & & & & & \\
\hline 25. & $\begin{array}{l}\text {...sich in der Öffentlichkeit wegen ihrer Erkrankung } \\
\text { geschämt? }\end{array}$ & & & & & \\
\hline
\end{tabular}

Abbildung 6-6: Parkinson's Disease Questionnaire (PDQ-39), Fragen 1-25/39 
- ANHANG: ABBILDUNGEN 6-1 BIS 6-7 -

\begin{tabular}{|c|c|c|c|c|c|c|}
\hline & $\begin{array}{l}\text { Wie oft haben Sie im letzten Monat wegen ihrer } \\
\text { Parkinson Erkrankung... }\end{array}$ & 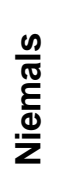 & $\begin{array}{l}\frac{5}{\Phi} \\
\frac{ \pm}{\Phi !}\end{array}$ & 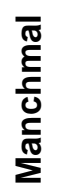 & 莺 & 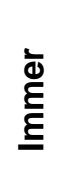 \\
\hline 26. & $\begin{array}{l}\text {...sich Sorgen über die Reaktionen anderer ihnen gegenüber } \\
\text { gemacht? }\end{array}$ & & & & & \\
\hline 27. & $\begin{array}{l}\text {...Probleme im Verhältnis mit Ihnen nahe stehenden } \\
\text { Menschen gehabt? }\end{array}$ & & & & & \\
\hline 28. & $\begin{array}{l}\text {...nicht die Unterstützung erhalten, die Sie von Ihrem (Ehe-) } \\
\text { Partner benötigt hätten? }\end{array}$ & & & & & \\
\hline 29. & $\begin{array}{l}\text {...nicht die Unterstützung erhalten, die Sie von ihren } \\
\text { Verwandten oder engen Freunden benötigt hätten? }\end{array}$ & & & & & \\
\hline 30. & $\begin{array}{l}\text {...das Problem gehabt, tagsüber unerwartet } \\
\text { einzuschlafen? }\end{array}$ & & & & & \\
\hline 31. & $\begin{array}{l}\text {...Probleme gehabt, sich zu konzentrieren (z.B. beim Lesen } \\
\text { oder Fernsehen)? }\end{array}$ & & & & & \\
\hline 32. & $\begin{array}{l}\text {...das Gefühl gehabt, dass Sie ein schlechtes } \\
\text { Gedächtnis hätten? }\end{array}$ & & & & & \\
\hline 33. & ...schlechte Träume oder Halluzinationen gehabt? & & & & & \\
\hline 34. & ...Schwierigkeiten mit dem Sprechen gehabt? & & & & & \\
\hline 35. & $\begin{array}{l}\text {...sich außer Stande gefühlt, mit anderen richtig zu } \\
\text { kommunizieren? }\end{array}$ & & & & & \\
\hline 36. & $\begin{array}{l}\text {...den Eindruck gehabt, von anderen nicht beachtet zu } \\
\text { werden? }\end{array}$ & & & & & \\
\hline 37. & ...schmerzhafte Muskelkrämpfe gehabt? & & & & & \\
\hline 38. & $\begin{array}{l}\text {...Schmerzen in den Gelenken oder anderen } \\
\text { Körperteilen gehabt? }\end{array}$ & & & & & \\
\hline 39. & ...sich unangenehm heiß oder kalt gefühlt? & & & & & \\
\hline
\end{tabular}

Fortsetzung Abbildung 6-6: Parkinson's Disease Questionnaire (PDQ-39), Fragen 26-39/39 


\section{Bitte kreuzen Sie die für Sie zutreffende Antwort zu jeder Frage an.}

1. Ich habe teilweise sehr lebhafte Träume.

2. Meine Träume haben des öfteren aggressiven oder aktionsgeladenen Inhalt.

3. Die Trauminhalte stimmen meist mit meinem nächtlichen Verhalten überein.

4. Mir ist bekannt, dass ich meine Arme oder Beine im Schlaf bewege.

5. Es ist dabei vorgekommen, dass ich meinen Partner oder mich selbst (beinahe) verletzt habe.

6. Bei mir treten oder traten während des Träumens folgende Erscheinungen auf:

6.1 laut Sprechen, Schreien, Schimpfen, Lachen

6.2 plötzliche Bewegungen der Gliedmaßen, „Kämpfen“

6.3 Gesten, Bewegungsabläufe, die im Schlaf sinnlos sind wie z.B. winken, salutieren, Mücken verscheuchen, Stürze aus dem Bett

6.4 um das Bett herum umgefallene Gegenstände wie z.B. Nachttischlampe, Buch, Brille

7. Es kommt vor, dass ich durch meine eigenen Bewegungen wach werde.

8. Nach dem Erwachen kann ich mich an den Inhalt meiner Träume meist gut erinnern.

9. Mein Schlaf ist häufiger gestört.

10. Bei mir liegt/lag eine Erkrankung des Nervensystems vor (z.B. Schlaganfall, Gehirnerschütterung, Parkinson, RLS, Narkolepsie, Depression, Epilepsie, entzündliche Erkrankung des Gehirns), welche?
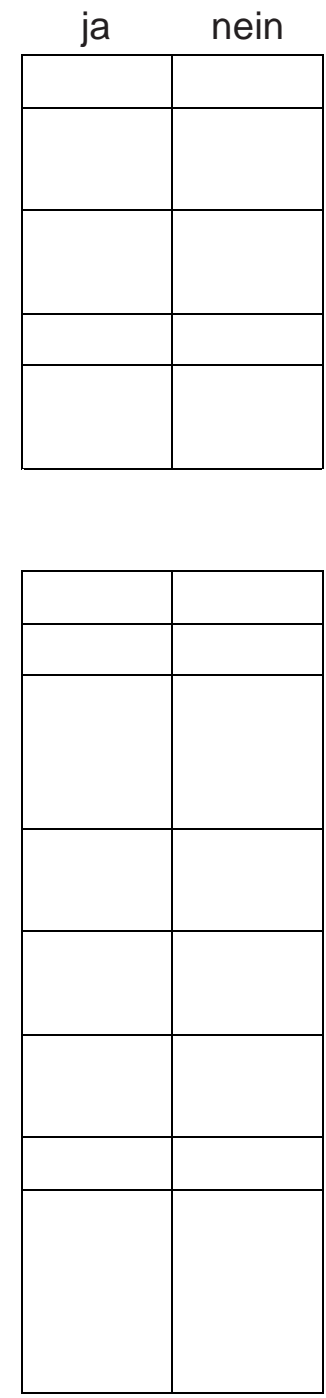

\section{Abbildung 6-7: RBD Screening Questionnaire (RBDSQ)} (RBD: REM-Schlaf-Verhaltensstörung; REM-Schlaf: Schlafphase mit schnellen Augenbewegungen) 


\section{LITERATURVERZEICHNIS}

Umlaute (ä, ö, ü) wurden wie die zugehörigen Vokale (a, o, u) sortiert.

Namenszusätze wie „van“, „de“, „Mc" oder „O" wurden bei der alphabetischen Reihung berücksichtigt.

Aarsland D, Andersen K, Larsen JP, Lolk A, Kragh-Sørensen P (2003): Prevalence and characteristics of dementia in Parkinson disease: an 8-year prospective study. Arch Neurol $\underline{60}, 387-392$

Aarsland D, Beyer MK, Kurz MW (2008): Dementia in Parkinson's disease. Curr Opin Neurol $\underline{2}, 676-682$

Aarsland D, Brønnick K, Alves G, Tysnes OB, Pedersen KF, Ehrt U, Larsen JP (2009 a): The spectrum of neuropsychiatric symptoms in patients with early untreated Parkinson's disease. J Neurol Neurosurg Psychiatry 80, 928-930

Aarsland D, Brønnick K, Larsen JP, Tysnes OB, Alves G, Norwegian ParkWest Study Group (2009 b): Cognitive impairment in incident, untreated Parkinson disease: The Norwegian ParkWest Study. Neurology $\underline{72}, 1121-1126$

Albin R, Young A, Penney J (1989): The functional anatomy of basal ganglia disorders. Trends Neurosci $\underline{12}, 366-375$

Alessandro S, Ceravolo R, Brusa L, Pierantozzi M, Costa A, Galati S, Placidi F, Romigi A, Iani C, Marzetti F et al. (2010): Non-motor functions in parkinsonian patients implanted in the pedunculopontine nucleus: focus on sleep and cognitive domains." J Neurol Sci 289, 44-48

Alves G, Forsaa EB, Pedersen KF, Dreetz Gjerstad M, Larsen JP (2008): Epidemiology of Parkinson's disease. J Neurol 255, 18-32

Alves G, Brønnick K, Aarsland D, Blennow K, Zetterberg H, Ballard C, Kurz MW, Andreasson U, Tysnes OB, Larsen JP et al. (2010): CSF amyloid- $ß$ and tau proteins, and cognitive performance, in early and untreated Parkinson's Disease: the Norwegian ParkWest study. J Neurol Neurosurg Psychiatry $\underline{81}, 1080-1086$

American Academy of Sleep Medicine: The international classification of sleep disorders, revised: Diagnostic and coding manual. American Academy of Sleep Medicine, Chicago 2001. URL: http://www.esst.org/adds/ICSD.pdf [letzter Abruf: 23.03.2012]

Ansari K (1975): Olfactory function in patients with Parkinson's disease. J Chron Dis $\underline{28}$, 493-497

Aranda B, Cramer P (1993): Effects of apomorphine and L-dopa on the parkinsonian bladder. Neurourol Urodyn 12, 203-209

Arvanitakis Z, Wilson RS, Schneider JA, Bienias JL, Evans DA, Bennett DA (2004): Diabetes mellitus and progression of rigidity and gait disturbance in older persons. Neurology $\underline{63}, 996-1001$

Ascherio A, Chen H, Weisskopf MG, O'Reilly E, Mc Cullough ML, Calle EE, Schwarzschild MA, Thun MJ (2006): Pesticide exposure and risk for Parkinson's disease. Ann Neurol $60,197-203$

Awerbuch GI, Sandyk R (1992): Autonomic functions in the early stages of Parkinson's disease. Int J Neurosci 64, 7-14

Barone P, Antonini A, Colosimo C, Marconi R, Morgante L, Avarello TP, Bottacchi E, Cannas A, Ceravolo G, Ceravolo R et al. (2009): The PRIAMO study: A multicenter assessment of nonmotor symptoms and their impact on quality of life in Parkinson's disease. Mov Disord 24, 1641-1649

Barrett MJ, Wylie SA, Harrison MB, Wooten GF (2011): Handedness and motor symptom asymmetry in Parkinson's disease. J Neurol Neurosurg Psychiatry $\underline{82}, 1122-1124$ 
Becker G, Seufert J, Bogdahn U, Reichmann H, Reiners K (1995): Degeneration of substantia nigra in chronic Parkinson's disease visualized by transcranial color-coded real-time sonography. Neurology $\underline{45}, 182-184$

Becker G, Müller A, Braune S, Büttner T, Benecke R, Greulich W, Klein W, Mark G, Rieke J, Thümler R (2002): Early diagnosis of Parkinson's disease. J Neurol 249 (Suppl. 3): III/40-III/48

Benson GS, Raezer DM, Anderson JR, Saunders CD, Corriere JN (1976): Effect of levodopa on urinary bladder. Urology $\underline{7}, 24-28$

Berendse HW, Ponsen MM (2009): Diagnosing premotor Parkinson's disease using a twostep approach combining olfactory testing and DAT SPECT imaging. Parkinsonism Relat Disord 15 (Suppl. 3), S26-S30

Berendse HW, Booij J, Francot CM, Bergmans PL, Hijman R, Stoof JC, Wolters EC (2001): Subclinical dopaminergic dysfunction in asymptomatic Parkinson's disease patients' relatives with a decreased sense of smell. Ann Neurol $\underline{50}, 34-41$

Berg D, Becker G, Zeiler B, Tucha O, Hofmann E, Preier M, Benz P, Jost W, Reiners K, Lange KW (1999 a): Vulnerability of the nigrostriatal system as detected by transcranial ultrasound. Neurology 53, 1026-1031

Berg D, Grote C, Rausch WD, Mäurer M, Wesemann W, Riederer P, Becker G (1999 b): Iron accumulation in the substantia nigra in rats visualized by ultrasound. Ultrasound Med Biol 25, 901-904

Berg D, Siefker C, Becker G (2001 a): Echogenicity of the substantia nigra in Parkinson's disease and its relation to clinical findings. J Neurol 248, 684-689

Berg D, Siefker C, Ruprecht-Dörfler P, Becker G (2001 b): Relationship of substantia nigra echogenicity and motor function in elderly subjects. Neurology $56,13-17$

Berg D, Merz B, Reiners K, Naumann M, Becker G (2005): Five-year follow-up study of hyperechogenicity of the substantia nigra in Parkinson's disease. Mov Disord $\underline{20}$, 383385

Berger K, Broll S, Winkelmann J, Heberlein I, Müller T, Ries V (1999): Untersuchung zur Reliabilität der deutschen Version des PDQ-39: Ein krankheitsspezifischer Fragebogen zur Erfassung der Lebensqualität von Parkinson-Patienten. Akt Neurol 26, 180-184

Bernheimer H, Birkmayer W, Hornykiewicz O, Jellinger K, Seitelberger F (1973): Brain dopamine and the syndromes of Parkinson and Huntington Clinical, morphological and neurochemical correlations. J Neurol Sci 20, 415-455

Binder LI, Frankfurter A, Rebhun LI (1985): The distribution of tau in the mammalian central nervous system. J Cell Biol 101, 1371-1378

Biomarkers Definitions Working Group (2001): Biomarkers and surrogate endpoints: Preferred definitions and conceptual framework. Clin Pharmacol Ther $\underline{69}$, 89-95

Blennow K (2004): Cerebrospinal Fluid Protein Biomarkers for Alzheimer's Disease. NeuroRx 1, 213-225

Boesveldt S, Verbaan D, Knol DL, Visser M, van Rooden SM, van Hilten JJ, Berendse HW (2008): A comparative study of odor identification and odor discrimination deficits in Parkinson's disease. Mov Disord 23, 1984-1990

Boeve BF (2004): REM Sleep Behavior Disorder in Parkinson's Disease and Dementia with Lewy Bodies. J Geriatr Psychiatry Neurol 17, 146-157

Bogdanov M, Matson WR, Wang L, Matson T, Saunders-Pullman R, Bressman SS, Flint Beal M (2008): Metabolomic profiling to develop blood biomarkers for Parkinson's disease. Brain 131, 389-396

Bohnen NI, Studenski SA, Constantine GM, Moore RY (2008): Diagnostic performance of clinical motor and non-motor tests of Parkinson disease: a matched case-control study. Eur J Neurol $\underline{15}$, 685-691 
Bonuccelli U, Lucetti C, Del Dotto P, Ceravolo R, Gambaccini G, Bernardini S, Rossi G, Piaggesi A (2003): Orthostatic hypotension in de novo Parkinson disease. Arch Neurol 60, 1400-1404

Braak H (2003): Staging of brain pathology related to sporadic Parkinson's disease. Neurobiol Aging 24, 197-211

Braak H, Del Tredici K (2010): Pathophysiologie des sporadischen Morbus Parkinson. Fortschr Neurol Psychiatr 78 (Suppl. 1), S2-S4

Braune S (2001): The role of cardiac metaiodobenzylguanidine uptake in the differential diagnosis of parkinsonian syndromes. Clin Auton Res 11, 351-355

Brodaty H, Moore CM (1997): The Clock Drawing Test for dementia of the Alzheimer's type: A comparison of three scoring methods in a memory disorders clinic. Int J Geriatr Psychiatry $\underline{12}, 619-627$

Bronnick K, Emre M, Lane R, Tekin S, Aarsland D (2007): Profile of cognitive impairment in dementia associated with Parkinson's disease compared with Alzheimer's disease. J Neurol Neurosurg Psychiatry $\underline{78}, 1064-1068$

Brooks DJ, Frey KA, Marek KL, Oakes D, Paty D, Prentice R, Shults CW, Stoessl AJ (2003): Assessment of neuroimaging techniques as biomarkers of the progression of Parkinson's disease. Exp Neurol 184 (Suppl. 1), S68-S79

Brozova H, Stochl J, Roth J, Ruzicka E (2009): Fear of falling has greater influence than other aspects of gait disorders on quality of life in patients with Parkinson's disease. Neuro Endocrinol Lett 30, 453-457

Burn DJ (2006): Parkinson's disease dementia: what's in a Lewy body? J Neural Transm $\underline{70}$ (Suppl.), 361-365

Büttner T, Kuhn W, Müller T, Patzold T, Heidbrink K, Przuntek H (1995): Distorted color discrimination in 'de novo' parkinsonian patients. Neurology $\underline{45}, 386-387$

Calne DB, Brennan J, Spiers AS, Stern GM (1970): Hypotension caused by L-dopa. Br Med J 5694, 474-475

Campbell N, Boustani M, Limbil T, Ott C, Fox C, Maidment I, Schubert CC, Munger S, Fick $D$, Miller D et al. (2009): The cognitive impact of anticholinergics: A clinical review. Clin Interv Aging 4, 225-233

Chastan N, Debono B, Maltête D, Weber J (2008): Discordance between measured postural instability and absence of clinical symptoms in Parkinson's disease patients in the early stages of the disease. Mov Disord 23, 366-372

Chaudhuri KR, Healy DG, Schapira AHV (2006 a): Non-motor symptoms of Parkinson's disease: diagnosis and management. Lancet Neurol $\underline{5}, 235-245$

Chaudhuri KR, Martinez-Martin P, Schapira AH, Stocchi F, Sethi K, Odin P, Brown RG, Koller W, Barone P, MacPhee G et al. (2006 b): International multicenter pilot study of the first comprehensive self-completed nonmotor symptoms questionnaire for Parkinson's disease: The NMSQuest study. Mov Disord 21, 916-923

Chaudhuri KR, Martinez-Martin P, Brown RG, Sethi K, Stocchi F, Odin P, Ondo W, Abe K, Macphee G, Macmahon D et al. (2007): The metric properties of a novel non-motor symptoms scale for Parkinson's disease: Results from an international pilot study. Mov Disord 22, 1901-1911

Clayton LM, Naidu Y, Odin P, Martinez P, Sethi K, Schapira A, Bonucelli U, Stocchi F, Rabey M, MacMahon D et al. (2006): Comparison of the prevalence and pattern of non-motor symptoms in Parkinson's disease in drug naïve and treated patients using the NMSQuest. Mov Disord 21 (Suppl. 15), S648

Clinton LK, Blurton-Jones M, Myczek K, Trojanowski JQ, LaFerla FM (2010): Synergistic Interactions between $A \beta$, tau, and $\alpha$-synuclein: acceleration of neuropathology and cognitive decline. J Neurosci $\underline{30}, 7281-7289$

Compta Y, Martí MJ, Ibarretxe-Bilbao N, Junqué C, Valldeoriola F, Muñoz E, Ezquerra M, Ríos J, Tolosa E (2009): Cerebrospinal tau, phospho-tau, and beta-amyloid and neuropsychological functions in Parkinson's disease. Mov Disord 24, 2203-2210 
Cooper JA, Sagar HJ, Jordan N, Harvey NS, Sullivan EV (1991): Cognitive impairment in early, untreated Parkinson's disease and its relationship to motor disability. Brain $\underline{114}$, 2095-2122

Crum RM, Anthony JC, Bassett SS, Folstein MF (1993): Population-based norms for the Mini-Mental State Examination by age and educational level. JAMA 269, 2386-2391

Damier P, Hirsch EC, Agid Y, Graybiel AM (1999): The substantia nigra of the human brain. Brain 122, 1437-1448

Daum RF, Sekinger B, Kobal G, Lang CJG (2000): Riechprüfung mit "sniffin' sticks" zur klinischen Diagnostik des Morbus Parkinson. Nervenarzt $\underline{71}$, 643-650

den Hartog Jager WA, Bethlem J (1960): The distribution of Lewy bodies in the central and autonomic nervous systems in idiopathic paralysis agitans. J Neurol Neurosurg Psychiatry $\underline{23}, 283-290$

de Rijk MC, Tzourio C, Breteler MM, Dartigues JF, Amaducci L, Lopez-Pousa S, ManubensBertran JM, Alpérovitch A, Rocca WA (1997): Prevalence of parkinsonism and Parkinson's disease in Europe: the EUROPARKINSON Collaborative Study. European Community Concerted Action on the Epidemiology of Parkinson's disease. J Neurol Neurosurg Psychiatry 62, 10-15.

Dexter DT, Sian J, Rose S, Hindmarsh JG, Mann VM, Cooper JM, Wells FR, Daniel SE, Lees AJ, Schapira AH (1994): Indices of oxidative stress and mitochondrial function in individuals with incidental Lewy body disease. Ann Neurol 35, 38-44

Dick FD, De Palma G, Ahmadi A, Scott NW, Prescott GJ, Bennett J, Semple S, Dick S, Counsell C, Mozzoni $P$ et al. (2007): Environmental risk factors for Parkinson's disease and parkinsonism: the Geoparkinson study. Occup Environ Med 64, 666-672

Doty RL, Deems DA, Stellar S (1988): Olfactory dysfunction in parkinsonism: a general deficit unrelated to neurologic signs, disease stage, or disease duration. Neurology $\underline{38}, 1237-1244$

Doty RL, Stern MB, Pfeiffer C, Gollomp SM, Hurtig HI (1992): Bilateral olfactory dysfunction in early stage treated and untreated idiopathic Parkinson's disease. J Neurol Neurosurg Psychiatry $\underline{55}, 138-142$

Driver JA, Logroscino G, Gaziano JM, Kurth T (2009): Incidence and remaining lifetime risk of Parkinson disease in advanced age. Neurology $\underline{72}, 432-438$

Dubois B, Burn D, Goetz C, Aarsland D, Brown RG, Broe GA, Dickson D, Duyckaerts C, Cummings J, Gauthier S et al. (2007 a): Diagnostic procedures for Parkinson's disease dementia: recommendations from the movement disorder society task force. Mov Disord 22, 2314-2324

Dubois B, Feldman HH, Jacova C, Dekosky ST, Barberger-Gateau P, Cummings J, Delacourte A, Galasko D, Gauthier S, Jicha G (2007 b): Research criteria for the diagnosis of Alzheimer's disease: revising the NINCDS-ADRDA criteria." Lancet Neurol $\underline{6}, 734-746$.

Eisensehr I, Linke R, Noachtar S, Schwarz J, Gildehaus FJ, Tatsch K (2000): Reduced striatal dopamine transporters in idiopathic rapid eye movement sleep behaviour disorder. Comparison with Parkinson's disease and controls. Brain $\underline{123}, 1155-1160$

Eisensehr I, v Lindeiner H, Jäger M, Noachtar S (2001): REM sleep behavior disorder in sleep-disordered patients with versus without Parkinson's disease: is there a need for polysomnography? J Neurol Sci 186, 7-11

Elbaz A, Bower JH, Peterson BJ, Maraganore DM, Mc Donnell SK, Ahlskog JE, Schaid DJ, Rocca WA (2003): Survival study of Parkinson disease in Olmsted County, Minnesota. Arch Neurol 60, 91-96

Elgh E, Domellöf M, Linder J, Edström M, Stenlund H, Forsgren L (2009): Cognitive function in early Parkinson's disease: a population-based study. Eur J Neurol 16, 1278-1284

Emre M, Aarsland D, Albanese A, Byrne EJ, Deuschl G, De Deyn PP, Durif F, Kulisevsky J, van Laar T, Lees A et al. (2004): Rivastigmine for dementia associated with Parkinson's disease. N Engl J Med 351, 2509-2518 
Emre M, Aarsland D, Brown R, Burn DJ, Duyckaerts C, Mizuno Y, Broe GA, Cummings J, Dickson DW, Gauthier S et al. (2007): Clinical diagnostic criteria for dementia associated with Parkinson's disease. Mov Disord 22, 1689-1707

Fahn S, Elton RL, UPDRS program members: Unified Parkinson's Disease Rating Scale; in: Recent developments in Parkinson's disease, vol. 2; hrsg. von Fahn S, Marsden CD, Goldstein M, Calne DB; Macmillan Healthcare Information, Florham Park 1987, 153163

Fall PA, Fredrikson M, Axelson O, Granérus AK (1999): Nutritional and occupational factors influencing the risk of Parkinson's disease: a case-control study in southeastern Sweden. Mov Disord 14, 28-37

Fearnley JM, Lees AJ (1991): Ageing and Parkinson's disease: substantia nigra regional selectivity. Brain 114, 2283-2301

Filipović SR, Sternić N, Svetel M, Dragasević N, Lecic D, Kostić VS (2001):

Bereitschaftspotential in depressed and non-depressed patients with Parkinson's disease. Mov Disord 16, 294-300

Fitzpatrick R, Peto V, Jenkinson C, Greenhall R, Hyman N (1997): Health-related quality of life in Parkinson's disease: a study of outpatient clinic attenders. Mov Disord 12, $916-$ 922

Folstein M (1975): "Mini-mental state": A practical method for grading the cognitive state of patients for the clinician. J Psychiatr Res 12, 189-198

Forsaa EB, Larsen JP, Wentzel-Larsen T, Goetz CG, Stebbins GT, Aarsland D, Alves G (2010): A 12-year population-based study of psychosis in Parkinson disease. Arch Neurol 67, 996-1001

Gaenslen A, Unmuth B, Godau J, Liepelt I, Di Santo A, Schweitzer KJ, Gasser T, Machulla $\mathrm{H}$, Reimold M, Marek K et al. (2008): The specificity and sensitivity of transcranial ultrasound in the differential diagnosis of Parkinson's disease: a prospective blinded study. Lancet Neurol $\underline{7}, 417-424$

Gagnon JF, Bédard MA, Fantini ML, Petit D, Panisset M, Rompré S, Carrier J, Montplaisir J (2002): REM sleep behavior disorder and REM sleep without atonia in Parkinson's disease. Neurology $\underline{59}, 585-589$

Gelb DJ, Oliver E, Gilman S (1999): Diagnostic Criteria for Parkinson Disease. Arch Neurol $\underline{56}, 33-39$

Gibb WR, Lees AJ (1988): The relevance of the Lewy body to the pathogenesis of idiopathic Parkinson's disease. J Neurol Neurosurg Psychiatry $\underline{\text { 51, 745-752 }}$

Gjerstad MD, Boeve B, Wentzel-Larsen T, Aarsland D, Larsen JP (2008): Occurrence and clinical correlates of REM sleep behaviour disorder in patients with Parkinson's disease over time. J Neurol Neurosurg Psychiatry $\underline{79}$, 387-391

Goetz CG, Chmura TA, Lanska DJ (2001): The history of Parkinson's disease: part 2 of the MDS-sponsored History of Movement Disorders Exhibit, Barcelona, June, 2000. Mov Disord 16, 156-161

Goetz CG, Poewe W, Rascol O, Sampaio C, Stebbins GT, Counsell C, Giladi N, Holloway RG, Moore CG, Wenning GK et al. (2004): Movement Disorder Society Task Force report on the Hoehn and Yahr staging scale: Status and recommendations. Mov Disord 19, 1020-1028

Goetz CG, Ouyang B, Negron A, Stebbins GT (2010): Hallucinations and sleep disorders in PD: ten-year prospective longitudinal study. Neurology $\underline{75}, 1773-1779$

Goldstein DS (2006): Orthostatic hypotension as an early finding in Parkinson's disease. Clin Auton Res 16, 46-54

Grimmer T, Riemenschneider M, Förstl H, Henriksen G, Klunk WE, Mathis CA, Shiga T, Wester H, Kurz A, Drzezga A (2009): Beta Amyloid in Alzheimer's Disease: Increased Deposition in Brain Is Reflected in Reduced Concentration in Cerebrospinal Fluid.

Biol Psychiatry $\underline{65}$, 927-934 
Grünblatt E, Zehetmayer S, Jacob CP, Müller T, Jost WH, Riederer P (2010): Pilot study: peripheral biomarkers for diagnosing sporadic Parkinson's disease. J Neural Transm $117,1387-1393$

Haass C, Selkoe DJ (1993): Cellular processing of $\beta$-amyloid precursor protein and the genesis of amyloid $\beta$-peptide. Cell $\underline{75}, 1039-1042$

Haaxma CA, Bloem BR, Overeem S, Borm GF, Horstink MWIM (2010): Timed motor tests can detect subtle motor dysfunction in early Parkinson's disease. Mov Disord $\underline{25}$, 1150-1156

Haehner A, Boesveldt S, Berendse HW, Mackay-Sim A, Fleischmann J, Silburn PA, Johnston AN, Mellick GD, Herting B, Reichmann H et al. (2009 a): Prevalence of smell loss in Parkinson's disease - a multicenter study. Parkinsonism Relat Disord $\underline{15}$, 490-494

Haehner A, Hummel T, Reichmann H (2009 b): Olfactory dysfunction as a diagnostic marker for Parkinson's disease. Expert Rev Neurother $\underline{9}$, 1773-1779

Halliday G, Hely M, Reid W, Morris J (2008): The progression of pathology in longitudinally followed patients with Parkinson's disease. Acta Neuropathol 115, 409-415

Hampel H, Buerger K, Zinkowski R, Teipel SJ, Goernitz A, Andreasen N, Sjoegren M, DeBernardis J, Kerkman D, Ishiguro K et al. (2004): Measurement of phosphorylated tau epitopes in the differential diagnosis of Alzheimer disease: a comparative cerebrospinal fluid study. Arch Gen Psychiatry 61, 95-102

Hariz G, Forsgren L (2011): Activities of daily living and quality of life in persons with newly diagnosed Parkinson's disease according to subtype of disease, and in comparison to healthy controls. Acta Neurol Scand $\underline{123}, 20-27$

Hardie RJ, Efthimiou J, Stern GM (1986): Respiration and sleep in Parkinson's disease. J Neurol Neurosurg Psychiatry $\underline{49}, 1326$

Hawkes $\mathrm{CH}$, Shephard BC, Daniel SE (1997): Olfactory dysfunction in Parkinson's disease. $\mathrm{J}$ Neurol Neurosurg Psychiatry $\underline{62}, 436-446$

Hely MA, Morris JG, Reid WG, Trafficante R (2005): Sydney multicenter study of Parkinson's disease: Non-L-dopa-responsive problems dominate at 15 years. Mov Disord $\underline{20}$, 190-199

Hely MA, Reid WGJ, Adena MA, Halliday GM, Morris JGL (2008): The Sydney multicenter study of Parkinson's disease: the inevitability of dementia at 20 years. Mov Disord $\underline{23}$, 837-844

Henningsen $\mathrm{H}$ : Morbus Parkinson im Wandel medizinischer Krankheitsvorstellungen; in: Pathophysiologie, Klinik und Therapie des Parkinsonismus; hrsg. v. Gänshirt H unter Mitarbeit von Berlit P und Haack G; Editiones Roche, Basel 1983, 93-101

Hesse C, Rosengren L, Vanmechelen E, Vanderstichele H, Jensen C, Davidsson P, Blennow K (2000): Cerebrospinal fluid markers for Alzheimer's disease evaluated after acute ischemic stroke. J Alzheimers Dis $\underline{2}$, 199-206

Hesse C, Rosengren L, Andreasen N, Davidsson P, Vanderstichele H, Vanmechelen E, Blennow K (2001): Transient increase in total tau but not phospho-tau in human cerebrospinal fluid after acute stroke. Neurosci Lett 297, 187-190

Hoehn MM, Yahr MD (1967): Parkinsonism: onset, progression, and mortality. Neurology $\underline{17}$, 427-442

Holmberg B, Johnels B, Blennow K, Rosengren $L$ (2003): Cerebrospinal fluid $A \beta 42$ is reduced in multiple system atrophy but normal in Parkinson's disease and progressive supranuclear palsy. Mov Disord 18, 186-190

Honig $\mathrm{H}$, Antonini A, Martinez-Martin P, Forgacs I, Faye GC, Fox T, Fox K, Mancini F, Canesi M, Odin P et al. (2009): Intrajejunal levodopa infusion in Parkinson's disease: a pilot multicenter study of effects on nonmotor symptoms and quality of life. Mov Disord 24, 1468-1474

Hughes AJ, Lees AJ, Stern GM (1990): Apomorphine test to predict dopaminergic responsiveness in parkinsonian syndromes. Lancet $\underline{336}, 32-34$ 
Hughes AJ, Daniel SE, Kilford L, Lees AJ (1992): Accuracy of clinical diagnosis of idiopathic Parkinson's disease: a clinico-pathological study of 100 cases. J Neurol Neurosurg Psychiatry $\underline{55}, 181-184$

Hughes AJ, Daniel SE, Lees AJ (2001): Improved accuracy of clinical diagnosis of Lewy body Parkinson's disease. Neurology 57, 1497-1499

Hughes AJ, Daniel SE, Ben-Shlomo Y, Lees AJ (2002): The accuracy of diagnosis of parkinsonian syndromes in a specialist movement disorder service. Brain $\underline{125}$, 861870

Hummel T, Sekinger B, Wolf S, Pauli E, Kobal G (1997): 'Sniffin' Sticks': Olfactory Performance Assessed by the Combined Testing of Odor Identification, Odor Discrimination and Olfactory Threshold. Chem Senses 22, 39-52

Hurtig HI, Trojanowski JQ, Galvin J, Ewbank D, Schmidt ML, Lee VM, Clark CM, Glosser G, Stern MB, Gollomp SM et al. (2000): Alpha-synuclein cortical Lewy bodies correlate with dementia in Parkinson's disease. Neurology $\underline{54}, 1916-1921$

Iber C, Ancoli-Israel S, Chesson AL, Quan SF for the American Academy of Sleep Medicine: The AASM Manual for the Scoring of Sleep and Associated Events: Rules, Terminology and Technical Specifications. American Academy of Sleep Medicine, Westchester 2007

Iqbal K, Grundke-Iqbal I, Zaidi T, Merz PA, Wen GY, Shaikh SS, Wisniewski HM, Alafuzoff I, Winblad B (1986): Defective brain microtubule assembly in Alzheimer's disease. Lancet 328, 421-426

Iranzo A, Santamaría J, Rye DB, Valldeoriola F, Martí MJ, Muñoz E, Vilaseca I, Tolosa E (2005): Characteristics of idiopathic REM sleep behavior disorder and that associated with MSA and PD. Neurology $65,247-252$

Ishihara L, Brayne C (2006): A systematic review of depression and mental illness preceding Parkinson's disease. Acta Neurol Scand 113, 211-220

Iwai A, Masliah E, Yoshimoto M, Ge N, Flanagan L, de Silva HA, Kittel A, Saitoh T (1995): The precursor protein of non-Aß component of Alzheimer's disease amyloid is a presynaptic protein of the central nervous system. Neuron 14, 467-475

Iwatsubo T, Odaka A, Suzuki N, Mizusawa H, Nukina N, Ihara Y (1994): Visualization of A beta 42(43) and $A$ beta 40 in senile plaques with end-specific $A$ beta monoclonals: evidence that an initially deposited species is A beta 42(43). Neuron $\underline{13}, 45-53$

Jankovic J (2008): Parkinson's disease: clinical features and diagnosis. J Neurol Neurosurg Psychiatry $\underline{79}, 368-376$

Jankovic J, Mc Dermott M, Carter J, Gauthier S, Goetz C, Golbe L, Huber S, Koller W, Olanow C, Shoulson I et al. (1990): Variable expression of Parkinson's disease: a base-line analysis of the DATATOP cohort. Neurology $\underline{40}$, 1529-1534

Jankovic J, Rajput AH, Mc Dermott MP, Perl DP (2000): The evolution of diagnosis in early Parkinson disease. Arch Neurol 57, 369-372

Jellinger K (1987): Quantitative changes in some subcortical nuclei in aging, Alzheimer's disease and Parkinson's disease. Neurobiol Aging $\underline{8}, 556-561$

Jellinger KA (2003); Neuropathological spectrum of synucleinopathies. Mov Disord $\underline{18}, 2-12$

Jellinger KA, Seppi K, Wenning GK, Poewe W (2002): Impact of coexistent Alzheimer pathology on the natural history of Parkinson's disease. J Neural Transm $\underline{109}$, 329339

Kandiah N, Narasimhalu K, Lau P, Seah S, Au WL, Tan LCS (2009): Cognitive decline in early Parkinson's disease. Mov Disord 24, 605-608

Kanemaru K, Kameda N, Yamanouchi H (2000): Decreased CSF amyloid beta42 and normal tau levels in dementia with Lewy bodies. Neurology 54, 1875-1876

Katotomichelakis M, Balatsouras D, Tripsianis G, Davris S, Maroudias N, Danielides V, Simopoulos C (2007): The effect of smoking on the olfactory function. Rhinology $\underline{45}$, 273-280 
Kaufmann H, Nahm K, Purohit D, Wolfe D (2004): Autonomic failure as the initial presentation of Parkinson disease and dementia with Lewy bodies. Neurology $\underline{63}$, 1093-1095

Kessler J, Markowitsch HJ, Denzler PE: MMST: Mini-Mental-Status-Test. Deutschsprachige Fassung. Beltz, Weinheim 1990

Klawans HL (1986): Individual manifestations of Parkinson's disease after ten or more years of levodopa. Mov Disord 1, 187-192

Kobal G, Klimek L, Wolfensberger M, Gudziol H, Temmel A, Owen CM, Seeber H, Pauli E, Hummel T (2000): Multicenter investigation of 1,036 subjects using a standardized method for the assessment of olfactory function combining tests of odor identification, odor discrimination, and olfactory thresholds. Eur Arch Otorhinolaryngol 257, 205-211

Korchounov A, Schipper HI, Preobrazhenskaya IS, Kessler KR, Yakhno NN (2004): Differences in age at onset and familial aggregation between clinical types of idiopathic Parkinson's disease. Mov Disord 19, 1059-1064

Kramer ML, Schulz-Schaeffer WJ (2007): Presynaptic a-synuclein aggregates, not Lewy bodies, cause neurodegeneration in dementia with Lewy bodies. J Neurosci 27, 14051410

Krishnan PR, Bhatia M, Behari M (2003): Restless legs syndrome in Parkinson's disease: a case-controlled study. Mov Disord 18, 181-185

Kujawa K, Leurgans S, Raman R, Blasucci L, Goetz CG (2000): Acute orthostatic hypotension when starting dopamine agonists in Parkinson's disease. Arch Neurol $\underline{57}$, 1461-1463

Kurz M, Alves G, Aarsland D, Larsen JP (2003): Familial Parkinson's disease: a communitybased study. Eur J Neurol 10, 159-163

Langston JW (2006): The Parkinson's complex: parkinsonism is just the tip of the iceberg. Ann Neurol 59, 591-596

Lashley T, Holton JL, Gray E, Kirkham K, O'Sullivan SS, Hilbig A, Wood NW, Lees AJ, Revesz T (2008): Cortical alpha-synuclein load is associated with amyloid-beta plaque burden in a subset of Parkinson's disease patients. Acta Neuropathol $\underline{115}$, 417-425

Lee CS, Schulzer M, Mak E, Hammerstad JP, Calne S, Calne DB (1995): Patterns of asymmetry do not change over the course of idiopathic parkinsonism: implications for pathogenesis. Neurology 45 , 435-439

Lees AJ, Hardy J, Revesz T (2009): Parkinson's disease. Lancet 373, 2055-2066

Lewczuk P, Esselmann H, Bibl M, Beck G, Maler JM, Otto M, Kornhuber J, Wiltfang J (2004): Tau protein phosphorylated at threonine 181 in CSF as a neurochemical biomarker in Alzheimer's disease: original data and review of the literature. $\mathrm{J} \mathrm{Mol}$ Neurosci $23,115-122$

Lewy FH (1913): Zur pathologischen Anatomie der Paralysis agitans. Dtsch Z Nervenh 50, 50-55. URL: http://www2.psykl.med.tum.de/geschichte_history/Lewy-paralysisagitans-body.html [letzter Abruf: 23.03.2012]

Louis ED, Klatka LA, Liu Y, Fahn S (1997): Comparison of extrapyramidal features in 31 pathologically confirmed cases of diffuse Lewy body disease and 34 pathologically confirmed cases of Parkinson's disease. Neurology 48, 376-380

Low PA (2008): Prevalence of orthostatic hypotension. Clin Auton Res 18 (Suppl. 1), 8-13

Mamikonyan E, Moberg PJ, Siderowf A, Duda JE, Have TT, Hurtig HI, Stern MB, Weintraub $D$ (2009): Mild cognitive impairment is common in Parkinson's disease patients with normal Mini-Mental State Examination (MMSE) scores. Parkinsonism Relat Disord $15,226-231$

Mandelkow EM, Mandelkow E (1994): Tau protein and Alzheimer's disease. Neurobiol Aging 15 (Suppl. 2), S85-S86

Marsden CD (1994): Parkinson's disease. J Neurol Neurosurg Psychiatry $\underline{57}$, 672-681 
Martinez-Martin P, Schapira AH, Stocchi F, Sethi K, Odin P, MacPhee G, Brown RG, Naidu $\mathrm{Y}$, Clayton L, Abe K et al. (2007): Prevalence of nonmotor symptoms in Parkinson's disease in an international setting; Study using nonmotor symptoms questionnaire in 545 patients. Mov Disord 22, 1623-1629

Martinez-Martin P, Rodriguez-Blazquez C, Abe K, Bhattacharyya KB, Bloem BR, CarodArtal FJ, Prakash R, Esselink RAJ, Falup-Pecurariu C, Gallardo M et al. (2009): International study on the psychometric attributes of the non-motor symptoms scale in Parkinson disease. Neurology $\underline{73}, 1584-1591$

Mayeux R, Saunders AM, Shea S, Mirra S, Evans D, Roses AD, Hyman BT, Crain B, Tang MX, Phelps CH (1998): Utility of the apolipoprotein E genotype in the diagnosis of Alzheimer's disease. Alzheimer's Disease Centers Consortium on Apolipoprotein $E$ and Alzheimer's Disease. N Engl J Med 338, 506-511

Mc Naught KS, Shashidharan P, Perl DP, Jenner P, Olanow CW (2002): Aggresome-related biogenesis of Lewy bodies. Eur J Neurosci 16, 2136-2148

Mc Neill TH, Brown SA, Rafols JA, Shoulson I (1988): Atrophy of medium spiny I striatal dendrites in advanced Parkinson's disease. Brain Res 455, 148-152.

Meara J, Bhowmick BK, Hobson P (1999): Accuracy of diagnosis in patients with presumed Parkinson's disease. Age Ageing 28, 99-102

Mehnert S, Reuter I, Schepp K, Maaser P, Stolz E, Kaps M (2010): Transcranial sonography for diagnosis of Parkinson's disease. BMC Neurol 10 (9), 1-7

Merello M, Nouzeilles MI, Arce GP, Leiguarda R (2002): Accuracy of acute levodopa challenge for clinical prediction of sustained long-term levodopa response as a major criterion for idiopathic Parkinson's disease diagnosis. Mov Disord 17, 795-798

Michell AW, Lewis SJG, Foltynie T, Barker RA (2004): Biomarkers and Parkinson's disease. Brain 127, 1693-1705

Mollenhauer B, Trenkwalder C, von Ahsen N, Bibl M, Steinacker P, Brechlin P, Schindehuette J, Poser S, Wiltfang J, Otto M (2006): Beta-amlyoid 1-42 and tauprotein in cerebrospinal fluid of patients with Parkinson's disease dementia. Dement Geriatr Cogn Disord 22, 200-208

Mollenhauer B, Cullen V, Kahn I, Krastins B, Outeiro TF, Pepivani I, Ng J, Schulz-Schaeffer W, Kretzschmar HA, Mc Lean PJ et al. (2008): Direct quantification of CSF alphasynuclein by ELISA and first cross-sectional study in patients with neurodegeneration. Exp Neurol 213, 315-325

Molloy DW, Alemayehu E, Roberts R (1991): Reliability of a Standardized Mini-Mental State Examination compared with the traditional Mini-Mental State Examination. Am J Psychiatry $148,102-105$

Montgomery EB, Koller WC, LaMantia TJ, Newman MC, Swanson-Hyland E, Kaszniak AW, Lyons K (2000): Early detection of probable idiopathic Parkinson's disease: I. Development of a diagnostic test battery. Mov Disord Society 15 , 467-473

Moore P, Knowles D (2006): Beliefs and Knowledge about Parkinson's Disease. E J Appl Psychol 2, 15-21

Motter R, Vigo-Pelfrey C, Kholodenko D, Barbour R, Johnson-Wood K, Galasko D, Chang L, Miller B, Clark C, Green R (1995): Reduction of $\beta$-amyloid peptide42 in the cerebrospinal fluid of patients with Alzheimer's disease. Ann Neurol 38, 643-648

Movement Disorder Society Task Force on Rating Scales for Parkinson's Disease (2003): The Unified Parkinson's Disease Rating Scale (UPDRS): Status and recommendations. Mov Disord 18, 738-750

Müller A, Reichmann H, Livermore A, Hummel T (2002): Olfactory function in idiopathic Parkinson's disease (IPD): results from cross-sectional studies in IPD patients and long-term follow-up of de-novo IPD patients. J Neural Transm 109, 805-811

Murphy C, Schubert CR, Cruickshanks KJ, Klein BEK, Klein R, Nondahl DM (2002): Prevalence of olfactory impairment in older adults. JAMA 288, 2307-2312 
Muslimovic D, Post B, Speelman JD, Schmand B (2005): Cognitive profile of patients with newly diagnosed Parkinson disease. Neurology $\underline{65}, 1239-1245$.

Nomura T, Inoue Y, Miyake M, Yasui K, Nakashima K (2006): Prevalence and clinical characteristics of restless legs syndrome in Japanese patients with Parkinson's disease. Mov Disord 21, 380-384

Oertel WH, Reichmann H, Expertengruppe des Kompetenznetz Parkinson: Leitlinie Parkinson-Syndrome: Diagnostik und Therapie. Sonderauszugausgabe aus: Leitlinien für Diagnostik und Therapie in der Neurologie; hrsg. v. Diener HC, Putzki N und der Kommission Leitlinien der Deutschen Gesellschaft für Neurologie; 4. Auflage; Georg Thieme Verlag, Stuttgart 2008

Ohayon MM, Caulet M, Priest RG (1997): Violent behavior during sleep. J Clin Psychiatry $\underline{58}, 369-376$

Olanow CW, Tatton WG (1999): Etiology and pathogenesis of Parkinson's disease. Annu Rev Neurosci 22, 123-144

Olson EJ, Boeve BF, Silber MH (2000): Rapid eye movement sleep behaviour disorder: demographic, clinical and laboratory findings in 93 cases. Brain 123, 331-339

O'Sullivan SS, Williams DR, Gallagher DA, Massey LA, Silveira-Moriyama L, Lees AJ (2008): Nonmotor symptoms as presenting complaints in Parkinson's disease: A clinicopathological study. Mov Disord 23, 101-106

Otto M, Esselmann H, Schulz-Shaeffer W, Neumann M, Schröter A, Ratzka P, Cepek L, Zerr I, Steinacker P, Windl O et al. (2000): Decreased beta-amyloid1-42 in cerebrospinal fluid of patients with Creutzfeldt-Jakob disease. Neurology 54, 1099-1102.

Papapetropoulos S, Paschalis C, Athanassiadou A, Papadimitriou A, Ellul J, Polymeropoulos M, Papapetropoulos T (2001): Clinical phenotype in patients with $\alpha$ synuclein Parkinson's disease living in Greece in comparison with patients with sporadic Parkinson's disease. J Neurol Neurosurg Psychiatry $\underline{70}, 662-665$

Parkinson J (1817/ 2002): An Essay on the Shaking Palsy. J Neuropsychiatry Clin Neurosci 14 , 223-236 (im Original veröffentlicht von Sherwood, Neely und Jones, London 1817)

Parkkinen L, Kauppinen T, Pirttilä T, Autere JM, Alafuzoff I (2005): $\alpha$-Synuclein pathology does not predict extrapyramidal symptoms or dementia. Ann Neurol $\underline{57}, 82-91$

Parnetti L, Tiraboschi P, Lanari A, Peducci M, Padiglioni C, D'Amore C, Pierguidi L, Tambasco N, Rossi A, Calabresi P (2008): Cerebrospinal fluid biomarkers in Parkinson's disease with dementia and dementia with Lewy bodies. Biol Psychiatry $\underline{64}, 850-855$

Payami H, Larsen K, Bernard S, Nutt J (1994): Increased risk of Parkinson's disease in parents and siblings of patients. Ann Neurol 36, 659-661

Peskind ER, Riekse R, Quinn JF, Kaye J, Clark CM, Farlow MR, Decarli C, Chabal C, Vavrek D, Raskind MA et al. (2005): Safety and acceptability of the research lumbar puncture. Alzheimer Dis Assoc Disord 19, 220-225

Peto V, Jenkinson C, Fitzpatrick R, Greenhall R (1995): The development and validation of a short measure of functioning and well being for individuals with Parkinson's disease. Qual Life Res $\underline{4}$, 241-248

Peto V, Jenkinson C, Fitzpatrick R (1998): PDQ-39: a review of the development, validation and application of a Parkinson's disease quality of life questionnaire and its associated measures. J Neurol 245 (Suppl. 1), S10-S14

Ponsen MM, Stoffers D, Booij J, van Eck-Smit BLF, Wolters EC, Berendse HW (2004): Idiopathic hyposmia as a preclinical sign of Parkinson's disease. Ann Neurol $\underline{56}, 173-$ 181

Ponsen MM, Daffertshofer A, Wolters EC, Beek PJ, Berendse HW (2008): Impairment of complex upper limb motor function in de novo Parkinson's disease. Parkinsonism Relat Disord 14, 199-204 
Postuma RB, Gagnon JF, Vendette M, Fantini ML, Massicotte-Marquez J, Montplaisir J (2009 a): Quantifying the risk of neurodegenerative disease in idiopathic REM sleep behavior disorder. Neurology $\underline{72}, 1296-1300$

Postuma RB, Gagnon JF, Vendette M, Montplaisir JY (2009 b): Markers of neurodegeneration in idiopathic rapid eye movement sleep behaviour disorder and Parkinson's disease. Brain 132, 3298-3307

Price MJ, Feldman RG, Adelberg D, Kayne H (1992): Abnormalities in color vision and contrast sensitivity in Parkinson's disease. Neurology 42, 887-890.

Rahman S, Griffin HJ, Quinn NP, Jahanshahi M (2008): Quality of life in Parkinson's disease: The relative importance of the symptoms. Mov Disord 23, 1428-1434

Rajput AH, Rozdilsky B, Ang L (1991 a): Occurrence of resting tremor in Parkinson's disease. Neurology 41, 1298-1299

Rajput AH, Rozdilsky B, Rajput A (1991 b): Accuracy of clinical diagnosis in parkinsonism--a prospective study. Can J Neurol Sci 18, 275-278

Ravina B, Eidelberg D, Ahlskog JE, Albin RL, Brooks DJ, Carbon M, Dhawan V, Feigin A, Fahn S, Guttman M et al. (2005): The role of radiotracer imaging in Parkinson disease. Neurology $\underline{64}, 208-215$

Ravina B, Elm J, Camicioli R, Como PG, Marsh L, Jankovic J, Weintraub D (2009): The course of depressive symptoms in early Parkinson's disease. Mov Disord 24, 13061311

Reichmann H (2010): Zukünftige Diagnosestandards beim Parkinson-Syndrom. Fortschr Neurol Psychiatr 78 (Suppl. 1), S5-S7

Riedel O, Klotsche J, Spottke A, Deuschl G, Förstl H, Henn F, Heuser I, Oertel W, Reichmann $\mathrm{H}$, Riederer $\mathrm{P}$ et al. (2008): Cognitive impairment in 873 patients with idiopathic Parkinson's disease. Results from the German Study on Epidemiology of Parkinson's Disease with Dementia (GEPAD). J Neurol 255, 255-264

Riederer P, Jellinger K: Morphologie und Pathobiochemie der Parkinson-Krankheit; in: Pathophysiologie, Klinik und Therapie des Parkinsonismus; hrsg. v. Gänshirt H unter Mitarbeit von Berlit P und Haack G; Editiones Roche, Basel 1983, 31-50

Ritz B, Ascherio A, Checkoway H, Marder KS, Nelson LM, Rocca WA, Ross GW, Strickland D, Van Den Eeden SK, Gorell J (2007): Pooled analysis of tobacco use and risk of Parkinson disease." Arch Neurol 64, 990-997

Robertson D (2008): The pathophysiology and diagnosis of orthostatic hypotension. Clin Auton Res 18 (Suppl. 1), 2-7

Rutan GH, Hermanson B, Bild DE, Kittner SJ, LaBaw F, Tell GS (1992): Orthostatic hypotension in older adults. The Cardiovascular Health Study. CHS Collaborative Research Group. Hypertension 19, 508-519

Sachs C, Berglund B, Kaijser L (1985): Autonomic cardiovascular responses in parkinsonism: effect of levodopa with dopa-decarboxylase inhibition. Acta Neurol Scand $\underline{71}, 37-42$

Sakakibara R, Uchiyama T, Yamanishi T, Kishi M (2010): Genitourinary dysfunction in Parkinson's disease. Mov Disord 25, 2-12

Schenck CH, Bundlie SR, Mahowald MW (1996): Delayed emergence of a parkinsonian disorder in $38 \%$ of 29 older men initially diagnosed with idiopathic rapid eye movement sleep behaviour disorder. Neurology $\underline{46}, 388-393$

Scherzer CR, Eklund AC, Morse LJ, Liao Z, Locascio JJ, Fefer D, Schwarzschild MA, Schlossmacher MG, Hauser MA, Vance JM et al. (2007): Molecular markers of early Parkinson's disease based on gene expression in blood. Proc Natl Acad Sci U S A $\underline{104}, 955-960$

Schrag A, Jahanshahi M, Quinn N (2000): How does Parkinson's disease affect quality of life? A comparison with quality of life in the general population. Mov Disord $\underline{15}, 1112-$ 1118 
Schrag A, Ben-Shlomo Y, Quinn N (2002 a): How common are complications of Parkinson's disease? J Neurol 249, 419-423

Schrag A, Ben-Shlomo Y, Quinn N (2002 b): How valid is the clinical diagnosis of Parkinson's disease in the community? J Neurol Neurosurg Psychiatry $\underline{73}, 529-534$

Schulz-Schaeffer WJ (2010): The synaptic pathology of a-synuclein aggregation in dementia with Lewy bodies, Parkinson's disease and Parkinson's disease dementia. Acta Neuropathol 120, 131-143.

Schwarzschild MA, Schwid SR, Marek K, Watts A, Lang AE, Oakes D, Shoulson I, Ascherio A, the Parkinson Study Group PRECEPT Investigators (2008): Serum Urate as a Predictor of Clinical and Radiographic Progression in Parkinson Disease. Arch Neurol $\underline{65}, 716-723$

Schweitzer KJ, Hilker R, Walter U, Burghaus L, Berg D (2006): Substantia nigra hyperechogenicity as a marker of predisposition and slower progression in Parkinson's disease. Mov Disord 21, 94-98

Shulman KI, Shedletsky R, Silver IL (1986): The challenge of time: Clock-drawing and cognitive function in the elderly. Int J Geriatr Psychiatry $\underline{1}$, 135-140

Shulman KI, Gold DP, Cohen CA, Zucchero CA (1993): Clock-drawing and dementia in the community: A longitudinal study. Int J Geriatr Psychiatry $\underline{8}$, 487-496

Shulman LM, Taback RL, Rabinstein AA, Weiner WJ (2002): Non-recognition of depression and other non-motor symptoms in Parkinson's disease. Parkinsonism Relat Disord $\underline{8}$, 193-197

Siderowf A, Xie SX, Hurtig H, Weintraub D, Duda J, Chen-Plotkin A, Shaw LM, Van Deerlin V, Trojanowski JQ, Clark C (2010): CSF amyloid B 1-42 predicts cognitive decline in Parkinson disease. Neurology $\underline{75}, 1055-1061$

Sixel-Doring F, Schweitzer M, Mollenhauer B, Trenkwalder C (2009): REM sleep behavior disorder severity scale: A video classification of RBD in Parkinson's disease. Mov Disord 24 (Suppl. 1), S395-S396

Sjögren M, Minthon L, Davidsson P, Granérus A-K, Clarberg A, Vanderstichele H, Vanmechelen E, Wallin A, Blennow K (2000): CSF levels of tau, $\beta$-amyloid(1-42) and GAP-43 in frontotemporal dementia, other types of dementia and normal aging. J Neural Transm 107, 563-579

Snyder SW, Ladror US, Wade WS, Wang GT, Barrett LW, Matayoshi ED, Huffaker HJ, Krafft GA, Holzman TF (1994): Amyloid-beta aggregation: selective inhibition of aggregation in mixtures of amyloid with different chain lengths. Biophys $\mathrm{J} \underline{67}, 1216-1228$

Sommer U, Hummel T, Cormann K, Mueller A, Frasnelli J, Kropp J, Reichmann H (2004): Detection of presymptomatic Parkinson's disease: combining smell tests, transcranial sonography, and SPECT. Mov Disord 19, 1196-1202

Spillantini MG, Schmidt ML, Lee VM, Trojanowski JQ, Jakes R, Goedert M (1997): Asynuclein in Lewy bodies. Nature $\underline{388}$, 839-840

Statistisches Bundesamt Deutschland (Stand: 2010): Bevölkerung nach Altersgruppen, Familienstand und Religionszugehörigkeit. URL: https://www.destatis.de/DE/ ZahlenFakten/GesellschaftStaat/Bevoelkerung/Bevoelkerungsstand/Tabellen/Altersgr uppenFamilienstand.html?nn=50732 [letzter Abruf: 19.03.2012]

Statistisches Bundesamt Deutschland (Stand: 31.12.2010): Bevölkerung. URL: https://www.destatis.de/E/ZahlenFakten/GesellschaftStaat/Bevoelkerung/Bevoelkerun gsstand/Aktuell.html?nn=50732 [letzter Abruf: 19.03.2012]

Stiasny-Kolster K, Doerr Y, Möller JC, Höffken H, Behr TM, Oertel WH, Mayer G (2005): Combination of 'idiopathic' REM sleep behaviour disorder and olfactory dysfunction as possible indicator for alpha-synucleinopathy demonstrated by dopamine transporter FP-CIT-SPECT. Brain 128, 126-137

Stiasny-Kolster K, Mayer G, Schäfer S, Möller JC, Heinzel-Gutenbrunner M, Oertel WH (2007): The REM sleep behavior disorder screening questionnaire - A new diagnostic instrument. Mov Disord 22, 2386-2393 
Stocchi F, Carbone A, Inghilleri M, Monge A, Ruggieri S, Berardelli A, Manfredi M (1997): Urodynamic and neurophysiological evaluation in Parkinson's disease and multiple system atrophy. J Neurol Neurosurg Psychiatry $\underline{62}, 507-511$

Storch A, Odin P, Trender-Gerhard I, Fuchs G, Reifschneider G, Ray Chaudhuri K, Jost W, Ebersbach G (2010): Non-motor Symptoms Questionnaire und Scale für das idiopathische Parkinson-Syndrom. Nervenarzt 81, 980-985

Strozyk D, Blennow K, White LR, Launer LJ (2003): CSF Abeta 42 levels correlate with amyloid-neuropathology in a population-based autopsy study. Neurology $\underline{60}, 652-656$

Takahashi H, Ohama E, Suzuki S, Horikawa Y, Ishikawa A, Morita T, Tsuji S, Ikuta F (1994): Familial juvenile parkinsonism: clinical and pathologic study in a family. Neurology $\underline{44}$, 437-441

Tandberg E, Larsen JP, Karlsen K (1998): A community-based study of sleep disorders in patients with Parkinson's disease. Mov Disord 13, 895-899

Tang MX, Stern Y, Marder K, Bell K, Gurland B, Lantigua R, Andrews H, Feng L, Tycko B, Mayeux R (1998): The APOE-ع4 Allele and the Risk of Alzheimer Disease Among African Americans, Whites, and Hispanics." JAMA 279, 751-755

Tanner CM, Ottman R, Goldman SM, Ellenberg J, Chan P, Mayeux R, Langston JW (1999): Parkinson disease in twins: an etiologic study. JAMA 281, 341-346

Taylor CA, Saint-Hilaire MH, Cupples LA, Thomas CA, Burchard AE, Feldman RG, Myers $\mathrm{RH}$ (1999): Environmental, medical, and family history risk factors for Parkinson's disease: a New England-based case control study. Am J Med Genet 88, 742-749

Taylor KSM, Cook JA, Counsell CE (2007): Heterogeneity in male to female risk for Parkinson's disease. J Neurol Neurosurg Psychiatry 78, 905-906

Tetrud JW (1991): Preclinical Parkinson's disease: detection of motor and nonmotor manifestations. Neurology 41 (Suppl. 2), 69-72

The Consensus Committee of the American Autonomic Society and the American Academy of Neurology (1996): Consensus statement on the definition of orthostatic hypotension, pure autonomic failure, and multiple system atrophy. Neurology $\underline{46}$, 1470

The Ronald and Nancy Reagan Research Institute of the Alzheimer's Association and National Institute on Aging Working Group (1998): Consensus report of the Working Group on: "Molecular and Biochemical Markers of Alzheimer's Disease". Neurobiol Aging 19, 109-116

Tiedt N (1985): Das Orthostasesyndrom - pathologische, funktionsdiagnostische und physiotherapeutische Aspekte. Teil I: Pathophysiologie und diagnostische Prinzipien. Z Physiother 37, 145-166

Tissingh G, Berendse $\mathrm{H}$, Bergmans $\mathrm{P}$, DeWaard R, Drukarch B, Stoof J, Wolters E (2001): Loss of olfaction in de novo and treated Parkinson's disease: Possible implications for early diagnosis. Mov Disord $\underline{16}, 41-46$

Todes CJ, Lees AJ (1985): The pre-morbid personality of patients with Parkinson's disease. $\mathrm{J}$ Neurol Neurosurg Psychiatry $\underline{48}, 97-100$

Tokuda T, Salem SA, Allsop D, Mizuno T, Nakagawa M, Qureshi MM, Locascio JJ, Schlossmacher MG, El-Agnaf OMA (2006): Decreased alpha-synuclein in cerebrospinal fluid of aged individuals and subjects with Parkinson's disease. Biochem Biophys Res Commun 349, 162-166

Tolosa E, Wenning G, Poewe W (2006): The diagnosis of Parkinson's disease. Lancet Neurol $\underline{5}, 75-86$.

Tolosa E, Gaig C, Santamaria J, Compta Y (2009): Diagnosis and the premotor phase of Parkinson disease. Neurology 72 (Suppl. 2), S12-S20

Toth C, Rajput M, Rajput AH (2004): Anomalies of asymmetry of clinical signs in parkinsonism. Mov Disord 19, 151-157 
Trenkwalder C, Schwarz J, Gebhard J, Ruland D, Trenkwalder P, Hense HW, Oertel WH (1995): Starnberg trial on epidemiology of Parkinsonism and hypertension in the elderly. Prevalence of Parkinson's disease and related disorders assessed by a doorto-door survey of inhabitants older than 65 years. Arch Neurol 52, 1017-1022

Tsuboi Y, Yamada T, Chaudhuri RK, Martinez-Martin P, Schapira AH, The International P (2006): Comparison of profile of non-motor symptoms in Japanese patients with PD with European patients and healthy controls. Extension of the NMSQuest study. Mov Disord 21 (Suppl. 15), S648

Uitti RJ, Baba Y, Whaley NR, Wszolek ZK, Putzke JD (2005): Parkinson disease: handedness predicts asymmetry. Neurology 64, 1925-1930

Valls-Solé J, Valldeoriola F (2002): Neurophysiological correlate of clinical signs in Parkinson's disease. Clin Neurophysiol 113, 792-805.

Van Den Eeden SK, Tanner CM, Bernstein AL, Fross RD, Leimpeter A, Bloch DA, Nelson LM (2003): Incidence of Parkinson's Disease: Variation by Age, Gender, and Race/Ethnicity. Am J Epidemiol 157, 1015-1022

von Campenhausen S, Bornschein B, Wick R, Bötzel K, Sampaio C, Poewe W, Oertel W, Siebert U, Berger K, Dodel R (2005): Prevalence and incidence of Parkinson's disease in Europe. Eur Neuropsychopharmacol 15, 473-490

von Reichmann H, Deuschl G, Riedel O, Spottke A, Förstl H, Henn F, Heuser I, Oertel W, Riederer P, Trenkwalder C et al. (2010): Die "German Study on the Epidemiologyof Parkinson's Disease with Dementia (GEPAD)": Mehr als nur Parkinson. MMW Fortschr Med 152 (Suppl. 1), 1-6

Wakabayashi K, Takahashi H, Takeda S, Ohama E, Ikuta F (1988): Parkinson's disease: the presence of Lewy bodies in Auerbach's and Meissner's plexuses. Acta Neuropathol 76, 217-221.

Wakabayashi K, Tanji K, Mori F, Takahashi H (2007): The Lewy body in Parkinson's disease: Molecules implicated in the formation and degradation of $\alpha$-synuclein aggregates. Neuropathology 27, 494-506

Walter U, Wittstock M, Benecke R, Dressler D (2002): Substantia nigra echogenicity is normal in non-extrapyramidal cerebral disorders but increased in Parkinson's disease. J Neural Transm 109, 191-196

Walter U, Niehaus L, Probst T, Benecke R, Meyer BU, Dressler D (2003): Brain parenchyma sonography discriminates Parkinson's disease and atypical parkinsonian syndromes. Neurology $\underline{60}, 74-77$

Walter U, Dressler D, Benecke R (2004): Hirnparenchym-Sonographie zur Früh- und Differenzialdiagnostik der Parkinson-Krankheit. Akt Neurol 31, 325-332

Webster Ross G, Petrovitch H, Abbott RD, Tanner CM, Popper J, Masaki K, Launer L, White LR (2008): Association of olfactory dysfunction with risk for future Parkinson's disease. Ann Neurol 63, 167-173

Weintraub D, Moberg PJ, Duda JE, Katz IR, Stern MB (2004): Effect of Psychiatric and Other Nonmotor Symptoms on Disability in Parkinson's Disease. J Am Geriatr Soc 52, 784-788

Wenning GK, Shephard B, Hawkes C, Petruckevitch A, Lees A, Quinn N (1995): Olfactory function in atypical parkinsonian syndromes. Acta Neurol Scand 91, 247-250

Wenning GK, Stefanova N, Jellinger KA, Poewe W, Schlossmacher MG (2008): Multiple system atrophy: a primary oligodendrogliopathy. Ann Neurol 64, 239-246

Wetter TC, Collado-Seidel V, Trenkwalder C (1999): Polysomnographische Untersuchungen von Patienten mit Parkinson-Syndrome im Vergleich zu gesunden Kontrollen. Somnologie $\underline{3}, 300-306$

Wetter TC, Collado-Seidel V, Pollmächer T, Yassouridis A, Trenkwalder C (2000): Sleep and periodic leg movement patterns in drug-free patients with Parkinson's disease and multiple system atrophy. Sleep $\underline{23}, 361-367$ 
Wider C, Ross OA, Wszolek ZK (2010): Genetics of Parkinson disease and essential tremor. Curr Opin Neurol 23, 388-393

Williams DR, Lees AJ (2009): How do patients with parkinsonism present? A clinicopathological study. Intern Med J 39, 7-12.

Williams-Gray CH, Foltynie T, Brayne CEG, Robbins TW, Barker RA (2007): Evolution of cognitive dysfunction in an incident Parkinson's disease cohort. Brain 130, 1787-1798

Winge K, Werdelin LM, Nielsen KK, Stimpel H (2004): Effects of dopaminergic treatment on bladder function in Parkinson's disease. Neurourol Urodyn 23, 689-696

Woitalla D, Braak H, Tredici KD, Fogel W, Hagenah J, Oertel W, Berg D (2010): Stellenwert der Hirnparenchym-Sonografie in der Differenzial- und Frühdiagnose des ParkinsonSyndroms. Fortschr Neurol Psychiat 78 (Suppl. 1), S25-S30

Ziegler U, Doblhammer G (2009): Prävalenz und Inzidenz von Demenz in Deutschland: eine Studie auf Basis von Daten der gesetzlichen Krankenversicherungen von 2002. Gesundheitswesen 71, 281-290

Zorzon M, Capus L, Pellegrino A, Cazzato G, Zivadinov R (2002): Familial and environmental risk factors in Parkinson's disease: a case-control study in north-east Italy. Acta Neurol Scand 105, 77-82 


\section{LEBENSLAUF}

Am 16. August 1986 wurde ich in Leverkusen geboren. Meine Eltern sind Ursula Werner, geb. Pötz, und Walter Werner.

Von 1992 bis 1994 besuchte ich die Grundschule am Jacobitor in Osterode/ Harz und von 1994 bis 1996 die Zastrowschule, evangelische Grundschule, in Mühlheim/ Ruhr. Anschließend wechselte ich zur Karl-Ziegler-Schule, städtisches Gymnasium, in Mühlheim/ Ruhr. Von 1998 bis 2005 besuchte ich das Goethe-Gymnasium in Kassel, an dem ich im Juni 2005 die allgemeine Hochschulreife erwarb. Von September bis Dezember 2005 absolvierte ich Praktika in der Käthe-Kollwitz-Schule, Schule für Praktisch Bildbare, in Hofgeismar und in der Montessori-Schule in Kassel. Von Januar bis März 2006 arbeitete ich im Krankenpflegedienst im Kinderkrankenhaus Park Schönfeld. Im April 2006 machte ich einen sozial-diakonischen Einsatz in Nairobi, Kenia.

Seit dem Wintersemester 2006/07 studiere ich Humanmedizin an der Georg-AugustUniversität Göttingen. Am 11. September 2008 legte ich den ersten Abschnitt der ärztlichen Prüfung ab. Seit Juli 2009 bin ich Doktorandin in der Paracelsus-Elena-Klinik Kassel bei Prof. Dr. med. B. Mollenhauer. 